\title{
Evaluation of a modern implementation of dynamic conformal arc therapy
}

by

\author{
Lorcel Ericka T. Venturina
}

A thesis submitted to the Faculty of Graduate and Postdoctoral Affairs in partial fulfillment of the requirements for the degree of

\author{
Master of Science
}

in

Physics

Specialization in Medical Physics

Ottawa-Carleton Institute for Physics

Department of Physics

Carleton University

Ottawa, Ontario, Canada

November 2017

(C) 2017 Lorcel Ericka T. Venturina 
THIS PAGE IS INTENTIONALLY LEFT BLANK 


\section{Abstract}

Dynamic Conformal Arc Therapy (DCAT) is an external beam radiation therapy technique in which the cancer target is treated with the gantry head of the medical linear accelerator rotating around the patient while conforming the radiation beam to the target shape at each gantry angle. A modern implementation of DCAT has recently been released, in which the dose rate can be varied and the beam can be modulated moderately. In this study, this modern implementation of DCAT (mDCAT) was characterized, and its potential advantages over the most commonly used treatment techniques, namely 3D Conformal Radiation Therapy (3DCRT) and Volumetric Modulated Arc Therapy (VMAT), were evaluated in terms of efficiency, quality, and robustness. Results show that plan optimization with mDCAT is up to three times more efficient than VMAT. mDCAT plan quality is midway between 3DCRT and VMAT for large targets, and is very comparable to VMAT for small targets. mDCAT and VMAT are equally robust against typical machine and patient uncertainties. 


\section{Acknowledgements}

Firstly, I would like to express my deepest gratitude to my supervisor, Dr. Elsayed Ali, for his guidance, patience and support in both my research and academic undertakings. Coming back to academia after several years of working was a big transition for me, and Dr. Ali was there to assist and guide me, and for that I am grateful.

I thank Greg Fox, an experienced treatment planner from The Ottawa Hospital Cancer Centre, for his invaluable contribution to the treatment planning part of this study, making sure that the plans meet high quality clinical standards.

I greatly appreciate the help of Dr. Jason Belec, Dr. Justin Sutherland, Dr. Dal Granville and Cathy Rose of The Ottawa Hospital Cancer Centre.

I'm grateful to the generous support from the following scholarship funding: Queen Elizabeth II Graduate Scholarship in Science and Technology, the Medical Physics Department at The Ottawa Hospital, and the Physics Department at Carleton University.

To my fiancé and best friend, Marvin: your presence is the nicest part of these past two years. Thank you for your patience. To my parents and siblings, you are my inspiration in pursuing my dreams. Thank you for your encouragement, love and support.

Dedication: This thesis is dedicated to my mother, Lorcelie, my father, Enrico, and my grandmother, Leticia. 


\section{Ethics and originality statement}

This thesis is the summary of the most significant portion of the author's work during the course of her M.Sc. program at Carleton University.

This study is part of a Quality Improvement Initiative at The Ottawa Hospital Cancer Centre, and is exempt from a full ethics review. Patient data included in this study were anonymized, and The Ottawa Hospital confidentiality and ethics rules were followed. The Carleton University Research Ethics office deferred to the assessment made at The Ottawa Hospital (Mr. Gordon DuVal, Director).

Dr. Elsayed Ali supervised the project and provided input on all of its components, including the publications. Greg Fox reviewed the treatment plans and modified them if necessary, making sure that the treatment plans were meeting clinical standards. He also created treatment plans from scratch for those patients without base plans. Otherwise, the author of this thesis performed all the computational works, performed the delivery of the radiation treatment plans of the different treatment modalities on the treatment units, and drafted and revised all the manuscripts. All figures, graphs and tables presented in this thesis are original production of the author. 


\section{Publications}

\section{Peer-reviewed papers}

1. L. E. T. Venturina and E. S. M. Ali, "Dynamic conformal arc therapy with variable dose rate and segment shape optimization", Phys. Med. Biol., submitted, Jan. 2018.

2. L. E. T. Venturina, G. Fox and E. S. M. Ali, "Dosimetric evaluation of dynamic conformal arc therapy with variable dose rate and moderate modulation in comparison to three-dimensional conformal radiotherapy and volumetric modulated arc therapy for spine, lung and brain tumors", Med. Dosim., in preparation (2018).

\section{Conference abstracts}

L. E. T. Venturina, G. Fox and E. S. M. Ali, "Characterization of a modern implementation of dynamic conformal arc therapy", $63^{\text {rd }}$ COMP Annual Scientific Meeting, Ottawa, Ontario, Canada, Jul. 2017, oral presentation. Published in Med. Phys. 44 4395 (2017) 


\section{Contents}

$\begin{array}{lll}\text { Abstract } & \text { i }\end{array}$

$\begin{array}{ll}\text { Acknowledgements } & \text { ii }\end{array}$

Ethics and originality statement $\quad$ iii

Publications $\quad$ iv

Acronyms $\quad$ viii

List of Tables $\quad$ xi

List of Figures $\quad$ xii

1 Introduction $\quad 1$

1.1 The External Beam Radiation Therapy (EBRT) process . . . . . . . . . 2

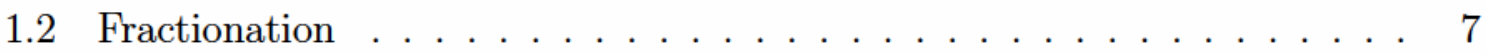

1.3 CT simulation of lung patients ............... . 8

1.4 LINAC in photon mode . . . . . . . . . . . . . . . . . . . . . 11

1.5 Treatment techniques in photon mode . . . . . . . . . . . . . 13

1.6 Motivation and contributions .................. 20

1.7 Review of previous studies on classical DCAT (cDCAT) . . . . . . . . 21

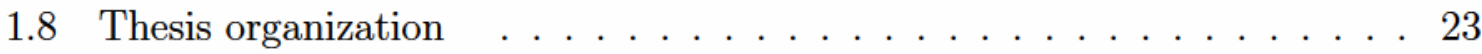


2 Treatment planning $\quad 24$

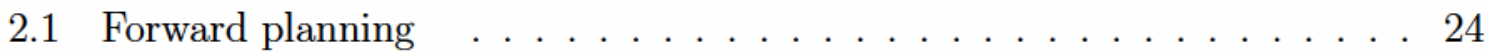

2.2 Inverse planning . . . . . . . . . . . . . . . . . . 25

2.3 Radiation beam model . . . . . . . . . . . . . . . . . 35

2.4 Dose calculation algorithms . . . . . . . . . . . . . . . . 35

2.5 Patient selection $\ldots \ldots \ldots \ldots$. . . . . . . . . . . . . . . . . 39

2.6 Treatment planning methodologies . . . . . . . . . . . . . . . 44

2.7 Plan quality assessment metrics . . . . . . . . . . . . . . . . . 47

3 Characterization of modern DCAT (mDCAT) 53

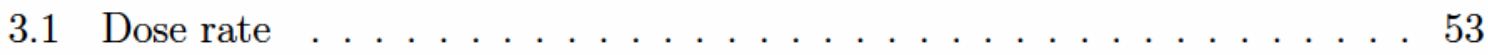

3.2 Target-to-MLC margin . . . . . . . . . . . . . . . 57

3.3 Arc increment . . . . . . . . . . . . . . . . . . . . 59

3.4 Avoidance structure . . . . . . . . . . . . . . . . . . . . 60

3.5 Segment Shape Optimization $(\mathrm{SSO}) \ldots \ldots \ldots$. . . . . . . . . . 60

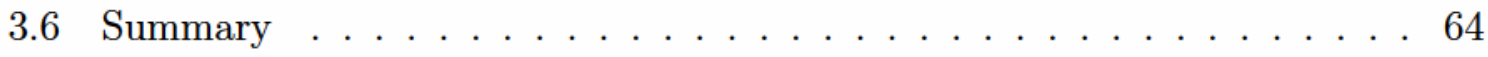

4 Efficiency of plan optimization and treatment delivery 65

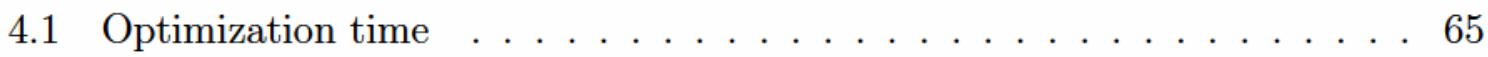

4.2 Treatment delivery time .................. 70

4.3 Monitor units and modulation factor . . . . . . . . . . . . . 71

4.4 Summary .............................. 71

$5 \quad$ Plan quality $\quad 73$

5.1 Plan quality for spine ..................... 73

5.2 Plan quality for lung SBRT . . . . . . . . . . . . . . . 79 
5.3 Plan quality for brain $\ldots \ldots \ldots \ldots \ldots$

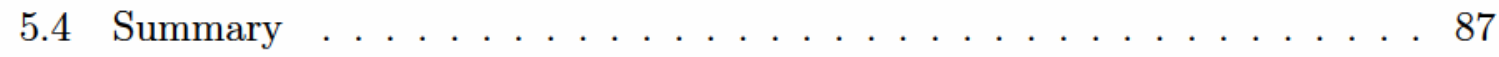

6 Robustness against machine uncertainties $\quad 88$

6.1 Modelling machine errors . . . . . . . . . . . . . . . . . . . . . . . 89

6.2 Dosimetric impact of extreme MLC errors . . . . . . . . . . . . . . 92

6.3 Dosimetric impact of typical MLC errors . . . . . . . . . . . . . . . 101

6.4 Summary . . . . . . . . . . . . . . . . . . 106

$\begin{array}{lll}7 & \text { Robustness against patient uncertainties } & 107\end{array}$

7.1 Modelling tumour motion due to breathing . . . . . . . . . 107

7.2 Modelling tumour motion using isocentre-moves only . . . . . . . . . 112

7.3 Dosimetric impact: isocentre-moves only . . . . . . . . . . . . . . . 112

7.4 Modelling interplay effect $\ldots \ldots \ldots \ldots \ldots$

7.5 Dosimetric impact: interplay effect . . . . . . . . . . . . . . . 123

7.6 Combining interplay effect with setup errors $\ldots \ldots \ldots$. . . . . . 133

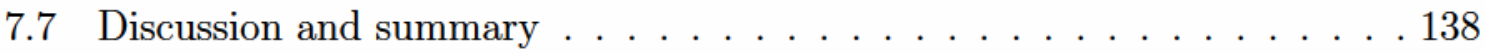

8 Summary, conclusions, and future work 141

Appendix: Anonymized list of patients 145

$\begin{array}{ll}\text { References } & 154\end{array}$ 


\section{Acronyms}

$\begin{array}{ll}\text { 3DCRT } & \text { Three-Dimensional Conformal Radiation Therapy } \\ \text { 4DCT } & \text { Four-Dimensional Computed Tomography } \\ \text { BEV } & \text { Beam's Eye View } \\ \text { CBCT } & \text { Cone-Beam Computed Tomography } \\ \text { cDCAT } & \text { classical Dynamic Conformal Arc Therapy } \\ \text { cGy } & \text { centiGray } \\ \text { CI } & \text { Conformity Index } \\ \text { CT } & \text { Computed Tomography } \\ \text { CTV } & \text { Clinical Target Volume } \\ \text { D } \% & \text { Dose received by } x \% \text { of the volume of a structure (units: cGy) } \\ \text { DRR } & \text { Digitally Reconstructed Radiograph } \\ \text { DVH } & \text { Dose Volume Histogram } \\ \text { EBRT } & \text { External Beam Radiation Therapy } \\ \text { ED } & \text { Electron Density } \\ \text { EUD } & \text { Equivalent Uniform Dose } \\ \text { GI } & \text { Gradient Index } \\ \text { GTV } & \text { Gross Tumor Volume } \\ \text { Gy } & \text { Gray }\end{array}$


HI

HU

ICRU

IM

IMRT

IQR

ITV

LCS

LINAC

mDCAT

MIP

MLC

MU

MV

OAR

PTV

QA

QUANTEC

RT

RTOG

$\mathrm{Rx}$

SABR
Homogeneity Index

Hounsfield Unit

International Commission on Radiation Units and Measurements

Internal Margin

Intensity Modulated Radiation Therapy

Inter-Quartile Range

Internal Target Volume

LINAC Control System

LINear ACcelerator

modern Dynamic Conformal Arc Therapy

Maximum Intensity Projection

Multi-Leaf Collimator

Monitor Unit

MegaVoltage

Organ At Risk

Planning Target Volume

Quality Assurance

Quantitative Analyses of Normal Tissue Effects in the Clinic

Radiation Therapy

Radiation Therapy Oncology Group

Prescription dose

Stereotactic ABlative Radiotherapy 
SBRT

SM

SRS

SSO

TOHCC

TPS

$\mathrm{V}_{x G y}$

VMAT
Stereotactic Body Radiation Therapy

Setup Margin

Stereotactic RadioSurgery

Segment Shape Optimization

The Ottawa Hospital Cancer Centre

Treatment Planning System

Fractional volume of a structure receiving x Gy (units: \%)

Volumetric Modulated Arc Therapy 


\section{List of Tables}

2.1 Dose differences with different calculation algorithms for spine . . . . . . 39

2.2 Dose differences with different calculation algorithms for lung SBRT . . . 39

2.3 Breakdown of spine patients by target location . . . . . . . . . . . . 42

2.4 OAR dose limits and toxicities . . . . . . . . . . . . . . . . 52

4.1 Optimization times for mDCAT and VMAT for spine . . . . . . . . 68

4.2 Optimization times for mDCAT and VMAT using $5 \mathrm{~mm}$ calculation grid 68

4.3 Optimization times for mDCAT and VMAT for lung SBRT . . . . . . . . 69

4.4 Optimization times for mDCAT and VMAT for brain . . . . . . . . . . 69

4.5 Correlation between target size and normalized optimization times . . . . 70

4.6 Treatment time for mDCAT and VMAT . . . . . . . . . . . . 70

4.7 Modulation factor for mDCAT and VMAT . . . . . . . . . . . . . 71

5.1 Plan quality metrics for spine . . . . . . . . . . . . . . 77

5.2 Plan quality metrics for lung SBRT . . . . . . . . . . . . . . . . 80

5.3 Plan quality metrics for brain . . . . . . . . . . . . . . . 84

6.1 Impact on plan quality: extreme systematic MLC leaf errors . . . . . . . 97

6.2 Impact on plan quality: typical systematic MLC leaf errors . . . . . . . . 102

7.1 Impact on plan quality: tumour motion with only isocentre moves . . . . 114

7.2 Impact on plan quality: combined tumour motion and setup error . . . . 128 


\section{List of Figures}

1.1 An Elekta Synergy linear accelerator . . . . . . . . . . . . . . . . 2

1.2 Different CT scan views . . . . . . . . . . . . . . . . . . . . 3

1.3 Digitally Reconstructed Radiographs . . . . . . . . . . . . . . . . . 4

1.4 A schematic of target volumes .................. . . 5

1.5 Examples of immobilization devices . . . . . . . . . . . . . . . . 9

1.6 The Elekta Agility collimation system assembly . . . . . . . . . . . . . . 13

1.7 Examples of 3DCRT beam arrangements . . . . . . . . . . . . 16

1.8 Photon beam fluence maps and beam segment shapes . . . . . . . . . . . 19

2.1 Input parameters for 3DCRT planning in the Monaco TPS . . . . . . . . 25

2.2 Dynamic parameter limits for Elekta LINACs with Agility collimation . . 28

2.3 Structure layering in the Monaco TPS . . . . . . . . . . . . . . 29

2.4 Physical cost functions . . . . . . . . . . . . . . . . . 30

2.5 Biological cost functions . . . . . . . . . . . . . . . . . 32

2.6 VMAT and mDCAT sequencing parameters . . . . . . . . . . . 34

2.7 Dose distribution in Monaco TPS . . . . . . . . . . . . . . . . . 48

2.8 Schematic of a DVH and dose-volume points . . . . . . . . . . . . . . 49

2.9 Potential conformity index limitations . . . . . . . . . . . . 50

3.1 mDCAT plans with constant versus variable dose rate . . . . . . . . . 55

3.2 mDCAT plans with constant versus variable dose rate for $\mathrm{D}_{95 \%}=\mathrm{Rx}$. . 56 
3.3 Plan quality for very tight versus wide target-to-MLC margin . . . . . . 58

3.4 SSO characterization . . . . . . . . . . . . . . . . 62

3.5 Beam's Eye View of mDCAT versus VMAT beam segments . . . . . . . 63

4.1 Plan optimization times . . . . . . . . . . . . . . 6 68

4.2 Correlation between target size and normalized optimization times . . . . 69

5.1 Plan quality for spine cases . . . . . . . . . . . . . . 76

5.2 OAR doses for spine cases . . . . . . . . . . . . . . 77

5.3 Dose distributions and DVHs for an example spine case . . . . . . . . 78

5.4 Plan quality for lung SBRT cases . . . . . . . . . . . . . . . 81

5.5 Dose distributions and DVHs for an example lung SBRT case . . . . . . 82

5.6 Plan quality for brain cases . . . . . . . . . . . . . . . . 85

5.7 Dose distributions and DVHs for an example brain case . . . . . . . . . 86

6.1 Error distribution of MLC leaf positions from QA data . . . . . . . . . 90

6.2 Error distribution of MLC leaf positions from plan deliveries on one LINAC 91

6.3 Actual delivery MLC errors per disease site . . . . . . . . . . . . . . . 92

6.4 Impact on plan quality for spine: extreme systematic MLC errors . . . . 98

6.5 Impact on plan quality for lung SBRT: extreme systematic MLC errors . 99

6.6 Impact on plan quality for brain: extreme systematic MLC errors . . . . 100

6.7 Impact on plan quality for spine: typical systematic MLC errors . . . . . 103

6.8 Impact on plan quality for lung SBRT: typical systematic MLC errors . . 104

6.9 Impact on plan quality for brain: typical systematic MLC errors . . . . . 105

7.1 Sinusoidal model for breathing . . . . . . . . . . . . . . . . 109

7.2 Impact on plan quality: tumour motion with only isocentre moves . . . . 115 
7.3 Screenshot of a plan file in the back end of the Monaco TPS . . . . . . . 117

7.4 Simulated versus measured dynamic parameters for mDCAT . . . . . . . 120

7.5 Simulated versus measured dynamic parameters for VMAT . . . . . . . . 121

7.6 Simulated versus measured delivery times for individual beam segments . 122

7.7 Schematic of an extreme case of the interplay effect . . . . . . . . . . 124

7.8 Impact on plan quality: tumour motion interplay with delivery . . . . . . 129

7.9 Dose difference maps for extreme tumour motion versus no motion . . . . 130

7.10 Interplay effect for extreme breathing amplitude . . . . . . . . . . . . 131

7.11 Interplay effect for typical breathing amplitude . . . . . . . . . . . . . 132

7.12 Impact on plan quality: combined tumour motion and setup error . . . . 135

7.13 Interplay effect combined with setup error for extreme breathing amplitude136

7.14 Interplay effect combined with setup error for typical breathing amplitude137 


\section{Chapter 1}

\section{Introduction}

Radiation Therapy (RT) is a form of cancer treatment in which high energy rays (such as photons) or particles (such as electrons and protons) are used to destroy cancer cells. ${ }^{1,2}$ The intent of treating with radiation is either to cure cancer (i.e., radical treatment) or to relieve symptoms such as pain and bleeding (i.e., palliative treatment). ${ }^{3}$ Radiation can be delivered either externally by delivering high energy radiation from a source located outside the body (known as External Beam Radiation Therapy, or EBRT) or internally by temporarily or permanently implanting radioactive materials in the tumour (known as brachytherapy). ${ }^{1,2}$ Radiation dose has a unit of Gray (Gy) which corresponds to the energy deposited by the radiation per unit mass of the irradiated tissues (Gray $=$ Joules per kilogram), and a centiGray (cGy) is equivalent to $1 / 100$ th of a Gray. ${ }^{1,2}$ The majority of patients undergoing RT are treated with EBRT. For superficial tumors, electron beams in the megavoltage energy range and photon beams in the kilovoltage energy range are mostly used. For large and/or deeply seated tumors, photon beams in the megavoltage (MV) energy range are mostly used. The focus of this study is on EBRT using photon beams in the MV energy range, which are generated using a medical LINear ACcelerator (LINAC). Shown in Figure 1.1 is the Elekta Synergy TM LINAC used in this study. A photon beam is generated by accelerating electrons through a wave guide inside the LINAC. The accelerated electrons are guided and focused towards a photon target which is typically made of a high atomic number material. Upon 


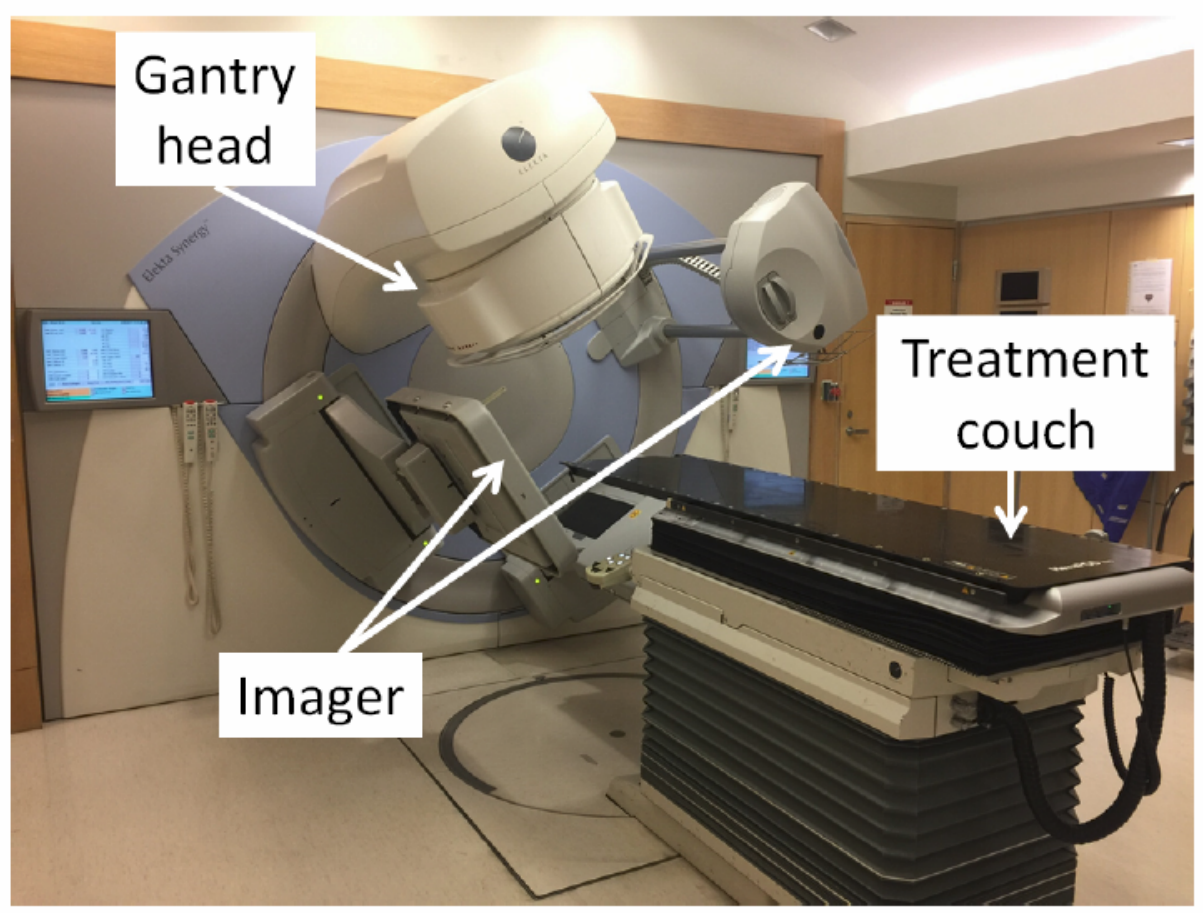

Figure 1.1: The Elekta Synergy medical LINAC used to deliver the radiation treatments in this study. A computational model of this LINAC was used for the treatment planning component of this study.

hitting the photon target, the electrons decelerate and produce photons with an energy spectrum from effectively zero to the maximum energy of the incident electrons. ${ }^{1,2}$ The photon beam is then shaped towards the area of treatment. The typical energy range of modern photon beams is 6 to $18 \mathrm{MV}$. Radiation treatment techniques presented in this study use photon beams, mostly $6 \mathrm{MV}$, which is the most commonly used photon beam energy in EBRT.

\subsection{The External Beam Radiation Therapy (EBRT) process}

The EBRT process consists of three main steps: patient simulation, treatment planning, and treatment delivery. ${ }^{4}$

The first step is patient simulation. Computed Tomography (CT) scans of the patient are acquired in the same position that the patient will be treated. These CT 


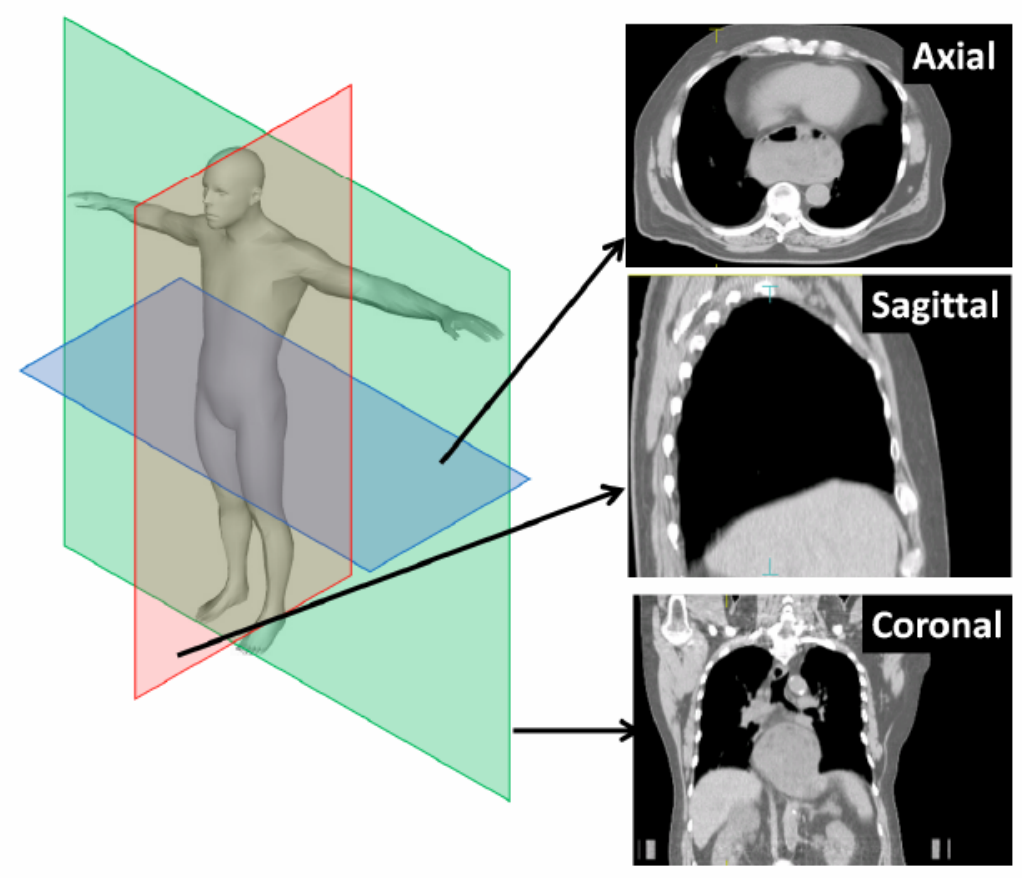

Figure 1.2: Illustration of the axial (top right), sagittal (middle right) and coronal (bottom right) views of a CT scan. The CT images show the thorax of one of the patients included in this study.

scans are used for the delineation of the tumour and surrounding healthy organs, for radiation dose calculations, and for verification of patient position immediately before treatment. CT scans can be viewed in the axial, sagittal and/or coronal views (see Figure 1.2). In the following chapters, these different CT scan views are extensively used to present the results visually. An axial view gives anatomical information in the plane formed by the combination of the left-right and anterior-posterior (or front-back) directions of the patient. A sagittal view gives anatomical information in the plane formed by the combination of the anterior-posterior and superior-inferior directions (superior means towards the head and inferior means towards the feet). A coronal view gives anatomical information in the plane formed by the combination of the leftright and superior-inferior directions. From the CT scans, Digitally Reconstructed Radiographs (DRRs) can be generated. DRR images (examples shown in Figure 1.3) are frequently used in this study. DRRs are the counterpart of the traditional radiographs. 

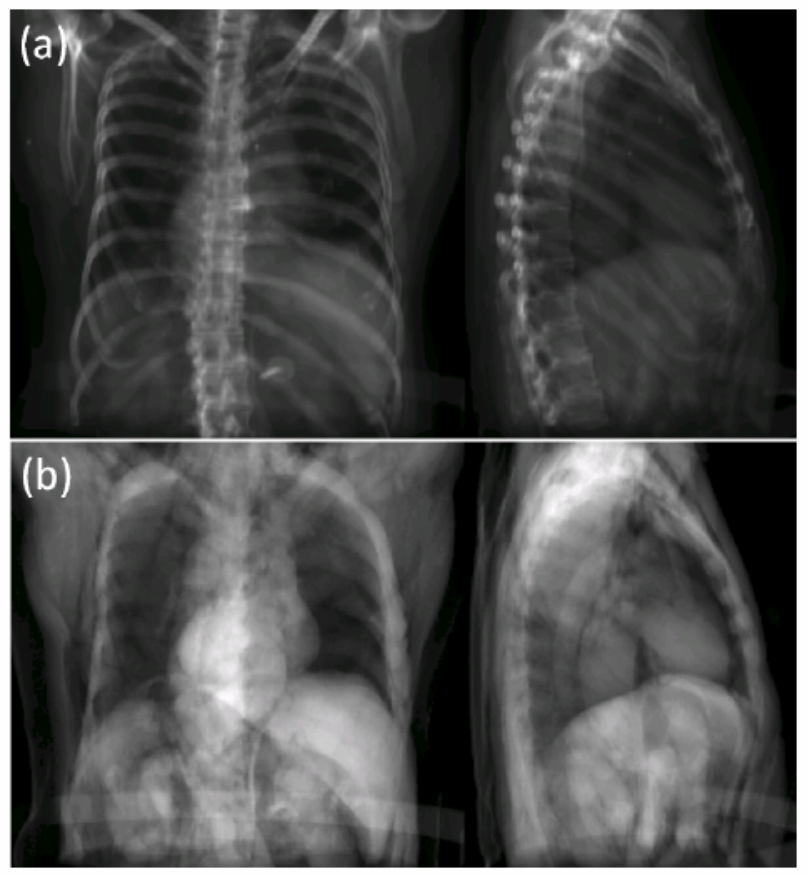

Figure 1.3: Examples of orthogonal DRRs of a patient using two different window and level settings focusing on showing (a) bony structures and (b) soft tissues.

Multiple imaging modalities can be used to help in tumour and healthy organ delineation. Positron Emission Tomography (PET) and Magnetic Resonance Imaging (MRI) scans are used to improve the accuracy of defining the tumour location and extent. ${ }^{5,6}$ These secondary images are registered with the treatment planning CT scan with focus on the volume where the tumour and the surrounding healthy organs are located.

Different target volumes are delineated on the treatment planning CT, as shown in Figure 1.4. The Gross Tumor Volume (GTV) is defined as the visible or palpable extent of the cancer growth. ${ }^{3,7}$ If subclinical disease is present, a Clinical Target Volume (CTV) is delineated. The CTV contains the GTV and/or subclinical malignant disease that should be eliminated. This volume should be treated adequately in order to achieve the aim of RT. ${ }^{3,7}$ To account for different uncertainties during treatment, different margins are added to the CTV to create the treatment volume. An Internal Margin (IM) is 


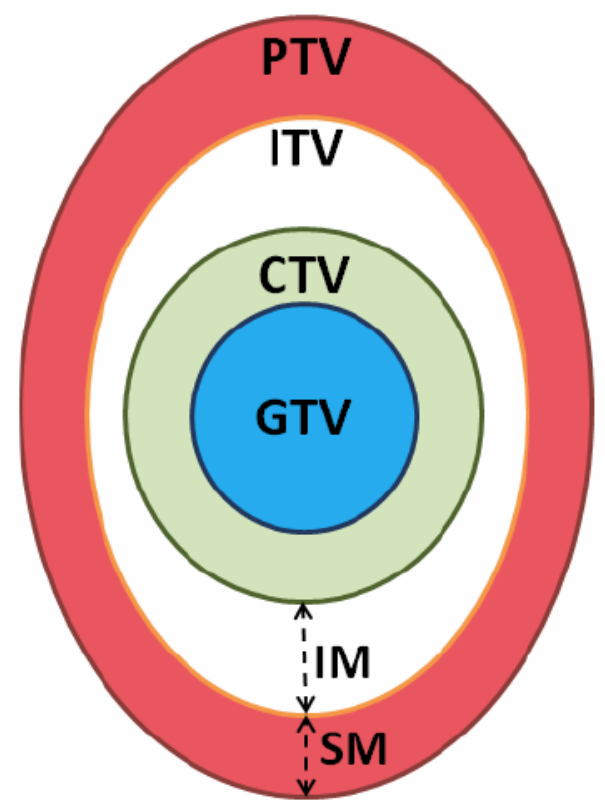

Figure 1.4: Diagram showing the GTV, CTV, ITV, and PTV. The CTV is expanded to the ITV by adding an Internal Margin (IM) to account for plausible motion during treatment. A Setup Margin (SM) is added to the ITV to account for setup uncertainties, creating the PTV.

added to the CTV to account for physiological movements and changes in the size and shape of the CTV during treatment (e.g., breathing for tumors in the lung). The volume that includes both the CTV and the IM is called the Internal Target Volume (ITV). ${ }^{3,7}$ Furthermore, a Setup Margin (SM) is added to the ITV to account for uncertainties in patient positioning and in the machine components during the treatment. A Planning Target Volume (PTV) contains the ITV plus the SM, and it is the volume used for RT planning. ${ }^{3,7}$ The PTV is the volume used for RT planning. With the knowledge of the PTV extent, the LINAC beams are positioned and shaped to optimally deliver the prescription dose $(\mathrm{Rx})$ to the target while sparing surrounding healthy organs as much as achievable. Organs at risk (OARs) are healthy organs whose tolerances to radiation affect the dose that can be safely delivered to the tumour. ${ }^{3,7}$

The second step in the EBRT process is the treatment planning. A radiation beam 
model of the LINAC treatment beam is created during commissioning. A specialized Treatment Planning System (TPS) uses the LINAC beam model to simulate LINAC geometry, and uses the simulation CT scan to calculate the radiation dose delivered to the patient. The CT values (also known as Hounsfield Units, or HU) of the voxels in the simulation scans are converted into Electron Densities, ED (electrons per cubic centimetre) using a CT scanner specific conversion curve (HU-to-ED curve). These electron densities are used to create the patient model used in the dose calculations. Within the TPS, there are tools to assess the quality of the radiation treatment such as the 3D dose distribution and the Dose Volume Histogram (or DVH - a DVH contains information about how much percent of the volume of a structure receives a certain dose level). The plan assessment metrics used in this study are discussed in Section 2.7.

The third step in the EBRT process is the treatment delivery. Once the treatment plan is approved by the radiation oncologist and the medical physicist, the plan information (i.e., treatment beam shapes and photon beam fluence) are transferred to the treatment machine. The Monitor Unit (MU) is a physical quantity used to refer to the machine output. A typical LINAC calibration is to deliver $1 \mathrm{cGy}$ of radiation dose per MU in standard calibration conditions ( 5 or $10 \mathrm{~cm}$ depth in a water tank, with a square radiation field size of $10 \times 10 \mathrm{~cm}^{2}$, and a distance between the radiation source and the point of measurements of $100 \mathrm{~cm}) .{ }^{8}$ Verification of the treatment plan is called patient-specific Quality Assurance (QA), and is carried out before the actual treatment using a combination of the following tools: (1) delivering the radiation treatment plan on a pseudo-patient (e.g., a phantom with material of comparable radiation properties to water or tissue) ${ }^{9}$ (2) performing plan output or MU checks using an independent software, ${ }^{9-11}$ and/or, (3) analyzing the LINAC log files of the delivery. ${ }^{12}$ These measurements are compared to the dose information from the TPS. In 
this study, the machine performance with different techniques is assessed by delivering the treatment plans and analyzing the LINAC log files. After passing the verification tests, the plan is approved for treatment. Pre-treatment imaging is used to ensure that the patient position matches the position during simulation. Imaging devices used for pre-treatment position verification are typically on-board portal imaging devices or Cone-Beam Computed Tomography (CBCT).

The EBRT process, from the point of CT scanning to patient-specific QA, typically takes a few days to two weeks. For cases in which a patient requires prompt treatment, the process is typically shortened by choosing the simplest treatment technique.

\subsection{Fractionation}

The prescribed radiation dose in EBRT is typically divided into smaller doses, or fractions, delivered in different sessions to allow the irradiated normal tissues to repair from radiation damage. ${ }^{13}$ The time between each fraction should be long enough for normal tissue repair, but short enough to not allow for tumour repopulation. ${ }^{13}$ Fractionation depends on the disease diagnosis and the intent of treatment. Conventional fractionation is one session per day, five days a week spanning up to eight weeks. ${ }^{13}$ Treatments with conventional fractionation usually take 15 to 30 minutes, including the patient setup time and the beam-on time. Hypofractionation is another commonly followed fractionation in which a higher dose per fraction is delivered in a smaller number of fractions. ${ }^{13,14}$ Hypofractionation can be used for both curative and palliative intent treatments. ${ }^{13,14}$ Examples of hypofractionated curative treatments are Stereotactic Radiosurgery (SRS) for intra-cranial tumors and Stereotactic Body Radiation Therapy (SBRT) - also known as Stereotactic Ablative Radiotherapy (SABR) - for extra-cranial tumors. Stereotactic treatment techniques use special immobilization devices that al- 
low for more accurate positioning of the patient during treatment. ${ }^{15}$ Moreover, these techniques typically use image guidance during treatment, ${ }^{16}$ which is not typically done in conventional treatment techniques. Such stringent geometric accuracy allows for a higher daily dose to be delivered to the tumour (e.g., 6000 cGy in five fractions for lung SBRT). Hypofractionation is also implemented for palliative treatments (e.g., 800 cGy in one fraction) for symptom management. ${ }^{17}$ There are also moderate hypofractionation such as 2000 cGy in five fractions. The treatment plans used in this study follow conventional fractionation, moderate hypofractionation and hypofractionation (i.e., SBRT treatments). The radiation tolerance values for OARs differ for different fractionations and are determined from various levels of clinical trials for each type of cancer disease and dose fractionation. In the absence of clinical data, the OAR tolerance values for a specific dose fractionation are inferred from biological considerations such as biologically equivalent dose calculations as a first-order approximation. ${ }^{18-20}$ Tolerance values for relevant OARs are discussed in Chapter 2.

\subsection{CT simulation of lung patients}

As mentioned in Section 1.1, CT simulation is the first step in the EBRT process. For many treatments, different immobilization devices are used (examples are shown in Figure 1.5) to restrict patient motion and to ensure that the patient position during simulation is reproduced during treatment. ${ }^{9,21}$ However, there are cases where motion is unavoidable, like bladder and rectal filling, swallowing, and motion caused by breathing. This section focuses on the simulation process for patients with lung tumour and how breathing motion is accounted for in the treatment delivery and in the radiation dose calculations. Lung SBRT cases are included in this study to evaluate plan quality and robustness of the different treatment techniques. 


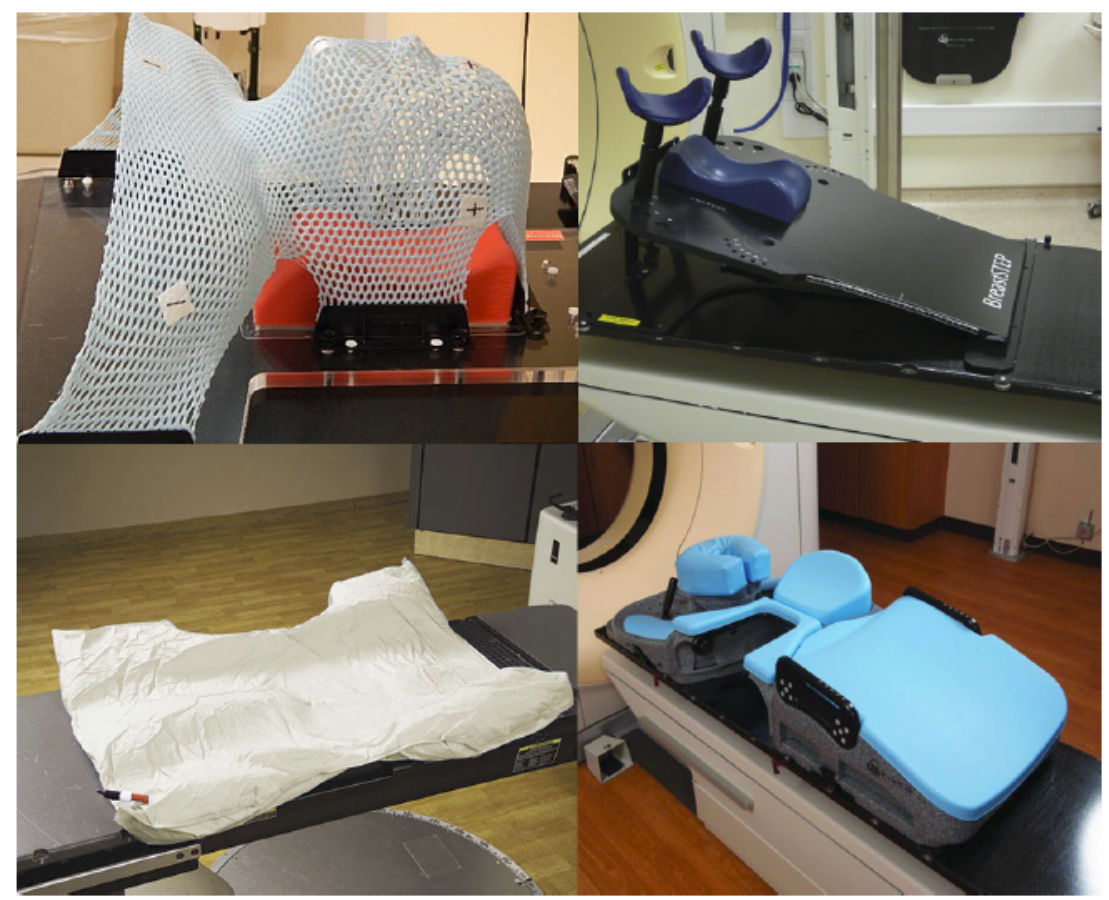

Figure 1.5: Examples of immobilization devices used in EBRT.

When treating patients with lung tumors, the goal is to treat the tumour while excluding the surrounding healthy tissues in the presence of breathing motion. Gating is one of the approaches closest to achieving this goal. ${ }^{22,23}$ With gating, the patient motion is tracked during treatment and a signal is sent to the LINAC to irradiate only when the tumour is in the range of locations for which the treatment planning is done. But currently the most straightforward approach, which is the most commonly used clinically, is to account for the patient-specific range of motion in the internal margin (see Figure 1.4) during treatment planning. ${ }^{3,7,22,23}$ It is important to identify carefully the internal margin. An insufficient internal margin leads to tumour underdosage since parts of the PTV fall outside the radiation beam during parts of the breathing motion, and an excessive internal margin leads to unnecessary irradiation of surrounding healthy tissues. Analysis of the breathing motion of different cohorts of patients showed lung tumour motion amplitude ranging from 0 to $34 \mathrm{~mm}$ in the superior-inferior direction. ${ }^{24}$ One of the patient cohorts showed lung tumour motion reaching an amplitude of $50 \mathrm{~mm}$ 
in the superior-inferior direction. ${ }^{25}$ The typical breathing period is from three to six seconds. ${ }^{26}$ The accuracy of internal margin for lung cases is particularly important for stereotactic treatments because of the small target size, the steep dose gradients, and the larger dose per fraction. ${ }^{27}$

A CT scan acquired while the patient is breathing normally (or "freely") is typically used for treatment planning. However, this free-breathing scan does not necessarily represent the average target position since each projection of the scan is imaged at a different breathing phase away from the actual average target position. ${ }^{28}$ The most accurate way in identifying the internal margin requires the use of Four-Dimensional Computed Tomography (4DCT). ${ }^{23}$ During 4DCT, as the patient is scanned, the respiration signal is simultaneously acquired. Once the images are acquired, they are post-processed into individual 3D image sets according to the respiratory phase at the time each image was acquired. ${ }^{29}$ The breathing cycle is monitored with a bellows-based system that is strapped around the patient abdomen and is stretched and contracted during breathing. The internal pressure and the amplification of the bellows are monitored in a computer, and this information is synchronized to the CT data. Each projection image is assigned with a breathing amplitude and breathing rate. Binning of the 4DCT data provides anatomically accurate images of the lungs and other surrounding structures at different breathing phases.

In the 4DCT unit used at The Ottawa Hospital Cancer Centre (TOHCC), ten series of images are generated that correspond to ten breathing phases within a full breathing cycle of a patient. If the range of motion of the tumour seen from the scans is larger than $1 \mathrm{~cm}$, an abdominal compression device is placed on the patient, and the whole 4DCT process is repeated. Various methods of creating an ITV from a 4DCT dataset have been reported. ${ }^{27,30}$ The first, most robust, but time consuming for clinical use, is 
to delineate the GTV and/or CTV on all the ten scans of the respiratory cycle from the 4DCT dataset. An ITV is created as the union of the GTVs and/or CTVs from each of the ten phases. An alternative method is to use a Maximum Intensity Projection (MIP) image. This is a single CT image created from the 4DCT dataset, and it reflects the highest intensity value encountered in each pixel throughout the respiratory cycle. ${ }^{30}$ The information from the MIP scan is used at TOHCC to determine the internal margin. The average and the MIP scans are fused or overlaid on each other in the TPS. The average scan is also a single image created from the 4DCT dataset, and it reflects the pixel-by-pixel average of the scans of the ten breathing phases. Using the information from the MIP scan, the ITV is contoured on the average scan such that it encompasses the visible range of GTV. The GTV-to-ITV expansion values are used in Chapter 7 to assess robustness of the investigated treatment techniques against typical breathing motion. The average scan is the primary image set where the dose calculations are performed at TOHCC and in this study.

\subsection{LINAC in photon mode}

A typical EBRT unit (see Figure 1.1) consists of: (1) a LINAC, where the electron acceleration then deceleration occurs, and the shaping of the resulting clinical photon beam follows, (2) a flat couch, where the patient is positioned for treatment, and, (3) an imaging device attached to the LINAC, which is used to verify patient position before treatment. The LINAC gantry head can rotate in a full arc around the patient, allowing for the radiation beam to come from any angle. The point in space around which all the treatment unit components (gantry, collimation system, treatment couch, imaging system) rotate is called the isocentre. The isocentre is typically $100 \mathrm{~cm}$ away from the radiation source. 
Before exiting the LINAC gantry head, the photon beam may be altered by passing through a conical shape flattening filter which produces a flat dose distribution at a certain depth in a water phantom. There are LINAC designs that do not incorporate flattening filters. Current LINAC designs use two or three collimation devices to shape the photon beams, namely the primary, secondary and Multi-Leaf Collimators (MLCs). The primary collimator is a conical opening between the target and the flattening filter, and it defines the largest available circular field size. The secondary collimators may consist of two or four blocks, forming the upper and/or lower jaws of the collimators. They can provide rectangular or square fields at the LINAC isocentre, with sides of up to 30 or $40 \mathrm{~cm}$. MLCs consist of leaves that can independently move at different speeds and collectively produce different shapes (e.g., conforming the beam shape to the shape of the target). This allows for blocking healthy tissues that could have unnecessarily been exposed in the rectangular field of the secondary collimator.

The LINAC used in this study is the Elekta Synergy/Infinity system with an Agility $^{\mathrm{TM}}$ collimation assembly (Elekta, Stockholm, Sweden), shown in Figure 1.6. The Agility collimation system consists of one pair of jaws in one direction and in the other direction is a pair of dynamic leaf guides or carriages. The carriage does not attenuate the photon beam; its main function is to drive the MLC assembly. The MLC assembly has 160 leaves (80 pairs) of $5 \mathrm{~mm}$ width (projected at the isocentre) covering fields up to $40 \times 40 \mathrm{~cm}^{2}$ (also projected at the isocentre). The carriages and the MLC leaves move independently of each other. The composite speed of a particular MLC leaf is the sum of the carriage speed plus the physical speed of that particular leaf. 


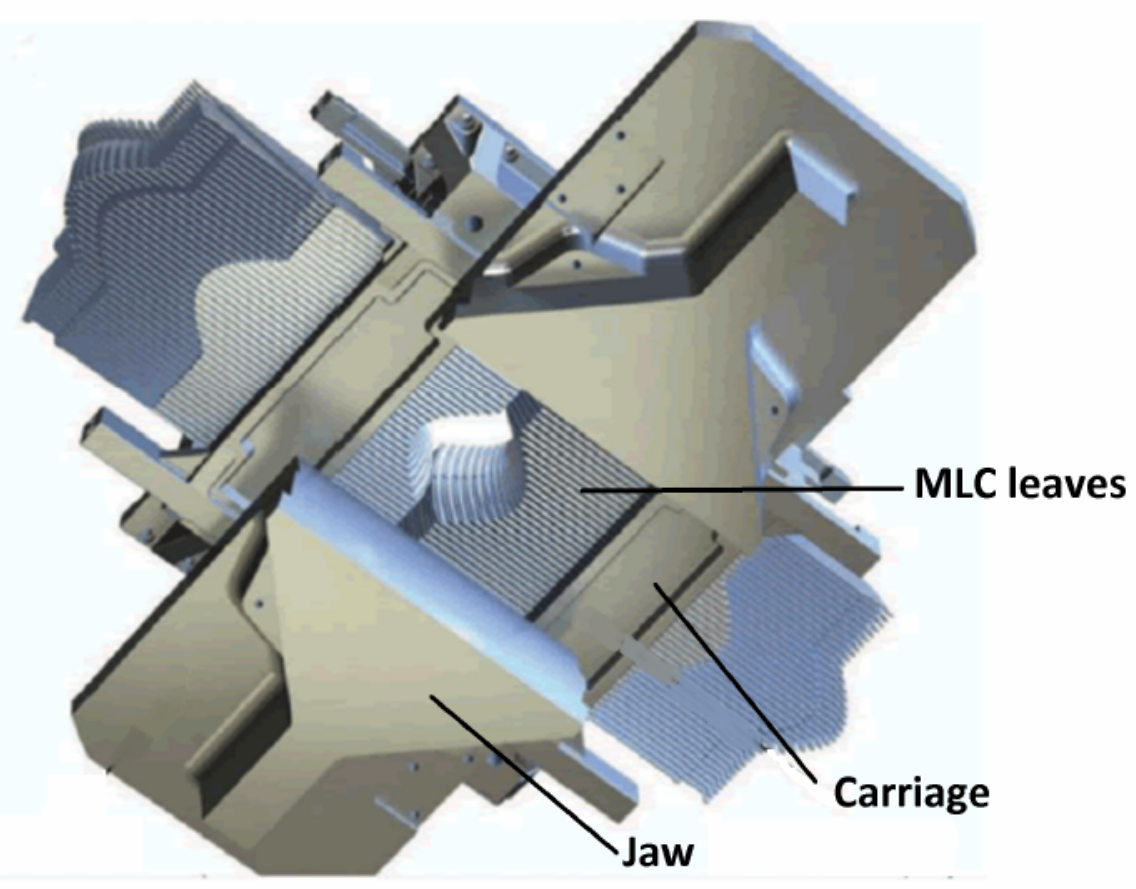

Figure 1.6: The Elekta Agility ${ }^{\mathrm{TM}}$ collimation system assembly installed in the LINACs used in this study.

\subsection{Treatment techniques in photon mode}

Photon beam treatments are classified into different techniques depending on the characteristics of the different LINAC components during radiation beam-on time.

Generating treatment plans for these techniques is through either forward or inverse planning. In forward planning, the geometry for each beam (gantry angle, collimator angle, couch angle, jaw opening or field size, and MLC positions) are set manually before performing the radiation dose calculations. The beam geometries and beam weighting are adjusted manually until the optimal dose distribution is achieved. For the treatment techniques that can maximize the use of the degrees of freedom available on the LINAC, manually trying the different combinations of beam geometries by trialand-error is impractical, and inverse planning is the usual treatment planning approach. In inverse planning, the treatment goals are fed to an optimizer in the TPS. These goals 
include objectives (which indicate the desired dose to the tumour), and constraints (which limit the dose to the surrounding healthy organs). In the Monaco TPS (which is the TPS used in this study), there are three steps in the optimization process to achieve a clinically acceptable plan. During step 1, the optimizer generates the ideal photon beam fluences that minimize the composite cost function (weighted sum of the objectives and constraints). Then in step 2, the optimizer translates these ideal photon fluences from step 1 into deliverable beam apertures that respect the physical LINAC component limitations such as the achievable range of dose rate, gantry speed, jaw speed, MLC speed, and carriage speed (both the minimum and maximum limits). During step 3, the generated beam apertures from step 2 are refined through Segment Shape Optimization (SSO), mainly to improve plan quality. SSO is one of the main focuses of this study, and is discussed further in the following chapters. Depending on the treatment technique, a combination of these three steps is implemented during optimization in the Monaco TPS, which is discussed later in this study.

In the following subsections, the commonly used photon beam treatment techniques are described in terms of LINAC component characteristics during beam-on time and the treatment planning approach for each technique. A number of these treatment techniques are used extensively in this study.

\subsubsection{Three-Dimensional Conformal Radiation Therapy (3DCRT)}

Three-Dimensional Conformal Radiation Therapy (3DCRT) typically consists of a few beams with static LINAC components during beam-on time. The LINAC components (gantry, jaw, colllimator, couch and MLC leaves) are moved to their planned position before the radiation beam is turned on for each beam. Typically, the MLC leaves of the main beams follow the shape of the target with some margin (the margin is to 
compensate for the blurring effect at the edge of the beam - called beam penumbra). ${ }^{1}$ For a given beam within a 3DCRT plan, the dose rate is constant, and the photon intensity reaching the patient is uniform across its open field. Different beams within a 3DCRT plan can have different weights. Given the manageable number of beams and the static positions of LINAC components during a 3DCRT delivery, 3DCRT plans are generated through forward planning. Examples of the most simple 3DCRT beam arrangements are single beam, parallel-opposed beams and four-field box.

The shape of the delivered dose is inherent to the beam geometries (see Figure 1.7). In a single beam, the highest doses (or hotspots) are located near the entrance of the beam, and the radiation is attenuated as it gets deeper in the patient. On the other hand, a rectangular dose distribution is produced when using two orthogonal pairs of parallel-opposed beams (a four-field box), which does not produce hotspots at the entrance because the photon fluence is divided among the four beams. In 3DCRT, the number of beams depends on the size, shape and location of the target relative to the nearest OARs. The larger the number of beams, the more the resulting dose distributions gets conformal to the target. Depending on the size of the patient and the heterogeneities of the surrounding tissues, if the maximum dose with open fields would exceed the recommended $107 \%$ limit, ${ }^{3,7}$ subfields (also called fields-in-field) are used (practically, planning goals aim for a maximum dose of 105\%). Subfields are created to block parts of the beam shape where the hotspots are located, and are given enough weight to reduce the hotspots to the recommended level while maintaining the original target dose coverage. 

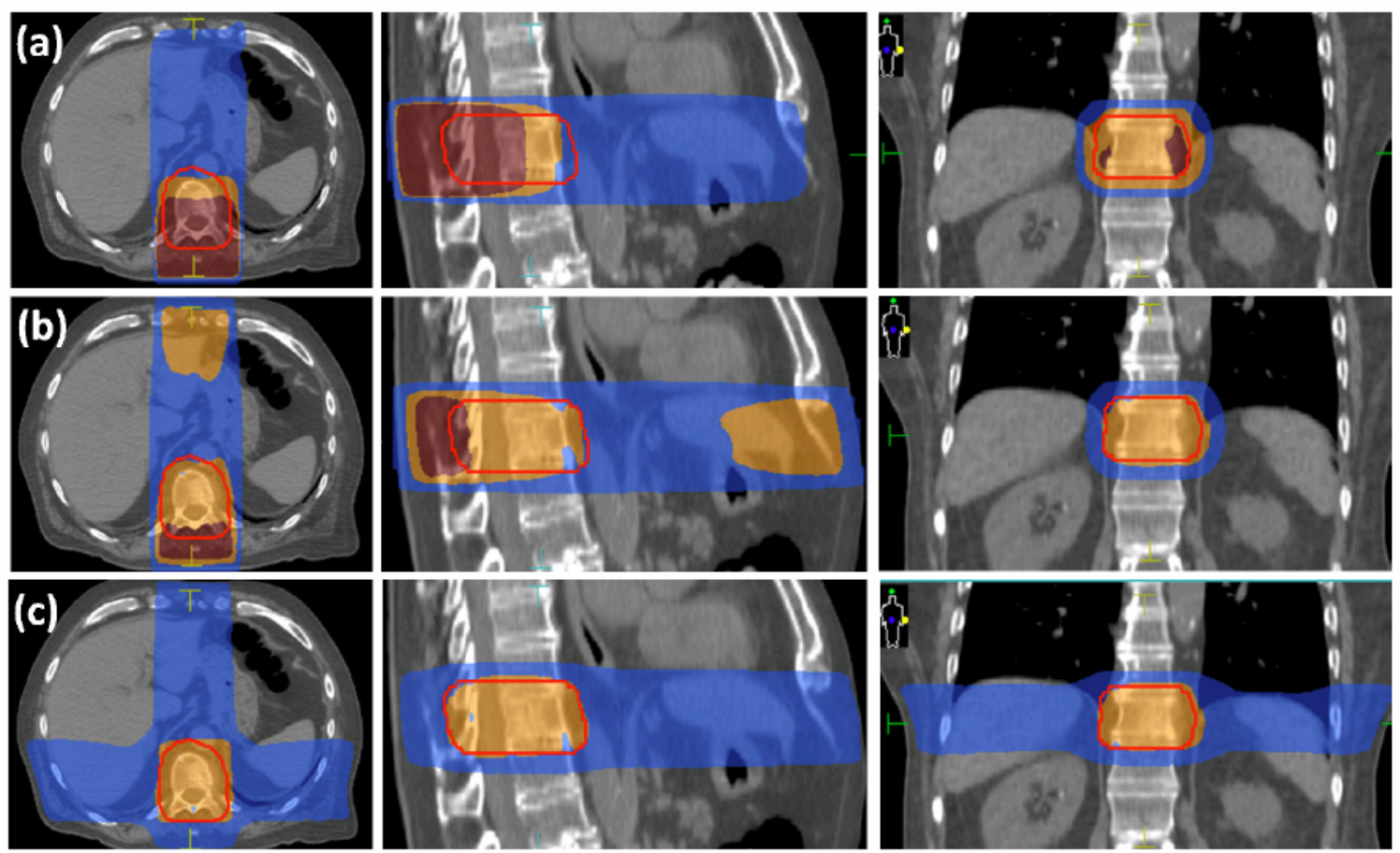

Figure 1.7: For an example palliative spine patient, the figure shows dose distributions in the axial (left), sagittal (middle) and coronal (right) CT views for different beam arrangements: (a) a single posterior beam, (b) parallel-opposed beams and (c) a four-field box. The color-washes represent the following percent of the Rx: $50 \%$ (blue), $100 \%$ (orange) and $105 \%$ (brown). Note the large hot spot in (a), and the undesirable irradiation of the liver and small bowel to the full $\mathrm{Rx}$ in (b).

\subsubsection{Intensity Modulated Radiation Therapy (IMRT)}

The main differences between Intensity Modulated Radiation Therapy (IMRT) and 3DCRT are that there is typically a larger number of beams at each gantry position, and that the MLC leaves of each beam can move during the beam-on time, which effectively creates a cumulative non-uniform photon fluence reaching the patient from a given beam at a given gantry angle. ${ }^{9,31,32}$ The MLC leaves during IMRT do not necessarily follow the shape of the target. The MLC leaf positions are generated through inverse planning, where the leaves can shadow over the target given that the final dose distributions respect the plan objectives and constraints, and respects the physical 
performance limits of different LINAC components. With the high degree of radiation dose conformality achievable with IMRT, it has been the standard treatment technique for complex cases due to evidence of reduced OAR toxicities. ${ }^{33,34}$

\subsubsection{Classical Dynamic Conformal Arc Therapy (cDCAT)}

The basic concept of arc therapy is the delivery of radiation from a continuous rotation of the LINAC gantry head which allows the patient to be treated from the full $360^{\circ}$ angle range. During a classical Dynamic Conformal Arc Therapy (cDCAT) delivery, the gantry rotates around the patient with a single gantry speed and a single dose rate for the entire arc while the MLC leaves continuously conform to the shape of the target. The beam is on throughout the $360^{\circ}$ arc delivery. Each arc is subdivided into segments and each segment has defined starting and ending positions (i.e., control points) for the gantry head, jaws and MLC leaves. The couch and collimator angles stay the same for the entire arc delivery.

Planning cDCAT is considered somewhat forward planning since the beam geometries such as arc range, number of segments, jaw positions and MLC shapes have to be specified by the user. These characteristics of cDCAT make it a useful technique in treating very small and regularly shaped tumors of lung ${ }^{35-37}$ and brain ${ }^{38-44}$ stereotactic treatments. The term "classical" is coined for the purpose of this study to differentiate between the earlier implementations of DCAT (called cDCAT in this study) and a modern, more flexible implementation of DCAT, which is the subject of this study and the acronym mDCAT is coined for it. 


\subsubsection{Volumetric modulated arc therapy (VMAT)}

Although Volumetric Modulated Arc Therapy (VMAT) is an arc delivery like cDCAT, the main differences between cDCAT and VMAT deliveries are the dose rates of the segments relative to one another, the modulation within a segment, and the gantry speed. In VMAT, the radiation beam is ON throughout the arc delivery and the gantry has a variable speed as it rotates around the patient. ${ }^{45}$ The arc is divided into segments, and the cumulative photon fluence for each segment is non-uniform because the individual MLC leaves can move at different speeds across the segment, substantially shadowing over the target while the radiation beam is ON. Shown in Figure 1.8 are examples of the photon fluence reaching the patient for a particular segment within a VMAT and a cDCAT plan, as well as the starting and ending MLC leaf positions of that particular segment. With the degree of modulation that VMAT has, it is a common technique used to treat complex cases, particularly when an OAR overlaps with or is very close to the tumour. The main advantage of VMAT versus IMRT is the added degrees of freedom offered by the rotating motion of the LINAC gantry during beam-on time. An

additional advantage is the improvement in treatment delivery efficiency. ${ }^{46,47}$ VMAT is one of the most commonly used treatment technique at this time. VMAT plans, like IMRT plans, are produced using inverse planning.

\subsubsection{Special techniques}

Helical Tomotherapy and CyberKnife are treatment techniques that use different designs of LINACs.

With helical Tomotherapy, the LINAC is mounted on a doughnut-shaped gantry 

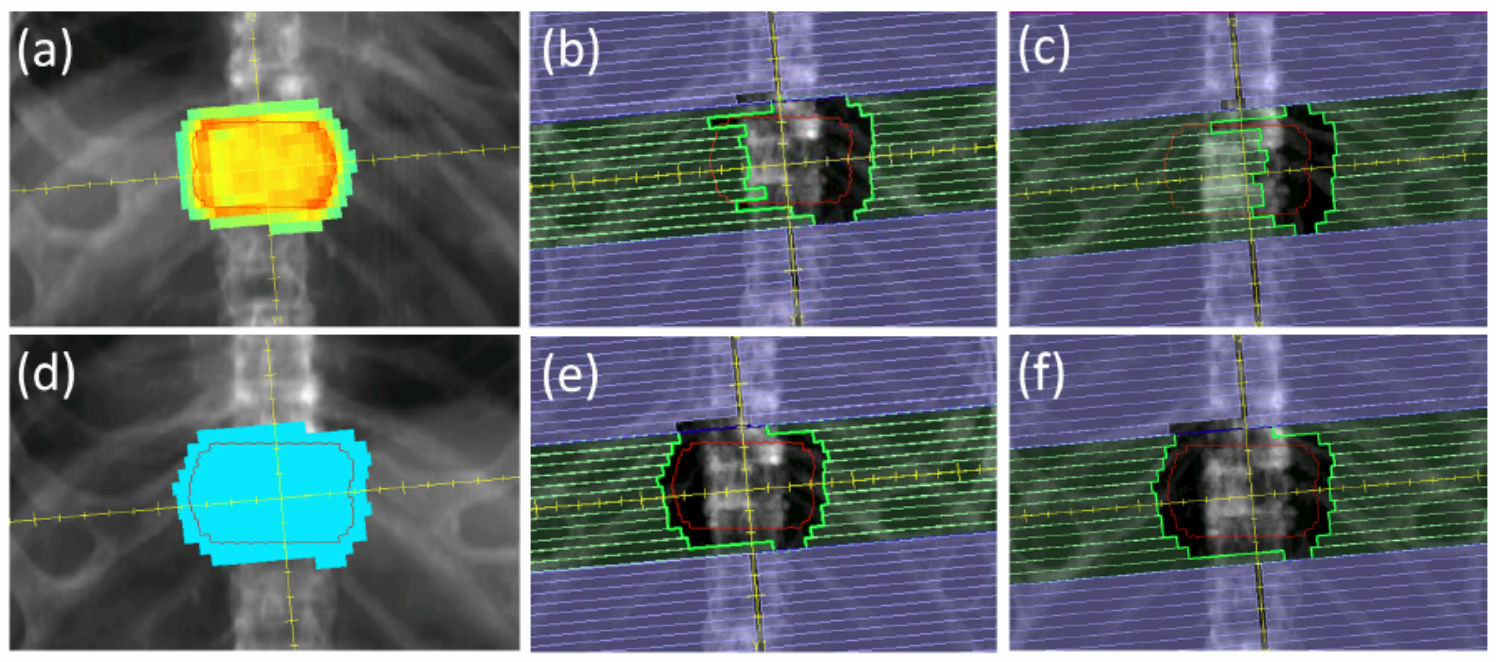

Figure 1.8: Example of the photon fluence and segment shapes for a beam segment in a VMAT and a cDCAT plan for one of the patients included in this study. VMAT has a non-uniform photon fluence as shown in (a), and the start and the end positions of the beam segment are shown in (b) and (c), respectively. The uniform photon fluence of cDCAT is shown in (d), and the corresponding start and end positions of the beam segments shown in (e) and (f), respectively. Note that the MLC leaves move over the target (contoured in red) during the movement from (b) to (c), but not during the movement from (e) to (f), which leads to the fluence patterns shown in (a) and (d).

that looks similar to a conventional CT-scanner, allowing the LINAC to rotate around the patient while the treatment couch moves into the bore. During treatment, the couch advances the patient through the bore so that the radiation dose is delivered in a helical geometry around the target volume. Beam collimation is accomplished with a computer controlled MLC assembly, also on the rotating gantry, that has two sets of interlaced leaves that rapidly move in and out of the beam in a binary fashion to constantly modulate the photon fluence reaching the patient as the LINAC rotates around the patient. ${ }^{48,49}$

With CyberKnife, a very narrow beam of radiation is delivered by a robotic arm that can come from any direction (except from under the treatment couch). Circular fields with diameters from $5 \mathrm{~mm}$ to $60 \mathrm{~mm}$ are produced with CyberKnife using either 
fixed cone collimators or an iris collimator system. Fixed collimators are cones with discrete diameter openings that are attached to the end of the robotic arm, while an iris collimator can produce circular fields with different diameters. In a CyberKnife treatment, there is more frequent imaging during treatment compared to the other EBRT techniques, resulting in better tumour tracking. Because of this high localization accuracy, radiation dose can be escalated and can be delivered in a single or few sessions.

\subsection{Motivation and contributions}

Motivation: This work was motivated by the recent release of a modern implementation of DCAT (coined the acronym mDCAT in this study) by one of the widely used TPSs - Monaco TM (Elekta, Stockholm, Sweden). Monaco is the TPS used at TOHCC. mDCAT allows for dose rate variation and moderate beam modulation, which make mDCAT more flexible than cDCAT but not as flexible as VMAT. There are studies that presented the performance of the cDCAT (constant dose rate and photon beam conforming to the shape of the target) and they are discussed in Section 1.7. However, there are no studies exploring mDCAT. Additionally, previous studies have only looked at the plan quality achievable by cDCAT but have not evaluated the robustness of DCAT in general versus VMAT against machine and patient uncertainties. Robustness is an important factor when comparing different treatment techniques, particularly for small targets, steep dose gradients and high dose per fraction.

Another motivation for this study is that, with the existing treatment techniques, the delivered dose can be conformal but at the expense of treatment planning efficiency (such as with VMAT), or the treatment planning can be made more efficient but at the expense of target dose conformality (such as with 3DCRT), therefore mDCAT may be a good compromise between efficiency and conformality, which is particularly relevant 
for cases that require high-quality and yet rapid-access palliation. mDCAT can be a key ingredient in a new paradigm in rapid-access palliation where the entire conformal EBRT process can be done in less than an hour.

Contributions: The two main contributions of this study are: (1) it provides a comprehensive characterization of a modern implementation of DCAT, and, (2) it explores the potential gains from using it in the current RT landscape by comparing its performance against the most commonly used techniques at present - 3DCRT and VMAT. The comparison is in terms of plan optimization and treatment delivery efficiency, plan quality, and plan robustness. Robustness is a measure of the stability of the actual dose delivered to the patient against uncertainties during treatment delivery. The uncertainties can be in the LINAC components and/or in the patient/tumour position during delivery. Such uncertainties can be systematic and/or random and they can be of typical or extreme magnitude. This study contributes to the understanding of the impact of all such uncertainties on plan robustness.

\subsection{Review of previous studies on classical DCAT (cDCAT)}

Compared with LINAC-based IMRT, cDCAT was shown to offer comparable ${ }^{38}$ to bet$\operatorname{ter}^{40}$ coverage for large and small stereotactic single brain targets, respectively. For cases in which the target overlapped with or was nearby OARs, IMRT offered better sparing of the OARs, reducing doses to the OARs up to about $30 \%$ compared to those achievable with cDCAT. ${ }^{41,50}$ For most cases, using the standard plan quality metrics, cDCAT was the preferred technique versus IMRT particularly because of the less total MUs required to treat (by a factor of two to three), resulting in less integral body dose (which could reduce the probability of radiation-induced secondary cancers). ${ }^{38,40,41,50}$ In comparison to VMAT, cDCAT required lower total MUs and offered better sparing 
of normal brain tissues, however, VMAT demonstrated better target coverage and dose conformity than cDCAT due to the larger number of degrees of freedom in a VMAT treatment. ${ }^{44}$ For cases in which VMAT or IMRT offered better organ sparing, this was attributed to the inverse planning capabilities of VMAT and IMRT planning compared to the forward planning approach for cDCAT. ${ }^{44,50}$

A study by Rauschenbach et $a l^{36}$ compared 3DCRT, cDCAT and VMAT for lung SBRT cases. The target sizes ranged from 12.7 to $190.7 \mathrm{~cm}^{3}$. cDCAT and VMAT both offered better target coverage compared to 3DCRT, but VMAT was shown to be always superior in sparing OARs, reducing the mean dose to the healthy lungs by up to $13 \%{ }^{36}$ cDCAT had the lowest total MUs which required only $52 \%$ of the MUs delivered with VMAT, followed by 3DCRT which required $71 \%$ of the MUs delivered with VMAT. Takeda et $a l^{35}$ showed that high conformality was achievable by cDCAT for lung SBRT cases with healthy lung dose within the tolerance values. Morales-Paliza et $a l^{50}$ also looked at the performance of cDCAT for other disease sites, namely, neck, spine and prostate tumors. For both the neck tumour patient and the spine tumour patient investigated, IMRT offered slightly better target coverage compared to cDCAT, but most OARs were spared comparable to or better than what was achievable with IMRT. The average total MU for cDCAT was only one-third of the average total MU for IMRT. For the twelve prostate cases investigated, cDCAT and IMRT offered comparable dose coverage and OAR sparing. 3DCRT, on the other hand, could not achieve the same level of OAR sparing offered by cDCAT and IMRT.

cDCAT was also compared with helical Tomotherapy for one type of brain tumors. ${ }^{43}$ Both cDCAT and helical Tomotherapy had clinically acceptable target coverage, with helical Tomotherapy offering slightly better target coverage. Most OAR maximum doses were lower with helical Tomotherapy, however, the dose gradient outside of the 
target was better with cDCAT. Helical Tomotherapy consumed ten times more MUs compared to cDCAT, resulting to longer treatment times $(33.2 \pm 7.4$ minutes versus $4.6 \pm 0.9$ minutes)

\subsection{Thesis organization}

This thesis is organized as follows. In Chapters 2, the treatment planning (as implemented in the Monaco TPS) and the overall methods are described. In the second half of Chapter 2, the choice of patients used in this study and its relevance to the goals of this work are explained. Beyond chapter 2, the methods specific to each chapter are described within, followed by the results. In Chapter 3, the methods and results of the characterization of mDCAT in the Monaco TPS are discussed. In Chapter 4, the plan optimization and treatment delivery efficiency of mDCAT is compared with VMAT. In Chapter 5, the plan quality achievable with mDCAT is compared against 3DCRT and VMAT (which are two of the most commonly used EBRT techniques). In Chapters 6 and 7, the robustness of mDCAT against treatment uncertainties is assessed by artificially introducing machine errors and patient motion (breathing), respectively. 


\section{Chapter 2}

\section{Treatment planning}

In this chapter, the tools and features that were used in the Monaco TPS version 5.11 are described, followed by discussion of the rationale behind the selection of patients included in this study, then a description of the treatment planning methodology for 3DCRT, VMAT and mDCAT.

\section{$2.1 \quad$ Forward planning}

In forward planning, the geometry for each beam (gantry head angle, collimator angle, couch angle, jaw opening for field size, and MLC positions) are set manually before performing the radiation dose calculations. ${ }^{51}$ One of the most common techniques planned with forward planning is 3DCRT, which was discussed in Section 1.5.1. Shown in Figure 2.1 are the tabs in the Monaco TPS where the beam details can be manually modified. Defining the MLC leaf positions of the main photon beams is typically done by conforming the MLC leaves around the target volume with some added margin to account for the beam penumbra at the edge of the field. The MLC leaf positions can also be manually modified (moving MLC leaves one at a time) if needed, such as in creating subfields. 


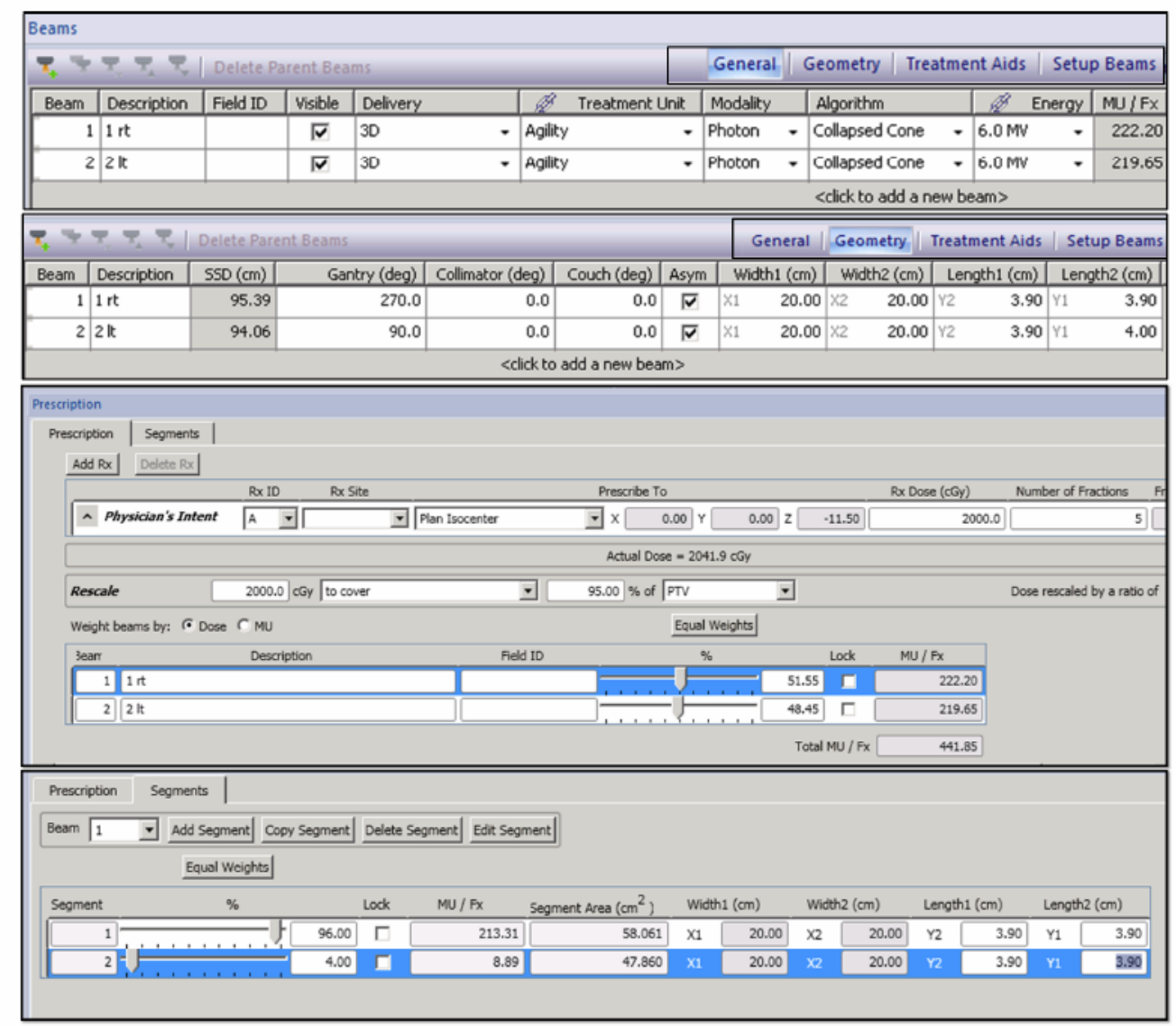

Figure 2.1: Tabs in the Monaco TPS that allow for entering the beam geometries, Rx and beam weights. The bottom panel shows the breakdown of beam "1 rt" into a main segment and a subfield segment.

\section{$2.2 \quad$ Inverse planning}

In inverse planning, the treatment goals (namely the objectives and constraints) are fed to the optimizer to produce a treatment plan. Inverse planning is extensively used in this study to generate VMAT and mDCAT plans. Only the details relevant to this study are discussed below.

\subsubsection{Optimization mode}

Optimization in the Monaco TPS can be set to either "constrained" or "Pareto" mode. Setting the optimization to "constrained" mode places the priority on meeting the OAR 
constraints at the risk of not meeting the target volume objective. ${ }^{52}$ An optimization in "Pareto" mode, on the other hand, places priority on meeting the target objective at the risk of not meeting the OAR constraints. ${ }^{53}$ Typically, optimization is set to "constrained" mode, and the OAR constraints are relaxed accordingly to produce a reasonable target dose coverage.

\subsubsection{Optimization process}

As briefly described in Section 1.5, optimization within the Monaco TPS follows a threestep process: (1) generating the ideal photon fluences, (2) converting the photon fluences into deliverable beam apertures or segments, and (3) segment refinement through SSO. Depending on the technique, different combinations of these three steps can be implemented. To generate beam segments, VMAT optimization follows steps 1 and 2, while mDCAT optimization, on the other hand, only goes through step 2 because it directly generates beam apertures by conforming the MLC leaves to the target (with a user-defined margin). Regardless of the technique, SSO is always optional, and it is implemented the same way within Monaco. The optimization in the context of VMAT is described below because it involves the three steps. Some specifics about mDCAT optimization are mentioned in Chapter 3.

Before the start of an optimization, the calculation volume around all defined structures is created, and the structure volumes are calculated using cubic voxels (which are three-dimensional representations of a grid). The target volume is projected on the Beam's Eye View (BEV) with a pre-defined target-to-MLC margin. Beamlets are created with one of the two dimensions of their cross section automatically determined by the MLC leaf width. The other dimension of the beamlets cross section is configured by the user in the TPS at the start of the optimization. For the Elekta Agility MLC 
system, each MLC is $5 \mathrm{~mm}$ projected at the isocentre. The typical beamlet width defined by the user is $3 \mathrm{~mm}$. Hence, the typical dimensions of beamlet cross sections are $5 \times 3 \mathrm{~mm}^{2}$.

During step 1 of VMAT plan optimization, the ideal photon fluence reaching the patient for all beamlets are produced. The term ideal refers to the photon fluence that might be delivered when ignoring the treatment machine physical range of dynamic parameters (maximum MLC speed, minimum dose rate, etc.). The optimization continues until all the objectives and constraints are met or when it hits a pre-determined threshold, an example is when the constraint is identified as infeasible after it cannot be further maximized/minimized. After the system finds the solution of the optimization, the optimizer proceeds to step 2 of the optimization in which the ideal photon fluences are converted into deliverable beam segments, which typically degrades the ideal plan quality from the step 1 of optimization due to the physical LINAC component limitations. The limitations on the LINAC dynamic parameters are shown in Figure 2.2.

If $\mathrm{SSO}$ is selected, the optimizer proceeds to step 3 , in which the generated beam segments undergo refinements that include fluence smoothing, aperture sequencing or clustering, and optimization of beam weights and shapes. A plan undergoes five cycles of SSO, which are then followed by the final dose calculations. An example of a photon fluence for a beam segment in a VMAT plan has been shown earlier in Figure 1.8. The concept of SSO is one of the main key features of mDCAT (versus cDCAT), and it is heavily explored later in this study. 


\begin{tabular}{|c|c|c|c|}
\hline \multicolumn{3}{|c|}{ Installation: Clinic 1} & \\
\hline \multicolumn{2}{|r|}{ Clinic: } & 1 The Ottawa Hospital & \\
\hline \multicolumn{4}{|c|}{ Machine: 00 Mon5mmMLC6x } \\
\hline Default? & \multicolumn{2}{|l|}{ Parameter } & Value \\
\hline$\sqrt{\square}$ & \multicolumn{2}{|c|}{ Max. Leaf Travel Per Second (mm) } & 32.0 \\
\hline$\Gamma$ & \multicolumn{2}{|c|}{ Max. TJaw Travel Per Second (mm) } & 60.0 \\
\hline$\sqrt{\square}$ & \multicolumn{2}{|c|}{ Max. PJaw Travel Per Second (mm) } & 30.0 \\
\hline$\Gamma$ & \multicolumn{2}{|c|}{ Max. Gantry Travel Per Second (d... } & 5.6 \\
\hline$\sqrt{v}$ & \multicolumn{2}{|c|}{ Max. Collimator Travel Per Second... } & 0.0 \\
\hline$\Gamma$ & \multicolumn{2}{|c|}{ Min. Dose Rate (MU/min) } & 75.00 \\
\hline$\Gamma$ & \multicolumn{2}{|c|}{ Max. Dose Rate (MUjimin) } & 600.00 \\
\hline$\sqrt{\square}$ & \multicolumn{2}{|c|}{ Continuous Dose Rate } & $\sqrt{\square}$ \\
\hline$\sqrt{\square}$ & \multicolumn{2}{|c|}{ Beam Startup Time (sec) } & 1.00 \\
\hline$\Gamma$ & \multicolumn{2}{|c|}{ Min. MU Per Static CP } & 4.00 \\
\hline$\sqrt{v}$ & \multicolumn{2}{|c|}{ Min. MU Per Dynamic CP } & 1.00 \\
\hline$\sqrt{v}$ & \multicolumn{2}{|c|}{ Min. Gantry Deg Per CP (deg) } & 1.5 \\
\hline$\sqrt{\square}$ & \multicolumn{2}{|c|}{ Open Guard Leaves } & $\sqrt{\checkmark}$ \\
\hline$\sqrt{\square}$ & \multicolumn{2}{|c|}{ Max. Leaf Travel Per Gantry Rotat... } & \\
\hline
\end{tabular}

Figure 2.2: Physical machine limitations on the dynamic parameters for the Elekta Synergy/Infinity with the Agility MLC system. These limits are used during step 2 of the optimization, in which the ideal photon fluences are converted into beam apertures. In Chapter 7, these limits are used to investigate plan robustness.

\subsubsection{Structure layering}

The layering of the structures (target volumes and OARs) determines how the optimizer treats the voxels in the volume where the structures overlap. The structure that is listed higher in the layering order "owns" the voxels in the area of overlap with another structure, even if there is no cost function (objective or constraint) assigned to it. However, the layering of the structures does not translate into the importance or weight of the cost functions for that structure. An example is the layering of the ITV and the PTV as shown in Figure 2.3. Optimizing the ITV and PTV separately requires that the ITV be higher in layering than the PTV since all parts of the ITV are within the PTV by definition. 


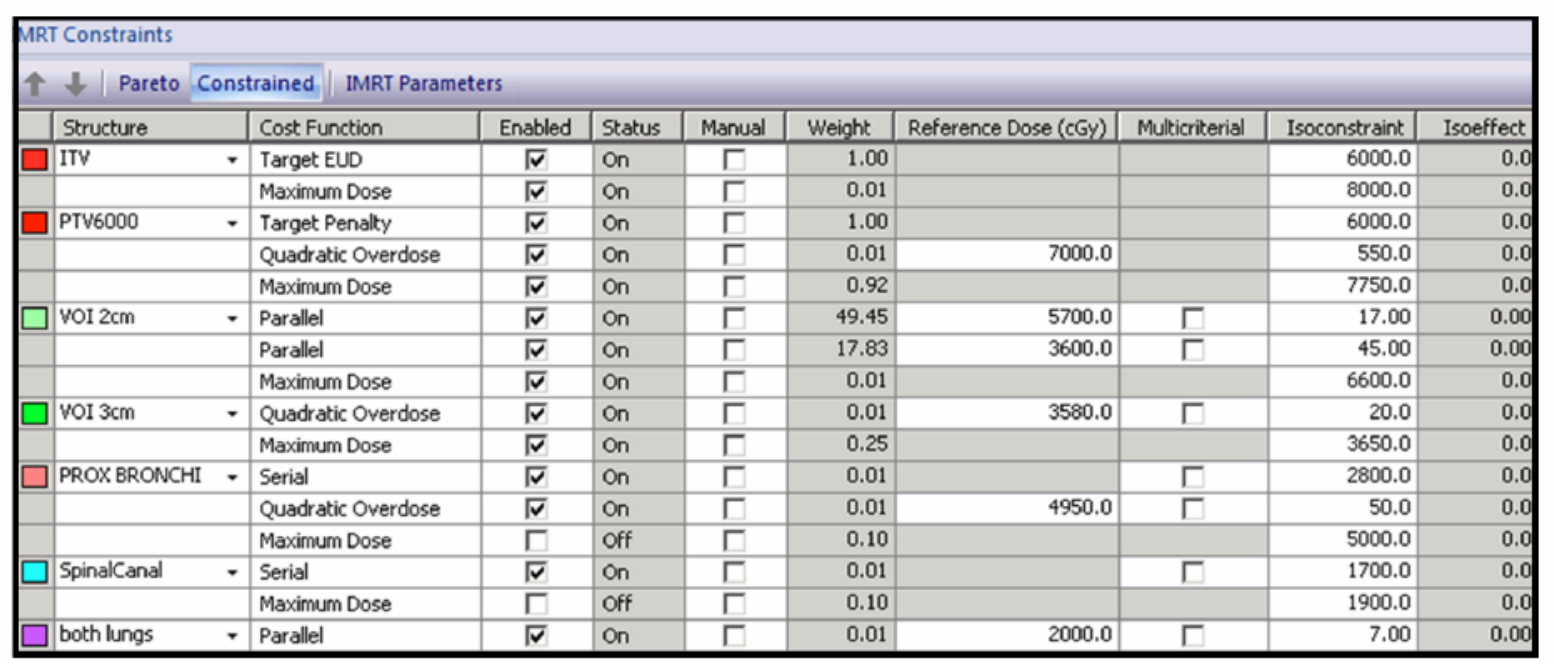

Figure 2.3: Screenshot of a set of objectives and constraints showing the layering of different targets and structures.

\subsubsection{Objectives and constraints}

Objectives are cost functions that are used to maximize the dose to the target volume, while constraints are cost functions that are used to limit the dose to the OARs. In the Monaco TPS, physical and biological objectives and constraints can be used. When using the physical objectives and constraints, the beamlet intensities within a photon fluence map are weighted to achieve the desired dose distributions. ${ }^{54,55}$ On the other hand, when using biological objectives and constraints, the beamlet intensities are weighted via radiobiological models such that the calculated dose distributions yield a known tissue response. ${ }^{56-59}$

\section{Physical objectives}

Target penalty. This objective function is used to tell the optimizer the minimum dose that a minimum volume of a structure (e.g., target) should receive. A quadratic penalty to the cost function is applied from the threshold dose down (see Figure 2.4a). 

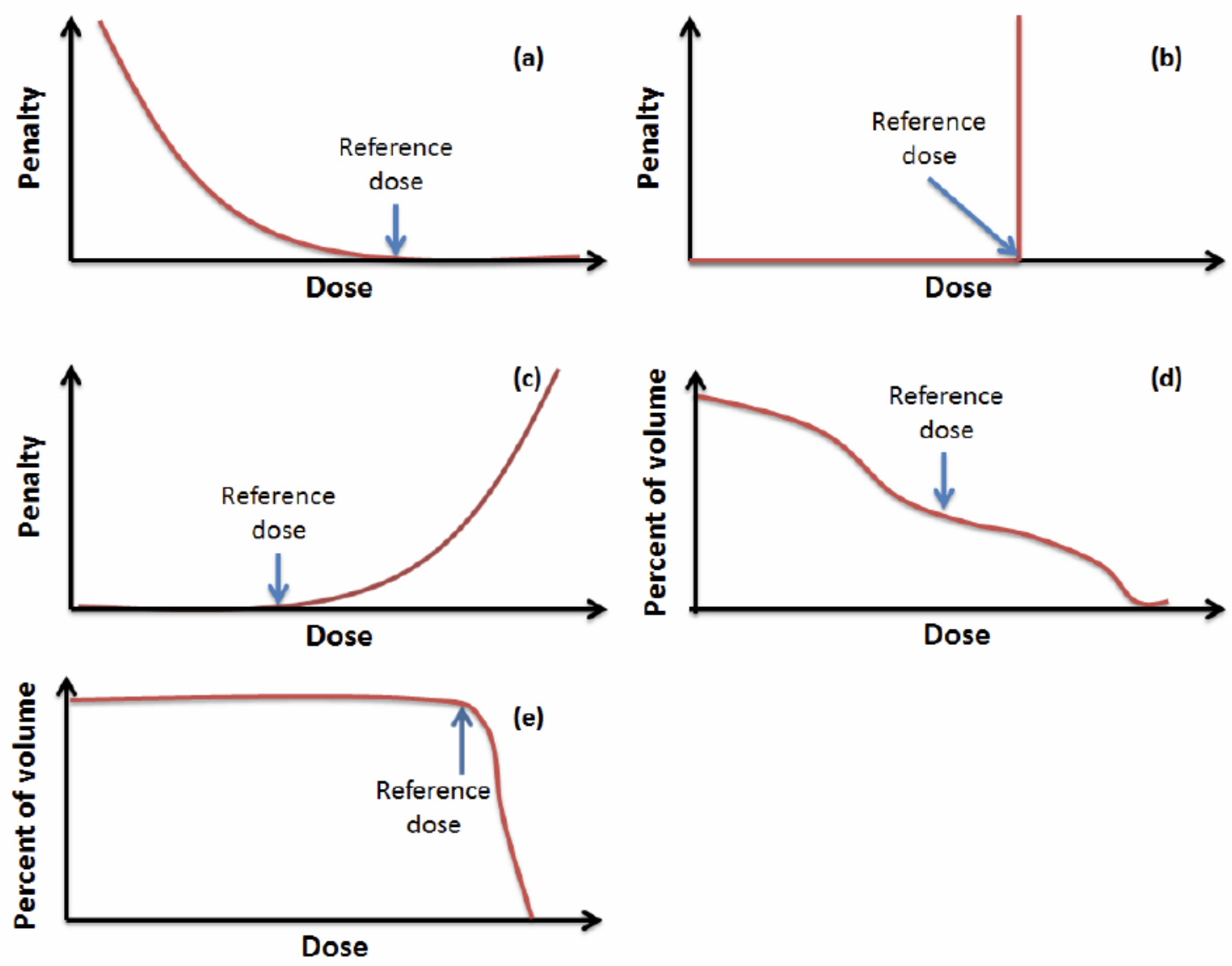

Figure 2.4: Schematics of how different physical cost functions control the DVH - (a) target penalty, (b) maximum dose, (c) quadratic overdose, (d) overdose DVH, and (e) underdose DVH. The red curve represents the DVH for the target or the OAR, and the arrow shows the relevant dose points that the optimizer uses for decision making.

\section{Physical constraints}

Maximum dose. This is a hard constraint used with either targets or OARs. The optimizer meets this criterion, even at the expense of the target coverage or OAR sparing (see Figure 2.4b).

Quadratic overdose. This constraint is used with either the target or the OAR volumes to limit high doses in the structure to which it is applied while allowing a small, yet 
controlled hotspot, penalizing the cost function quadratically (see Figure 2.4c).

Overdose DVH. This physical cost function is the equivalent of a DVH constraint for an OAR. This constraint is used to limit a certain volume of an OAR to a certain dose level. It only controls one point on the DVH. (see Figure 2.4d).

Underdose DVH. This physical cost function is used to keep a certain volume of the target to receive at least a certain dose level. Similar to overdose DVH, it only controls one point on the DVH (see Figure 2.4e).

\section{Biological Objectives}

Target EUD. The equivalent uniform dose (EUD) objective uses radiobiological models to generate heterogeneous dose distributions that produce the same biological effect as that of a given homogeneous distribution..$^{59-61}$ The required input for this objective is the cell sensitivity. For a higher cell sensitivity, the penalty paid is increased for cold spots within the selected structure.

\section{Biological Constraints}

Serial constraint. The serial cost function is the biological equivalent of a maximum dose penalty, in which high doses are harmful even if they are limited to small volume of the structure. The required parameters are EUD and power law exponent. The EUD is the dose that causes the same damage if applied uniformly to the entire structure. The power law exponent $k$ is a volume-effect parameter. With higher $k$ values, there is less tolerance for excessive damage to small volume of the structure, giving more weight to high dose volumes. On the other hand, lower $k$ values give comparable weighting to low dose and high dose volumes (see Figure 2.5). Examples of serial OARs where the 


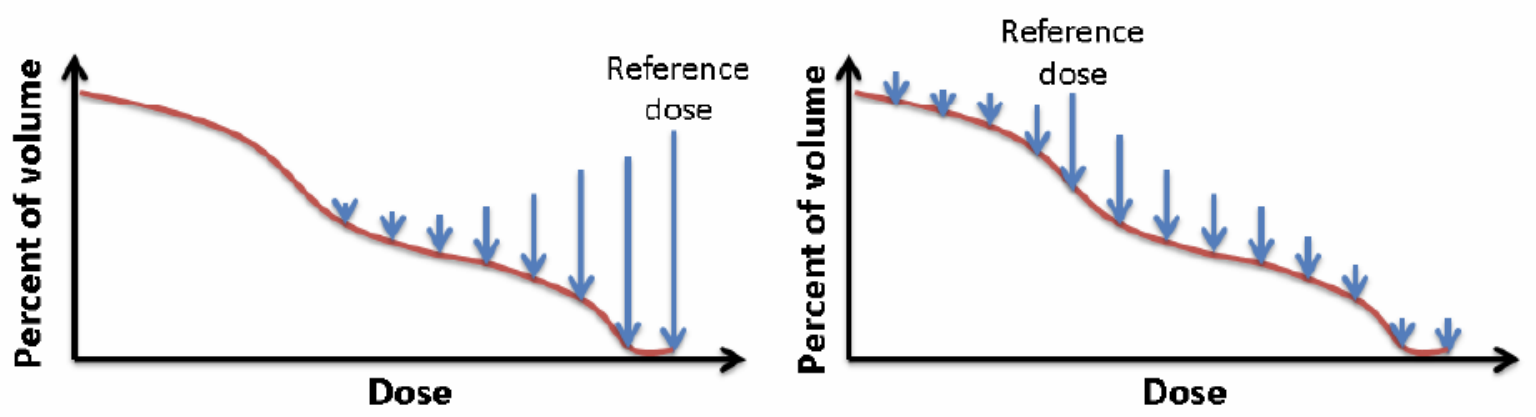

Figure 2.5: Schematics of how serial (left) and parallel (right) biological constraints control the DVH. The red curve represents the DVH for the OAR, and the arrow length is proportional to how hard the optimizer works at that dose-volume level.

Serial constraint is typically useful are spine, esophagus and optic structures.

Parallel constraint. The parallel cost function allows higher doses in small volumes of the structure, if the rest of the structure is spared. ${ }^{62}$ The required parameters are reference dose, mean organ damage and power law exponent. The reference dose is the dose acceptable to the majority of the structure, and the mean organ damage is the tolerance percent volume of the structure. The power law exponent $k$ changes the shape of the dose response of the structure, and higher $k$ values imply steeper dose response, producing a kink at the intersection point of the mean organ damage and the reference dose on the DVH curve (see Figure 2.5). Examples of OARs that have parallel functional sub-units where the Parallel constraint is typically useful are lungs, kidneys and liver.

\subsubsection{Avoidance Structure}

An organ can be blocked from any angle of the arc if it is set as an avoidance structure. Typically, heavily-modulated treatments such as VMAT do not require the use of this feature since the modulation itself can spare the overlapping or the adjacent OARs. This feature is available in mDCAT, and is explored in Chapter 3. 


\subsubsection{Beam sequencing parameters}

For each treatment technique, there are sequencing parameters that can be configured by the user prior to the start of optimization. The optimizer respects the minimum and/or maximum limit values defined in the sequencing parameters when generating beam segments. Each treatment delivery technique has its own set of sequencing parameters (see Figure 2.6). Discussed below are the relevant sequencing parameters for VMAT and mDCAT.

Segment Shape Optimization. SSO, as mentioned earlier, refines the initial beam segments generated during step 2 of the optimization. This option is available for both VMAT and mDCAT planning, and is explored for mDCAT later in this study.

Maximum Number of Arcs. In VMAT, for each arc range defined by a beam, this parameter tells the system the maximum number of rotations within that arc range. This option is not available with mDCAT.

Maximum Number of Control Points Per Arc. Control points are the starting and ending positions of the LINAC components for each beam segment during treatment delivery. The control point that defines the end of a beam segment is the same control point that defines the beginning of the following beam segment. Defining the maximum number of control points (and correspondingly the number of beam segments) is only meaningful for VMAT optimization because of the ability to modulate over the target, thus producing any number of beam segments in the final treatment plan. On the other hand, mDCAT is controlled by the fact that the starting point for segment shapes is 


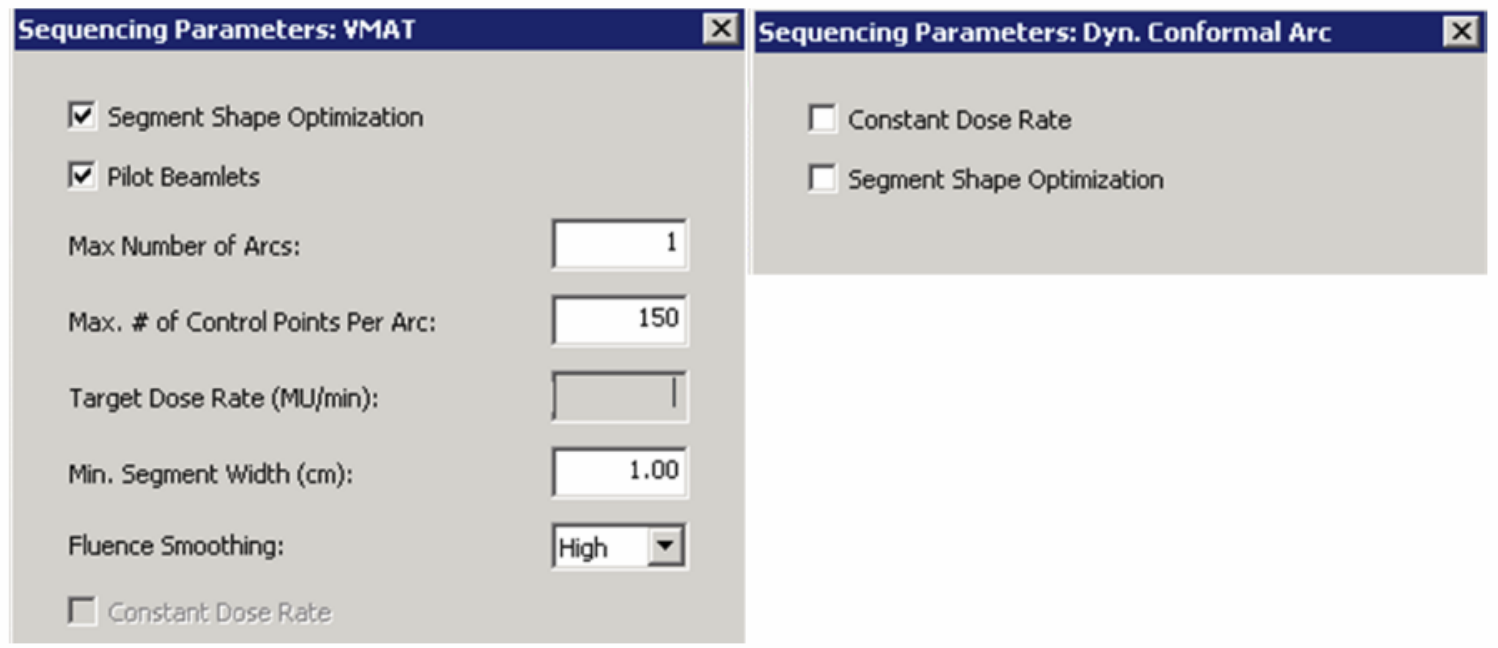

Figure 2.6: Sequencing parameters for VMAT (left) and mDCAT (right) in the Monaco TPS.

by conforming to the target boundaries, and thus the number of segments is fixed.

Target Dose Rate. This feature is only available when SSO is not selected in VMAT plans. If SSO is selected, the dose rate for each control point is chosen by the optimizer. This feature is not available for mDCAT. The dose rate in mDCAT can only be set to either constant or variable, which is further discussed in Chapter 3.

Minimum Segment Width. This is defined as the minimum distance between two opposing MLC leaves in a beam segment. This feature is only available for VMAT.

Fluence Smoothing. This parameter controls the smoothing of the photon fluence generated during the step 1 of the optimization for VMAT. For low smoothing value, a higher number of segments can be generated and there are steeper gradients within the photon fluence between neighbouring beamlets. For high smoothing value, the photon fluence generated is smoother. However, choosing high smoothing can degrade the plan by not allowing necessary steep gradients. In this study, medium smoothing was used in the treatment planning for different disease sites, while the high smoothing was used for 
lung SBRT cases to minimize the interplay between the plan delivery and the tumour motion due to breathing (Chapter 7).

\section{$2.3 \quad$ Radiation beam model}

To accurately simulate the LINAC geometries during treatment planning, the radiation beam model is commissioned in the TPS prior to clinical use. The beam model in the Monaco TPS uses a virtual source model which consists of probability distributions that describe a primary photon source, a scattered photon source, and an electron contamination source. These probability distributions are informed by modeling using Monte Carlo simulations research in LINAC beam. ${ }^{63} \mathrm{~A}$ virtual source model treats each part of the LINAC as a radiation source, replacing the need for a full Monte Carlo simulation of the LINAC head to generate radiation source particles. During TPS commissioning, LINAC measurements are compared to the dose distributions generated by the TPS using the LINAC beam model, and the beam model is refined until a clinically-acceptable level of agreement is reached between the measured and calculated values. $^{64}$

\subsection{Dose calculation algorithms}

Dose calculations in the TPS require the radiation properties of different patient tissues. The Monaco TPS has built-in mathematical functions that represent the radiation properties (photon attenuation coefficients, electron stopping powers, etc.) based on electron densities. These functions assume standard densities and compositions of the

human tissues. The electron densities are extracted from the HU-to-ED conversion curve that is used during the patient simulation stage of the EBRT process described 
earlier in Section 1.1. In the Monaco TPS, the dose deposited to the tissues can be calculated using one of three options: (1) radiation transport in medium and score in medium, (2) radiation transport in medium and score in a small mass of water at the scoring voxel, and, (3) radiation transport in water and score in water. Options 2 and 3 have their relevance in certain dosimetry studies and in dose calculations in the presence of severe artifacts. The default is to transport radiation in medium and score in medium, and this is used in the dose calculations in this study.

There are three dose calculation algorithms available in the Monaco TPS: Monte Carlo, finite size Pencil Beam and collapsed cone. Depending on the treatment technique, a dose calculation algorithm is preferred taking into consideration the required calculation accuracy and planning efficiency. These three calculation algorithms are used for the dose calculations in this study.

Monte Carlo. Monte Carlo is a general numerical approach that uses random sampling and known probability density functions of radiation interactions to model radiation transport in media and in patient tissues. A large number of individual radiation tracks (or histories) are simulated to produce the dose distributions in the patient. To date, Monte Carlo algorithm has been shown to be the most accurate approach in dose calculation for treatment areas with high tissue heterogeneities, and it has been used to benchmark other dose calculation algorithms. ${ }^{65}$ However, compared to other calculation algorithms, Monte Carlo is less efficient and the calculation time can vary drastically depending on the desired level of statistical uncertainty on the calculated dose distributions. For example, to reduce the statistical uncertainty by a factor of two, the number of particle histories should be increased by a factor of four, which consequently increases the calculation time by four. In the Monaco TPS, the statistical uncertainty for the 
Monte Carlo algorithm can be specified as either per plan or per control point. For the statistical uncertainty per plan, the TPS calculates the uncertainty in the dose to the central voxels based on the contributions from all segments in the plan, and uses this information to monitor the number of histories to be simulated until the desired overall uncertainty is reached. For the statistical uncertainty per control point, the same approach is used but on a segment-by-segment basis, which means that more histories are needed for the same uncertainty level per control point than per plan. In this study, Monte Carlo was used for dose calculations to accurately handle tissue heterogeneities, and a $1 \%$ per plan was used for the statistical uncertainty.

Finite size Pencil Beam. Finite size Pencil Beam dose calculation algorithm assumes that large radiation beams can be represented as the sum of identical finite size pencil beams. The shape of the pencil beam changes according to the traversed tissue density. The dose distribution created by the beamlets are calculated by multiplying the $2 \mathrm{D}$ formula of the pencil beam to a scaling factor which is dependent on the depth and offset of the beamlet from the central axis of the beam. ${ }^{66,67}$ This scaling factor accounts for the changes in ranges and lateral spread of scatter electrons and photons. The density corrections were verified through Monte Carlo simulations.

Collapsed cone. With the collapsed cone algorithm, the photon beam coming out of the gantry head is ray-traced through the patient, and the distribution of the total radiant energy released into the patient is calculated. Polyenergetic energy deposition kernels are calculated from the spectrum of the beam, using a database of pre-calculated monoenergetic kernels (energy deposition kernels are dose distributions by the charged particles set in motion around a photon interaction). The convolution of these polyenergetic kernels yields dose distributions facilitated by the introduction of the collapsed 
cone approximation. In this approximation, all energy released into coaxial cones of equal solid angle from volume elements on the cone axis is rectilinearly transported, attenuated, and deposited in elements "on the axis". Scaling of the kernels is implicitly done during the convolution procedure to account for inhomogeneities present in the irradiated volume. When compared to the Monte Carlo algorithm, general agreement is excellent except for some deviations observed in situations of lateral charged particle disequilibrium in low-density media. ${ }^{68}$ In the Monaco TPS, collapsed cone is only available for 3DCRT planning.

Robustness of the VMAT and mDCAT plans against machine and patient uncertainties are evaluated in Chapters 6 and 7, respectively. Robustness was assessed in this study by quantifying the dosimetric impact of small, realistic perturbations to the machine/patient geometry relative to the case without perturbation. The number of these calculations is very large, and the differences due to the perturbations can be small. To achieve very small statistical uncertainty on Monte Carlo that would be smaller than some of the effects being investigated would take prohibitively long time on clinical systems, and this is why finite size Pencil Beam was used for these robustness calculations only (the rest of the calculations in this study are done with Monte Carlo). The accuracy of the dose calculation algorithm largely cancels out when what's of interest is only the perturbation effect. Tables 2.1 and 2.2 demonstrate a comparison of the results for a typical robustness test, in which the impact on some of the standard plan quality metrics was evaluated. These metrics are discussed further in Chapter 5.

In summary for this study, Monte Carlo with $1 \%$ statistical uncertainty per plan was used for the dose calculations of VMAT and mDCAT plans, except for the robustness analysis, in which finite size Pencil Beam was used. Collapsed cone was used for the dose calculations of 3DCRT plans. 
Table 2.1: An example to demonstrate the impact of the choice of the dose calculation algorithm on the accuracy of estimating perturbation effects. The perturbation effect in this example is artificially moving the MLC leaves by $2 \mathrm{~mm}$ from their true positions. Results are for mDCAT and VMAT plans of a palliative spine patient. $\mathrm{MC}$ refers to Monte Carlo (using $0.1 \%$ uncertainty per control point), and PB refers to finite size Pencil Beam. Dx\% is the dose received by $\mathrm{x} \%$ of the volume of the structure. The data demonstrates that the difference between $M C$ and $P B$ in quantifying the effect of a perturbation during robustness calculations is much smaller than the perturbation effect itself.

\begin{tabular}{|c|c|c|c|c|c|c|c|c|}
\hline \multirow{3}{*}{ Technique } & \multicolumn{8}{|c|}{ Dosimetric impact in percent } \\
\hline & \multicolumn{2}{|c|}{$\begin{array}{c}\text { PTV } \\
\text { vertebrae } \\
\mathrm{D}_{95 \%}\end{array}$} & \multicolumn{2}{|c|}{$\begin{array}{c}\text { OAR } \\
\text { spinal canal } \\
\mathrm{D}_{2 \%}\end{array}$} & \multicolumn{2}{|c|}{$\begin{array}{c}\text { OAR } \\
\text { left kidney } \\
\text { mean dose }\end{array}$} & \multicolumn{2}{|c|}{$\begin{array}{c}\text { OAR } \\
\text { right kidney } \\
\text { mean dose }\end{array}$} \\
\hline & PB & $\mathrm{MC}$ & PB & $\mathrm{MC}$ & PB & $\mathrm{MC}$ & PB & $\mathrm{MC}$ \\
\hline mDCAT & 0.9 & 0.7 & 1.1 & 0.8 & 3.4 & 3.5 & 3.2 & 3.1 \\
\hline VMAT & 3.8 & 3.7 & 4.7 & 4.8 & 3.1 & 3.1 & 3.9 & 3.9 \\
\hline
\end{tabular}

Table 2.2: Same as in Table 2.1, but for a representative lung SBRT patient. The additional metric $\mathrm{V}_{20 G y}$ is the fractional volume of combined healthy lungs receiving 20 Gy.

\begin{tabular}{|l|l|l|l|l|l|l|}
\hline \multirow{3}{*}{ Technique } & \multicolumn{5}{|c|}{ Dosimetric impact in percent } \\
\cline { 2 - 7 } & $\begin{array}{c}\text { PTV } \\
\text { lung tumour } \\
\mathrm{D}_{95 \%}\end{array}$ & $\begin{array}{c}\text { OAR } \\
\text { spinal canal } \\
\mathrm{D}_{2 \%}\end{array}$ & \multicolumn{2}{c|}{$\begin{array}{c}\text { OAR } \\
\text { combined healthy lungs } \\
\mathrm{V}_{20 G y}\end{array}$} \\
\cline { 2 - 7 } & $\mathrm{PB}$ & $\mathrm{MC}$ & $\mathrm{PB}$ & $\mathrm{MC}$ & $\mathrm{PB}$ & $\mathrm{MC}$ \\
\hline mDCAT & 2.3 & 3.1 & 1.8 & 1.8 & 1.4 & 1.4 \\
VMAT & 9.5 & 8.6 & 2.5 & 2.4 & 1.2 & 1.2 \\
\hline
\end{tabular}

\subsection{Patient selection}

mDCAT is likely to be less demanding on the machine during treatment delivery because it does not incorporate the high degree of modulation that VMAT has. This can be of advantage in terms of plan optimization and treatment delivery efficiency, and robustness against different uncertainties, and these aspects are explored later in this study. These possible advantages are the main reasons for choosing spine, lung SBRT 
and brain as test cases in this study. In the sub-sections below, the rationale for choosing these disease sites is further explained, the anonymized patients included in this study are listed, and the range of target locations and volumes is described.

\subsubsection{Spine metastases}

The majority of palliative RT is for pain control from spine metastases. ${ }^{69,70}$ Ideally, treatment of painful skeletal metastases should be expedited to offer prompt symptom relief. For palliative RT, the conventional preparation and delivery process can be impractical because it involves hours of waiting and the whole process of CT simulation, treatment planning, plan verification, and treatment delivery. Currently, dedicated solutions for rapid palliation for spine metastases such as treatment planning and dose calculations on pre-treatment CBCT using the simplest treatment techniques (e.g., 3DCRT with single or parallel-opposed beams) have the promise of doing the entire EBRT process in a typical treatment timeslot of 30 to 60 minutes. ${ }^{71-75}$ However, this kind of non-conformal treatment typically exposes the OARs to higher doses, which can have early and late side effects that goes against the drive to improve the quality of life for palliative patients. It also leaves less dosimetric room for future treatments that involve the same OARs (palliative cancer patients currently live longer because of the overall improvements in cancer detection, treatment and management). Using VMAT typically reduces the doses to the OARs, but the optimization process (optimizing the photon fluences, generating the beam segments, and refining the segments), plus the Monte Carlo dose calculation efficiency become a bottleneck given the very limited timeline. Overall, mDCAT may have the potential to offer conformal plans that have better quality than the simple treatment techniques, but are less complicated to

implement. Moreover, because of the relatively less complexity of mDCAT deliveries 
compared to VMAT, patient-specific QA requirements are less stringent and treatment planning while the patient is on the treatment couch can be more feasible. Because of all these considerations, palliative spine cases were included in this study to assess plan optimization and treatment delivery efficiency, as well as plan quality.

The spine has four major sections, namely, the cervical, thoracic, lumbar and sacral. The surrounding OARs vary depending on the location of the spine tumour. The cervical spine consists of seven vertebrae starting superiorly from the base of the skull down to the level of the clavicular notch. The most common surrounding OARs are the oral cavity, esophagus, and larynx. The thoracic spine is the longest section of the spine and consists of twelve vertebrae starting superiorly at the clavicular notch down to the middle of the abdomen. The upper portion of the thoracic spine is surrounded by the lungs, heart, breasts or chestwalls, middle esophagus, and liver. Down the lower thoracic spine are the liver, stomach, bowel, and sometimes the upper portion of the kidneys. The lumbar spine has five vertebrae surrounded mainly by the kidneys and the bowel. The sacral spine is located mainly in the pelvic region and has five vertebrae surrounded by the bladder, rectum, and femurs.

In this study, twenty five representative palliative spine cases were chosen. These patients were treated at TOHCC for palliation between 2013 and 2016. The patients were treated with either 3DCRT or VMAT. The PTVs are located at different sections of the spine (see Table 2.3), with one to eight vertebrae included in the PTV, with volumes ranging from 150 to $1242 \mathrm{~cm}^{3}$. The Appendix contains the full list of the anonymized twenty five palliative spine patients included in this study. 
Table 2.3: Target sizes (in number of vertebrae) and locations for the twenty five palliative spine patients included in this study.

\begin{tabular}{|r|l|}
\hline Spine section & Number of vertebrae in the PTV (number of patients) \\
\hline Cervical & $3(1), 4(1)$ \\
Cervical and thoracic & $6(2)$ \\
Thoracic & $1(2), 2(1), 3(2) 4(3), 5(1), 6(1), 8(1), 9(1)$ \\
Thoracic and lumbar & $4(1), 6(1)$ \\
Lumbar & $2(1), 3(1), 4(1)$ \\
Lumbar and sacral & $2(1), 4(1), 8(1)$ \\
Sacrum & $4(1)$ \\
\hline
\end{tabular}

\subsubsection{Lung SBRT}

Motion due to breathing is the most common example of patient uncertainties during treatment. If not accounted for properly, this can cause underdosage to the target and overdosage to the surrounding healthy tissues. ${ }^{76}$ Lung SBRT cases have small targets which are more vulnerable to both patient and machine uncertainties. SBRT treatments follow hypofractionation in which a certain error in one fraction has a larger impact on the total dose delivered to the patient (compared to conventionally-fractionated treatments with many more fractions and a smaller dose per fraction, where the cumulative effect of random daily errors is more likely to wash out). The use of VMAT for lung SBRT has the potential to cause large modulation on such a small field. In this study, lung SBRT cases were used to assess plan quality, and to investigate the effect of machine and patient uncertainties on plan robustness when using mDCAT versus VMAT.

Twelve representative lung SBRT patients treated with VMAT between 2015 and 2016 at TOHCC were chosen. Seven patients had tumour on the right lung and five patients had tumour on the left lung. The PTV sizes ranged from 7 to $51 \mathrm{~cm}^{3}$. Five 
of the patients had the PTV attached to the inner chest wall, and the remaining seven had the PTV in the middle of the lung. This variety of PTV locations should show the plan quality achievable for different surrounding OARs, and the plan robustness for targets in locations with different motion characteristics. The Appendix has the full anonymized list of the lung SBRT patients included in this study.

\subsubsection{Brain}

cDCAT has been shown to be effective for small brain tumors, such as in brain SRS (see Section 1.7). Circular fields are typically used to treat these types of targets. For a target with diameter larger than that of the circular field, the dose is "painted" over the target. Exceeding the tolerance values of the OARs in the vicinity of the brain can compromise the quality of life of the patient (e.g., can cause blindness if the dose to the optic structures exceeds the tolerance values). The use of VMAT for small brain tumours can cause a high degree of modulation, which may lead to higher dosimetric impact in the presence of machine uncertainties. Small tumours with hypofractionated treatments (such as lung SBRT and brain cases included in this study) are typically treated on LINACs with stereotactic cones or on specialized machines such as Cyberknife (see Section 1.5). Therefore, mDCAT may be more appropriate for treating such targets given that mDCAT is much closer to how such targets have been traditionally treated with successful results than VMAT which represents a larger

deviation from recent practice due to the higher degree of modulation over such small targets.

Brain cases were included in this study to compare the plan quality achievable with mDCAT versus that with cDCAT and VMAT. The brain cases were also used to compare the robustness of mDCAT and VMAT against machine uncertainties (Chapter 6). 
Twelve brain patients treated on CyberKnife between 2014 and 2016 at the TOHCC were chosen. All cases had a single target, with volumes ranging from 2 to $20 \mathrm{~cm}^{3}$. Four of the brain patients had the most critical OARs adjacent to or overlapping with the PTV. By including these complex cases in the set of brain patients included in this study, the capabilities of mDCAT in sparing very close OARs can be explored. The Appendix contains the full anonymized list of the twelve brain patients included in this study.

\subsection{Treatment planning methodologies}

Described below are the specific treatment planning settings used for the 3DCRT, VMAT and mDCAT in this study. Spine cases were planned with 3DCRT, VMAT and mDCAT. For the lung SBRT and brain cases, 3DCRT was not investigated since currently it is not used for stereotactic treatments in general. Plan quality was assessed using the metrics described in Section 2.7 - namely 3D dose distributions, DVHs for the targets and OARs, and three single-value plan quality metrics: Conformity Index (CI), Homogeneity Index (HI), and Gradient Index (GI)

3DCRT planning. For the 3DCRT plans created for the palliative spine patients included in this study, coplanar (i.e., with a zero couch angle) parallel-opposed beam arrangement was chosen, with one beam coming from the anterior and the other beam coming from the posterior direction. An exception was with the cervical spine patients, in which lateral parallel-opposed beams were used in order to reduce the dose to the oral cavity. These beam arrangements are the ones typically used for rapid-access palliation. The MLC leaves were shaped around the target volume with a target-to-MLC margin between 7 to $8 \mathrm{~mm}$. For 3DCRT with coplanar beams, it is known that the superior 
and inferior edges of the PTV receive less radiation dose than other parts of the PTV because there are no beams entering or exiting through the superior and inferior PTV edges, and hence there is lack of volume scatter from outside the beam at those two edges (compared to other points of the PTV that are not on the superior and inferior edges). ${ }^{51}$ A common approach ${ }^{51}$ used during 3 DCRT planning to circumvent this issue is to manually open the field borders slightly more in the superior and inferior directions than in the other directions to allow for full dose coverage at those edges with coplanar beams. For the cases that exceeded the $107 \%$ recommended maximum dose with only open fields, subfields were created as discussed earlier in Section 1.5.1. The energies used were $6 \mathrm{MV}, 10 \mathrm{MV}$, or a combination of both, depending on the size of the patient and the depth of the tumour.

VMAT planning. For the cases that had existing VMAT plans that were used clinically to treat the selected patients, these plans were used as-is in this study. For the cases that did not have clinical VMAT plans, an experienced treatment planner created VMAT plans that met high quality clinical standards. For the spine cases, a full rotation coplanar arc with $6 \mathrm{MV}$ photon beam energy and a $3 \mathrm{~mm}$ dose calculation grid spacing were used. For the lung patients, partial arcs were used, which avoided the beam entry at the healthy contralateral lung. For brain cases, multiple non-coplanar arcs (with non-zero couch angle) were used, which is typical for intra-cranial targets. For lung and brain stereotactic-type treatments, the calculation grid spacing used was $2 \mathrm{~mm}$ instead of $3 \mathrm{~mm}$ to reduce volume averaging effects due to the small target sizes and the steep dose gradients. Moreover, for the VMAT planning of the brain cases, the minimum segment width was set to $5 \mathrm{~mm}$ instead of the conventional $10 \mathrm{~mm}$ (used for spine) because some of the brain target volumes are quite small (i.e., less than $10 \mathrm{~cm}^{3}$ ) and thus smaller beamlet widths lead to better plan quality. 
The issue of lack of volume scatter at the superior and inferior edges of the beam does not exist for VMAT, even with only coplanar deliveries because of the ability of VMAT to create random segment shapes that do not have to conform to the target shape in any direction, including the superior and inferior edges, and thus the optimizer generates some open segments beyond the superior and inferior borders to provide proper PTV coverage, without the need for user intervention.

mDCAT planning. Two approaches were used in mDCAT planning. In the first approach, the mDCAT plans were started using the objectives and constraints of the existing VMAT plan for each patient, and the resulting dose distributions were evaluated. Typically, the objectives and constraints had to be relaxed to accommodate the limited degrees of freedom that mDCAT has versus VMAT, until a clinically acceptable plan was achieved. In the second approach, mDCAT plans were created from scratch instead of relaxing the objectives and constraints of the VMAT plans. The results of this exercise showed that the typical construction of VMAT set of objectives and constraints is suitable for mDCAT planning and does not limit the potential for producing high quality mDCAT plans. The final plans were reviewed and modified if necessary by the experienced treatment planner to ensure that the plans met the clinical standards. The arc range and dose calculation grid size was kept the same between pairs of VMAT and mDCAT plans for fair comparison of plan quality. Unlike VMAT, the minimum beam segment for mDCAT cannot be configured in the Monaco TPS. The other features of mDCAT are characterized in Chapter 3, and are consequently explored to find the most appropriate beam geometries for mDCAT planning.

In this study, it was found that, for mDCAT planning with coplanar arcs, the lack of volume scatter at the superior and inferior borders of the beam exists for the same 
reason it exists for 3DCRT with coplanar beams. To circumvent this issue, an artificial PTV was created where it was extended a few $\mathrm{mm}$ in the superior and inferior directions beyond the true superior and inferior edges of the PTV. This artificial PTV was used to conform the segments to, with the user-defined margin. However, the true PTV was used for plan quality evaluation and for all the results presented in this study. The additional time overhead to create this artificial PTV for the purpose of creating the initial beam segments in mDCAT was minimal.

Plan normalization. Two types of normalization were used throughout this study. In the first type, the plans were normalized such that $95 \%$ of the PTV received the Rx, which is the accepted standard in clinical practice. This is expressed as $D_{95 \%}=R x$, and it has a unit of cGy. This type of normalization evaluates the quality of OAR sparing if the target coverage is met. In the second type, the plans were normalized such that the dose-volume constraints for the most relevant OARs were met. This type of normalization evaluates the quality of target coverage if the OAR sparing is met.

\subsection{Plan quality assessment metrics}

Standard plan quality metrics were used in this study, which include 3D dose distributions, cumulative Dose Volume Histograms (DVHs), Conformity Index (CI), Homogeneity Index (HI), Gradient Index (GI), and OAR doses. These metrics are described below.

$3 D$ dose distributions. Within the TPS, isodose lines (that join points of equal dose) are overlaid on the patient anatomy, and can be viewed on the axial, sagittal or coronal CT view. This gives visual information on how much dose a structure is receiving. The overall dose distributions within the patient is the sum of the dose from all the 


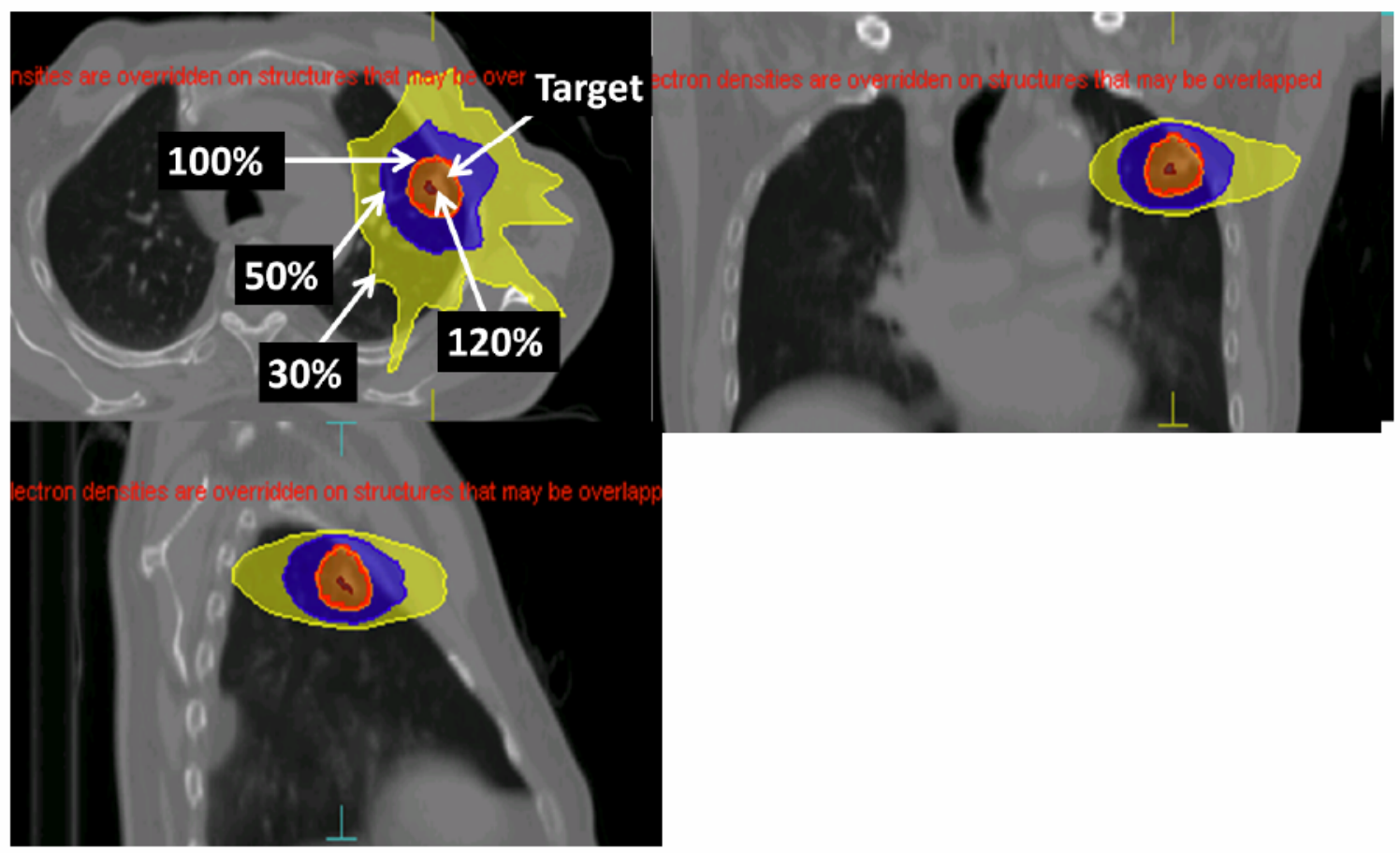

Figure 2.7: Example of the dose distributions in the axial (top left), coronal (top right) and sagittal (bottom left) views with the delineated PTV (red structure) in the Monaco TPS. The color-washes represent the following percent of the Rx: $30 \%$ (yellow), $50 \%$ (blue), 100\% (orange) and 120\% (brown).

beams and should ideally be uniform to within $95 \%$ to $107 \%$ of the prescribed dose $(\mathrm{Rx}){ }^{3,7}$ However, for cases with very small targets, the aim of treatment moves away from homogeneity within the target towards ablating the target, and higher maximum doses inside the target (e.g., 125\%) are deemed desirable. ${ }^{14,20,27,77}$ Shown in Figure 2.7 are the dose distributions for a treatment plan generated in the Monaco ${ }^{\mathrm{TM}}$ (Elekta, Stockholm, Sweden) TPS for an example patient with lung tumour.

Cumulative Dose Volume Histogram (DVH). A cumulative DVH is a graphical summary of the $3 \mathrm{D}$ dose distributions. It gives information on the amount of dose a certain volume of a structure is receiving. A differential DVH is the derivative of the cumulative DVH and it essentially provides the same information as the cumulative DVH. Shown in Figure 2.8 is a schematic for a cumulative DVH and some specific dose-volume points 


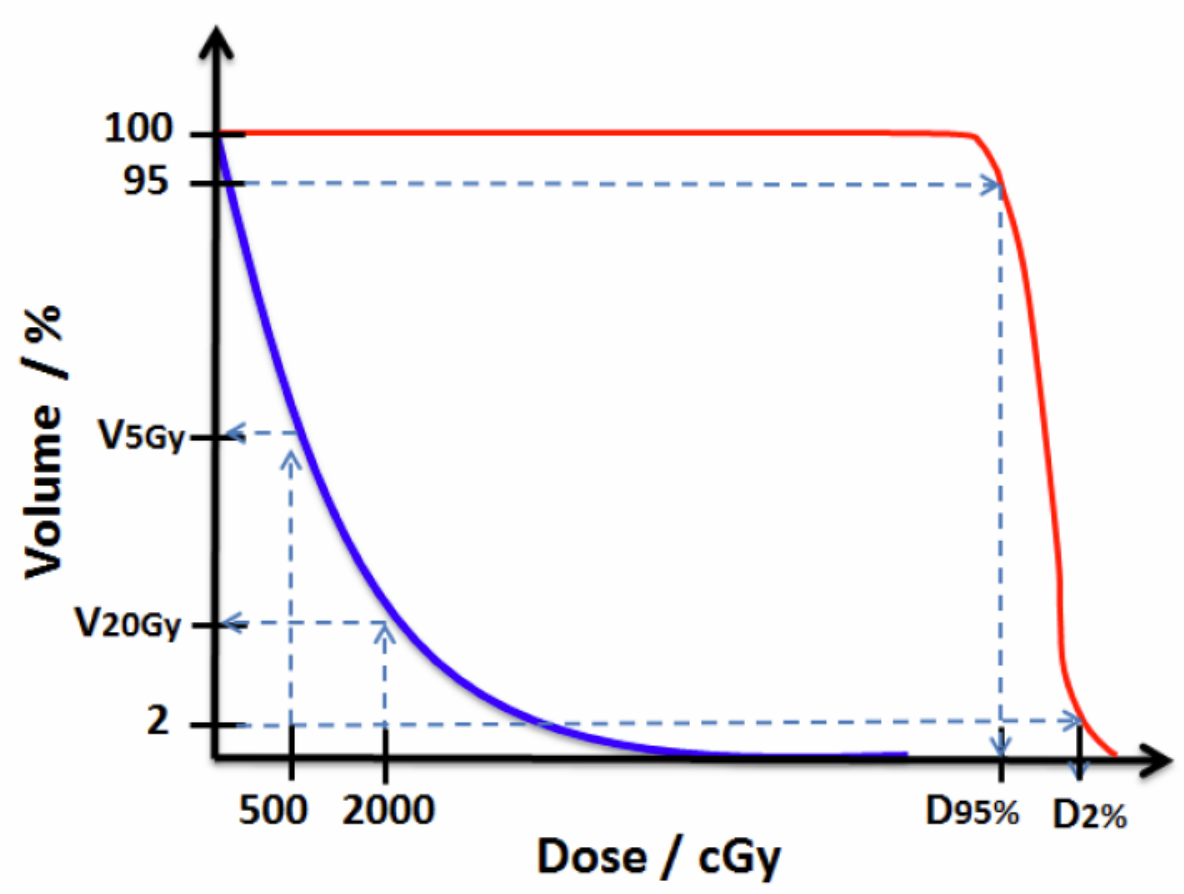

49

Figure 2.8: Schematic of the DVH dose-volume points extensively used in this study. $\mathrm{V}_{20 G y}$ and $\mathrm{V}_{5 G y}$ are the volumes receiving 2000 cGy and 500 cGy, respectively. $\mathrm{D}_{95 \%}$ and $\mathrm{D}_{2 \%}$ are the doses received by the $95 \%$ and $2 \%$ of the structure.

used in this study. $\mathrm{D}_{x \%}$ is the dose in cGy received by $\mathrm{x} \%$ volume of a structure, which is mainly used in this study to report target dose coverage and homogeneity. On the other hand, $\mathrm{V}_{x G y}$ is the volume in percent receiving $\mathrm{x}$ Gy, and it is mainly used in this study to report doses received by the OARs. Compared to full 3D dose distributions, the DVH is a more concise tool for evaluating plan quality. However, its main limitation is that it does not provide spatial information on where the different dose levels exist within a particular structure.

Conformity Index. The Conformity Index (CI) is used to characterize the degree of conformality of the $\mathrm{Rx}$ to the target volume. A definition by the $\mathrm{ICRU}^{7}$ takes the ratio of the treated volume (tissue volume enclosed by the dose of interest) to the volume of the target, with the ideal value being unity. Although this definition is easy to interpret, its major drawback is that it does not take into account the spatial intersection nor the shape of the two volumes. Partial overlap or lack of overlap between the treated and the 
(a)

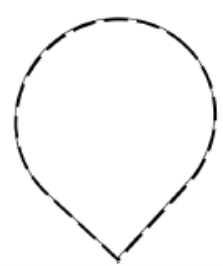

(b)

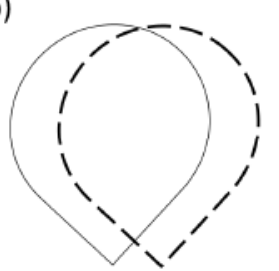

(c)

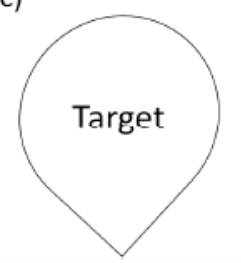

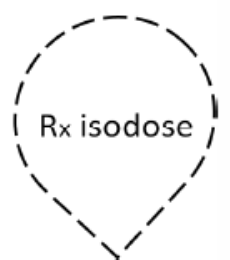

Figure 2.9: Different scenarios where the CI is calculated as the ideal value, 1, by getting the ratio of the volume of the reference isodose line with $\mathrm{Rx}$ dose to the target volume. The solid contour represents the target, while the dashed contour represents the reference dose. Only scenario (a) represents the true ideal conformality of the reference dose to the target.

target volumes can still give the ideal value of unity (see Figure 2.9). To compensate for the shortcoming of this definition, van't Riet et $a l^{78}$ proposed a definition for CI given by

$$
C I=\frac{T V_{R I}}{T V} \times \frac{T V_{R I}}{V_{R I}}
$$

where $T V_{R I}$ is the absolute volume of the target covered by the reference isodose line with $\mathrm{Rx}$ dose, $V_{R I}$ is the volume of the reference isodose line with $\mathrm{Rx}$ dose, $T V$ is the volume of the target. The first ratio quantifies the dose coverage of the target, and the second ratio quantifies the volume of healthy tissues receiving dose greater than or equal to the reference isodose. The CI value calculated using this definition ranges from 0 to 1 , with 1 being the ideal value. ${ }^{79}$ This definition for CI was used in this study.

Homogeneity Index. The Homogeneity Index (HI) is used to characterize the uniformity of the dose within the target. Different definitions of the HI have been proposed and are being used in the literature, depending on the treatment technique. For example, in radiosurgery, the HI is defined by the Radiation Therapy Oncology Group (RTOG) ${ }^{80}$ as the ratio between the maximum dose and the prescription dose $(\mathrm{Rx})$. This definition gives information on the magnitude of overdosage within the target but does not give 
information on the underdosing within the target. Wu et $a l^{81}$ defined the $\mathrm{HI}$ as the maximum minus the minimum dose, normalized to the Rx. However, the use of maximum and minimum doses is not currently recommended since they are only point doses and may not reflect the true homogeneity within the target. ${ }^{82}$ The ICRU ${ }^{82}$ suggested a definition for the HI that covers all the shortcomings of the previous definitions, and it is defined as

$$
H I=\frac{D_{2 \%}-D_{98 \%}}{D_{50 \%}}
$$

where $D_{2 \%}, D_{98 \%}$ and $D_{50 \%}$ are the doses received by $2 \%, 98 \%$ and $50 \%$ of the target volume, respectively. This definition of the HI was used in this study. The HI value can range from 0 to positive infinity. A perfectly homogeneous dose distribution is equivalent to an $\mathrm{HI}$ value of 0 , and it produces a vertical drop on the cumulative DVH curve for the target at the Rx value. Conversely, a very high HI value indicates that the corresponding treatment plan does not meet clinically-acceptable homogeneity standards.

Gradient Index. CI and HI give information regarding the coverage and uniformity of dose inside the target. Gradient index (GI), on the other hand, is used to characterize the steepness of the dose gradient outside the target, and it is defined $\operatorname{as}^{83}$

$$
G I=\frac{V_{50 \%}}{V_{100 \%}}
$$

where $V_{50 \%}$ and $V_{100 \%}$ are the absolute volumes receiving $50 \%$ and $100 \%$ of the $\mathrm{Rx}$. The value of the GI ranges from unity to positive infinity - note that GI can never be smaller than unity since $\mathrm{V}_{100 \%}$ is always encompassed by $\mathrm{V}_{50 \%}$. 
Table 2.4: Most relevant tolerance values for a few OARs surrounding the spine, lung and brain targets. The tolerance values are for conventional fractionation, and an example of the change of the tolerance value with hypofractionation is shown for healthy lungs. $\mathrm{V}_{5 G y}, \mathrm{~V}_{20 G y}$ and $\mathrm{V}_{45 G y}$ are the fractional or absolute volumes of a structure receiving 5, 20 and $45 \mathrm{~Gy}$, respectively. Additional surrounding OARs and additional dose-volume constraints were used during the creation of the treatment plans.

\begin{tabular}{|l|l|l|}
\hline OAR & $\begin{array}{l}\text { Tolerance } \\
\text { value }\end{array}$ & $\begin{array}{l}\text { Increased risk of side effect } \\
\text { beyond tolerance }\end{array}$ \\
\hline Spinal cord & Max dose $\leq 48 \mathrm{~Gy}$ & Myelopathy \\
Kidneys & Mean dose $\leq 18 \mathrm{~Gy}$ & Renal dysfunction \\
Bowel & $\mathrm{V}_{45 \mathrm{~Gy}} \leq 195 \mathrm{~cm}^{3}$ & High-grade bowel toxicity \\
Healthy lungs (conventional) & $\mathrm{V}_{5 \mathrm{~Gy}} \leq 50 \%$ & Radiation pneumonitis \\
& $\mathrm{V}_{20 \mathrm{~Gy}} \leq 30 \%$ & Radiation pneumonitis \\
Healthy lungs (hypofractionated) & $\mathrm{V}_{20 \mathrm{~Gy}} \leq 10 \%$ & Radiation pneumonitis \\
Brainstem & Max dose $\leq 54 \mathrm{~Gy}$ & Necrosis \\
Optic structures & Max dose $\leq 54 \mathrm{~Gy}$ & Blindness \\
\hline
\end{tabular}

OAR doses. The QUANTEC series (Quantitative Analyses of Normal Tissue Effects in the Clinic) $)^{84}$ as well as other sources were used as the reference for OAR radiation tolerances. Listed in Table 2.4 are examples of a few relevant OARs and their recommended tolerances for targets in the spine, ${ }^{85-88} \operatorname{lung}^{89}$ and brain ${ }^{14,90-92}$ cases included in this study. During the treatment planning, all the surrounding OARs were considered (not just those in Table 2.4), and the doses to these OARs were reduced as reasonably as achievable. 


\section{Chapter 3}

\section{Characterization of modern DCAT (mDCAT)}

The two main features of mDCAT in the Monaco TPS version 5.11 are the ability to vary the dose rate during the arc delivery, and the ability of moderate beam modulation through SSO. Moreover, with mDCAT the target-to-MLC margin can be configured to different levels, the number of beam segments can be controlled by choosing the appropriate arc increment, and a structure can be set as an avoidance. In this chapter, a number of representative patients were used to characterize these new features on targets of different sizes and shapes, and different surrounding OARs that require different sets of constraints. The user-manual of Monaco 5.11 offers extremely limited information on mDCAT, and there is no literature on evaluating it, hence the methods used in this study to characterize mDCAT were developed from scratch.

\subsection{Dose rate}

The dose rate for mDCAT planning can be varied or kept constant. The optimal dose rate for each beam segment is generated during the optimization. Given that the gantry speed is fixed for mDCAT arc delivery, varying the dose rate is the computer-equivalent of manually adjusting the beam weight (as done in 3DCRT planning) to account for the different anatomy thicknesses that the beam traverses from different angles around the patient. To understand the behavior of the Monaco TPS when creating mDCAT 
plans with constant dose rate versus variable dose rate, pairs of mDCAT plans (one with constant dose rate and the other with variable dose rate) were created using the same set of objectives and constraints during optimization and the same beam geometry (arc range, collimator angle, arc increment, couch angle). Additionally, the interplay between SSO and dose rate was explored by creating pairs of mDCAT plans without and with SSO.

Shown in Figure 3.1 are the dose distributions and DVHs of the PTV and the OARs for different combinations of dose rate and SSO for an example spine case. These plans were optimized using the same cost functions, and the optimization was set to "constrained" mode, which effectively served as an implicit normalization such that the OARs were spared the best possible way given the set of constraints (refer to Section 2.2.1).

For constant dose rate without and with SSO (Figures 3.1a and 3.1b), the plans were substantially inferior in terms of target coverage where there was no $100 \%$ isodose color-wash present in the dose distributions. With the use of a variable dose rate without and with SSO, about $90 \%$ and $95 \%$ of the PTV volume was covered by the $\mathrm{Rx}$, respectively, which was a big improvement in the target coverage compared to that for constant dose rate. In terms of OAR sparing with constant dose rate, in addition to the substantially worse PTV dose coverage, OARs were receiving more of the lower doses. Figure 3.2 shows the same plans of Figure 3.1 but normalized such that $95 \%$ of the PTV volume is forced to receive the $\mathrm{Rx}$, which is the clinically-acceptable standard for PTV coverage. For acceptable clinical coverage, the OAR dose is substantially more with constant dose rate, regardless of SSO. The addition of SSO (regardless of dose 

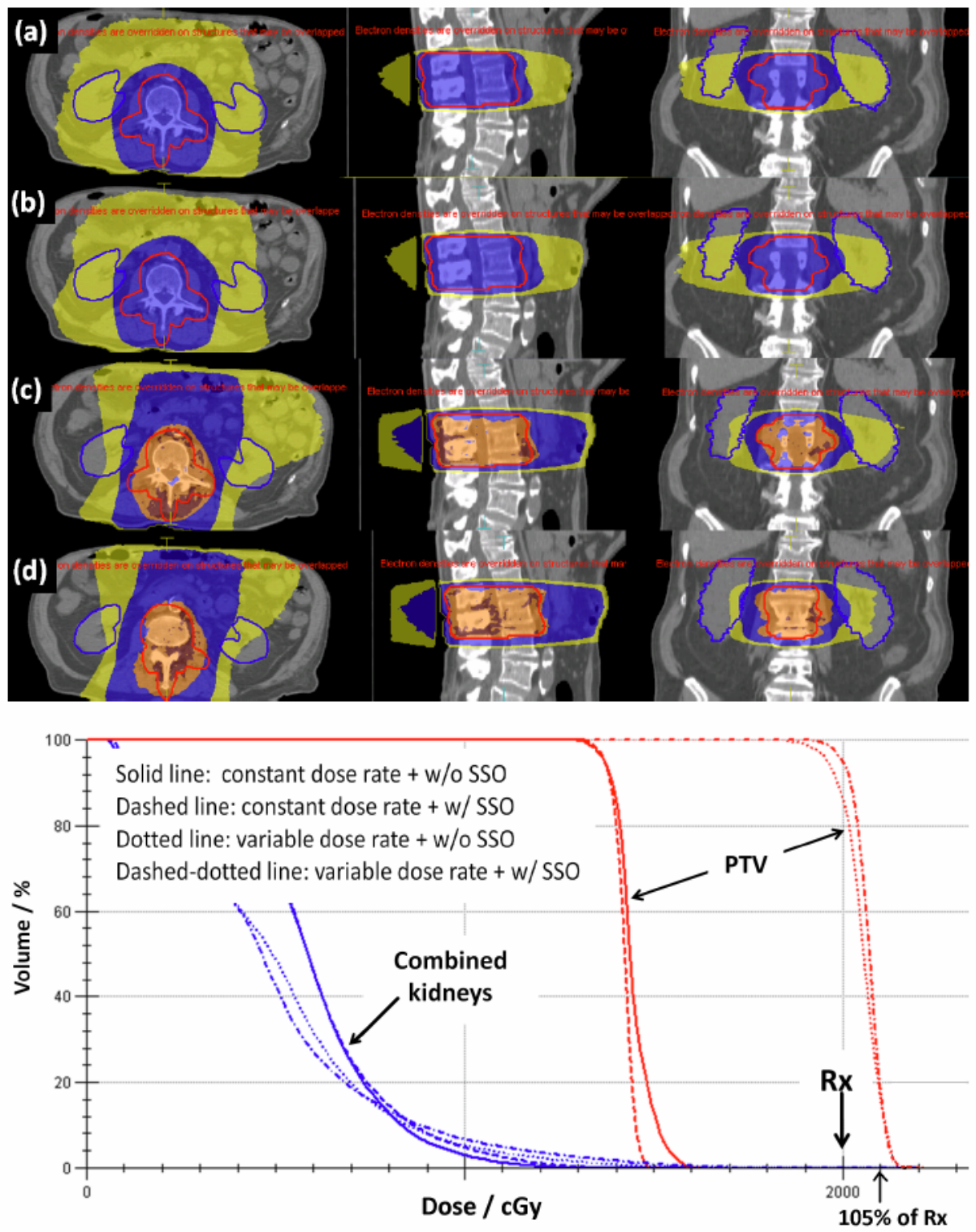

Figure 3.1: For an example palliative spine patient, the figure shows dose distributions in the axial (left), sagittal (middle) and coronal (right) CT views for mDCAT plans with different combinations of dose rate and SSO: (a) constant dose rate without SSO, (b) constant dose rate with $\mathrm{SSO}$, (c) variable dose rate without SSO, and, (d) variable dose rate with SSO. All plans were optimized using the same cost functions and implicitly normalized during the optimization to emphasize OAR sparing. The color-washes represent the following percent of the Rx: $25 \%$ (yellow), $50 \%$ (blue), $100 \%$ (orange) and 105\% (brown). The bottom panel shows the DVHs for the PTV (red) and the combined kidneys (blue) for the four combinations of dose rate and SSO. The Rx is 2000 cGy in 5 fractions. 

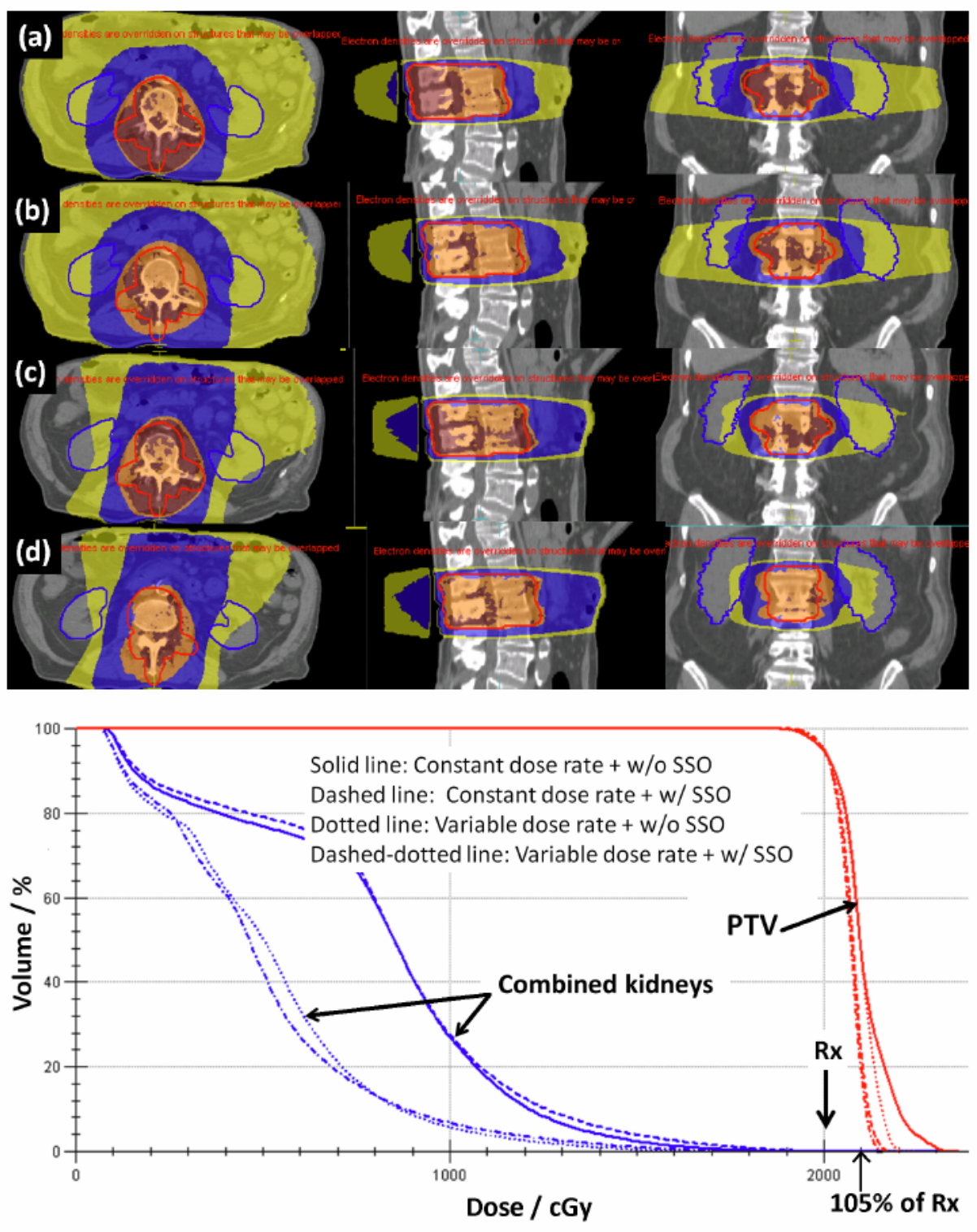

Figure 3.2: Same as in Figure 3.1, but with the normalization $\mathrm{D}_{95 \%}=\mathrm{Rx}$ for four cases individually. 
rate) reduces the hotspots as seen on the tail of the DVH at the higher doses (compare the pairs without versus with SSO for a given dose rate choice), which is reflected on the size of the $105 \%$ isodose area in the dose distributions.

The combined improvement from variable dose rate and SSO supports the motivation of this study to investigate the potential of mDCAT with variable dose rate and SSO, which are features that were not available in cDCAT.

\subsection{Target-to-MLC margin}

The target-to-MLC margin for an mDCAT plan can be set to five different levels, namely, very tight $(0$ to $1 \mathrm{~mm})$, tight $(2 \mathrm{~mm})$, narrow $(3$ to $4 \mathrm{~mm})$, normal $(8 \mathrm{~mm})$ and wide $(10 \mathrm{~mm})$. The impact of the size of the PTV-to-MLC margin on plan quality was evaluated by repeating the generation of different treatment plans with SSO using different margins. The plans were normalized so that $95 \%$ of the PTV volume receives the $\operatorname{Rx}\left(D_{95 \%}=R x\right)$.

Shown in Figure 3.3 are dose distributions and DVHs for the PTV and an OAR for different combinations of target-to-MLC margin for an example lung SBRT patient. For the very tight target-to-MLC margin, the Rx isodose line conformed very well to the PTV, which resulted in hotspots in the middle of the PTV. For the wide target-to-MLC margin, the hotspots were reduced. However, the Rx isodose line was spilling outside the PTV boundaries into the surrounding OARs. The magnitude of the hotspots was reflected in the DVH curve for the PTV, in which the tail of the curve was much longer for very tight target-to-MLC margin compared to that with wide margin. The behavior of the DVH tail was found to be inversely dependent on the PTV size for a given target-to-MLC margin. For example, the tail of the DVH curve for the PTV is less than $110 \%$ Rx for large palliative spine targets, but can reach $130 \% \mathrm{Rx}$ for small lung 

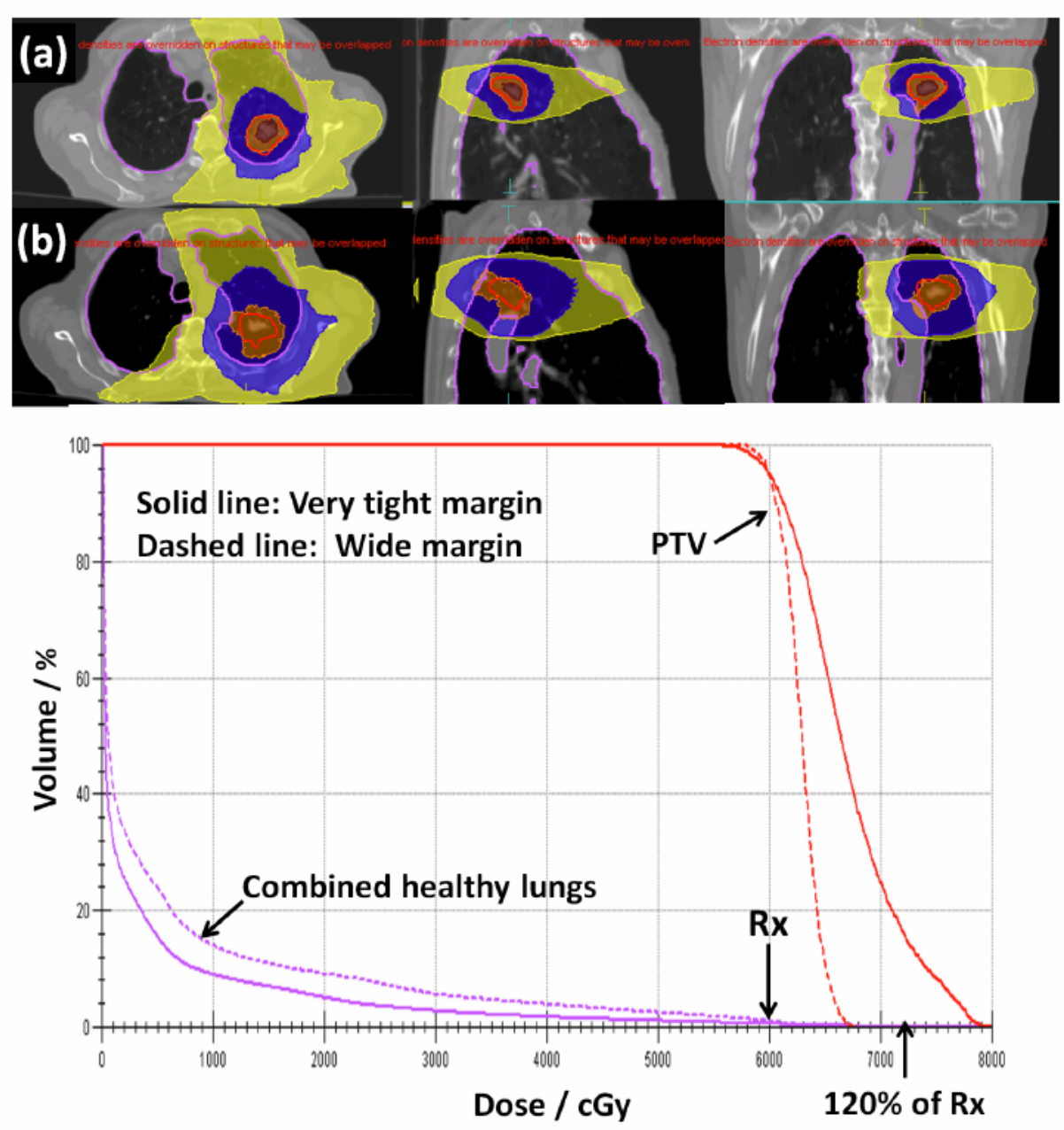

Figure 3.3: For an example lung SBRT patient, the figure shows dose distributions in the axial (left), sagittal (middle), and coronal (right) CT views for mDCAT with SSO plans for different combinations of target-to-MLC margin: (a) very tight margin, and, (b) wide margin. The color-washes represent the following percent of the Rx: $25 \%$ (yellow), $50 \%$ (blue), $100 \%$ (orange) and $120 \%$ (brown). The bottom panel shows the DVHs for the PTV (red) and the combined healthy lungs (purple). The Rx is 6000 cGy in 8 fractions. The relative behaviour for mDCAT without SSO for different target-to-MLC margins is very comparable to the relative behaviour shown in this figure.

SBRT and brain targets. Depending on the site and type of treatment (e.g., SBRT or not), hotspots of up to $25 \%$ may or may not be acceptable. It was shown here that there was a trade-off between dose homogeneity and dose conformality. The two tightest margins were used throughout the study for conformality. 


\subsection{Arc increment}

For both mDCAT and VMAT planning, the user defines an arc increment. For mDCAT with constant dose rate (regardless of the SSO choice), the optimizer creates two segments per arc increments, where each segment spans half the arc increment. So for instance, for a $360^{\circ}$ delivery and an arc increment of $20^{\circ}$, a total of $360 / 20 * 2=$ 36 segments are generated. On the other hand, for mDCAT with variable dose rate (regardless of the choice of SSO) and VMAT, the arc increment defines the limits of the sweeping motion of the MLCs during the delivery, and any number of segments can be generated within each arc increment.

For both mDCAT and VMAT, choosing an arc increment that is too large can lead to lower quality plan, while choosing an arc increment that is too small can lead to longer optimization time without an improvement to the plan quality. To evaluate the impact of arc increment on plan quality, treatment plans for mDCAT and VMAT were generated using three arc increments of $5^{\circ}, 10^{\circ}$ and $20^{\circ}$. For the mDCAT plans, both variable dose rate and SSO were used to achieve the best possible mDCAT plan quality (as discussed in Section 3.1).

Reducing the arc increments from $20^{\circ}$ to $10^{\circ}$ and to $5^{\circ}$ increased the treatment plan optimization times by approximately factors of 1.5 and 2.5 , respectively. The three arc increments offered very similar target coverage and OAR sparing. There was a marginal improvement in the OAR sparing for the smaller arc increments. Arc increments larger than 20 degrees were found to reduce the plan quality particularly for cases with small targets. Therefore, the mDCAT and VMAT plans for the rest of this study were generated using $20^{\circ}$ arc increment for more efficient plan optimization without compromising plan quality. 


\subsection{Avoidance structure}

A structure can be assigned as an avoidance if the desire is to totally spare the structure. This completely blocks the organ with the MLC leaves, which avoid the entrance or exit of the beam to the structure. An additional margin can be added to increase the extent of blockage by the MLC leaves. This margin has the same five levels used in configuring the target-to-MLC margin. It was found that assigning a structure that was in the vicinity of the target volume as an "avoidance structure" severely degraded the plan quality, therefore this feature was not used for the mDCAT plans in this study.

\subsection{Segment Shape Optimization (SSO)}

SSO is an optional refinement available to the user for both mDCAT and VMAT. With $\mathrm{SSO}$, the generated beam segments from the initial optimization step for both mDCAT and VMAT are refined (see Section 2.2.2) through five cycles of SSO that include fluence smoothing, aperture sequencing or clustering, and optimization of beam weights and shapes in order to improve plan quality, delivery smoothness, and delivery efficiency. The addition of SSO is the key to the added modulation in mDCAT (versus cDCAT).

For mDCAT with constant dose rate and SSO, the number of beam segments was found to be constant between each cycle of the SSO. For mDCAT with variable dose rate and SSO, the number of segments could vary between each cycle of SSO. For instance, for a representative lung SBRT case with a small PTV, the initial 88 beam segments (which was the number of segments without SSO) changed to 80, 80, 79, 78 and 78 segments going from the first to the fifth cycle of SSO. Other plan aspects can also be changed by the optimizer during SSO, including the number of MUs, the start and end angles of each segment, the jaw positions for different segments, and the dose rate. 
The MLC is the key LINAC component that allows for modulation during the five cycles of SSO, and the solid jaws contribute to a lesser extent. To characterize the modulation in mDCAT with SSO for different PTV sizes and surrounding OARs, the displacements of the MLC leaves were calculated after each cycle of SSO (relative to their positions before the start of SSO) for lung SBRT cases (small targets) and for palliative spine cases (large targets) once using very tight target-to-MLC margins and once using wide margins.

Shown in Figure 3.4 are the histograms for the MLC displacements after the first and the fifth cycle of SSO for example lung SBRT and palliative spine cases. For the example lung SBRT case with a very tight margin, there was a gradual increase in the number of active MLC leaves that are moving away from the target edge (or "opening"). The explanation for this behaviour is that lungs have lower density, which reduces the dose buildup to the PTV in the lung region. The restrictive distance between the PTV and the MLC for very tight margin was not enough to buildup the dose, and to compensate for this lack of buildup, the MLC leaves were "opened" by the optimizer during SSO. For the same patient but with a wide target-to-MLC margin during planning, the fraction of active MLC leaves "opening" decreased, with some MLC leaves "closing".

For the example palliative spine case, because of the size of the PTV and the details of the surrounding OARs, the MLC leaves could move towards or move away from the PTV edge throughout the five cycles of SSO, regardless of the target-to-MLC margin size (very tight or wide).

For both small (such as in lung SBRT) and large (such as in spine) PTVs, the maximum MLC leaf displacement observed was $24 \mathrm{~mm}$. This moderate extent of MLC displacement highlights the difference in the degree of modulation between mDCAT 


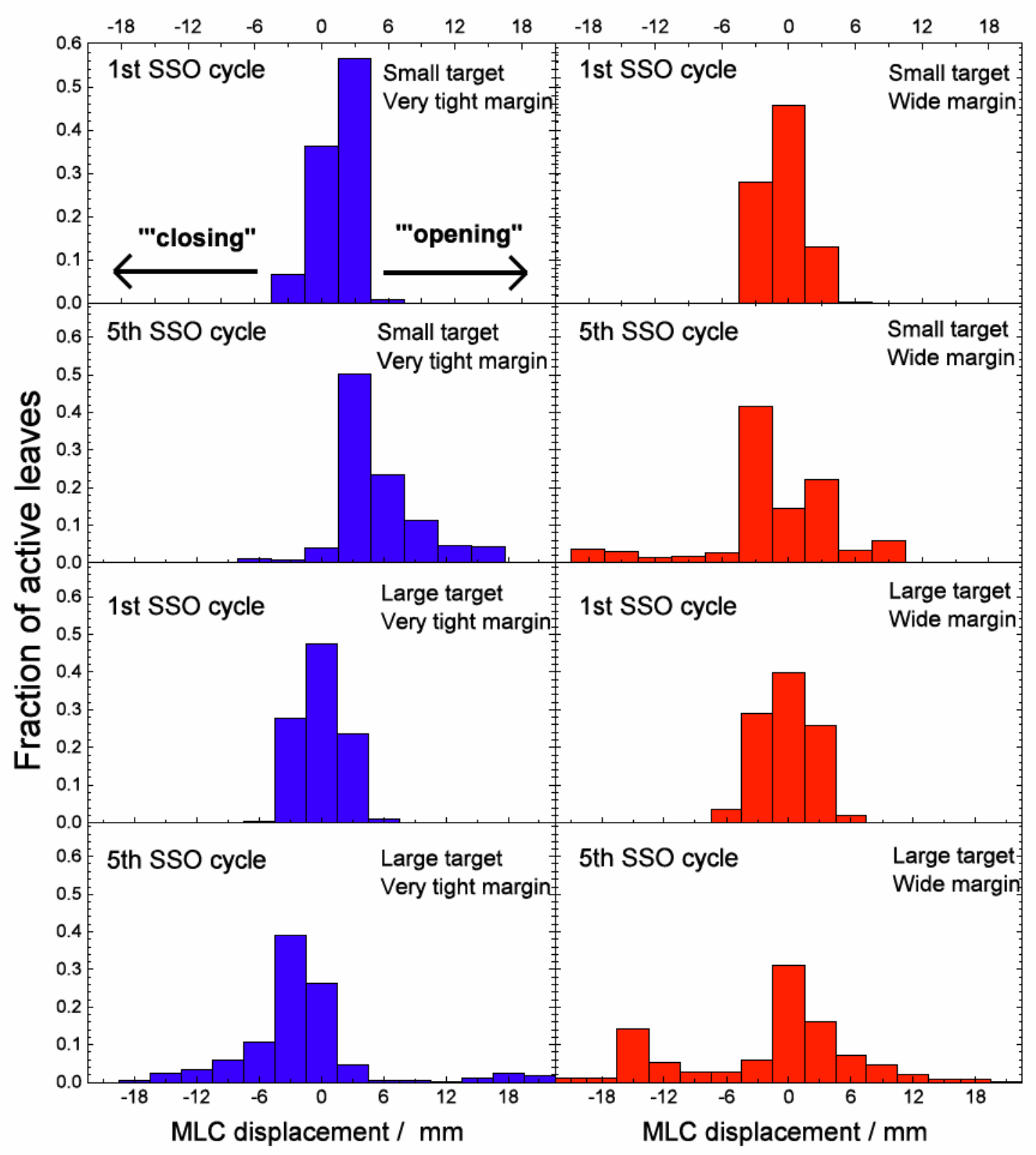

Figure 3.4: Typical distribution of MLC leaf displacement for a small PTV (top 4 panels) and for a large PTV (bottom 4 panels) after the first and the fifth cycles of SSO for mDCAT plans using very tight (left) and wide (right) target-to-MLC margins. The displacement is relative to the MLC leaf positions before the start of SSO. Only the active MLC leaves within the open field are in the histogrammed data. Positive displacement (or "opening") means that the MLC leaves moved away from the PTV edge, while negative displacement (or "closing") means that the MLC leaves moved towards the PTV edge. 

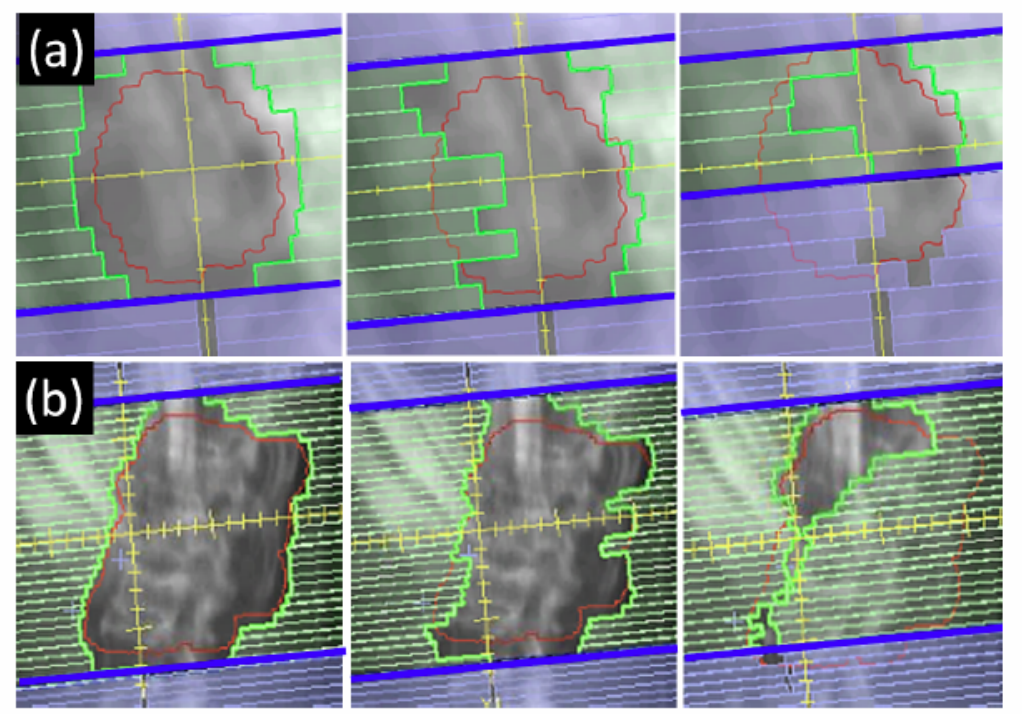

Figure 3.5: BEV of a beam segment for mDCAT without SSO (left), mDCAT with SSO (middle), and, VMAT (right). The top row (a) is for a typical lung SBRT patient with a small target, and the bottom row (b) is for a typical palliative spine patient with a large target. For the dimensional scale, the thickness of each green MLC strip is $5 \mathrm{~mm}$ in both rows.

and VMAT, particularly for large targets. Although the same optimizer was used to generate the mDCAT and VMAT segments, the initial segments for mDCAT conform to the target and so the refinement is just around the target periphery. On the other hand, for VMAT the initial segments can be of any shape, and they get modified during SSO starting from those initial shapes.

Shown in Figure 3.5 are the Beam's Eye Views (BEVs) of starting position of a beam segment for mDCAT and VMAT plans. For mDCAT without SSO (left), and regardless of the target size, the MLC leaves were shaped around the PTV with the configured target-to-MLC margin. When the plan underwent SSO (middle), the MLC leaves moved away from or towards the target edge beyond the configured targetto-MLC margin, covering some parts around the periphery of the PTV. Comparing mDCAT with SSO (middle) versus VMAT (right), the difference in modulation between the two techniques is more obvious for larger targets (bottom row) than it is for smaller targets (top row). 


\subsection{Summary}

The use of variable dose rate in mDCAT gives superior plan quality compared to the plan quality achievable with constant dose rate. Using the very tight target-to-MLC margin resulted in the most conformal dose distributions, which spare more of the surrounding healthy organs, but at the expense of reduced target dose homogeneity. Completely blocking a structure in the vicinity of the target volume severely degrades the target dose coverage. Using an arc increment up to 20 degrees improves the plan optimization efficiency without compromising the plan quality. The addition of SSO in mDCAT planning improves the plan quality by allowing for moderate beam modulation using the MLC leaves, mostly around the target periphery. During SSO in mDCAT, the MLC leaves can move up to only $2.4 \mathrm{~cm}$ beyond the set target-to-MLC margin to improve plan quality. This moderate degree of modulation for mDCAT is in contrast with VMAT, particularly for large targets.

With this understanding of the different features of mDCAT, in the next four chapters, the performance of mDCAT is assessed against 3DCRT and VMAT in terms of plan optimization and treatment delivery efficiency, plan quality, and plan robustness against machine and patient uncertainties. 


\section{Chapter 4}

\section{Efficiency of plan optimization and treat- ment delivery}

With the moderate modulation in mDCAT (see Chapter 3), the optimization of an mDCAT plan in the Monaco TPS is expected to be more efficient than it is for VMAT, which has a larger solution space during optimization due to the higher degrees of freedom the LINAC offers during delivery. The treatment delivery for mDCAT is also expected to be faster because of the moderate beam modulation. On the other hand, mDCAT being an arc delivery is expected to deliver more conformal dose to the target when compared to the dose delivered with 3DCRT. This potential combination of efficiency and conformality can make mDCAT a good option for rapid-access high-quality palliative RT.

\subsection{Optimization time}

In this chapter, unless explicitly stated otherwise, the term "optimization" includes all the steps involved: ideal photon fluence generation and/or segmentation, SSO, and final dose calculations.

To evaluate optimization times, treatment plans were created for the patient database described in Section 2.5, which includes twenty five palliative spine, twelve lung SBRT, and twelve brain patients. The methods of planning for each disease site were de- 
scribed earlier in Section 2.6. In the mDCAT plans, variable dose rate without and with SSO was explored, and tight or very tight target-to-MLC margins were used for better conformality. For the twenty five palliative spine patients, the effect of grid size on the optimization time was assessed by performing the calculations at both $3 \mathrm{~mm}^{3}$ and $5 \mathrm{~mm}^{3}$ voxel sizes for the mDCAT and VMAT plans, with all other parameters unchanged. The larger grid size was explored because it leads to faster plan optimization and faster dose calculation, which is very relevant for rapid-access palliation of patients with spine metastases. The trade-off when using a larger grid size is a coarser dose grid and higher volume-averaging effects, which can be clinically acceptable for rapid-palliative situations

The start and end times of the optimization were collected from the TPS log files. The fractional time spent on optimization excluding the final dose calculation was also recorded and compared for mDCAT versus VMAT. The computer times were for optimization using the final set of objectives and constraints. The human time invested in reaching this suitable set of objectives and constraints was not included, which is not an issue for situations that require prompt treatment since pre-determined disease-sitespecific set of objectives and constraints are typically in a template for quick use with only minor adjustments. Since mDCAT plans are simpler than VMAT plans, the set of objectives and constraints are also simpler, and thus customizing them for each patient requires only minimal modifications to the template set, which is an additional gain in efficiency for mDCAT versus VMAT beyond what is reported in this study.

The results of the optimization efficiency are shown in Figure 4.1 and Tables 4.1 to 4.4. Shown in Figure 4.1 are the boxplots for the absolute and relative optimization times for mDCAT without SSO, mDCAT with SSO, and VMAT. Shown in these boxplots are the median (middle line), the 25th and the 75th quartiles (box edges), the 1.5 Inter- 
Quartile Range - IQR (whisker, which represents the variability outside the upper and lower quartiles), and the outliers (points outside the whiskers). This boxplot configuration is the one used for all the boxplots presented in this study. For the palliative spine and brain cases (Tables 4.1 and 4.4), the addition of SSO on mDCAT plans increased the optimization time on average by a factor of two, and it took about three times longer for VMAT plans to optimize compared to mDCAT without SSO. For the lung SBRT cases (Table 4.3), the optimization times for mDCAT with SSO and VMAT relative to the optimization time for mDCAT without SSO were higher than those for the spine and brain cases. The higher normalized optimization times for the lung cases can be explained as follows. With the initial beam segments just conforming to the target with some margin (the stage before SSO for mDCAT), these initial beam segments needed more refinements in order to meet sufficient target coverage given the lack of dose buildup for the lung cases. This was also reflected on the higher fractional time spent on optimization alone for the lung cases, compared to those for the spine and brain cases.

Changing the voxel size from $3 \mathrm{~mm}^{3}$ to $5 \mathrm{~mm}^{3}$ (Table 4.2 ) reduced the average time for mDCAT w/o SSO to under 3 minutes for the twenty five palliative spine patients. Although the absolute times changed when the grid size was varied, the normalized optimization times for mDCAT with SSO and VMAT relative to mDCAT without SSO remained the same.

Figure 4.2 shows scatter plots for the optimization time for mDCAT with SSO and VMAT normalized to the time for mDCAT without SSO as a function the PTV size for the twenty five palliative spine, twelve lung SBRT and twelve brain patients. The values of the corresponding correlation coefficients are shown in Table 4.5. The correlation is very weak for spine and lung SBRT, and moderate for brain. This indicates that, in 

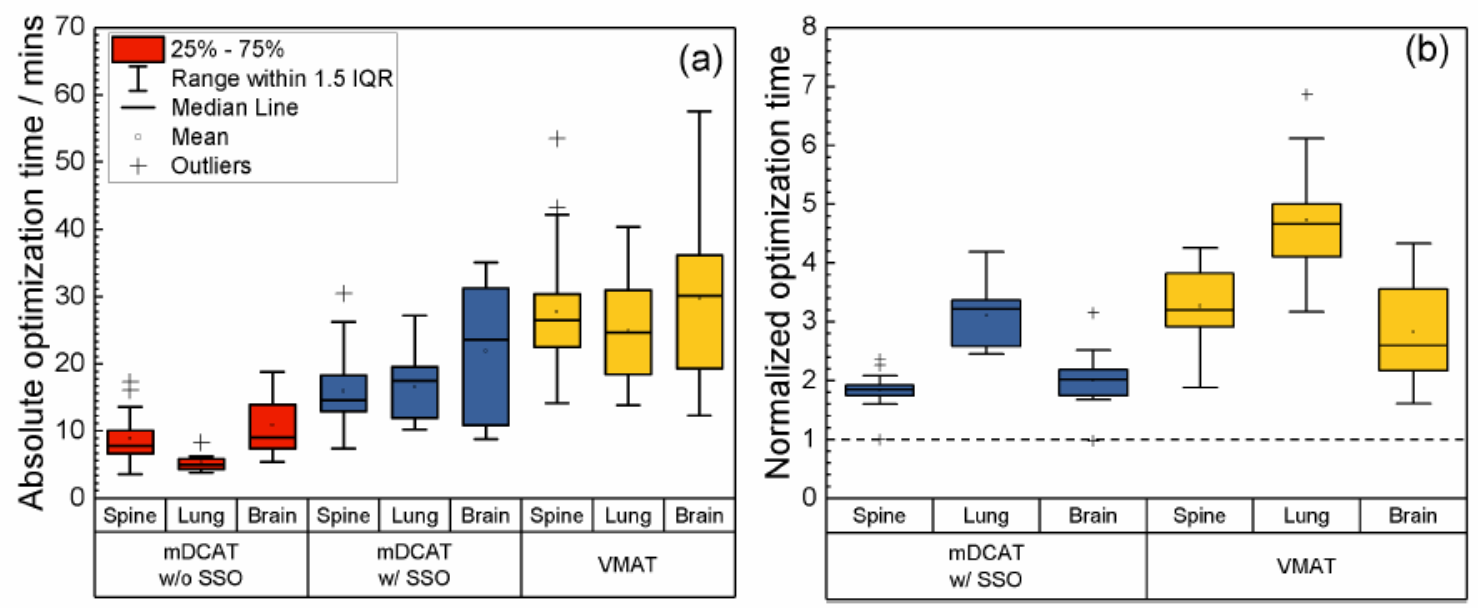

Figure 4.1: (a) Absolute optimization times for mDCAT without SSO, mDCAT with SSO, and VMAT treatment plans for the twenty palliative spine, twelve lung SBRT and twelve brain patients included in this study. (b) Optimization times for mDCAT with SSO and VMAT normalized to the optimization time for mDCAT without SSO (dashed line). The calculation voxel size is $3 \mathrm{~mm}^{3}$.

Table 4.1: Average absolute and normalized optimization times and fractional times spent on optimization excluding final dose calculations for mDCAT and VMAT for the twenty five palliative spine patients included in this study. The calculation voxel size is $3 \mathrm{~mm}^{3}$.

\begin{tabular}{|l|c|c|c|}
\hline Technique & $\begin{array}{c}\text { Absolute time } \\
\text { (minutes) }\end{array}$ & $\begin{array}{c}\text { Normalized time to } \\
\text { mDCAT without SSO }\end{array}$ & $\begin{array}{c}\text { Fractional time spent } \\
\text { on optimization }\end{array}$ \\
\hline mDCAT w/o SSO & $8.1 \pm 3.3$ & $1.0 \pm 0.0$ & 0.61 \\
mDCAT w/ SSO & $14.0 \pm 5.0$ & $1.8 \pm 0.3$ & 0.79 \\
VMAT & $25.8 \pm 8.7$ & $3.3 \pm 0.6$ & 0.86 \\
\hline
\end{tabular}

Table 4.2: Average absolute and normalized optimization times for mDCAT and VMAT for the twenty five palliative spine patients included in this study. The calculation voxel size is $5 \mathrm{~mm}^{3}$.

\begin{tabular}{|l|c|c|}
\hline Technique & $\begin{array}{c}\text { Absolute time } \\
\text { (minutes) }\end{array}$ & $\begin{array}{c}\text { Normalized time to } \\
\text { mDCAT without SSO }\end{array}$ \\
\hline mDCAT w/o SSO & $2.7 \pm 1.0$ & $1.0 \pm 0.0$ \\
mDCAT w/ SSO & $5.4 \pm 1.9$ & $2.0 \pm 0.2$ \\
VMAT & $9.3 \pm 3.4$ & $3.5 \pm 0.5$ \\
\hline
\end{tabular}


Table 4.3: Same as in Table 4.1, but for the twelve lung SBRT patients included in this study.

\begin{tabular}{|l|c|c|c|}
\hline Technique & $\begin{array}{c}\text { Absolute time } \\
\text { (minutes) }\end{array}$ & $\begin{array}{c}\text { Normalized time to } \\
\text { mDCAT without SSO }\end{array}$ & $\begin{array}{c}\text { Fractional time spent } \\
\text { on optimization }\end{array}$ \\
\hline mDCAT w/o SSO & $5.3 \pm 1.3$ & $1.0 \pm 0.0$ & 0.65 \\
mDCAT w/ SSO & $16.6 \pm 5.3$ & $3.1 \pm 0.5$ & 0.89 \\
VMAT & $25.0 \pm 8.1$ & $4.7 \pm 1.1$ & 0.87 \\
\hline
\end{tabular}

Table 4.4: Same as in Table 4.1, but for the twelve brain patients included in this study.

\begin{tabular}{|l|c|c|c|}
\hline Technique & $\begin{array}{c}\text { Absolute time } \\
\text { (minutes) }\end{array}$ & $\begin{array}{c}\text { Normalized time to } \\
\text { mDCAT without SSO }\end{array}$ & $\begin{array}{c}\text { Fractional time spent } \\
\text { on optimization }\end{array}$ \\
\hline mDCAT w/o SSO & $11.0 \pm 4.7$ & $1.0 \pm 0.0$ & 0.58 \\
mDCAT w/ SSO & $21.9 \pm 9.9$ & $2.0 \pm 0.5$ & 0.82 \\
VMAT & $29.8 \pm 13.5$ & $2.8 \pm 0.9$ & 0.81 \\
\hline
\end{tabular}
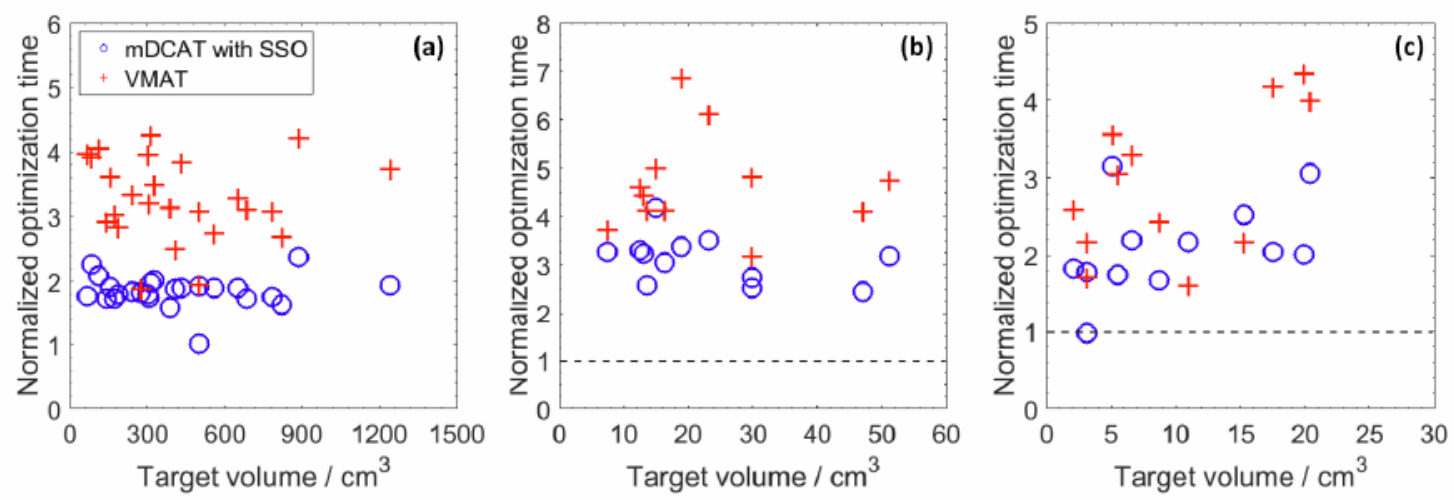

Figure 4.2: Scatter plots to evaluate the correlation between target size and optimization time for mDCAT with SSO and VMAT normalized to the time for mDCAT without SSO (dashed line) for the (a) twenty five palliative spine, (b) twelve lung SBRT, and (c) twelve brain patients.

general, all target sizes can equally benefit from the reduced optimization time with mDCAT. 
Table 4.5: Correlation coefficients between target size and optimization time for mDCAT with SSO and VMAT normalized to the time for mDCAT without SSO for the twenty five palliative spine, twelve lung SBRT, and twelve brain patients.

\begin{tabular}{|l|c|c|c|}
\hline \multirow{2}{*}{ Technique } & \multicolumn{3}{|c|}{ Correlation coefficients } \\
\cline { 2 - 4 } & Spine & Lung SBRT & Brain \\
\hline mDCAT w/ SSO & -0.03 & -0.40 & 0.44 \\
VMAT & -0.39 & -0.04 & 0.58 \\
\hline
\end{tabular}

\subsection{Treatment delivery time}

To evaluate delivery times for different disease sites, target sizes, and fractionation, the Elekta Synergy/Infinity LINAC was used to deliver mDCAT with SSO and VMAT plans for six palliative spine, six lung SBRT and six brain patients.

The results comparing the treatment delivery times for mDCAT with SSO and VMAT are shown in Tables 4.6. One of the six lung SBRT patients (not included in Table 4.6) had a daily dose of 1200 cGy, and the delivery times for mDCAT with SSO and VMAT were 5.7 and 6.6 minutes, respectively. In general, treatment delivery times are short, and so the absolute gains of treating with mDCAT versus VMAT are small, except for rapid-access palliative situations where the process of scan-plan-treat is to be done in a typical treatment time slot of around 30 minutes. The faster delivery also reduces the total intra-faction patient motion and its impact on dose delivery accuracy.

Table 4.6: Average treatment time for mDCAT with SSO and VMAT and their standard deviations for the six palliative spine, six lung SBRT, and six brain plans delivered on an Elekta Synergy LINAC at TOHCC.

\begin{tabular}{|c|c|c|c|}
\hline \multirow[b]{2}{*}{ Technique } & \multicolumn{3}{|c|}{ Treatment time (mins) } \\
\hline & $\begin{array}{c}\text { Spine } \\
400 \mathrm{cGy} / \mathrm{fx}\end{array}$ & $\begin{array}{l}\text { Lung SBRT } \\
750 \mathrm{cGy} / \mathrm{fx}\end{array}$ & $\begin{array}{c}\text { Brain } \\
800 \mathrm{cGy} / \mathrm{fx}\end{array}$ \\
\hline mDCAT $w /$ SSO & $1.5 \pm 0.1$ & $2.5 \pm 0.5$ & $3.0 \pm 0.4$ \\
\hline VMAT & $2.2 \pm 0.5$ & $3.6 \pm 0.5$ & $3.7 \pm 0.7$ \\
\hline
\end{tabular}


Table 4.7: Modulation factor and its standard deviation for mDCAT with SSO, and for VMAT. The modulation factor is defined as the ratio of the number of MUs for mDCAT with SSO or for VMAT, relative to the number of MUs for mDCAT without SSO (which has no modulation). Data are shown for the twenty five palliative spine, twelve lung SBRT, and twelve brain cases included in this study.

\begin{tabular}{|l|c|c|c|}
\hline \multirow{2}{*}{ Technique } & \multicolumn{3}{|c|}{ Modulation factor } \\
\cline { 2 - 4 } & Spine & Lung SBRT & Brain \\
\hline mDCAT w/ SSO & $1.01 \pm 0.03$ & $1.00 \pm 0.03$ & $1.00 \pm 0.03$ \\
VMAT & $1.58 \pm 0.40$ & $1.26 \pm 0.25$ & $1.35 \pm 0.26$ \\
\hline
\end{tabular}

\subsection{Monitor units and modulation factor}

A lower number of MUs is desirable for faster treatment delivery. Lower MUs also leads to less LINAC head leakage and patient scatter, which can reduce the probability of radiation-induced secondary cancers. ${ }^{93}$ More modulation (such as in VMAT) leads to a larger the number of MUs to deliver the same dose. In this study, the "modulation factor" for a particular treatment plan is defined as the ratio of the MUs for that plan relative to the MUs for the corresponding mDCAT plan without SSO. The mDCAT without SSO was taken as the base plan for comparison because the field shapes conform to the target boundaries throughout the arc delivery, without any modulation. The modulation factor was calculated for the twenty five palliative spine, twelve lung SBRT and twelve brain patients included in this study, and the results are shown in Table 4.6. The results show that for mDCAT, the MUs without and with SSO are very comparable. For VMAT, $35 \%$ to $55 \%$ more MUs were needed versus the MUs required for mDCAT..

\subsection{Summary}

mDCAT (without and with SSO) is two to three times more efficient than VMAT in terms of optimization time. The average optimization time for mDCAT can be reduced 
to under three minutes using a clinically acceptable set of calculation parameters. The average treatment delivery times for mDCAT and VMAT are short, and so the absolute gains of treating with mDCAT versus VMAT are small. Efficient optimization and efficient treatment delivery become clinically relevant for rapid-access palliative situations where the process of scan-plan-treat is to be done in a typical treatment time slot of around 30 minutes. The number of MUs required for mDCAT is substantially less than that required for VMAT, which translates into less head leakage and patient scatter to body areas outside the treatment fields. 


\section{Chapter 5}

\section{Plan quality}

In this chapter, the plan quality for mDCAT (without and with SSO) versus 3DCRT and VMAT were evaluated for the palliative spine, lung SBRT and brain cases (see Section 2.5 for patient selection).

\subsection{Plan quality for spine}

The results of comparing the plan quality for mDCAT without and with SSO versus 3DCRT and VMAT for the twenty five palliative spine patients are shown in Table 5.1 and in Figures 5.1 and 5.2. Table 5.1 shows the average plan quality metrics and their standard deviations, and Figures 5.1 and 5.2 show boxplots of the plan quality metrics, and dose to the relevant OARs, respectively.

From Table 5.1 and Figure 5.1a, the $\mathrm{D}_{2 \%}$ of the PTV produced with mDCAT plans was midway between that for 3DCRT and VMAT plans, and with lowest (and best) $\mathrm{D}_{2 \%}$ for VMAT plans. There was a slight improvement in the $\mathrm{D}_{2 \%}$ for mDCAT with SSO versus that without SSO. One of the reasons for the magnitude of the $\mathrm{D}_{2 \%}$ in mDCAT plans is as follows. In mDCAT the dose rate can be varied between segments using variable dose rate, but it remains constant for any given segment. This means that for mDCAT cases with large variation in patient thickness across a segment (as in the sloping of the anterior chest area when treating the thoracic spine), a larger number 
of MUs is required to deliver the Rx to the PTV at the thicker regions within the treatment area, which consequently delivers (unnecessary) higher dose to the thinner regions. A strategy to address this limitation of mDCAT for large targets spanning over variable patient thickness was to create two treatment plans: the first plan delivering radiation to the whole PTV, while the second plan boosting parts of the PTV at the thicker regions of the treatment area, and the total dose delivered from the two plans was equal to the Rx. However, due to the relatively small weight of the boost plan (about $5 \%$ to $8 \%$ of the $\mathrm{Rx}$ ), the minimum number of MUs deliverable by the physical machine per segment was violated and rendered such strategy infeasible. Shortening the boost arc was not sufficient for overcoming the minimum MU limitation for some of the segments. An example is the patient with the outlier $(+)$ in the boxplot for $\mathrm{D}_{2 \%}$ in Figure 5.1 for mDCAT without and with SSO, which has the PTV between the junction of the neck and shoulder of the patient.

The PTV mean dose, CI, HI and GI (defined in Section 2.7) for the mDCAT plans were in between those achieved with 3DCRT and VMAT plans, with a slight improvement for mDCAT with SSO versus without SSO. The use of arc treatment deliveries (VMAT or mDCAT) substantially improved the PTV dose conformity and homogeneity versus what was achievable with 3DCRT, with mDCAT slightly worse than VMAT. The gradient outside of the PTV (quantified by the GI) was steepest for 3DCRT, which was because of the close volumes of the $50 \%$ and $100 \%$ isodose lines produced with the parallel-opposed beam arrangement for 3DCRT plans. Comparing the two arc deliveries, VMAT and mDCAT plans, on average, produced comparable dose gradient outside the PTV.

For OAR doses (Figure 5.2), mDCAT performed midway in terms of sparing the spinal cord and bowel for PTVs in the cervical and sacral spine, respectively. The more 
homogeneous dose within the PTV for mDCAT and VMAT allowed for more sparing of OARs that fall within the PTV, such as the spinal cord shown in Figure 5.2a, versus the sparing offered by 3DCRT, with VMAT distinctly the best. The dose near the entry points of the beam was substantially reduced with VMAT and mDCAT compared to 3DCRT, such as for the bowel dose shown in Figure 5.2d, with VMAT being slightly better. The dose to the lungs for PTVs in the thoracic spine were highest with mDCAT (regardless of SSO), as shown in Figure 5.2b. Similar results were obtained for dose to the kidneys for PTVs in the lumbar spine, as shown in Figure 5.2c. The large "whiskers" for the lungs and kidneys with mDCAT are due to varying thicknesses across different PTV sizes and across different patients. The results in panels b and $\mathrm{c}$ can be explained as follows. The entry of radiation from all directions with the full arc mDCAT caused the spillage of low doses to lateral organs such as the lungs and the kidneys, which was not present in the parallel-opposed 3DCRT plans because such plans have no beams directly entering through the lungs or kidneys. The spillage to the lateral organs was not present for VMAT because of the high degree of modulation with VMAT deliveries that can carve the delivered dose away from the OARs. A compromise between the 3DCRT and full arc mDCAT could be the use of a partial arc (or a bow-tie) geometry for mDCAT, or to tighten the constraints in the full arc mDCAT plan. Either of these approaches reduces the OAR doses, but at the expense of the target dose.

Overall, for large targets such as those for palliative spine cases, the plan quality achievable with mDCAT is better than that for 3DCRT, but slightly worse than that with VMAT, with marginal improvement in mDCAT with the addition of SSO. The dose distributions and DVHs of the PTV and OARs for an example palliative spine patient are shown in Figure 5.3. 

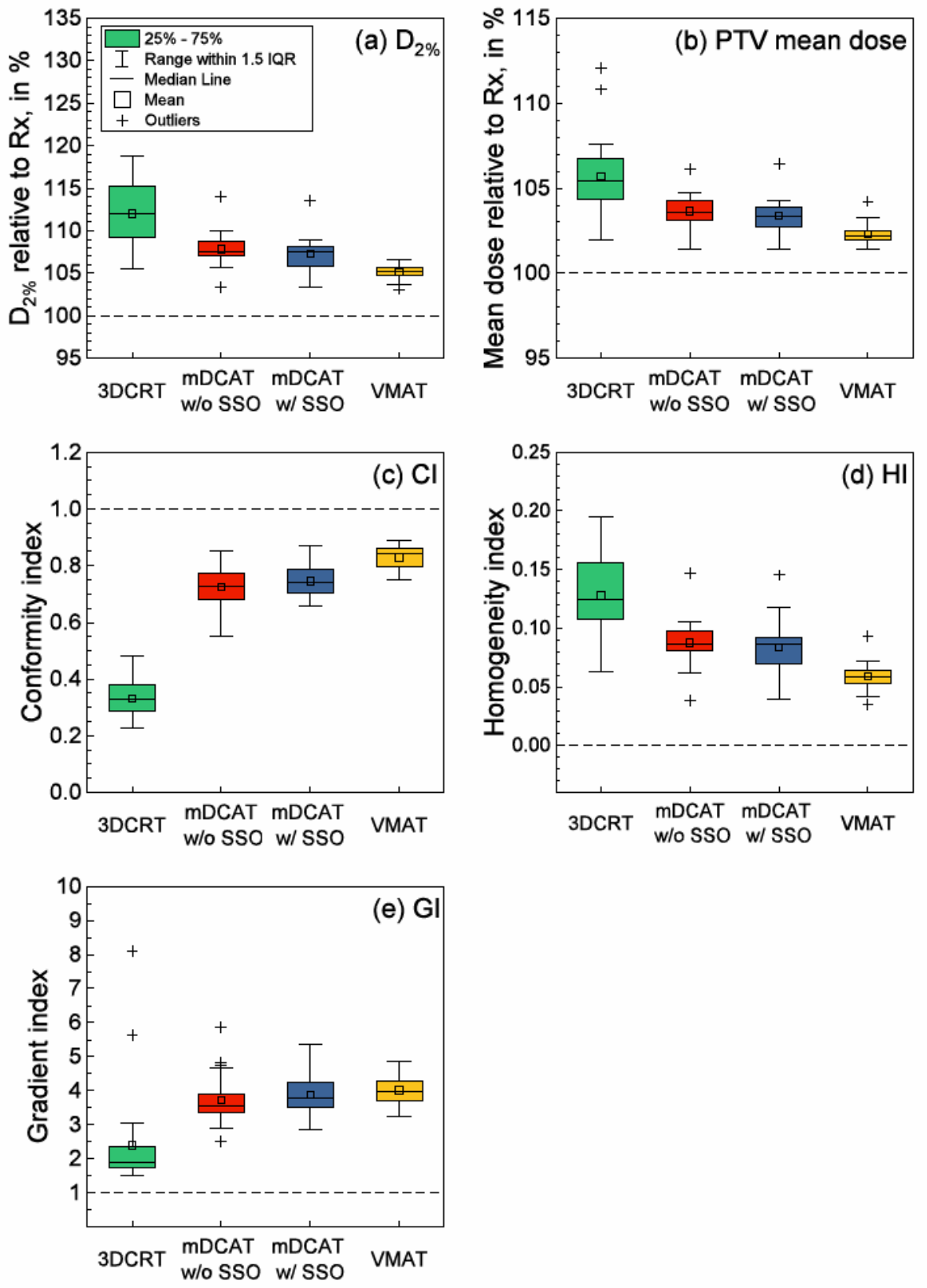

Figure 5.1: Boxplots of the plan quality metrics for 3DCRT, mDCAT without SSO, mDCAT with SSO and VMAT for the twenty palliative spine patients included in this study. The treatment plans were normalized such that for the PTV, $\mathrm{D}_{95 \%}=$ $\mathrm{Rx}$. The ideal value for each metric is represented by the dashed line. The $\mathrm{Rx}$ is 2000 cGy in 5 fractions. 

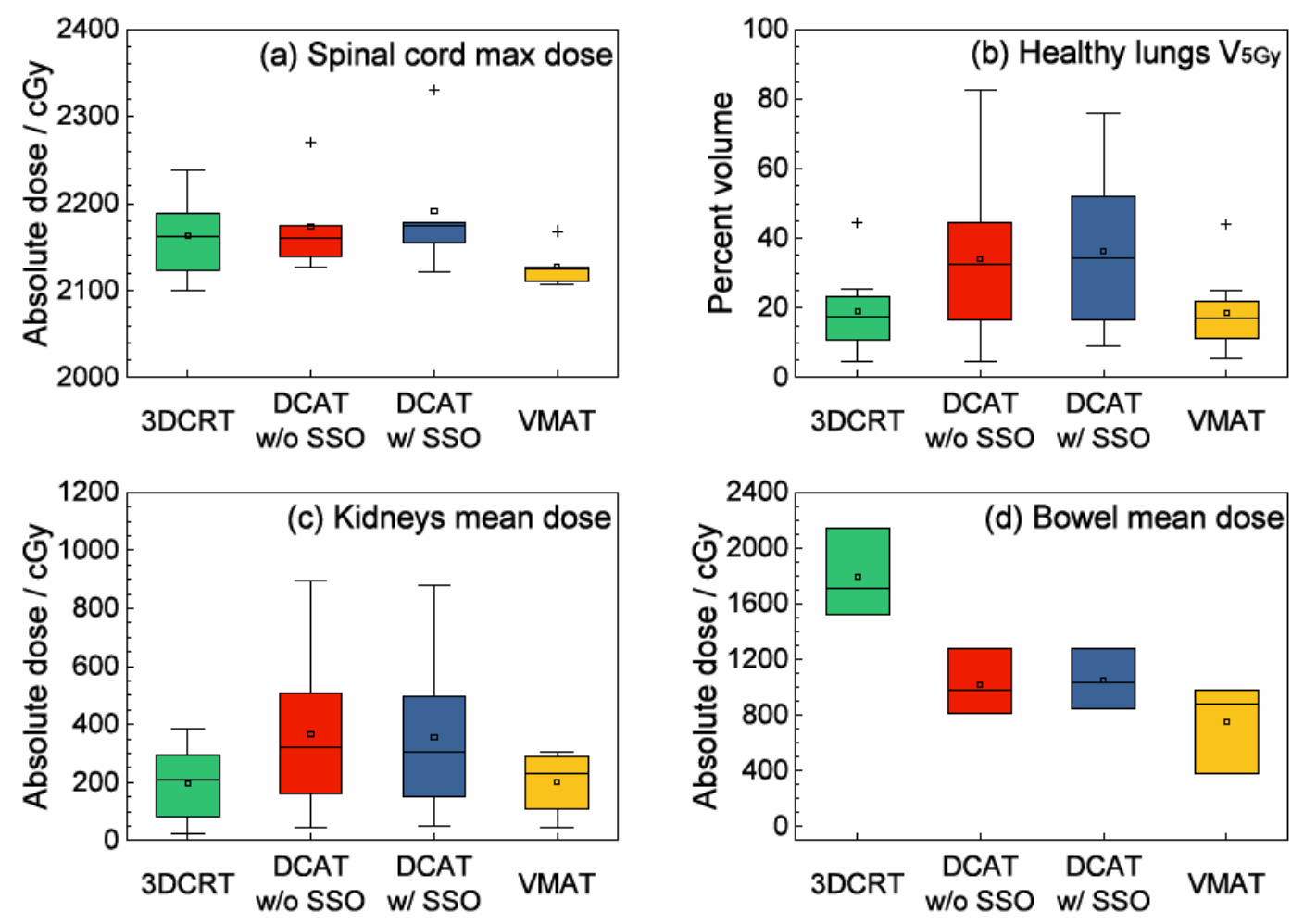

Figure 5.2: For the twenty five palliative spine patients included in this study, the figure shows boxplots of the dose to one of the sensitive OARs surrounding the PTV in different sections of the spine: (a) cervical, (b) thoracic, (c) lumbar, and, (d) sacral. The treatment plans were normalized such that $\mathrm{D}_{95 \%}$ of the PTV $=\mathrm{Rx}$. The $\mathrm{Rx}$ is $2000 \mathrm{cGy}$ to the spine metastasis in 5 fractions.

Table 5.1: Average values of the plan quality metrics and their standard deviations for the 3DCRT, mDCAT (without and with SSO) and VMAT plans of the twenty five palliative spine patients. The treatment plans were normalized such that for the PTV, $\mathrm{D}_{95 \%}=\mathrm{Rx}$.

\begin{tabular}{|r|l|l|l|l|l|}
\hline & $\mathrm{D}_{2 \%}$ & $\begin{array}{l}\text { PTV } \\
\text { mean dose }\end{array}$ & CI & HI & GI \\
\hline Ideal value & $100 \%$ of $\mathrm{Rx}$ & $100 \%$ of $\mathrm{Rx}$ & 1.00 & 0.00 & 1.0 \\
\hline 3DCRT & $112 \pm 4 \%$ & $106 \pm 2 \%$ & $0.33 \pm 0.07$ & $0.13 \pm 0.09$ & $2.4 \pm 1.5$ \\
mDCAT w/o SSO & $108 \pm 2 \%$ & $104 \pm 1 \%$ & $0.72 \pm 0.07$ & $0.09 \pm 0.02$ & $3.7 \pm 0.7$ \\
mDCAT w/ SSO & $107 \pm 2 \%$ & $103 \pm 1 \%$ & $0.75 \pm 0.06$ & $0.08 \pm 0.02$ & $3.9 \pm 0.6$ \\
VMAT & $105 \pm 1 \%$ & $102 \pm 1 \%$ & $0.83 \pm 0.04$ & $0.06 \pm 0.01$ & $4.0 \pm 0.4$ \\
\hline
\end{tabular}



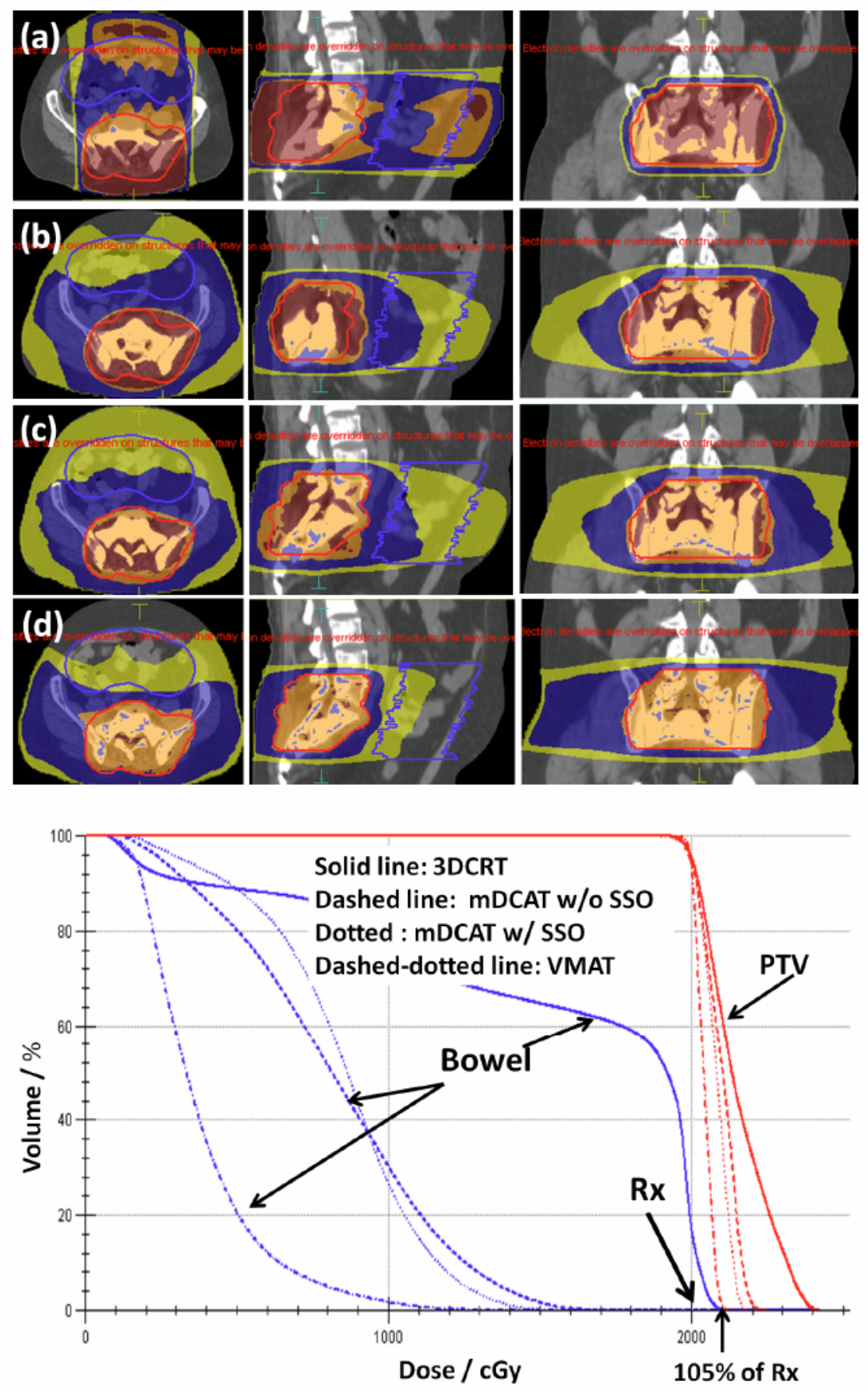

Figure 5.3: For an example palliative spine patient, the figure shows the dose distributions in the axial (left), sagittal (middle) and coronal (right) CT views for (a) 3DCRT, (b) mDCAT without SSO, (c) mDCAT with SSO, and, (d) VMAT plans. The color-washes represent the following percent of the Rx: $50 \%$ (blue), $100 \%$ (orange) and $105 \%$ (brown). The bottom panel shows the corresponding DVHs for the PTV (red) and the bowel (blue). The Rx is 2000 cGy in 5 fractions. 


\subsection{Plan quality for lung SBRT}

The results of comparing the plan quality for mDCAT without and with SSO versus VMAT for the twelve lung SBRT patients are shown in Table 5.2 and Figure 5.4. Table 5.2 shows the average plan quality metrics and their standard deviations, and Figure 5.4 shows boxplots of the plan quality metrics. 3DCRT was not included in the evaluation since 3DCRT is not used for lung SBRT cases when arc delivery is available. For small lung SBRT, doses up to $125 \%$ of Rx inside the GTV are acceptable and clinically desirable $14,20,27,77$ because of the small size of the GTV and that it does not involve functional normal tissues. Therefore the HI is irrelevant and was not included in the comparison of mDCAT versus VMAT plans.

From Table 5.2 and Figure 5.4, the $\mathrm{D}_{2 \%}$ and mean dose to the PTV for mDCAT (without and with SSO) versus VMAT were very comparable. The PTV dose conformity (CI) for mDCAT without SSO was worse compared to that for VMAT. With the addition of SSO to mDCAT, the CI was improved, and it became comparable to that for VMAT. The dose gradient outside the PTV (GI) was slightly worse for VMAT and mDCAT with SSO compared to mDCAT without SSO. VMAT and mDCAT (without and with SSO) offered very comparable sparing of the healthy lung volume, with $\mathrm{V}_{20 G y}$ below the tolerance value for both techniques. The dose distributions and the DVHs for the GTV, PTV and healthy lungs for the mDCAT and VMAT plans for an example lung SBRT patient are shown in Figure 5.5. The DVHs for the GTV were shown to demonstrate that the hotspots of the treatment plans were inside the gross disease.

Overall, for small targets such as those in the lung SBRT cases, the plan quality achievable using mDCAT (without and with $\mathrm{SSO}$ ) is comparable to that using VMAT, except for the PTV dose conformity, which is slightly worse for mDCAT. Adding SSO 
improves the dose conformality compared to mDCAT without SSO.

Table 5.2: Same as in Table 5.1, but for the twelve lung SBRT patients.

\begin{tabular}{|r|l|l|l|l|l|}
\hline & $\mathrm{D}_{2 \%}{ }^{*}$ & $\begin{array}{l}\text { PTV } \\
\text { mean dose }\end{array}$ & CI & GI & $\begin{array}{l}\text { Healthy lungs } \\
\mathrm{V}_{20 G y}\end{array}$ \\
\hline Ideal value & $100 \%$ of $\mathrm{Rx}$ & $100 \%$ of $\mathrm{Rx}$ & 1.00 & 1.00 & $0.0 \%$ \\
\hline mDCAT w/o SSO & $125 \pm 6$ & $110 \pm 2$ & $0.67 \pm 0.06$ & $5.7 \pm 0.9$ & $5.7 \pm 2.4$ \\
mDCAT w/ SSO & $124 \pm 5$ & $109 \pm 2$ & $0.76 \pm 0.06$ & $6.8 \pm 1.2$ & $6.0 \pm 2.4$ \\
VMAT & $126 \pm 5$ & $109 \pm 2$ & $0.83 \pm 0.03$ & $6.9 \pm 1.8$ & $5.3 \pm 1.7$ \\
\hline
\end{tabular}

*Doses up to $125 \%$ of the Rx at the centre of the GTV are allowed and desirable for lung SBRT cases 

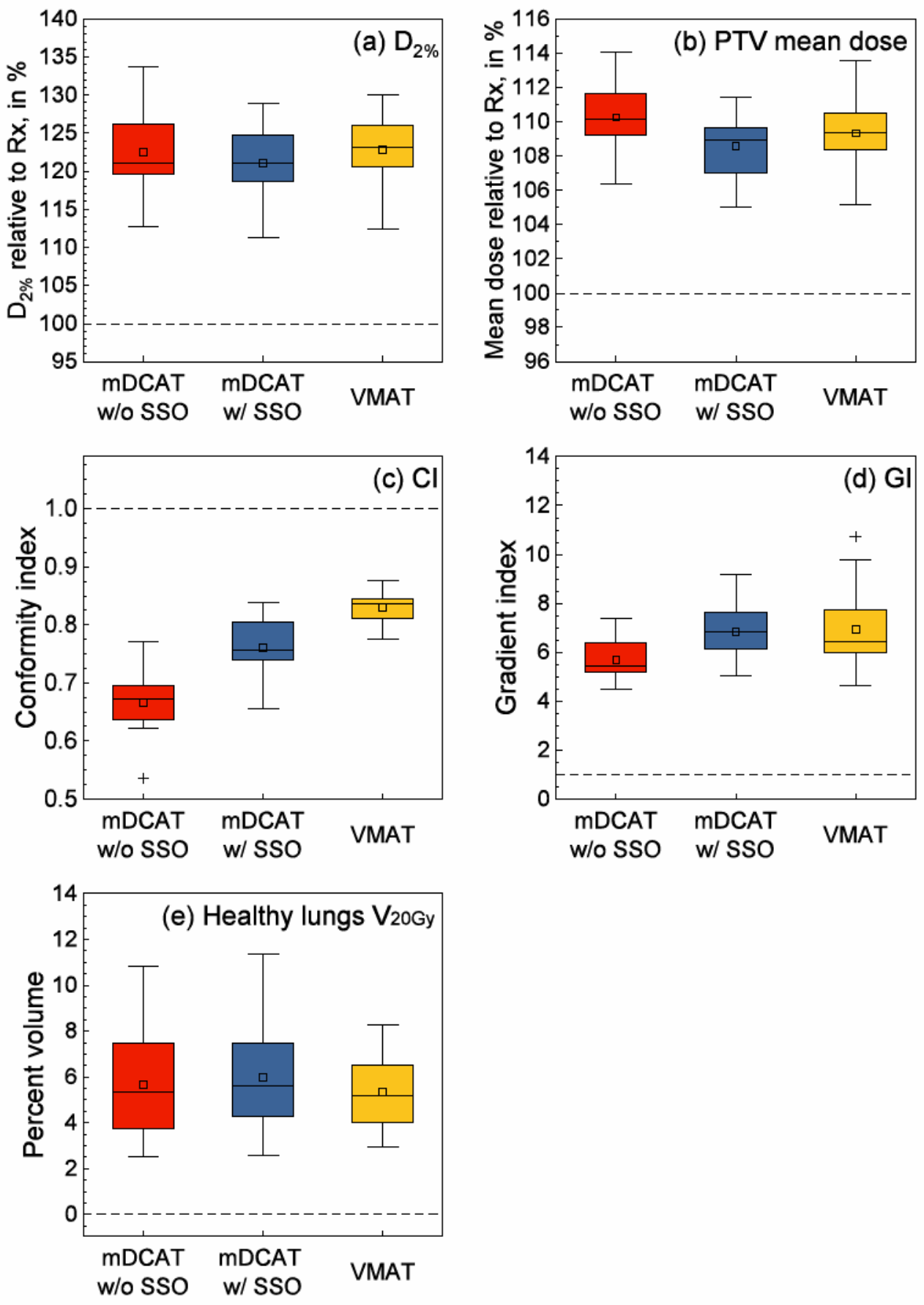

Figure 5.4: Same as in Figure 5.1, but for the twelve lung SBRT patients included in this study. The Rx either is 6000 cGy in 8 fractions or 5400 cGy in 3 fractions. 

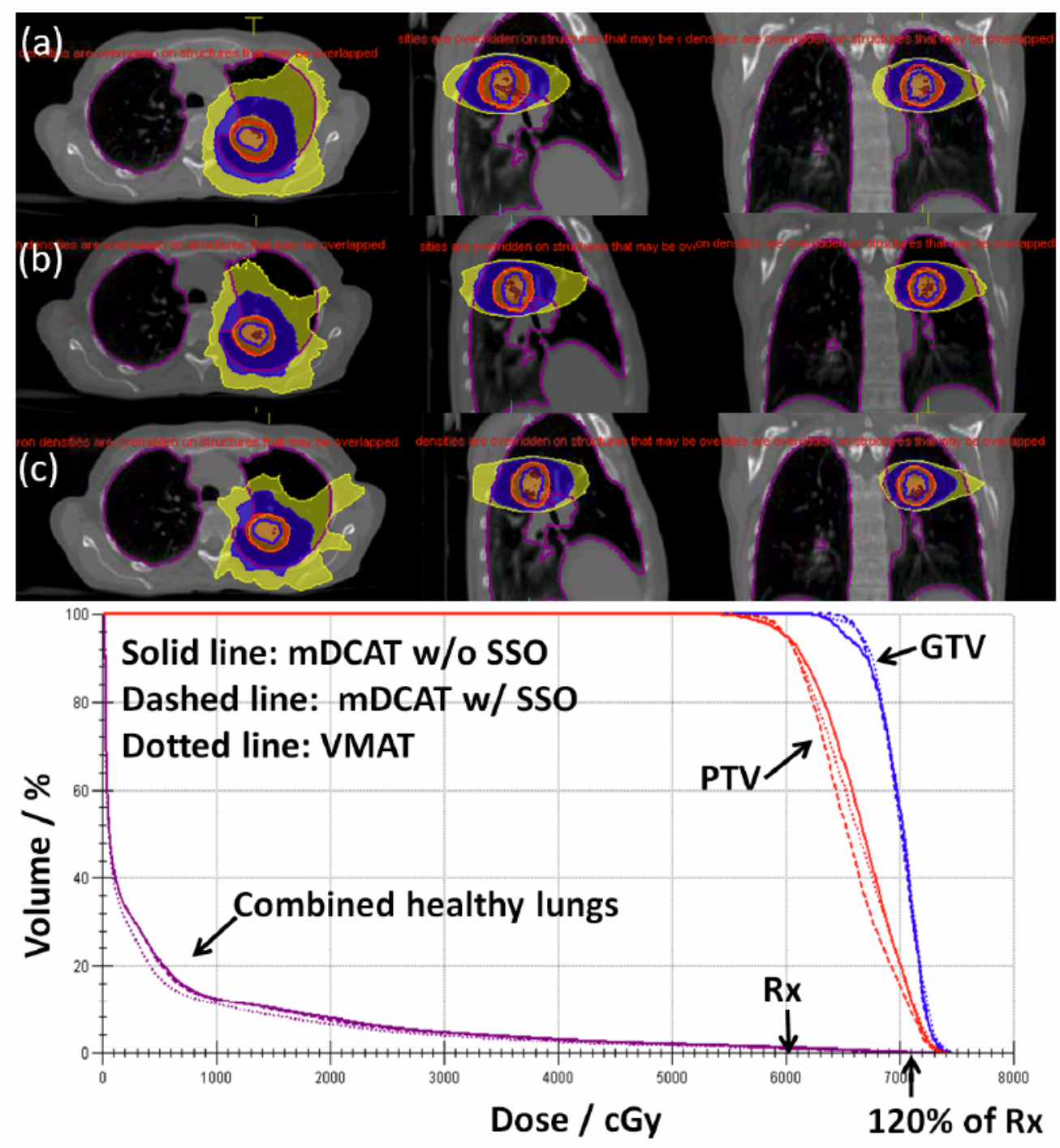

Figure 5.5: For an example lung SBRT patient, the figure shows the dose distributions in the axial (left), sagittal (middle) and coronal (right) CT views for (a) mDCAT without SSO, (b) mDCAT with SSO, and, (c) VMAT plans. The colorwashes represent the following percent of the Rx: $33 \%$ or $20 \mathrm{~Gy}$ (yellow), $50 \%$ (blue) and $100 \%$ (orange) and $120 \%$ (brown). The bottom panel shows the corresponding DVHs for the GTV (blue), PTV (red) and the healthy lungs (purple). The Rx is 6000 cGy in 8 fractions. 


\subsection{Plan quality for brain}

The results of comparing the plan quality for mDCAT without and with SSO versus VMAT for the twelve brain patients are shown in Table 5.3 and Figure 5.6. Table 5.3 shows the average plan quality metrics and their standard deviations, and Figure 5.6 shows boxplots of the plan quality metrics. For reasons similar to those for lung SBRT, 3DCRT was not considered, and the HI was not included as a metric.

From Table 5.3 and Figure 5.6 , the $\mathrm{D}_{2 \%}$ for the PTV was slightly better for mDCAT (without and with SSO) compared to that for VMAT. The PTV mean dose achievable with mDCAT (without and with SSO) was very comparable to that achievable with VMAT. The PTV dose conformity (CI) for mDCAT without SSO was slightly worse than for VMAT. Adding SSO to mDCAT generated a more conformal distribution versus mDCAT without SSO, and a comparable conformity to that with VMAT. The dose gradient outside the PTV (GI) was very comparable for VMAT and mDCAT with SSO. In terms of dose to the OARs, for cases where the PTV was away from the OARs, mDCAT (without and with SSO) was very comparable to VMAT in sparing of the optic structures and the brainstem. However, for cases in which the PTV was surrounded by, adjacent to, or overlapping with the OAR, mDCAT without SSO offered substantially worse OAR sparing compared to VMAT. Adding SSO to mDCAT for those challenging cases substantially reduced the doses to the OARs and produced sparing comparable to that for VMAT. As an example, Figure 5.7 shows the dose distributions and the DVHs for the GTV, PTV, optic chiasm and brainstem for the mDCAT and VMAT plans for a brain patient with a PTV close to the OARs. In this example, the maximum dose to the brainstem was $22.5 \%, 10.8 \%$ and $10.2 \%$ of the $\mathrm{Rx}$ for mDCAT without SSO, mDCAT with SSO and VMAT plans, respectively, and the maximum dose to the optic 
structure was $31.2 \%, 15.0 \%$ and $13.7 \%$ of the $\mathrm{Rx}$, respectively.

Overall for small targets in the brain, when the target is away from the OARs, mDCAT (without or with SSO) and VMAT offered comparable sparing of the OAR. However, when the target is close to the OARs, mDCAT with SSO and VMAT offered substantially better OAR sparing compared to mDCAT without SSO.

Table 5.3: Same as in Table 5.1, but for the twelve brain patients.

\begin{tabular}{|r|l|l|l|l|}
\hline & $\mathrm{D}_{2 \%}{ }^{*}$ & $\begin{array}{l}\text { PTV } \\
\text { mean dose }\end{array}$ & CI & GI \\
\hline Ideal value & $100 \%$ of $\mathrm{Rx}$ & $100 \%$ of $\mathrm{Rx}$ & 1.00 & 1.00 \\
\hline mDCAT w/o SSO & $113 \pm 2$ & $107 \pm 1$ & $0.70 \pm 0.04$ & $4.2 \pm 1.0$ \\
mDCAT w/ SSO & $112 \pm 3$ & $106 \pm 2$ & $0.76 \pm 0.08$ & $5.2 \pm 1.3$ \\
VMAT & $115 \pm 5$ & $107 \pm 3$ & $0.85 \pm 0.09$ & $5.8 \pm 2.1$ \\
\hline
\end{tabular}

${ }^{*}$ Doses higher than $107 \%$ of the Rx at the centre of the GTV are allowed for brain cases with small PTVs 

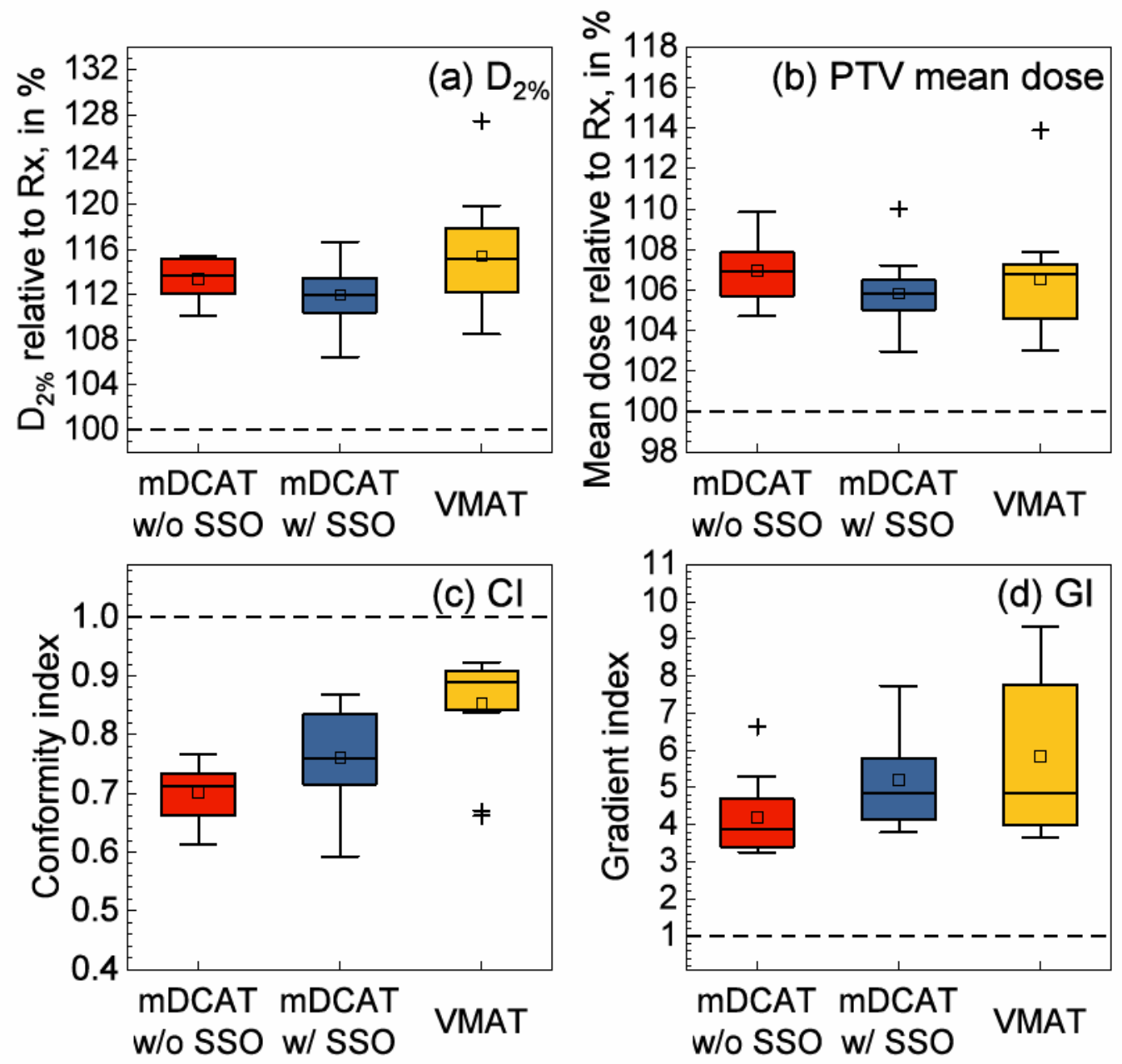

Figure 5.6: Same as in Figure 5.1, but for the twelve brain patients included in this study. The Rx dose is 2400 cGy in 3 fractions. 

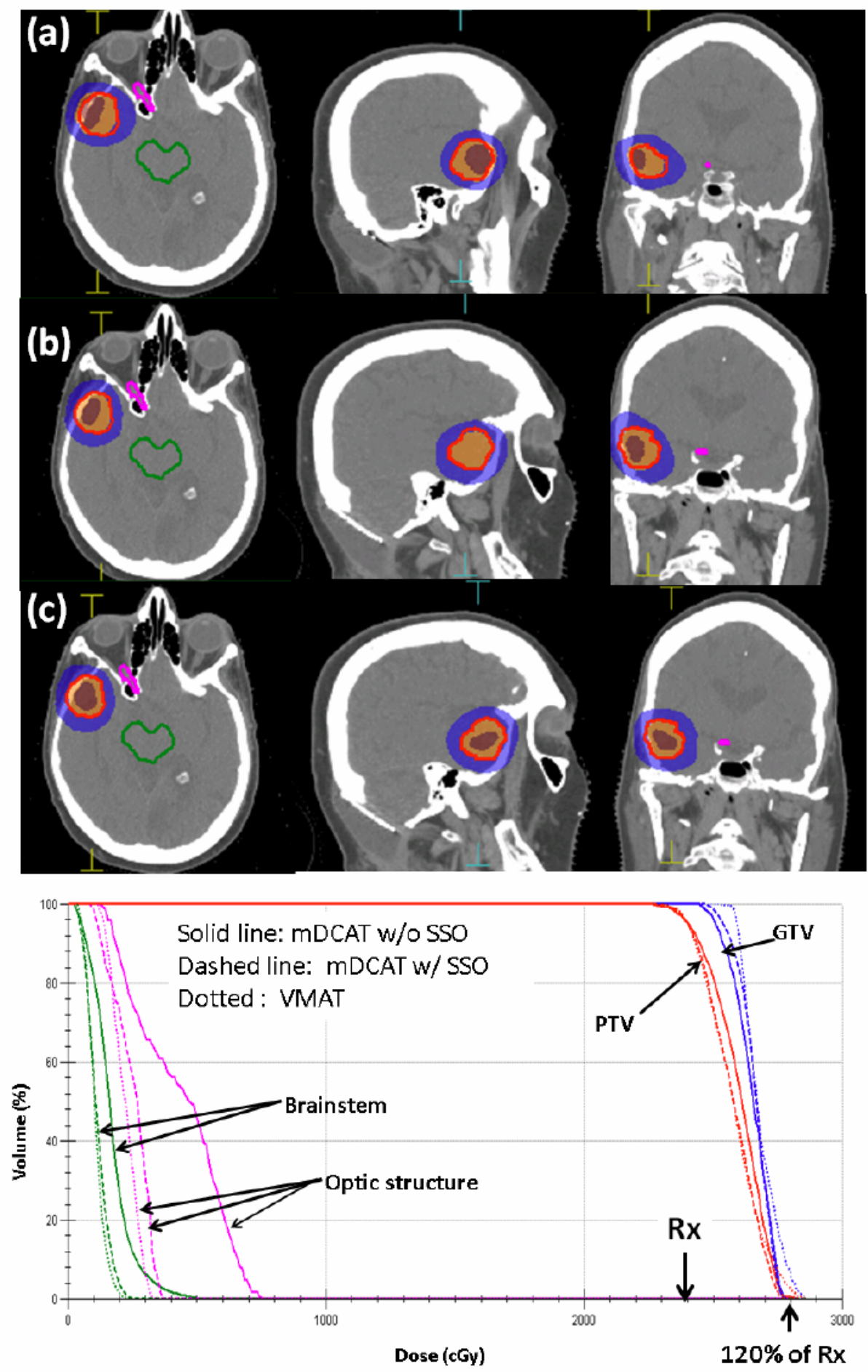

Figure 5.7: For an example brain patient, the figure shows the dose distributions in the axial (left), sagittal (middle) and coronal (right) CT views for (a) mDCAT without SSO, (b) mDCAT with SSO, and, (c) VMAT plans. The color-washes represent the following percent of the Rx: $50 \%$ (blue) and $100 \%$ (orange) and $110 \%$ (brown). The bottom panel shows the corresponding DVHs for the GTV (blue), PTV (red), optic structures (purple) and brainstem (green). The Rx is 2400 cGy in 3 fractions. 


\subsection{Summary}

For the palliative spine cases, mDCAT performs midway between the clinically acceptable 3DCRT and VMAT plans based on the dose distributions, DVHs and other plan quality metrics, with slight improvement for mDCAT with SSO versus without SSO. The main advantage of VMAT and mDCAT versus 3DCRT is the target dose conformality that arc deliveries can produce. This translates into lower doses to the surrounding OARs for most VMAT and mDCAT treatment plans. Partial arcs can be used for better sparing of lateral OARs such as kidneys and lungs. For the lung SBRT and brain patients, the plans achievable with VMAT and mDCAT were very comparable except for the dose conformality and the gradient outside the target (for

brain cases only), in which mDCAT without SSO was worse compared to VMAT. The addition of SSO to mDCAT improved the dose conformality and gradient, making the value of these metrics comparable to those for VMAT. 


\section{Chapter 6}

\section{Robustness against machine uncertainties}

A medical LINAC is a complex piece of equipment that requires meticulous testing to ensure that its components are functioning with high degree of accuracy and precision. Daily, monthly and annual LINAC QA programs are implemented in RT departments

following recommendations from guidance documents. ${ }^{94-96}$ These documents provide recommendations on the type of tests to be performed for these multi-component systems, their frequency, and the action levels for the tests.

In the era of 3DCRT, QA procedures were simpler due to the straightforward implementation of the 3DCRT technique. However, with the development of modulated treatments (such as IMRT, VMAT and mDCAT), in which different LINAC components are allowed to move during beam-on time, a more stringent LINAC quality assurance program is typically required. The robustness of the dose distributions from modulated treatments is generally more vulnerable to uncertainties than non-modulated treatments because of the steeper dose gradients. Mu et al ${ }^{97}$ showed, on average, $4 \%$ and $8 \%$ deviations from planned target coverage for simple and complex IMRT plans, respectively, for a systematic error of $1 \mathrm{~mm}$ in MLC leaf positions. A systematic MLC offset as small as $0.3 \mathrm{~mm}$ for VMAT plan delivery was shown to produce a $3 \%$ deviation in the dosimetric end points. ${ }^{98}$

Machine uncertainties can be simplified as either systematic (e.g., a weak MLC 
motor, MLC gain/offset calibration error, systematic calibration offset on other LINAC components) and/or random (e.g., MLC leaves randomly not catching up during the sweeping motion in modulated treatments). In this chapter, the robustness of mDCAT and VMAT plans against machine uncertainties were assessed by artificially introducing small perturbations to the expected MLC leaf positions of the beam segments, and quantifying the dosimetric impact of these small perturbations on plan quality. Robust treatment plans are preferred, and robustness is manifested by minimal change in plan quality metrics when perturbations are introduced, with the technique offering the least changes being the most robust technique against such uncertainties.

\subsection{Modelling machine errors}

MLC positional errors are particularly relevant examples of machine uncertainties due to the complexity and sensitivity of the leaf collimation system. A Python ${ }^{99}$ code was developed to manipulate the plan files in the back-end of the Monaco TPS to digitally introduce extreme and typical positional perturbations to the active MLC leaves ("active" means within the open field of a segment) in mDCAT and VMAT plans. For both the extreme and typical errors, the nature of the errors, both systematic and random, was also explored.

For extreme systematic MLC leaf errors, the magnitude of the errors was taken from the tolerance levels given in guidance documents, ${ }^{94}$ which is $2 \mathrm{~mm}$. The MLC leaves were once opened (i.e., moved away from the target edge) and once closed (i.e., moved towards the target edge) relative their true positions.

For typical errors, the individual MLC leaf errors for a given control point were sampled using the distribution of the aggregate results of the QA program at TOHCC for four Elekta LINACs with Agility MLC assemblies over a period of four months (see 


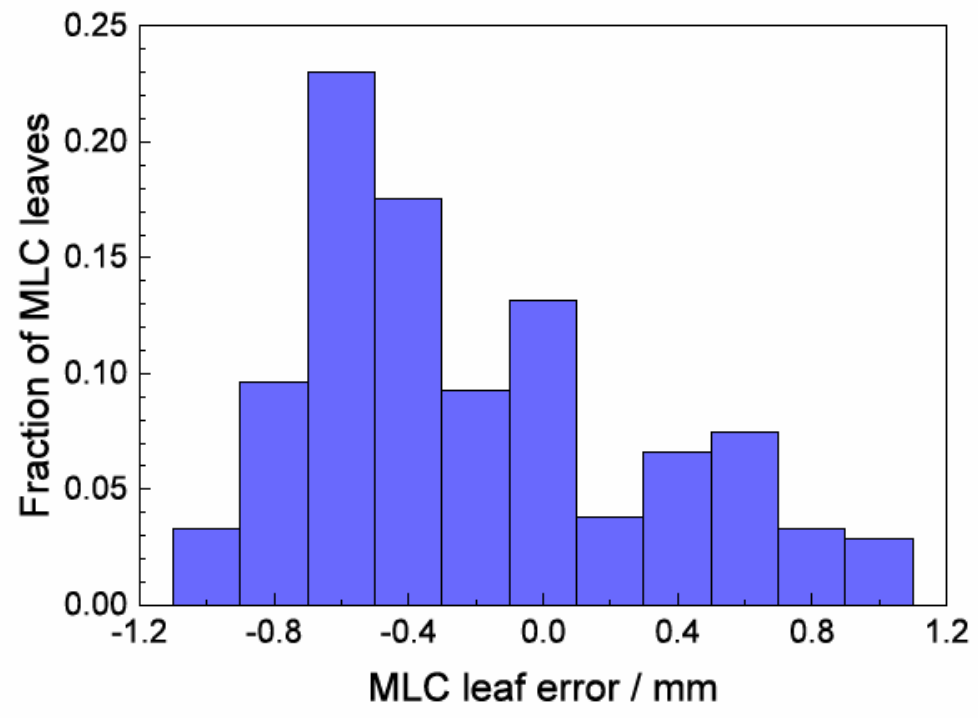

Figure 6.1: MLC offset distribution using the aggregate MLC QA data for four LINACs with Agility MLC assembly at TOHCC.

Figure 6.1). The data in the figure are comparable with those measured previously. ${ }^{64}$ For the systematic type of those typical errors, the same error sampled from the distribution for each MLC leaf was kept throughout the whole treatment. This models the situation where systematic MLC leaf uncertainties (such as a calibration offset), dominate. For the random type of those typical errors, a different error for each leaf was sampled from the same distribution for each control point (starting or ending position of the LINAC components for a beam segment). This models the situation where the dynamic MLC leaf uncertainties (such as leaves not catching up during sweeping motion), dominate.

To evaluate the MLC positional errors specifically during mDCAT and VMAT deliveries, six representative patients from each of the three disease sites (spine, lung and brain) were chosen, and their VMAT and mDCAT plans were delivered, then the LINAC $\log$ files were extracted and analyzed. In these log files, the MLC positional errors from their expected true positions are reported every 40 milliseconds.

Shown in Figures 6.2 and 6.3 are the histograms for the MLC errors during the 


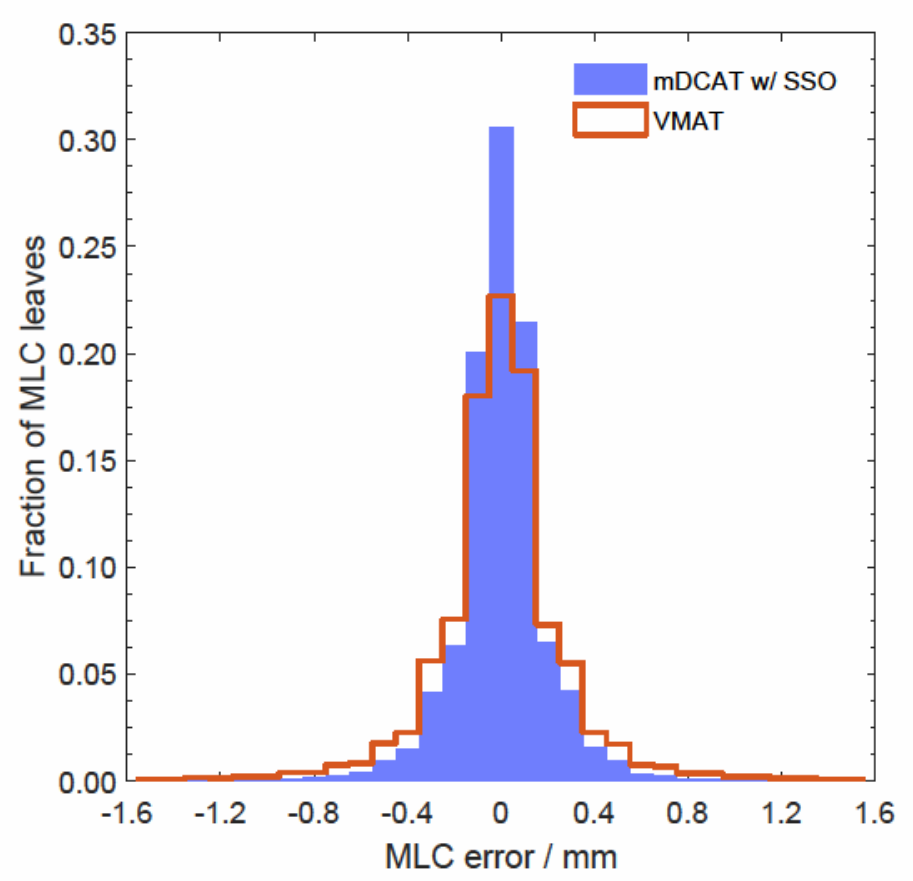

Figure 6.2: Experimental results for the MLC positional errors for the combination of six spine patients, six lung SBRT patients, and six brain patients delivered on an Elekta Infinity LINAC at TOHCC. Data were extracted from machine log files for the mDCAT and VMAT deliveries.

deliveries of the VMAT and mDCAT plans for all patients combined and per disease site (i.e., spine, lung SBRT and brain), respectively. The results confirm that the QA data mostly reflect the actual MLC positional errors for VMAT and mDCAT deliveries. The results also show that the MLC errors in mDCAT deliveries are slightly less than those for VMAT, as reflected in the width of the spread of the distributions for mDCAT deliveries relative to that for the corresponding VMAT deliveries, because mDCAT involves less MLC movement and thus it is less demanding on the performance of the LINAC components.

Computationally, the different types of MLC errors discussed above were artificially introduced into the mDCAT and VMAT plans of the twenty five palliative spine, twelve lung SBRT, and twelve brain patients. After introducing the MLC errors, the plans were re-calculated without re-optimizing to retain the original beam segment details, 

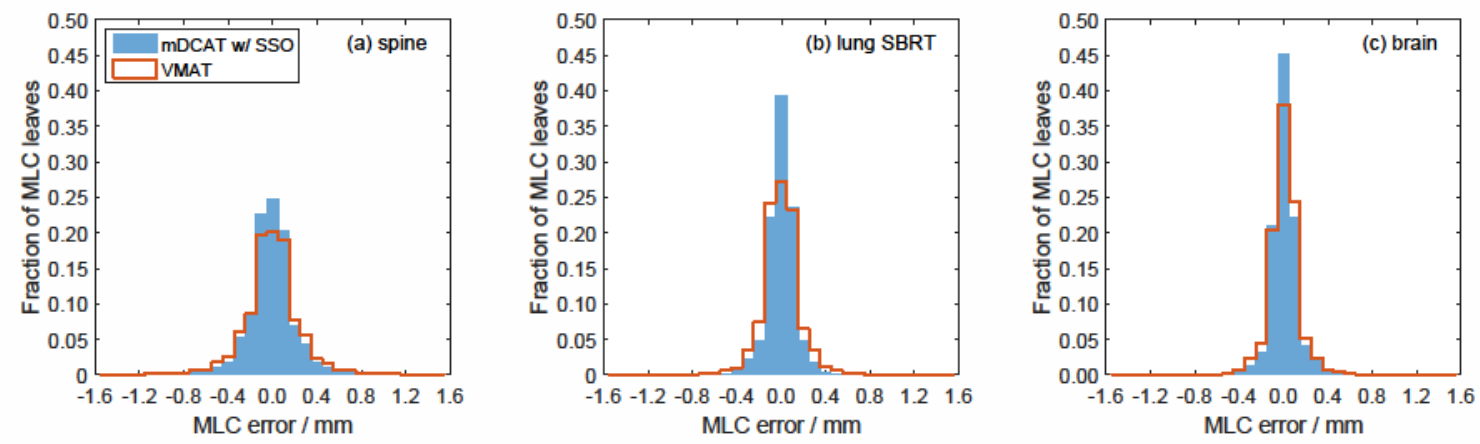

Figure 6.3: Same as in Figure 6.2 but for MLC errors plotted per disease site.

and thus isolate the effect of the modelled MLC errors. The original plans and the plans with the introduced errors were calculated using the same calculation parameters.

A reasonable assumption is that the introduced MLC errors represent the cumulative effect of setup margins (patient setup variation and machine uncertainties), and hence to evaluate the plan quality metrics after the introduction of the errors, the targets used were: CTV for the spine, ITV for the lung SBRT, and GTV for the brain cases (refer to Figure 1.4 for the target volume and margin definitions).

The tables and graphs in this chapter summarize the results quantitatively. This is followed by a qualitative description of those results and their clinical relevance in the body of the text.

\subsection{Dosimetric impact of extreme MLC errors}

The results of the dosimetric impact of modelling extreme systematic MLC errors are shown in Table 6.1 and Figures 6.4, 6.5 and 6.6. Table 6.1 shows the average changes in the plan quality metrics and their standard deviations, and Figures $6.4,6.5$ and 6.6 show the corresponding boxplots for the changes in the plan quality metrics. Good robustness against a modelled error is manifested in the metrics of plan quality in two ways: (1) 
the mean values of the metrics remain close to their values for the original plans without the modelled error (represented by the dashed lines in the panels), and, (2) the spread around the mean values of the change in the metrics are small (represented by a small size of the boxes in the boxplots, and by shorter whiskers and less outliers).

For the spine cases with extreme systematic MLC errors (Figure 6.3 and the first data column of Table 6.1), after opening the MLC leaves by $2 \mathrm{~mm}$, the changes in $\mathrm{D}_{95 \%}, \mathrm{D}_{2 \%}$ and mean dose to the CTV was substantially better for mDCAT (without and with SSO) compared to VMAT. The changes for these three plan quality metrics were positive, indicating that the plans produced after opening the MLC leaves were hotter relative to the base plans (which are the treatment plans without the errors). The $25 \%-75 \%$ boxes for the changes in the CTV $\mathrm{D}_{95 \%}, \mathrm{D}_{2 \%}$ and mean doses were very narrow and very close to the values of the metrics from the base plans for both mDCAT without and with SSO, which implies that the CTV dose coverage and the high dose volumes were almost unaffected by the introduction of the extreme systematic MLC errors. For mDCAT without SSO, the MLC leaves are shaped around the target edge, and opening the MLC leaves by $2 \mathrm{~mm}$ did not affect the coverage for these large targets. Even with the added modulation by using SSO, mDCAT plans demonstrated comparable robustness against these errors between mDCAT without SSO and with SSO. For VMAT, the complex shapes of the beam segments and the typical shadowing of the MLC leaves over the target as part of its modulation (see Figure 1.8 for the example VMAT beam segment) made it more vulnerable to these extreme cases.

The change in the dose conformality (CI) for mDCAT (without and with SSO) was slightly better compared to VMAT. The changes in CI for both VMAT and mDCAT were negative, which means that the produced dose distributions were less conformal to the target compared to the base plans. The reason is that, by opening the MLC 
leaves from their true positions, more non-target tissues were irradiated, and the ratio between the target volume and the volume receiving the $\mathrm{Rx}$ decreased, which reduced the CI (see Equation 2.1) compared to the base plans. For the changes in the CI value, although the average values for VMAT and mDCAT were comparable, the spread was more for VMAT. The changes in the HI for mDCAT and VMAT were comparable. The changes in the gradient outside the CTV (GI) were better for mDCAT (without and with SSO) versus that with VMAT. The ratio between the $50 \%$ isodose volume and $100 \%$ isodose volume (Equation 2.3) after opening the MLC leaves was almost unchanged for mDCAT, and this ratio was almost doubled for VMAT.

When MLC leaves were closed by $2 \mathrm{~mm}$ (not shown in the table or boxplots), the percent dose errors were of the same magnitude as those with opening the MLC leaves, but with opposite dose changes (i.e., the dose distributions were colder versus the base plans). For mDCAT, closing the MLC leaves by $2 \mathrm{~mm}$ did not cause underdosage to the CTV for the spine patients since the introduced error was smaller than the setup margin used during treatment planning (i.e., $5 \mathrm{~mm}$ ). On the other hand, by the nature of modulation in the VMAT plans, there were MLC leaves shadowing the CTV, and thus closing the MLC leaves caused substantial reduction in the CTV dose coverage, in particular the $\mathrm{D}_{95 \%}$. For all the plan quality metrics (except for the HI), the changes were marginally worse for mDCAT with SSO compared to those for mDCAT without SSO. The modulation for mDCAT with SSO for large targets (such as for palliative spine cases) is only around the periphery (as concluded earlier in Chapter 3), and this moderate modulation did not degrade the robustness of mDCAT with SSO (compared to mDCAT without SSO) against machine uncertainties for large targets.

For the lung SBRT cases with extreme systematic MLC errors (Figure 6.5 and the second data column of Table 6.1), the magnitude of the changes for the plan quality 
metrics after opening the MLC leaves was higher compared to the changes in the metrics for the spine cases discussed earlier. The reason is that the introduced $2 \mathrm{~mm}$ error made a larger fraction of the field for smaller targets and smaller beam segments compared to larger targets and larger beam segments. For mDCAT plans, the effect of turning SSO ON or OFF on the changes in ITV dose coverage $\left(\mathrm{D}_{95 \%}, \mathrm{D}_{2 \%}\right.$ and mean dose) was more pronounced for lung SBRT cases compared to the spine cases. This is because the lung SBRT targets have smaller segments compared to the spine targets, and thus the modulation with mDCAT is relatively more obvious (as concluded in Chapter 3). However, the changes for the ITV $\mathrm{D}_{95 \%}, \mathrm{D}_{2 \%}$ and mean dose were still better (i.e., the plans are more robust) versus VMAT. The changes in CI were slightly better for mDCAT compared to VMAT. The GI changes were comparable between VMAT and mDCAT.

For the brain cases with extreme systematic MLC errors (Figure 6.6 and the last data column of Table 6.1), similar behaviour to that for the lung SBRT cases was observed. The brain and lung cases included in this study had small PTVs (less than 50 $\mathrm{cm}^{3}$ and $20 \mathrm{~cm}^{3}$ for lung SBRT and brain, respectively). The effect on dose distributions for either mDCAT or VMAT plans was comparable for similar target sizes after the introduction of extreme systematic MLC errors.

Collectively from Figures 6.4, 6.5 and 6.6 for the three disease sites, it can be concluded that for all the quality metrics (except for the HI for spine cases), mDCAT (without and with SSO) was more robust compared to VMAT, manifested as a smaller deviation from the base plans, narrower $25 \%$ to $75 \%$ boxes, and less extreme changes for some individual patients (whiskers and outliers).

In summary, the dose distributions produced with mDCAT plans were more robust against extreme systematic error for both large and small targets compared to VMAT, 
mainly because of the high degree of modulation that VMAT has. For large targets (such as for palliative spine), the effect on robustness of the moderate modulation for mDCAT with SSO was marginally worse because the modulation is just around the target edges. For smaller targets (such as for lung SBRT and brain), the magnitude of the changes in the plan quality is magnified versus that with the large targets because the errors make a larger fraction of the beam segments. The addition of SSO in mDCAT for these small targets increased the changes in the metrics (i.e., less robust) versus that without SSO, but the values are still lower (i.e., more robust) for mDCAT with SSO compared to VMAT. 
Table 6.1: Average changes in the plan quality metrics and their standard deviations after introducing intentional extreme systematic MLC errors (opening $2 \mathrm{~mm}$ from true positions) to the mDCAT and VMAT plans of the twenty five palliative spine, twelve lung SBRT and twelve brain patients included in this study. The target coverage metrics are evaluated for the CTV in the spine cases, the ITV in the lung SBRT cases, and the GTV in the brain cases.

\begin{tabular}{|c|c|c|c|c|}
\hline \multirow{2}{*}{ Metric } & \multirow{2}{*}{ Technique } & \multicolumn{3}{|c|}{ Change in metric } \\
\hline & & Spine & Lung SBRT & Brain \\
\hline \multirow{3}{*}{$\begin{array}{l}\mathrm{D}_{95 \%} \\
\text { relative to } \mathrm{Rx} \text {, } \\
\text { in } \%\end{array}$} & mDCAT w/o SSO & $0.5 \pm 0.2$ & $1.9 \pm 0.8$ & $1.5 \pm 0.8$ \\
\hline & mDCAT w/ SSO & $0.7 \overline{+} 0.3$ & $3.0+1.1$ & $1.8+1.5$ \\
\hline & VMAT & $4.0 \pm 1.9$ & $6.5 \pm 1.8$ & $4.7 \pm 2.6$ \\
\hline \multirow{3}{*}{$\begin{array}{l}\mathrm{D}_{2 \%} \\
\text { relative to } \mathrm{Rx} \text {, } \\
\text { in } \%\end{array}$} & mDCAT w/o SSO & $0.5 \pm 0.1$ & $1.0 \pm 0.3$ & $0.9 \pm 0.5$ \\
\hline & mDCAT w/ SSO & $0.6 \pm 0.1$ & $1.3 \pm 0.3$ & $1.0 \pm 0.5$ \\
\hline & VMAT & $5.1 \pm 2.6$ & $4.5 \pm 3.5$ & $3.7 \pm 2.4$ \\
\hline \multirow{3}{*}{$\begin{array}{l}\text { Mean dose } \\
\text { relative to } \mathrm{Rx} \text {, } \\
\text { in } \%\end{array}$} & mDCAT w/o SSO & $0.5 \pm 0.1$ & $1.3 \pm 0.3$ & $0.5 \pm 1.1$ \\
\hline & mDCAT w/ SSO & $0.6 \pm 0.2$ & $1.9 \pm 0.5$ & $0.7 \pm 1.4$ \\
\hline & VMAT & $4.3 \pm 2.1$ & $5.9 \pm 2.1$ & $2.7 \pm 4.0$ \\
\hline \multirow{3}{*}{ CI } & mDCAT w/o SSO & $-0.03 \pm 0.01$ & $-0.04 \pm 0.01$ & $-0.04 \pm 0.02$ \\
\hline & mDCAT w/ SSO & $-0.04 \pm 0.02$ & $-0.04 \pm 0.01$ & $-0.05 \pm 0.02$ \\
\hline & VMAT & $-0.07 \pm 0.08$ & $-0.07 \pm 0.02$ & $-0.05 \pm 0.03$ \\
\hline \multirow[t]{2}{*}{ HI } & $\begin{array}{l}\text { mDCAT w/o SSO } \\
\text { mDCAT w/SSO }\end{array}$ & $\begin{array}{l}5.8 \pm 2.7 \\
5.4 \pm 2.7\end{array}$ & see footnote & see footnote \\
\hline & VMAT & $5.7 \pm 1.7$ & & \\
\hline \multirow{3}{*}{ GI } & mDCAT w/o SSO & $-0.2 \pm 0.1$ & $-0.3 \pm 0.2$ & $-0.4 \pm 0.3$ \\
\hline & mDCAT w/ SSO & $-0.3 \pm 0.2$ & $-0.5 \pm 0.2$ & $-0.6 \pm 0.3$ \\
\hline & VMAT & $-1.6 \pm 2.3$ & $-0.8 \pm 0.4$ & $-0.9 \pm 0.7$ \\
\hline
\end{tabular}

HI not evaluated for stereotactic treatments. See Section 2.7 for discussion. 

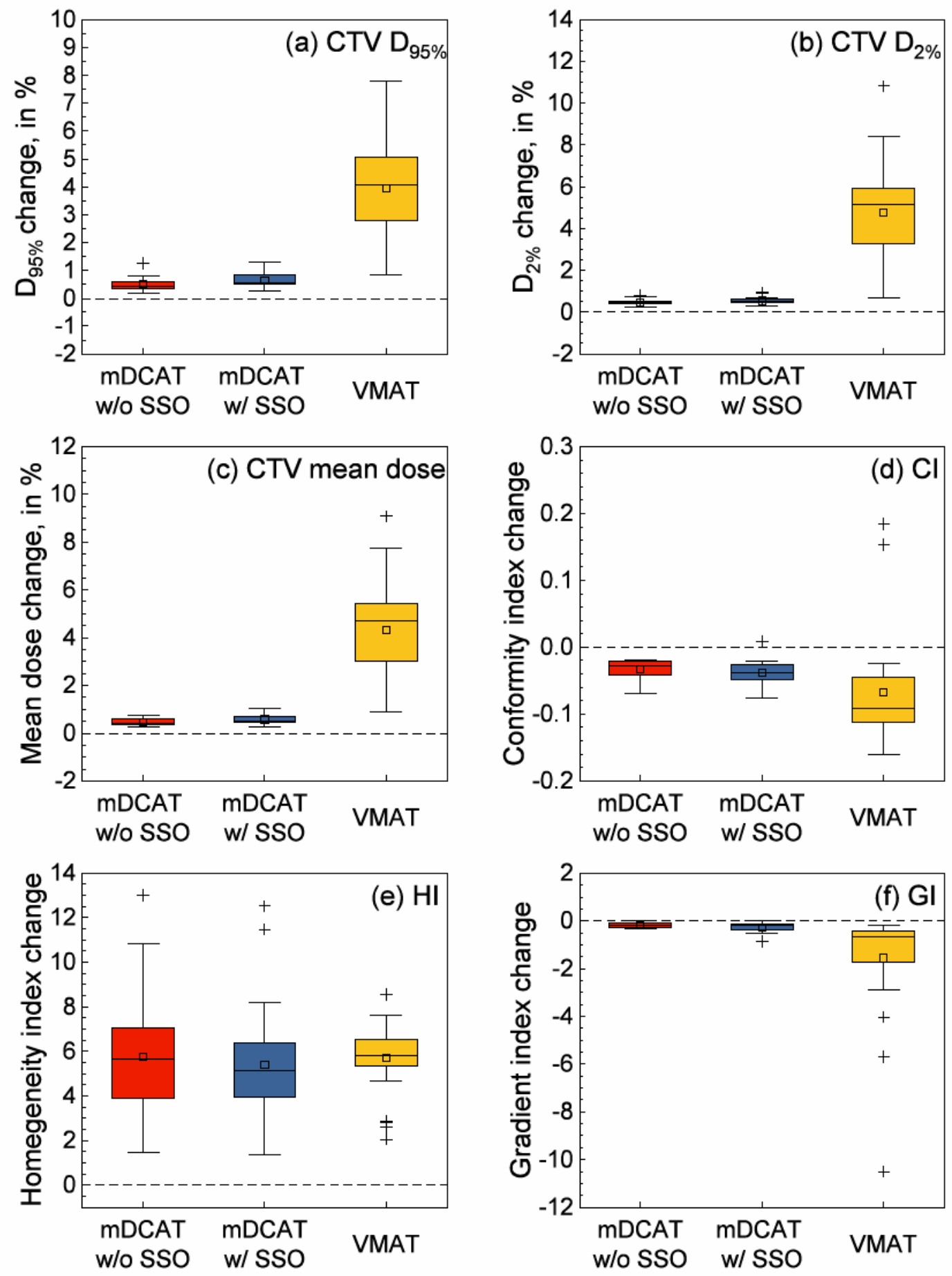

Figure 6.4: Boxplots of the changes in the values of plan quality metrics for the twenty five palliative spine patients included in this study after "opening" the MLC leaves by $2 \mathrm{~mm}$ from their true positions. "Opening" means moving the MLC leaves away from the target edge. 

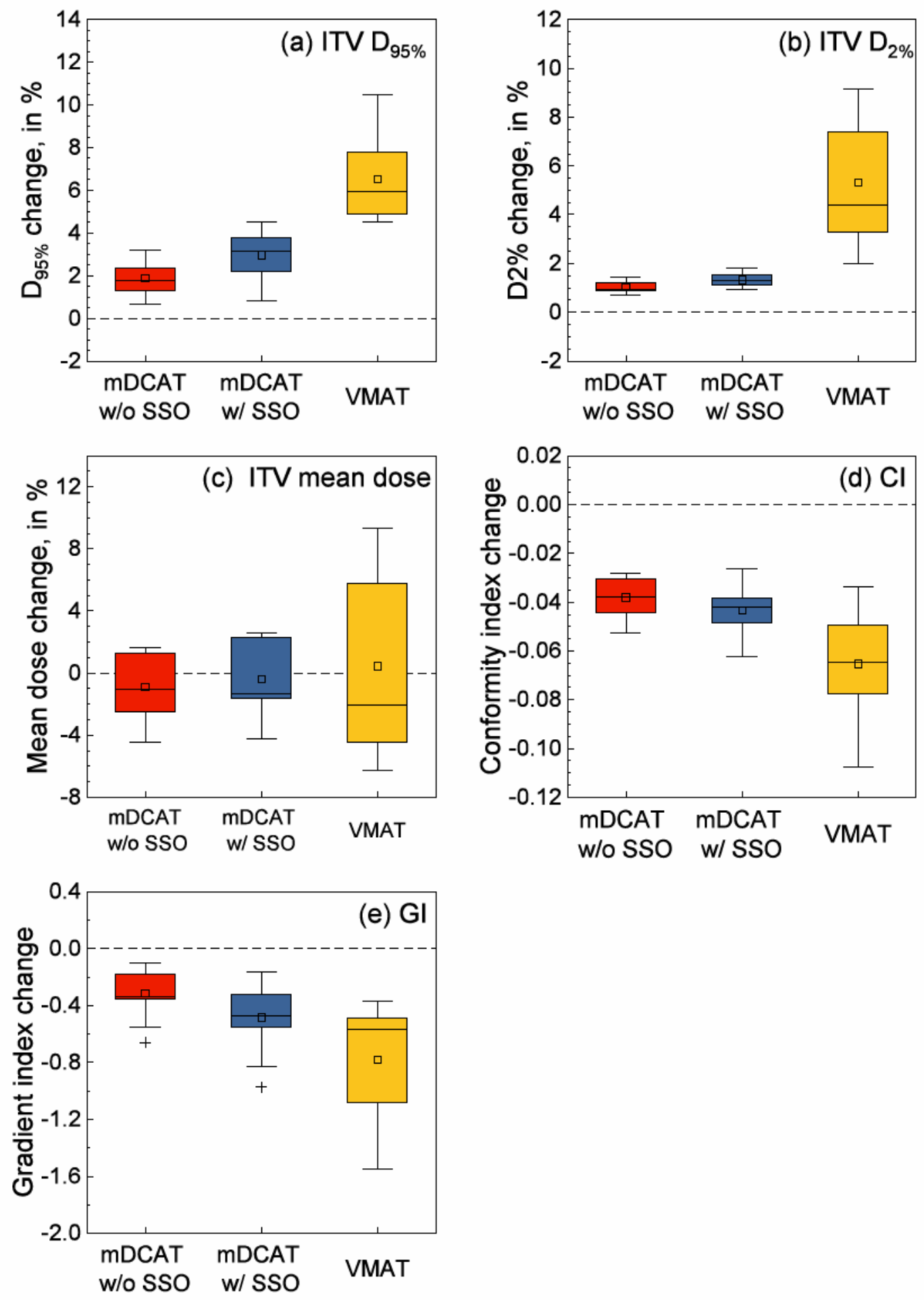

Figure 6.5: Same as in Figure 6.4, but for the twelve lung SBRT patients included in this study. 

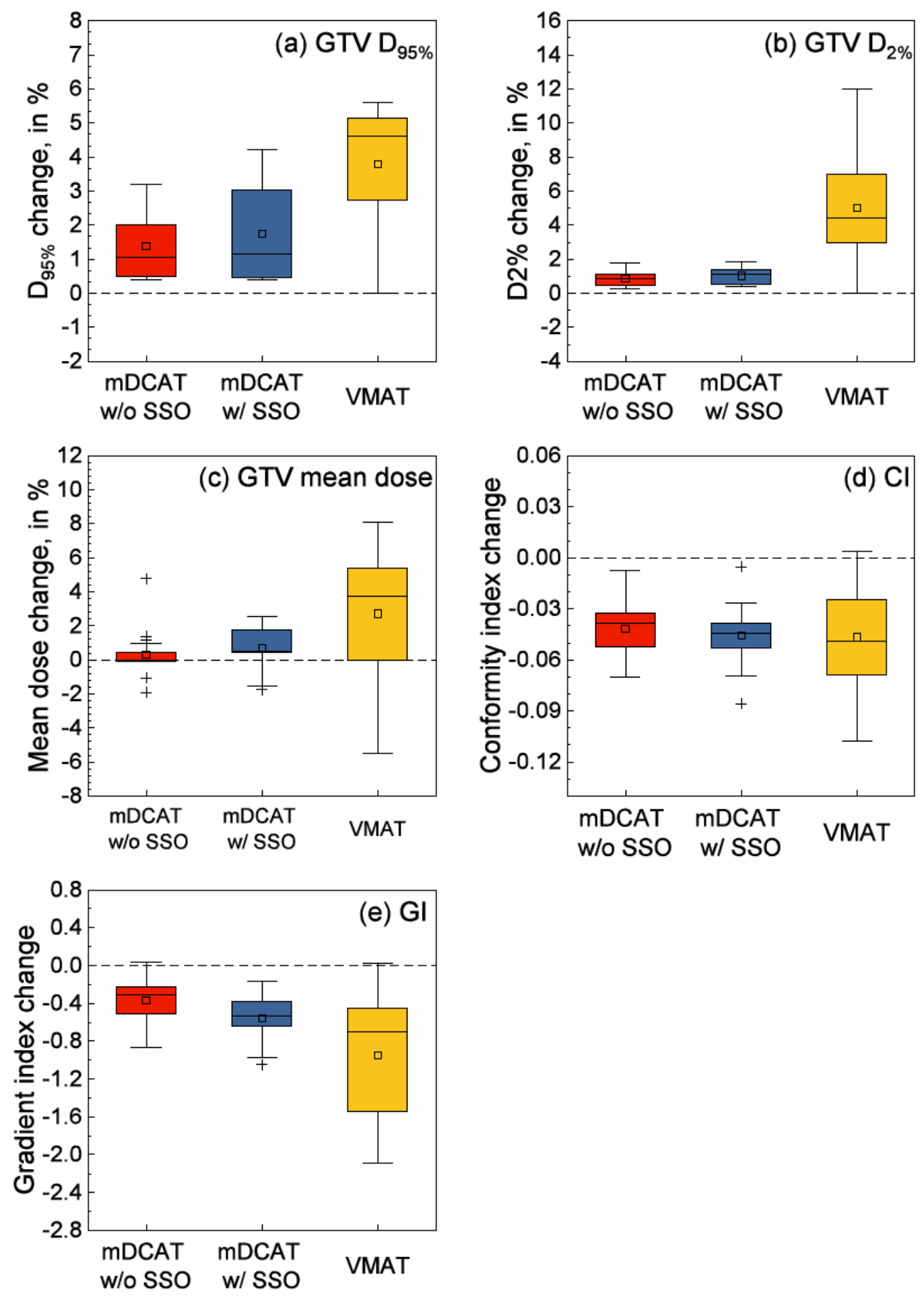

Figure 6.6: Same as in Figure 6.4, but for the twelve brain patients included in this study. 


\subsection{Dosimetric impact of typical MLC errors}

The results of the dosimetric impact of modelling typical systematic MLC errors are shown in Table 6.2 and Figures 6.7, 6.8 and 6.9. With the typical systematic MLC errors, the changes in the plan quality metrics were substantially lower compared to the changes in the metrics for the extreme systematic MLC errors. For the typical random errors (not shown in the table or boxplots), the changes in the plan quality metrics were comparable with the typical systematic errors in terms of magnitude and overall behaviour.

In Figures 6.7, 6.8 and 6.9 for the three disease sites, the changes in quality metrics for mDCAT had small spread (25\%-75\%) and extremes (whiskers and outliers) compared with VMAT which had larger spread. Even for the small perturbations shown in the plots, mDCAT was found to be more robust compared to VMAT, with average values of the plan quality metrics for mDCAT closer to the values of the base plan, and with less spread. The dose errors were still amplified for the lung SBRT and brain targets compared to those for the spine cases. However, all the changes were clinically insignificant for both VMAT and mDCAT. 
Table 6.2: Same as in Table 6.1, but for typical systematic MLC errors.

\begin{tabular}{|c|c|c|c|c|}
\hline \multirow{2}{*}{ Metric } & \multirow{2}{*}{ Technique } & \multicolumn{3}{|c|}{ Change in metric } \\
\hline & & Spine & Lung SBRT & Brain \\
\hline $\begin{array}{l}D_{95 \%} \\
\text { relative to } R x \text {, } \\
\text { in } \%\end{array}$ & $\begin{array}{l}\text { mDCAT w/o SSO } \\
\text { mDCAT w/SSO } \\
\text { VMAT }\end{array}$ & $\begin{array}{c}-0.00 \pm 0.02 \\
0.01 \pm 0.02 \\
-0.04 \pm 0.14\end{array}$ & $\begin{array}{l}-0.02 \pm 0.08 \\
-0.03 \pm 0.15 \\
-0.17 \pm 0.31\end{array}$ & $\begin{array}{l}-0.06 \pm 0.16 \\
-0.04 \pm 0.16 \\
-0.42 \pm 0.87\end{array}$ \\
\hline $\begin{array}{l}D_{2 \%} \\
\text { relative to } \mathrm{Rx} \text {, } \\
\text { in } \%\end{array}$ & $\begin{array}{l}\text { mDCAT w/o SSO } \\
\text { mDCAT w/ SSO } \\
\text { VMAT }\end{array}$ & $\begin{array}{l}0.00 \pm 0.02 \\
0.00 \pm 0.02 \\
0.16 \pm 0.41\end{array}$ & $\begin{array}{c}-0.03 \pm 0.02 \\
-0.01 \pm 0.07 \\
0.13 \pm 0.50\end{array}$ & $\begin{array}{l}-0.15 \pm 0.41 \\
-0.10 \pm 0.37 \\
-0.56 \pm 1.05\end{array}$ \\
\hline $\begin{array}{l}\text { Mean dose } \\
\text { relative to } \mathrm{Rx} \text {, } \\
\text { in } \%\end{array}$ & $\begin{array}{l}\text { mDCAT w/o SSO } \\
\text { mDCAT w/SSO } \\
\text { VMAT }\end{array}$ & $\begin{array}{l}0.00 \pm 0.01 \\
0.00 \pm 0.02 \\
0.01 \pm 0.07\end{array}$ & $\begin{array}{l}-0.03 \pm 0.05 \\
-0.07 \pm 0.06 \\
-0.14 \pm 0.15\end{array}$ & $\begin{array}{l}-0.38 \pm 1.34 \\
-0.38 \pm 1.61 \\
-0.15 \pm 3.81\end{array}$ \\
\hline $\mathrm{CI}$ & $\begin{array}{l}\text { mDCAT w/o SSO } \\
\text { mDCAT w/ SSO } \\
\text { VMAT }\end{array}$ & $\begin{array}{l}0.000 \pm 0.001 \\
0.000 \pm 0.002 \\
0.002 \pm 0.009\end{array}$ & $\begin{array}{c}0.001 \pm 0.001 \\
0.001 \pm 0.015 \\
-0.001 \pm 0.001\end{array}$ & $\begin{array}{l}0.013 \pm 0.011 \\
0.001 \pm 0.017 \\
0.041 \pm 0.063\end{array}$ \\
\hline HI & $\begin{array}{l}\text { mDCAT w/o SSO } \\
\text { mDCAT w/ SSO } \\
\text { VMAT }\end{array}$ & $\begin{array}{l}0.0001 \pm 0.0006 \\
0.0001 \pm 0.0003 \\
0.0016 \pm 0.0028\end{array}$ & see footnote & see footnote \\
\hline GI & $\begin{array}{l}\text { mDCAT w/o SSO } \\
\text { mDCAT w/ SSO } \\
\text { VMAT }\end{array}$ & $\begin{array}{l}0.01 \pm 0.03 \\
0.00 \pm 0.01 \\
-0.06 \pm 0.25\end{array}$ & $\begin{array}{l}0.01 \pm 0.02 \\
0.02 \pm 0.02 \\
0.02 \pm 0.03\end{array}$ & $\begin{array}{l}0.00 \pm 0.01 \\
0.04 \pm 0.15 \\
0.04 \pm 0.42\end{array}$ \\
\hline
\end{tabular}

HI not evaluated for stereotactic treatments. See Section 2.7 for discussion. 

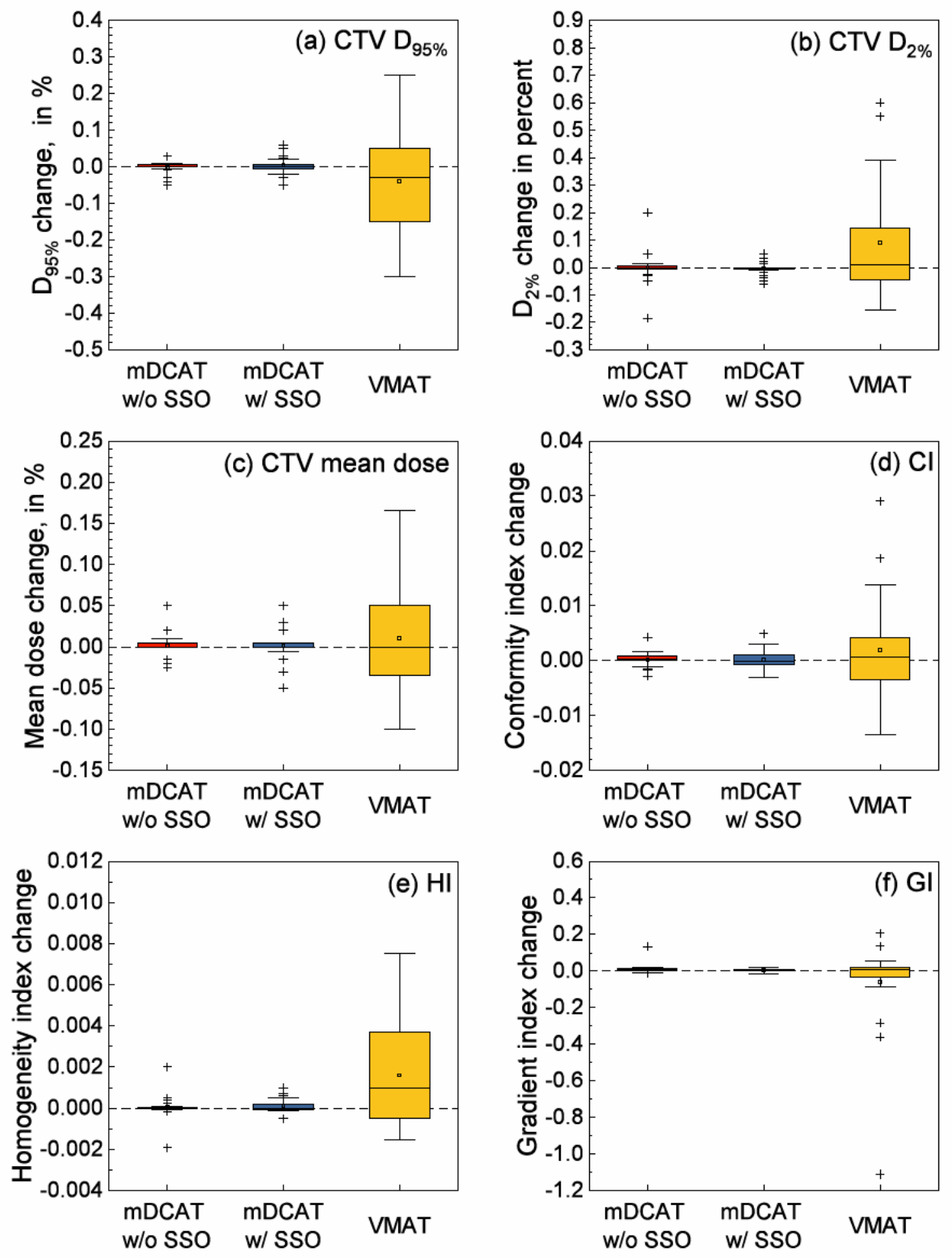

Figure 6.7: Boxplots of the changes in the values of plan quality metrics for the twenty five palliative patients included in this study after introducing typical systematic MLC errors from the QA data of the Agility collimation system at TOHCC. 

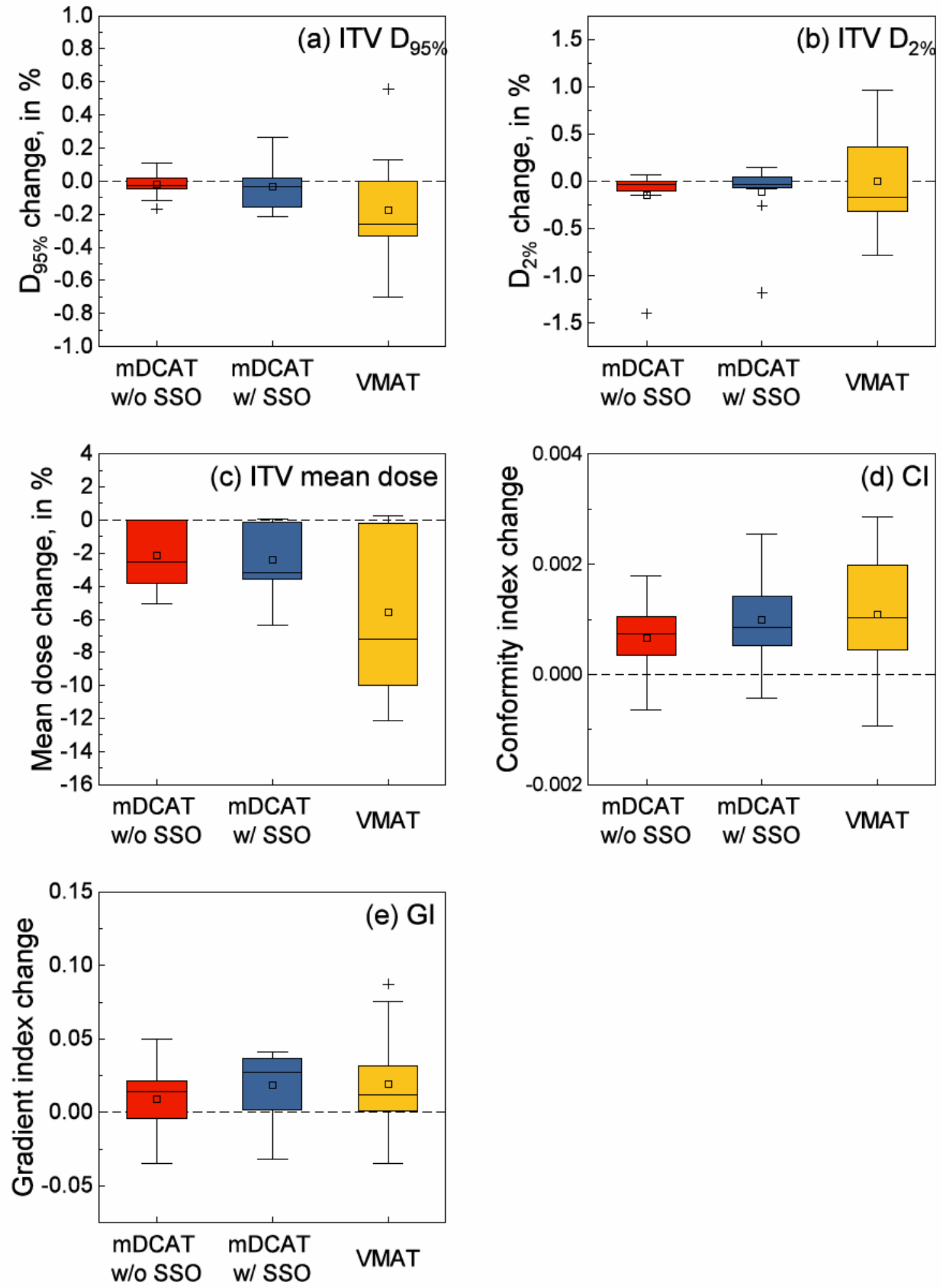

Figure 6.8: Same as in Figure 6.7, but for the twelve lung SBRT cases included in this study. 

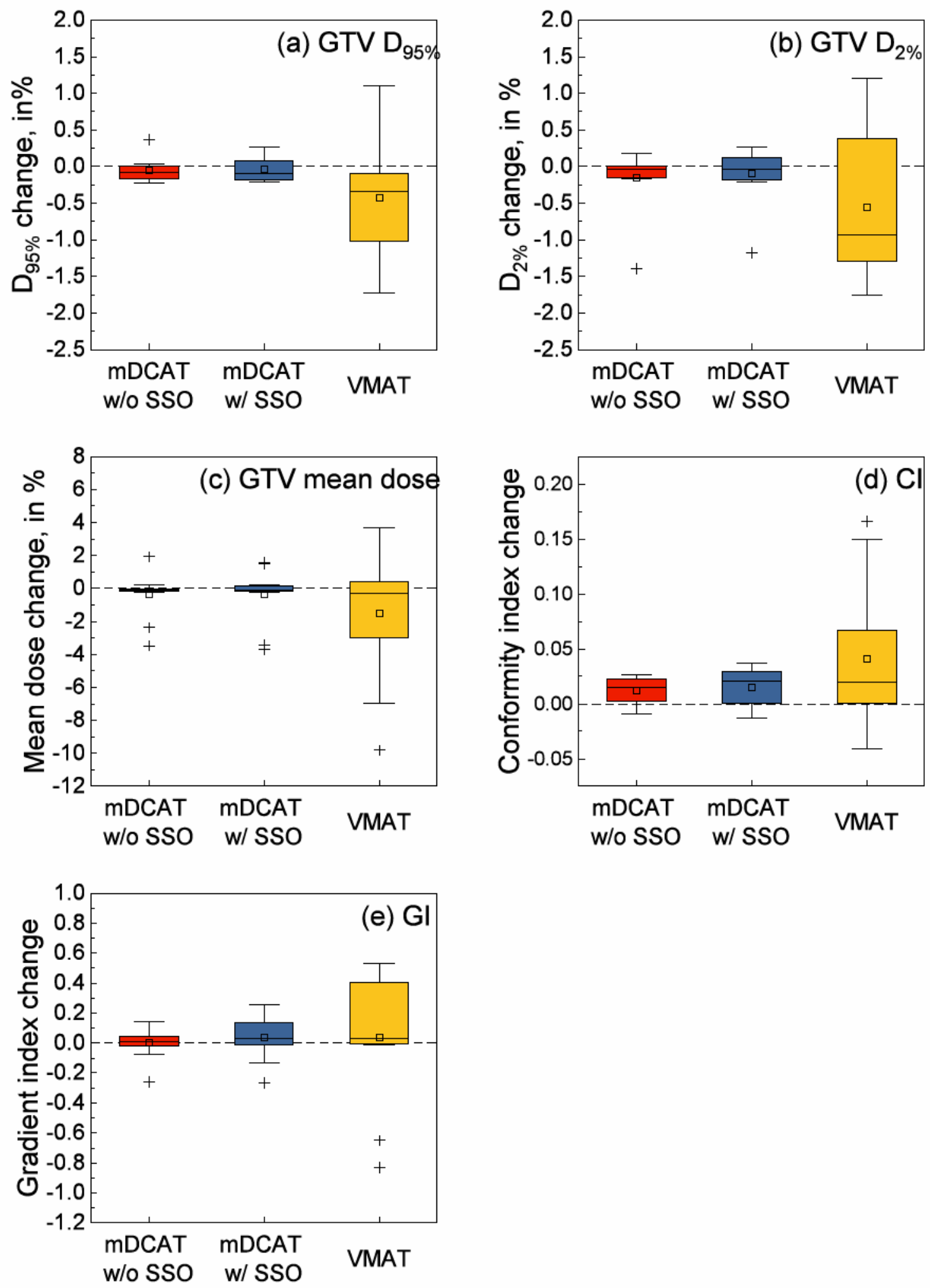

Figure 6.9: Same as in Figure 6.7, but for the twelve brain cases included in this study. 


\subsection{Summary}

Machine uncertainties are unavoidable during RT. The positional errors of the MLC leaves is one source of machine uncertainties, and these errors are relevant because of the extensive use of the MLC leaves during dynamic treatments (e.g., VMAT and mDCAT). The results from the clinical QA data of the Elekta Agility MLC systems at TOHCC, as well as the results from the experimental delivery of mDCAT and VMAT plans followed by analysis of the log files for these deliveries, show a maximum $\pm 1 \mathrm{~mm}$ range of the MLC leaf positional errors. The effect of these typical MLC errors on the plan quality of VMAT and mDCAT plans is small, regardless of the target size. mDCAT was shown to be more robust (with SSO being slightly worse) compared to VMAT, even at these small magnitude of perturbations to MLC positions. For extreme cases in which the MLC leaves are systematically off from their true positions by the tolerance value (i.e., $2 \mathrm{~mm}$ ), mDCAT substantially outperforms VMAT. The addition of SSO slightly worsens the robustness of mDCAT compared to that without SSO due to the added modulation. However, mDCAT with SSO is still more robust compared to VMAT against such extreme errors. 


\section{Chapter 7}

\section{Robustness against patient uncertainties}

Patient-related uncertainties are typically encountered during RT. ${ }^{100,101}$ These uncertainties include inter- and intra-fraction patient positioning variability, anatomical changes (weight gain or loss, edema, tumour shrinkage, etc), and physiological movement during beam-on time (breathing, swallowing, heart beating, bladder filling, rectal filling, etc). In this chapter, patient breathing motion was modelled without and with setup errors, and its dosimetric impact on mDCAT and VMAT plans was assessed to evaluate the robustness of both techniques against such uncertainties. Only the twelve lung SBRT patients were studied in this chapter because breathing motion is most relevant for small lung tumours.

The tables and graphs in this chapter summarize the results quantitatively. This is followed by a qualitative description of those results and their clinical relevance in the body of the text.

\subsection{Modelling tumour motion due to breathing}

As a patient breathes during treatment, the tumor in the lung can move and/or deform. ${ }^{100,101}$ The effect of breathing motion has been characterized as "blurring" of the spatial dose distribution. ${ }^{102}$ Dose blurring can cause under-dosage to the target and over-dosage to surrounding OARs if the motion is not properly accounted for during 
treatment planning (e.g., by using sufficient internal margin and creating an ITV see Figure 1.4), and/or during treatment delivery (e.g., by using techniques such as breath-hold, gating or tumor tracking). ${ }^{76}$

The pattern of tumour motion due to breathing is commonly represented as a sinusoidal, ${ }^{103}$ and it is the model used in this study. The introduced tumour motion was in the superior-inferior direction only because breathing causes lung tumours to move mostly in the superior and inferior directions. ${ }^{100,101}$ This was also verified for the twelve patients analyzed in this study where the GTV-to-ITV margin was found to be of the order of $3 \mathrm{~mm}$ in the superior-inferior direction and less than $1 \mathrm{~mm}$ in the other two orthogonal directions. The tumour motion during the breathing cycle was divided into ten phases equally-spaced in time (Figure 7.1). The location of the centre of the tumour in each phase was calculated from the value of the sinusoidal function at the mid-point of each phase. Two amplitudes for tumour motion were used in this study to represent extreme and typical motion. The extreme amplitude represents the situation in which patient breathing during the treatment delivery goes beyond the ITV range observed during the CT simulation stage. During CT simulation of lung patients at TOHCC, if the extent of tumor motion from the simulation scan is found to be greater than $1 \mathrm{~cm}$, the patient is re-scanned with an abdominal compression device to reduce the breathing amplitude (see Section 1.3). For this reason, the value of the extreme amplitude of tumour motion was taken as $1 \mathrm{~cm}$ in this study. On the other hand, the typical amplitude represents the situation in which the tumour motion range covered by the ITV during the CT simulation is replicated at the time of treatment. The typical breathing amplitude used in this study was extracted individually for each of the twelve lung SBRT patients using the distance in the superior-inferior direction from the edge of the GTV in the baseline phase to the edge of the ITV (i.e., the internal margin). 


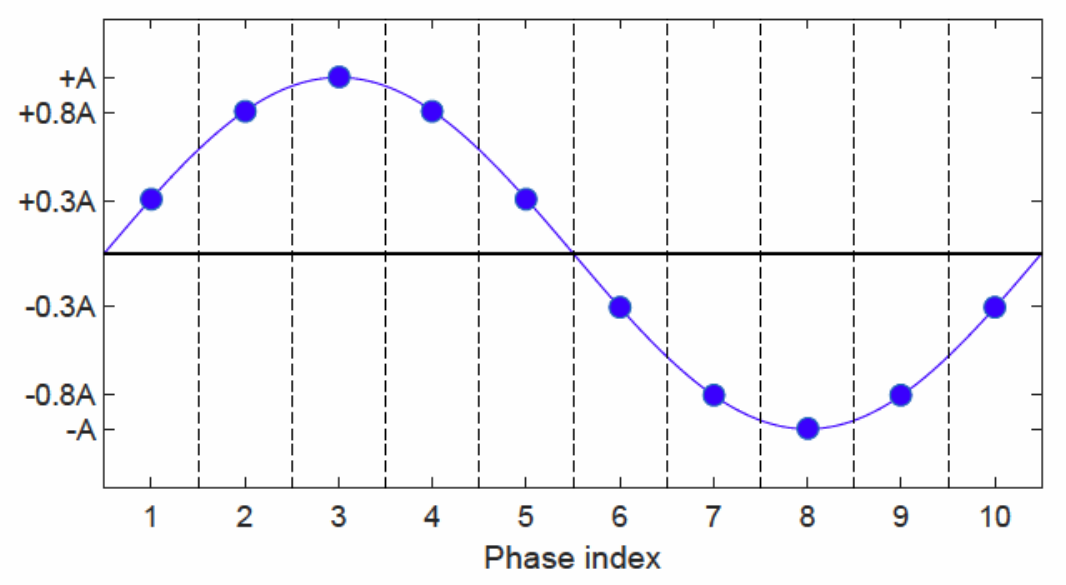

Figure 7.1: Sinusoidal pattern of tumour motion in the superior-inferior direction as used in this study. The motion cycle was divided into ten phases, in which the tumour centre was assumed to be in the location corresponding to the middle of each phase. A is the amplitude of the maximum extent of tumour motion.

The breathing periods used for this study were 3 and 6 seconds, which represent typical shallow and deep breathing, respectively.

Modelling tumour motion in the literature has been carried out using approaches with different levels of sophistication, depending on the end point of the investigations. ${ }^{23,29,104}$ These approaches are classified in this study into six categories in increasing level of sophistication, and two of these approaches were used in this study. (1) The simplest approach is to offset the dose distribution multiple times by the different magnitudes of tumour motion in the various phases (see Figure 7.1) and then sum the results to produce the final dose distribution. ${ }^{105,106}$ This simple convolution method does not take into account the patient anatomy changes since the shape of the offset dose distributions is identical to the original distribution. (2) The isocentre of the treatment plan is moved multiple times by the different magnitudes of tumour motion in the various phases. A separate dose calculation is performed on the average scan (see Section 1.3) at each isocentre location, and the results are summed to produce the final distribution. Unlike \#1, this approach accounts for anatomy changes relative to the 
treatment plan. However, it assumes that the breathing frequency is much faster than the delivery such that all segments in the delivered plan (on average) see all phases of the breathing cycle. Also, since the same average CT scan is used for all calculations, this approach does not account for the variation in patient anatomy at each phase, including tumour deformation and its location relative to the rest of the patient anatomy. Finally, the discretization of the tumour motion into ten bins is a secondary approximation (versus its continuous motion in reality). (3) Same as \#2 where the average CT scan is used for the dose calculations at all isocentre locations, but the "interplay" between the breathing cycle and the treatment delivery is taken into account whereby different isocentre moves (which correspond to different phases in the breathing cycle) are associated with only the specific parts of the treatment delivery that fall in that bin of the breathing cycle. Such calculations are typically repeated multiple times with different starting points of the breathing cycle relative to the start of the treatment plan delivery. The result is a "band of solutions" that represents the variability in the dose distribution due to the "interplay" between the plan delivery and the tumour motion. (4) Same as \#2 except that the dose distribution at each isocentre location is calculated on the CT scan that corresponds to that particular breathing phase, rather than on the average scan. This faithfully represents the variation in patient anatomy at each phase, including the tumour location relative to the rest of the patient anatomy. The resulting dose distributions cannot be simply added up because they are calculated on different CT scans. Rigid or deformable image registration and dose mapping methods are used to produce the final dose distribution. ${ }^{107-111}$ (5) A combination of \#3 and \#4 whereby the "interplay effect" (as in \#3) is modelled but on different CT scans representing the different phases (as in \#4). Rigid or deformable image registration and dose mapping methods are required to produce the final dose calculation. (6) The most sophisticated approach is through continuous (rather than discretized) modelling 
of the tumour motion using random sampling and full 4D Monte Carlo simulations with on-the-fly deformable dose mapping methods. ${ }^{107-112}$

In this study, both of approaches \#2 and \#3 described above were used. These approaches have been shown to sufficiently scope most of the dosimetric impact of breathing motion. ${ }^{107,113,114}$ The details of the implementation are described in Section 7.2 for approach \#2, and in Section 7.4 for approach \#3. The value in implementing approach \#2 is that it represents an asymptotic case of the more accurate approach \#3. The more sophisticated approaches beyond approach \#3 involve image registration and dose mapping, and are beyond the scope of this study.

For the reasons explained in the last two paragraphs of Section 2.4, the finite size Pencil Beam dose calculation algorithm was used for the robustness calculations for the plans with the breathing motion modelled, and for their corresponding base plans without the motion, and it has been shown previously (see Tables 2.1 and 2.2) that the choice of this dose calculation algorithm does not negatively impact the accuracy of robustness calculations for small perturbations. The voxel size used for all calculations was $2 \mathrm{~mm}^{3}$, which is suitable for small lung SBRT targets. The dosimetric impact of breathing motion was assessed by comparing the dose distributions with versus without the motion modelling, and evaluating the change in the plan quality metrics (plan quality metrics were defined in Section 2.7). The GTV was used for the evaluation, rather than the ITV because the isocentre movements mirror the ITV to model the impact of motion of the GTV target. 


\subsection{Modelling tumour motion using isocentre-moves only}

In this modelling approach, the time frame of the breathing cycle is assumed to be much shorter than the time frame of the delivery details of the plan segments, therefore the length of the breathing period is not explicitly needed, and all parts of the plan delivery equally see all breathing phases. This was modelled by dividing the treatment plan into ten sub-plans, corresponding to the ten breathing phases. Each sub-plan had the exact beam segments as the original plan without motion, but with the isocentre of the sub-plan shifted in the superior-inferior direction by the magnitude of the tumour motion in the respective breathing phase. Each sub-plan has one-tenth of the total MUs of the original plan. All sub-plans were calculated on the average CT and added to produce the final dose distribution that accounts for tumour motion.

\subsection{Dosimetric impact: isocentre-moves only}

The results of the dosimetric impact of modelling tumour motion with only isocentre moves are shown in Table 7.1 and Figure 7.2. Table 7.1 shows the average changes in the plan quality metrics and their standard deviations, and Figure 7.2 show the corresponding boxplots for the changes in the plan quality metrics. Good robustness against a modelled error is manifested in the metrics of plan quality in two ways: (1) the mean values of the metrics remain close to their values for the original plans without the modelled error (represented by the dashed lines in the panels), and, (2) the spread around the mean values of the change in the metrics are small (represented by a small size of the boxes in the boxplots, and by shorter whiskers and less outliers).

For the extreme tumour motion amplitude, Table 7.1 and Figure 7.2 show that 
mDCAT is less robust than VMAT in terms of target coverage, with a larger change in the GTV D ${ }_{95 \%}$. This can be explained as follows. The typical GTV-to-ITV margin used for the lung SBRT cases in this study was $3 \mathrm{~mm}$, and the "setup margin" (see Figure 1.4) added to create the PTV for the lung SBRT cases presented in this study was $5 \mathrm{~mm}$, which resulted to a total GTV-to-PTV margin of $8 \mathrm{~mm}$. A 1-cm motion placed the 2 mm most superior and most inferior parts of the GTV outside the beam edges at the breathing peaks (phases 3 and 8 of the tumour motion cycle shown in Figure 7.1) during mDCAT delivery, which leads to the results of $\mathrm{D}_{95 \%}$ shown in Table 7.1 and Figure 7.2. Adding SSO to mDCAT provides some improvement in robustness in $\mathrm{D}_{95 \%}$. In VMAT, the beam segments have more complex shapes, which leads to higher tendencies for extreme individual deviations (the large box plots and the outliers $(+)$ in Figure 7.2 for VMAT). For the GTV $D_{2 \%}$, the changes for mDCAT was lower compared to VMAT. The changes in the GTV mean dose, CI, GI and the healthy lungs $\mathrm{V}_{20 G y}$ for the VMAT and mDCAT were comparable.

For the typical amplitude tumour motion, the magnitude of changes in the plan quality metrics was substantially reduced compared to that with the extreme tumour motion, as shown in Table 7.1. The GTV coverage for VMAT and mDCAT deviated from the original coverage by less than one percent, on average. This leads to the conclusion that mDCAT and VMAT are equally robust against typical motion uncertainties, and within this small range of change, VMAT has the largest spread due to the complexity of the shapes of the beam segments in VMAT. 
Table 7.1: Average changes in the plan quality metrics and their standard deviations after introducing extreme and typical tumour motion using only isocentre-moves for the mDCAT and VMAT plans for the twelve lung SBRT patients included in this study. The extreme amplitude used in this study is $1 \mathrm{~cm}$, and the typical amplitude is the individual GTV-to-ITV margin for each patient (about $3 \mathrm{~mm}$ ). The metrics are evaluated for the GTV.

\begin{tabular}{|c|c|c|c|}
\hline \multirow[b]{2}{*}{ Metric } & \multirow[b]{2}{*}{ Technique } & \multicolumn{2}{|c|}{ Change in metric } \\
\hline & & $\begin{array}{c}\text { Extreme } \\
\text { amplitude }\end{array}$ & $\begin{array}{c}\text { Typical } \\
\text { amplitude }\end{array}$ \\
\hline $\begin{array}{l}D_{95 \%} \\
\text { relative to } \mathrm{Rx} \text {, } \\
\text { in } \%\end{array}$ & $\begin{array}{l}\text { mDCAT w/o SSO } \\
\text { mDCAT w/SSO } \\
\text { VMAT }\end{array}$ & $\begin{array}{l}-3.0 \pm 1.9 \\
-2.0 \pm 0.4 \\
-0.3 \pm 0.9\end{array}$ & $\begin{array}{c}-0.2 \pm 0.4 \\
-0.1 \pm 0.1 \\
0.2 \pm 0.6\end{array}$ \\
\hline $\begin{array}{l}D_{2 \%} \\
\text { relative to } \mathrm{Rx} \text {, } \\
\text { in } \%\end{array}$ & $\begin{array}{l}\text { mDCAT w/o SSO } \\
\text { mDCAT w/SSO } \\
\text { VMAT }\end{array}$ & $\begin{array}{l}-0.9 \pm 0.3 \\
-1.1 \pm 0.2 \\
-0.8 \pm 4.7\end{array}$ & $\begin{array}{l}-0.1 \pm 0.1 \\
-0.2 \pm 0.1 \\
-0.8 \pm 0.9\end{array}$ \\
\hline $\begin{array}{l}\text { Mean dose } \\
\text { relative to } \mathrm{Rx} \text {, } \\
\text { in } \%\end{array}$ & $\begin{array}{l}\text { mDCAT w/o SSO } \\
\text { mDCAT w/SSO } \\
\text { VMAT }\end{array}$ & $\begin{array}{l}-1.6 \pm 0.6 \\
-1.5 \pm 0.7 \\
-1.1 \pm 2.3\end{array}$ & $\begin{array}{l}-0.2 \pm 0.1 \\
-0.2 \pm 0.1 \\
-0.3 \pm 0.5\end{array}$ \\
\hline CI & $\begin{array}{l}\text { mDCAT w/o SSO } \\
\text { mDCAT w/SSO } \\
\text { VMAT }\end{array}$ & $\begin{array}{l}0.05 \pm 0.02 \\
0.00 \pm 0.01 \\
0.06 \pm 0.03\end{array}$ & $\begin{array}{l}0.00 \pm 0.01 \\
0.05 \pm 0.02 \\
0.00 \pm 0.01\end{array}$ \\
\hline GI & $\begin{array}{l}\text { mDCAT w/o SSO } \\
\text { mDCAT w/ SSO } \\
\text { VMAT }\end{array}$ & $\begin{array}{l}1.7 \pm 0.8 \\
2.0 \pm 1.0 \\
1.6 \pm 0.6\end{array}$ & $\begin{array}{l}0.1 \pm 0.1 \\
0.1 \pm 0.1 \\
0.1 \pm 0.1\end{array}$ \\
\hline $\begin{array}{l}\text { Healthy lungs } \\
\mathrm{V}_{20 G y}\end{array}$ & $\begin{array}{l}\text { mDCAT w/o SSO } \\
\text { mDCAT w/ SSO } \\
\text { VMAT }\end{array}$ & $\begin{array}{l}-0.24 \pm 0.04 \\
-0.23 \pm 0.04 \\
-0.20 \pm 0.01\end{array}$ & $\begin{array}{l}-0.02 \pm 0.02 \\
-0.02 \pm 0.02 \\
-0.02 \pm 0.01\end{array}$ \\
\hline
\end{tabular}



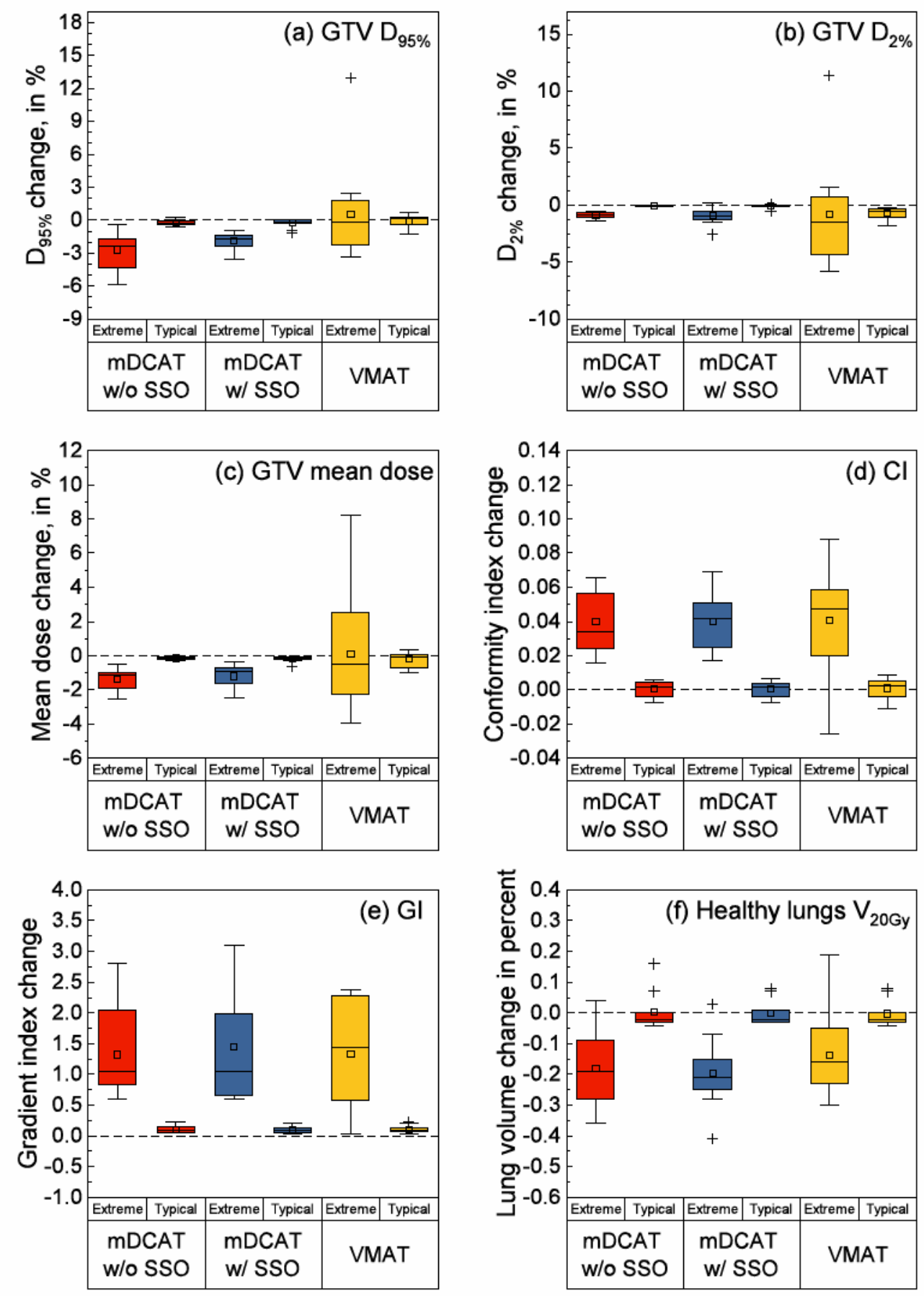

Figure 7.2: Boxplots of the changes in the values of plan quality metrics for the twelve lung SBRT patients included in this study after introducing extreme and typical tumour motion using isocentre-moves only. The extreme amplitude used in this study is $1 \mathrm{~cm}$, and the typical amplitude is the individual GTV-to-ITV margin for each patient (about $3 \mathrm{~mm}$ ). The metrics are evaluated for the GTV. 


\subsection{Modelling interplay effect}

This modelling approach is substantially more involved than that described in Section 7.2. Modelling the interplay between the breathing cycle and the treatment delivery required addressing four steps. (1) Knowledge of the characteristics of the LINAC components versus time during the treatment. (2) Breaking down the treatment plan into different sub-plans, each of which contains the collection of the parts of the delivery that occurred during a given breathing phase. (3) Efficient dose calculations of the ten sub-plans, and summing them to produce the final dose distribution. (4) Changing the starting point of the breathing cycle versus the start of the treatment and repeating the previous steps to evaluate the robustness against the interplay effect. Each of those four steps is discussed in turn below.

Step 1: Treatment plan details versus time: As discussed in Section 2.2.6, each treatment plan consists of a series of segments, and each segment has a control point that defines the beginning of the segment and another control point that defines the end fo that segment. Each control point defines the status of different machine components at that point in the delivery, which are: the position of the gantry (single value), the position of each of the two solid jaws (2 jaws x 1 position value each), the position of each MLC leaf in the two MLC leaf banks (2 banks x 80 MLC leaf position values), the position of each of the two carriages that carry the two MLC leaf banks (2 carriages x 1 position value each), and the number of MUs that should be delivered in the segment that ends with that control point. The control point at the end of a segment is the control point at the beginning of the following segment. The treatment plan files at the back end of the TPS contain the information about each control point, an example of which is shown in Figure 7.3. The LINAC system has limitations on the 


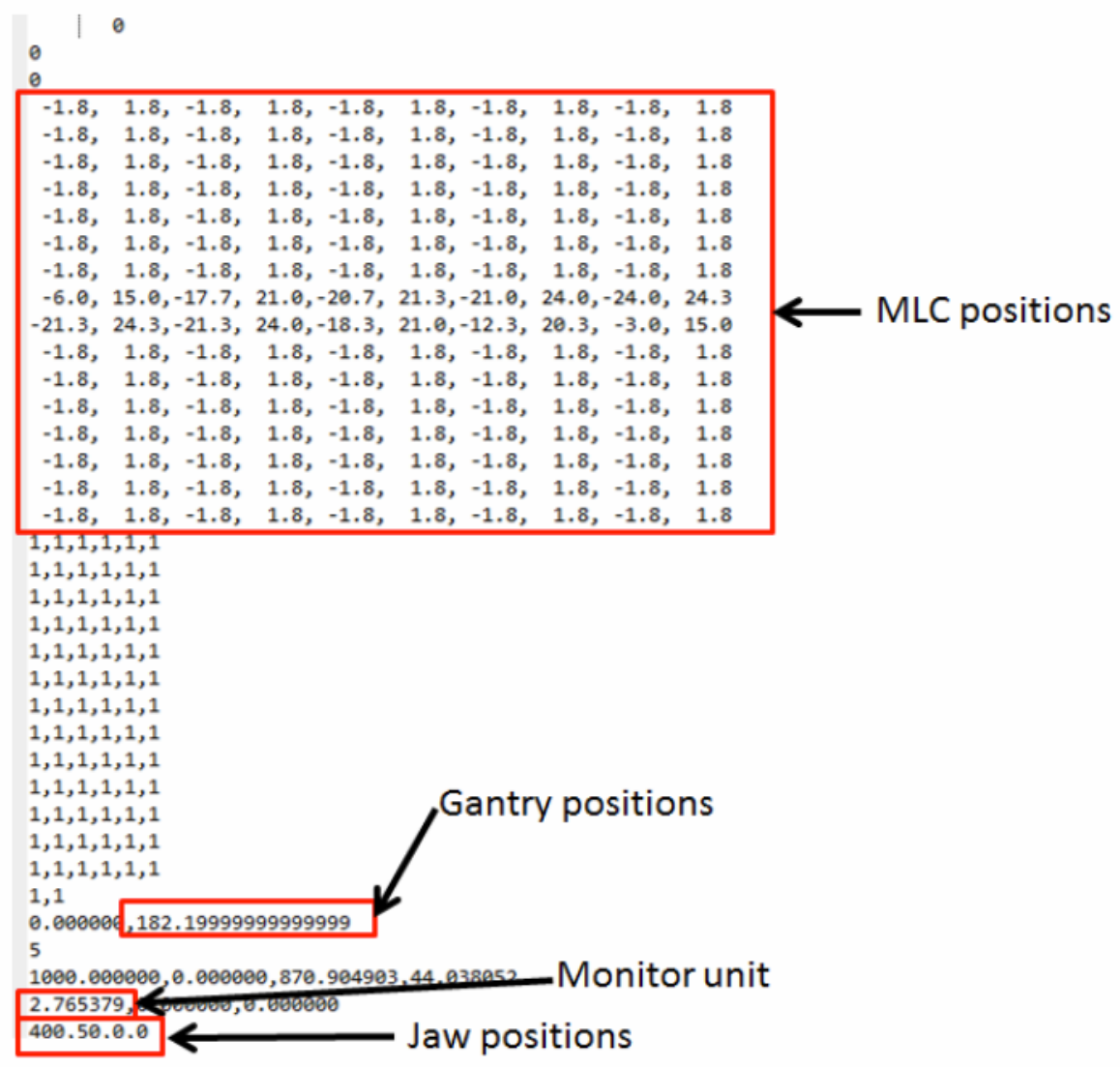

Figure 7.3: A screenshot of the treatment plan file at the back end of the Monaco TPS, in which different LINAC component positions per control point are defined. Labelled are the MLC leaf positions, gantry position, jaw positions and the number of MUs that should be delivered in the segment that ends with the displayed control point.

dynamic parameters of its physical components. For the Elekta system used in this study, the limitations on the dynamic parameters relevant for this part of the study are: maximum dose rate of $10 \mathrm{MU}$ per second, maximum gantry speed of 6 degrees per second, maximum jaw speed of $90 \mathrm{~mm}$ per second, maximum speed for individual MLC leaves of $35 \mathrm{~mm}$ per second, and maximum speed of MLC carriage of $60 \mathrm{~mm}$ per second - see Figure 2.2 for limitations on other dynamic parameters. During the actual delivery of the treatment, the Linac Control System (LCS) uses the information of the control points from the TPS, in combination with the maximum physical limitations on the dynamic parameters to determine for each segment a single value for each component 
during the delivery of that segment namely a value for dose rate in that segment, a gantry speed, speeds of individual MLC leaves, and speeds of each of the two jaws and MLC carriages. In this study, a code was developed in the Python ${ }^{99}$ scripting language to model the algorithm of the LCS as follows. The goal is to achieve the most time-efficient delivery for each segment. From the control points at the start and end of each segment, the amount of travel needed by each component (including each individual MLC leaf), plus the MUs for that segment are determined. Using this knowledge and the maximum limits on each component, the minimum time required by each component for that segment is determined. The longest of these minimum times is considered the bottleneck, and is used as the delivery time for that segment. Using this delivery time and the known distances to be travelled in the segment, the actual speed of each component is determined during that segment. This process was repeated independently for all segments in the treatment plan. The end result is that the state of all relevant linac components continuously versus time becomes known for the entire duration of the treatment.

To validate the Python algorithm developed in this study, the treatment plans of mDCAT with SSO and VMAT for six patients from each disease site (spine, lung and brain) were experimentally delivered on the LINAC, and the log files of the deliveries (with 40 millisecond sampling) were extracted and analyzed. Shown in Figures 7.4 and 7.5 is the comparison between the simulated and the actual delivery for the dynamic components for each segment for mDCAT with SSO and for VMAT, respectively. Figure 7.6 shows the simulated versus measured delivery time for each segment. Overall, the simulation results are in excellent agreement with experimental measurements. The discrepancies are due to live adjustments that the LCS makes to compensate for live fluctuations in the performance of various components during the actual delivery. For 
mDCAT plans, the bottleneck (which determined the treatment time for each beam segment), was mainly the dose rate. The MLC leaves and the jaws mostly followed the shape of the target for mDCAT, which was reflected in the relatively slower speed of the MLC leaves and jaws, which were well below the maximum allowable speeds (see Figure $7.4 \mathrm{c}$ to $7.4 \mathrm{f}$ ). By contract for VMAT, the motion of the LINAC components were much more dynamic, with higher values for the component speeds. On average, the MLC leaf speeds and jaw speeds were, respectively, 2 and 5 times faster for VMAT relative to mDCAT. A few of the mDCAT and VMAT treatment plans were delivered on multiple LINACs (not shown), and the level of agreement between simulated data and experimental delivery was very comparable to that shown in Figures 7.4 and 7.5.

Step 2: Breaking down the treatment plan into time-resolved sub-plans: With knowledge of the treatment details versus time (from step 1) and knowledge of the breathing phases versus time, the treatment plan was divided into ten sub-plans, each of which contained only the specific parts of the treatment delivery that fell in the time period of a given bin in the breathing cycle. The fractional MUs and the location of the dynamic LINAC components at the start and end of each breathing phase were calculated using the Python algorithm developed in this study. Fictitious "move-only" segments had to be created in the sub-plans to move the linac components without delivering dose in the gaps between the parts of the delivery that did not fall in the breathing phase for that sub-plan. Once the sub-plans that correspond to each phase were prepared, the isocentre for each sub-plan was shifted in the superior-inferior direction by the magnitude of the tumour motion in the respective breathing phase. The process was done for both shallow and deep breathing, which have periods of 3 and 6 seconds, respectively. 

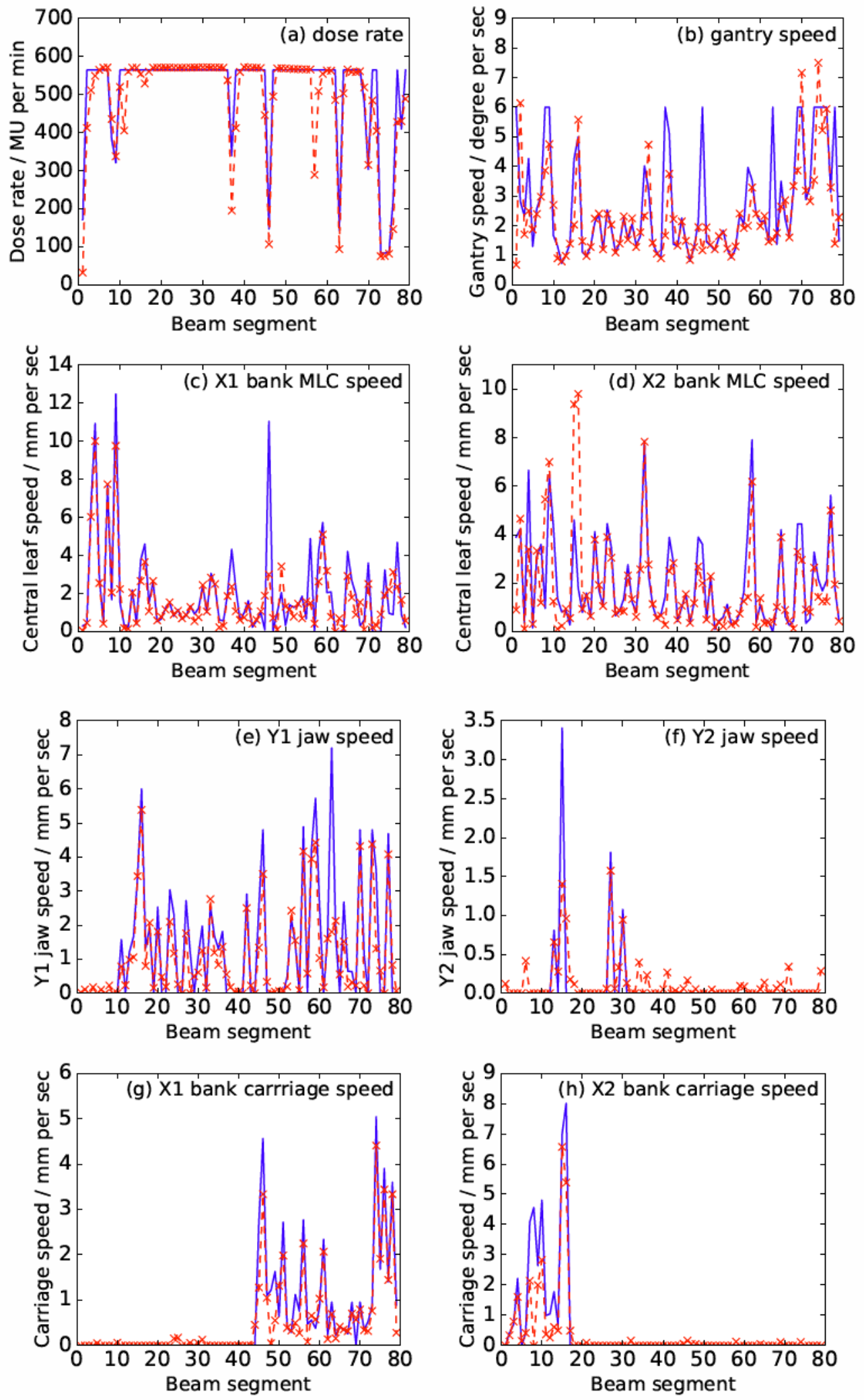

Figure 7.4: Dynamic parameters for each segment in an mDCAT with SSO plan for a typical lung SBRT case. Experimental data are in red $\mathrm{x}$ marks and simulated data are in solid blue lines. 

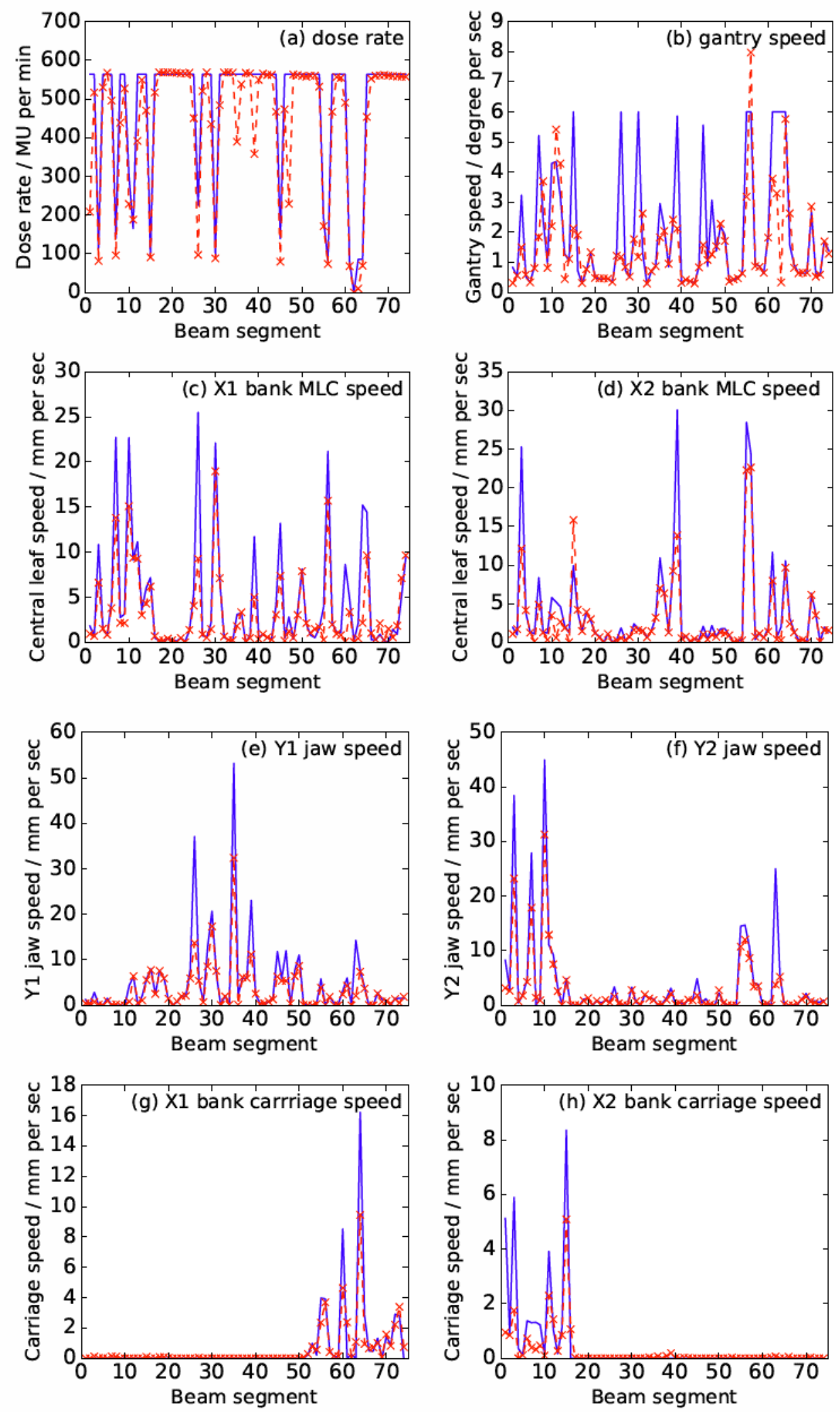

Figure 7.5: Same as in Figure 7.4, but for the corresponding VMAT plan for the same patient. Note the difference in the scale of the ordinate between Figures 7.4 and 7.5 for panels $\mathrm{c}, \mathrm{d}$, e and $\mathrm{f}$. 

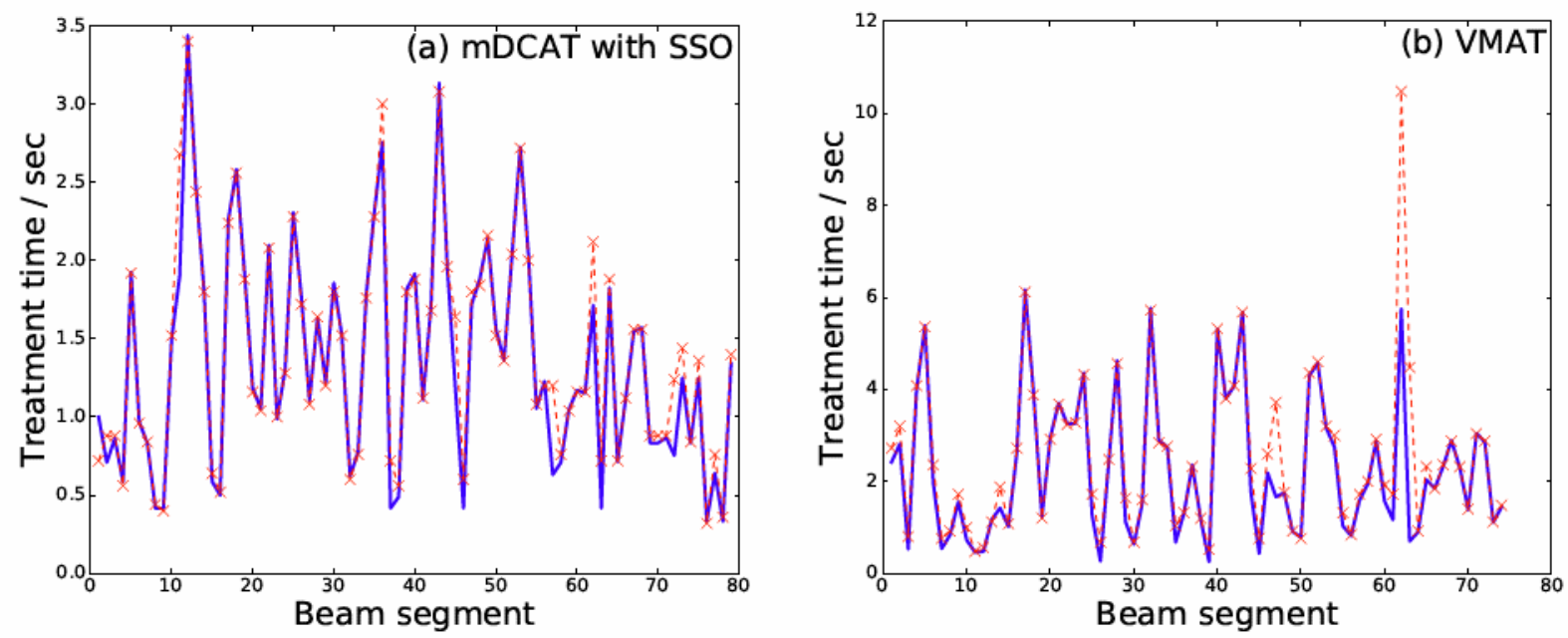

Figure 7.6: Same as in Figure 7.4, but for the treatment time of each segment. Note the difference in the scale of the two ordinates.

Step 3: Efficient dose calculations: The original plans and all sub-plans were calculated on the average CT scan. The sub-plans were added to produce the final dose distributions that account for tumour motion. As mentioned in Section 2.4, the finite size Pencil Beam algorithm was used for dose calculations that assess the impact of the small perturbations of breathing motion. A subtle issue in finite size Pencil Beam related to the discretization of source location in the dose calculations arose from creating the time-resolved sub-plans and was addressed as follows. In the finite size Pencil Beam implementation in the Monaco TPS, the radiation source is placed in the middle for each beam segment (unlike in the Monte Carlo implementation where the radiation source is modelled continuously using random sampling). During the manipulation of the size of the segments in Step 2 to create the time-resolved sub-plans, the Monaco TPS shifts the location of the source to the middle of the new segments during the dose calculations. To test this in isolation, time-resolved sub-plans were created, but the isocentre of all ten sub-plans was kept the same as the isocentre of the original plan. The dose was calculated and summed for the ten sub-plans and compared to 
the original plan. Without an isocentre moves in the sub-plans, the cumulative dose distribution should be identical to the original distribution, because finite size Pencil Beam is a deterministic calculation (rather than stochastic such as in Monte Carlo). However, due to the discretization of source location, differences up to a maximum of $0.7 \%$ of $\mathrm{Rx}$ were observed. To circumvent this artefact for the purpose of robustness assessment in this study, the sum of the ten sub-plans without isocentre moves was used as the base plans for comparison to the sum of the ten sub-plans with isocentre moves. This approach identically eliminated the artefact caused by source digitization. Additionally, Monte Carlo calculations were performed, and they confirmed the finite size Pencil Beam results for the impact of breathing motion to within the $0.5 \%$ statistical uncertainty of the Monte Carlo calculations.

Step 4: Changing the starting point of the breathing cycle: For the same beam segment delivery, the dosimetric impact of breathing motion varies depending on the phase(s) of the breathing cycle that occur during that segment, an extreme example of this is schematically illustrated in Figure 7.7. To model this interplay, the three steps above were repeated for five different starting phases for mDCAT and VMAT plans, and for both breathing periods of 3 and 6 seconds.

\subsection{Dosimetric impact: interplay effect}

The first set of results presented in this section are for only one particular starting phase of the breathing cycle (which represents the methods up to the end of step 3 in the four steps discussed above). After the general behavior is discussed, the results of the interplay effect with five different starts of the breathing cycle are presented. 

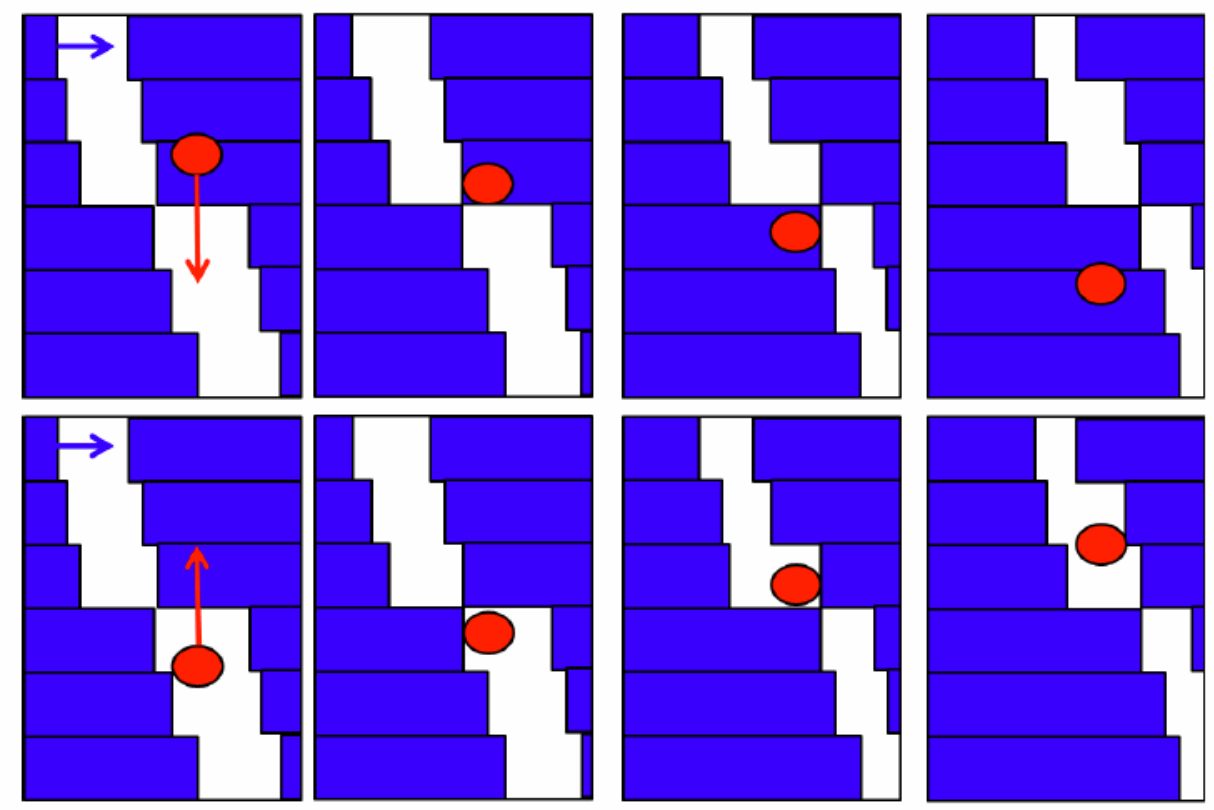

Figure 7.7: A schematic illustration to show how the phase in the breathing cycle can affect the dose received by the target for the same segment delivery. The red circle is the GTV, and it is moving in the superior-inferior direction during breathing (top-bottom of the page). The blue rectangles are MLC leaves performing a sweeping motion from left to right during the delivery of a segment. The white space is the open radiation field. The top and bottom rows are for two different breathing phases in the breathing cycle. The top row is for deep exhale, and the bottom row is for deep inhale. In this extreme example, the target is always outside of the open field in the top row, and always inside the open field in the bottom row, which leads to very different dose delivered to the target during that same segment.

\subsubsection{One start of the breathing cycle}

The results for the interplay case where the start of the breathing cycle coincides with the start of the treatment delivery for the twelve lung SBRT patients included in this study are shown in Table 7.2 (the two columns without setup error) and in Figure 7.8 for the breathing period of 6 seconds.

For the extreme amplitude of tumour motion without setup errors, Table 7.2 and Figure 7.8 show that the changes in the $\mathrm{D}_{95 \%}$ and in the mean dose to the GTV were higher for mDCAT compared with those for VMAT. The average changes in the GTV 
$\mathrm{D}_{2 \%}$ was lower for mDCAT compared with VMAT. The spread of the changes in plan quality metrics (height of the boxplots) was less for mDCAT compared with VMAT. This can be attributed to the moderate-to-no modulation for mDCAT (with SSO and without SSO, respectively) compared with the high degree of modulation for VMAT.

An example of the dose difference maps and DVHs for a lung SBRT patient for the extreme amplitude tumour motion without setup errors is shown in Figure 7.9. The behavior of the DVH curves for the GTV for mDCAT and VMAT reflects the changes in the plan quality metrics presented in Table 7.2. The improvement in robustness with the use of SSO in mDCAT compared to mDCAT without SSO can be observed in the reduced deviation on the DVH curve of the GTV. In addition to the GTV dose differences due to motion, larger complementary dose difference to the surrounding healthy tissues just outside the superior and inferior borders of the beam segments are observed (up to $20 \%$ of $\mathrm{Rx}$ ), which are caused by the dose smearing due to tumour motion.

For the typical amplitude of tumour motion, Table 7.2 shows that the magnitude of changes in the plan quality metrics was lower compared with the changes for the extreme tumour motion. The deviation in GTV coverage for mDCAT and VMAT was found to be comparable, with average changes in the metrics being within one percent. The change in $\mathrm{D}_{95 \%}$ for the GTV was very small regardless of the technique, which indicates that the GTV coverage was very similar without and with motion. The GTVto-ITV margin was sufficient to ensure that the GTV was covered in the presence of breathing motion, which has been previously demonstrated in the literature for VMAT deliveries for lung SBRT. ${ }^{107}$ The change in $\mathrm{D}_{2 \%}$ was slightly higher for VMAT. This show that even with small perturbations in the dose distribution, such as for tumour motion with typical amplitude, the high dose volumes (e.g., $\mathrm{D}_{2} \%$ ) for highly modulated 
treatments such as those treated with VMAT could vary due to the steep gradients and the non-uniform photon fluences generated in VMAT treatments.

\subsubsection{Multiple starts of the breathing cycle}

Examples of the results of the interplay effect with five different starts of the breathing cycle are shown in Figures 7.10 and 7.11. The figures contain DVHs for three representative lung SBRT patients to demonstrate the effect of the tumour size and tumour location on the observed change in plan quality when the interplay effect is modelled. For the interplay effect on the DVH, better robustness is manifested in two ways: smaller relative offset of the five DVHs with different starts of the breathing cycle from the original DVH without motion, and smaller spread of the five DHVs among each other.

For the extreme amplitude of tumour motion, shown in Figure 7.10, for mDCAT plans the "effective" offset of the five DVHs with motion against the original DVH was consistent among different patients (consistently an under-dosage), which is due to the fact that mDCAT segments are mostly open around the target. For VMAT, the effective offset of the five DVHs against the original DVH was quite variable from patient to patient, which is due to the interplay between the patient anatomy (tumour size and location), the complex shapes of the segments in the treatment plan, and the breathing pattern (amplitude and period). The spread among the five DVHs with different starts of the breathing cycle for the mDCAT with SSO was minimal, and the results for mDCAT with SSO were very similar (not shown in Figure 7.10). On the other hand, for VMAT there were noticeable differences in the GTV curves for different starts of the breathing cycle. 
For the typical amplitude of tumour motion, shown in Figure 7.11, the DVHs for the GTV after the introduction of tumour motion for the five different starting phases were very close to the DVH of the GTV without motion for both mDCAT and VMAT. VMAT demonstrated a marginal spread of the DVH between the five different starting breathing phases. Overall, this behavior of the GTV coverage indicates that the internal margin used to account for the physiological changes during treatment was sufficient to deliver the intended dose to the tumour, which has been demonstrated previously in the literature for VMAT deliveries for lung SBRT. ${ }^{107}$ 
Table 7.2: Changes in the values of plan quality metrics and their standard deviations for the twelve lung SBRT patients included in this study after introducing extreme and typical amplitudes of tumour motion and modelling the interplay between the plan delivery and the tumour motion, without and with setup error. The extreme amplitude used in this study is $1 \mathrm{~cm}$, and the typical amplitude is the individual GTV-to-ITV margin for each patient (about $3 \mathrm{~mm}$ ). The metrics are evaluated for the GTV.

\begin{tabular}{|c|c|c|c|c|c|}
\hline \multirow{3}{*}{ Metric } & \multirow{3}{*}{ Technique } & \multicolumn{4}{|c|}{ Change in metric } \\
\hline & & \multicolumn{2}{|c|}{$\begin{array}{l}\text { Without setup error } \\
\quad(\text { Section } 7.5)\end{array}$} & \multicolumn{2}{|c|}{$\begin{array}{c}\text { With a } 5 \text { mm setup error } \\
\text { (Section } 7.6)\end{array}$} \\
\hline & & $\begin{array}{l}\text { Extreme } \\
\text { amplitude }\end{array}$ & $\begin{array}{c}\text { Typical } \\
\text { amplitude }\end{array}$ & $\begin{array}{c}\text { Extreme } \\
\text { amplitude }\end{array}$ & $\begin{array}{c}\text { Typical } \\
\text { amplitude }\end{array}$ \\
\hline $\begin{array}{l}D_{95 \%} \\
\text { relative to } \mathrm{Rx}, \\
\text { in } \%\end{array}$ & $\begin{array}{l}\text { mDCAT w/o SSO } \\
\text { mDCAT w/SSO } \\
\text { VMAT }\end{array}$ & $\begin{array}{l}-2.7 \pm 1.7 \\
-1.9 \pm 1.1 \\
-0.4 \pm 2.1\end{array}$ & $\begin{array}{l}-0.3 \pm 0.4 \\
-0.4 \pm 0.5 \\
0.1 \pm 0.6\end{array}$ & $\begin{array}{l}-5.1 \pm 3.1 \\
-5.8 \pm 2.5 \\
-4.8 \pm 5.1\end{array}$ & $\begin{array}{l}-1.8 \pm 1.8 \\
-3.6 \pm 2.7 \\
-2.9 \pm 4.6\end{array}$ \\
\hline $\begin{array}{l}D_{2 \%} \\
\text { relative to } \mathrm{Rx}, \\
\text { in } \%\end{array}$ & $\begin{array}{l}\text { mDCAT w/o SSO } \\
\text { mDCAT w/SSO } \\
\text { VMAT }\end{array}$ & $\begin{array}{l}-0.9 \pm 0.3 \\
-1.0 \pm 1.0 \\
-1.8 \pm 2.7\end{array}$ & $\begin{array}{l}-0.2 \pm 0.2 \\
-0.3 \pm 0.3 \\
-0.8 \pm 1.0\end{array}$ & $\begin{array}{l}-1.1 \pm 0.5 \\
-1.3 \pm 1.4 \\
-1.9 \pm 2.7\end{array}$ & $\begin{array}{l}-0.3 \pm 0.4 \\
-0.4 \pm 0.6 \\
-0.5 \pm 1.3\end{array}$ \\
\hline $\begin{array}{l}\text { Mean dose } \\
\text { relative to } \mathrm{Rx} \text {, } \\
\text { in } \%\end{array}$ & $\begin{array}{l}\text { mDCAT w/o SSO } \\
\text { mDCAT w/SSO } \\
\text { VMAT }\end{array}$ & $\begin{array}{l}-1.4 \pm 0.6 \\
-1.3 \pm 0.9 \\
-0.4 \pm 2.3\end{array}$ & $\begin{array}{l}-0.2 \pm 0.2 \\
-0.4 \pm 0.3 \\
-0.3 \pm 0.5\end{array}$ & $\begin{array}{l}-2.2 \pm 1.1 \\
-2.6 \pm 1.6 \\
-1.7 \pm 3.0\end{array}$ & $\begin{array}{l}-0.7 \pm 0.6 \\
-1.4 \pm 1.3 \\
-1.1 \pm 2.4\end{array}$ \\
\hline CI & $\begin{array}{l}\text { mDCAT w/o SSO } \\
\text { mDCAT w/SSO } \\
\text { VMAT }\end{array}$ & $\begin{array}{l}0.04 \pm 0.02 \\
0.04 \pm 0.02 \\
0.05 \pm 0.02\end{array}$ & $\begin{array}{l}0.00 \pm 0.00 \\
0.00 \pm 0.00 \\
0.00 \pm 0.01\end{array}$ & $\begin{array}{l}0.04 \pm 0.02 \\
0.04 \pm 0.01 \\
0.04 \pm 0.02\end{array}$ & $\begin{array}{l}0.00 \pm 0.01 \\
0.00 \pm 0.01 \\
0.00 \pm 0.01\end{array}$ \\
\hline GI & $\begin{array}{l}\text { mDCAT w/o SSO } \\
\text { mDCAT w/ SSO } \\
\text { VMAT }\end{array}$ & $\begin{array}{l}1.3 \pm 0.7 \\
1.5 \pm 1.1 \\
1.4 \pm 0.7\end{array}$ & $\begin{array}{l}0.1 \pm 0.1 \\
0.1 \pm 0.1 \\
0.1 \pm 0.1\end{array}$ & $\begin{array}{l}1.3 \pm 0.6 \\
1.3 \pm 0.7 \\
1.4 \pm 0.6\end{array}$ & $\begin{array}{l}0.1 \pm 0.1 \\
0.1 \pm 0.1 \\
0.1 \pm 0.2\end{array}$ \\
\hline $\begin{array}{l}\text { Healthy lungs } \\
\mathrm{V}_{20 G y}\end{array}$ & $\begin{array}{l}\text { mDCAT w/o SSO } \\
\text { mDCAT w/SSO } \\
\text { VMAT }\end{array}$ & $\begin{array}{l}-0.20 \pm 0.10 \\
-0.20 \pm-0.20 \\
-0.17 \pm 0.97\end{array}$ & $\begin{array}{l}0.00 \pm 0.03 \\
0.01 \pm 0.05 \\
0.01 \pm 0.05\end{array}$ & $\begin{array}{l}-0.17 \pm 0.32 \\
-0.18 \pm 0.33 \\
-0.19 \pm 0.30\end{array}$ & $\begin{array}{l}0.01 \pm 0.34 \\
0.01 \pm 0.35 \\
0.02 \pm 0.28\end{array}$ \\
\hline
\end{tabular}



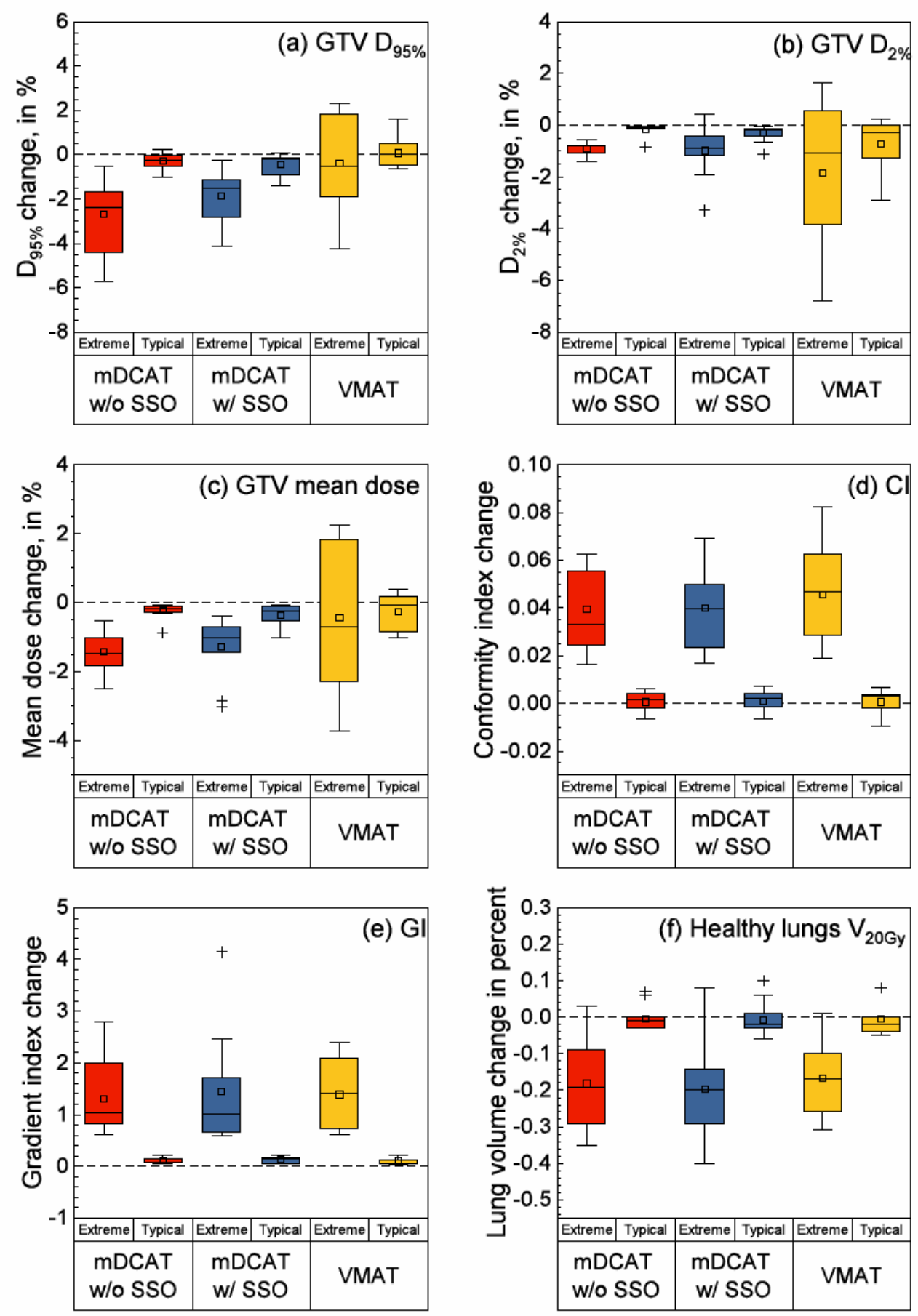

Figure 7.8: Same as in Figure 7.2, but with modelling the interplay between the plan delivery and the tumour motion, rather than isocentre moves only. The results are for the interplay case where the start of the breathing cycle coincides with the start of the treatment delivery and the setup errors are zero. The breathing period is 6 seconds. 

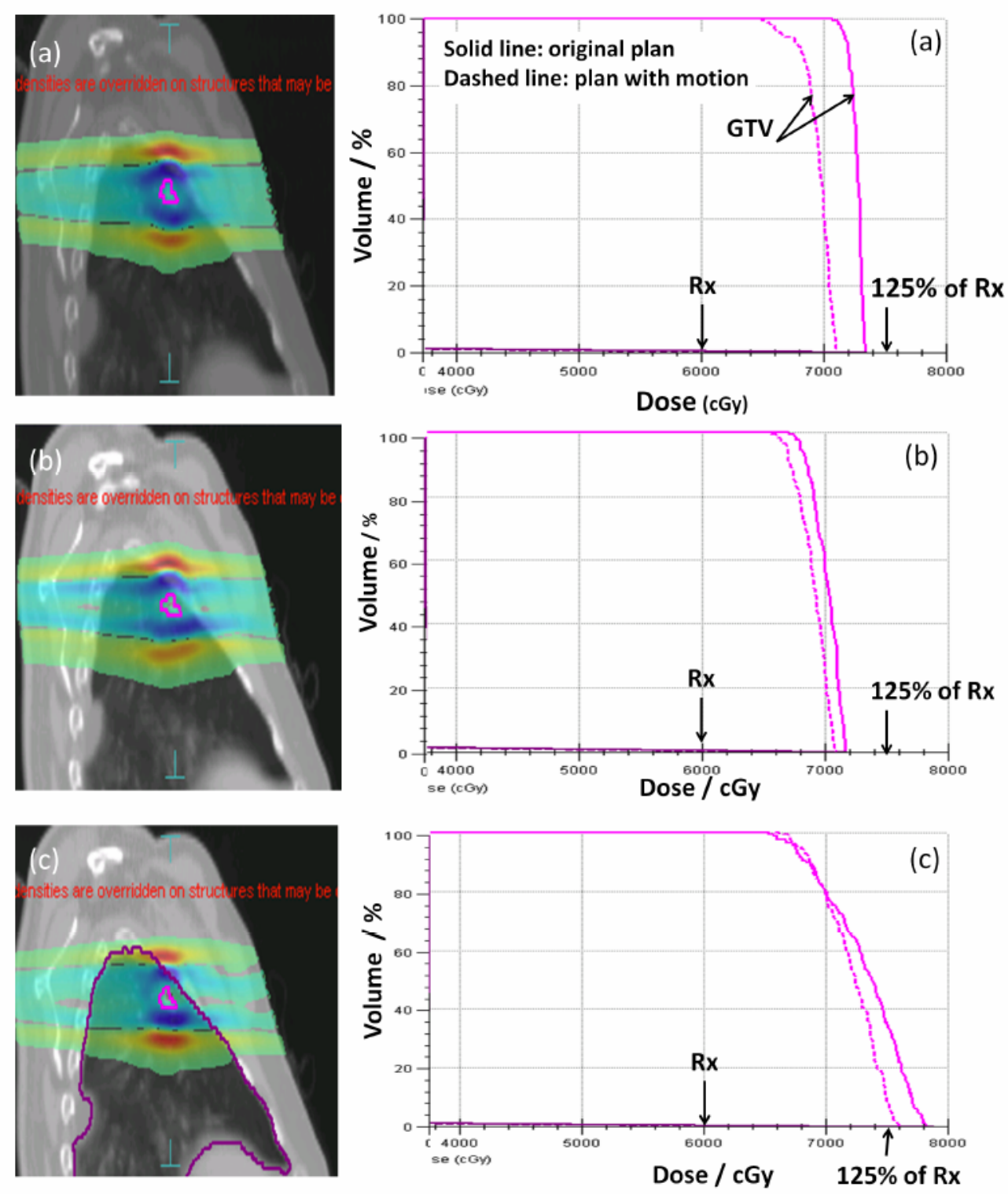

Figure 7.9: With the interplay between the plan delivery and the tumour motion modelled for one start of the breathing cycle, the figure shows for an example lung SBRT patient the dose difference maps in the sagittal view (left) between the plan with the modelled extreme amplitude tumour motion (i.e., $1 \mathrm{~cm}$ ) and the original plan without motion for (a) mDCAT without SSO, (b) mDCAT with SSO, and (c) VMAT. The magenta contour in the sagittal view is the GTV. The scale between the dark red and the dark blue color-washes is from $+20 \%$ to $-20 \%$ of $\mathrm{Rx}$, respectively. In addition to the GTV dose differences due to motion, larger dose difference to the surrounding healthy tissues in the superior-inferior direction are observed. The right panels show the the corresponding DVHs for the GTV with and without tumour motion. The $\mathrm{Rx}$ is $6000 \mathrm{cGy}$, and the GTV typically receives up to $125 \%$ of $\mathrm{Rx}$ (i.e., 7500 cGy). The breathing period is 6 seconds. 

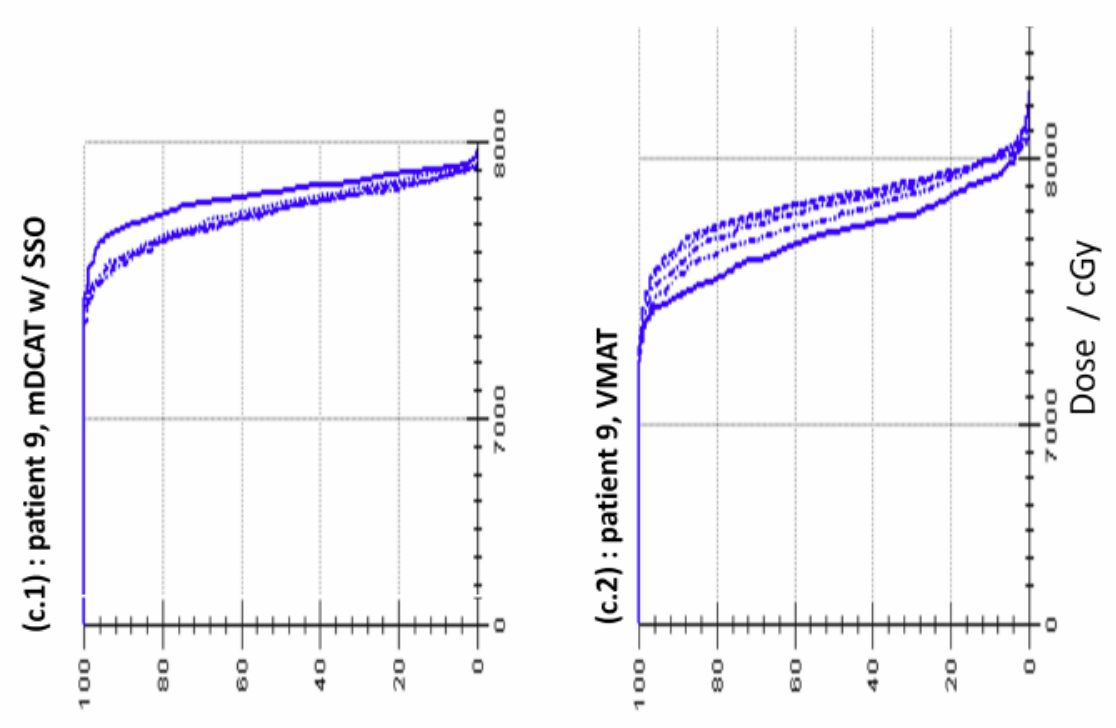

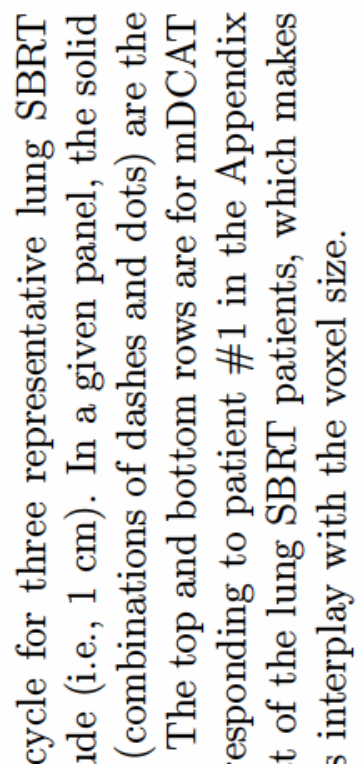
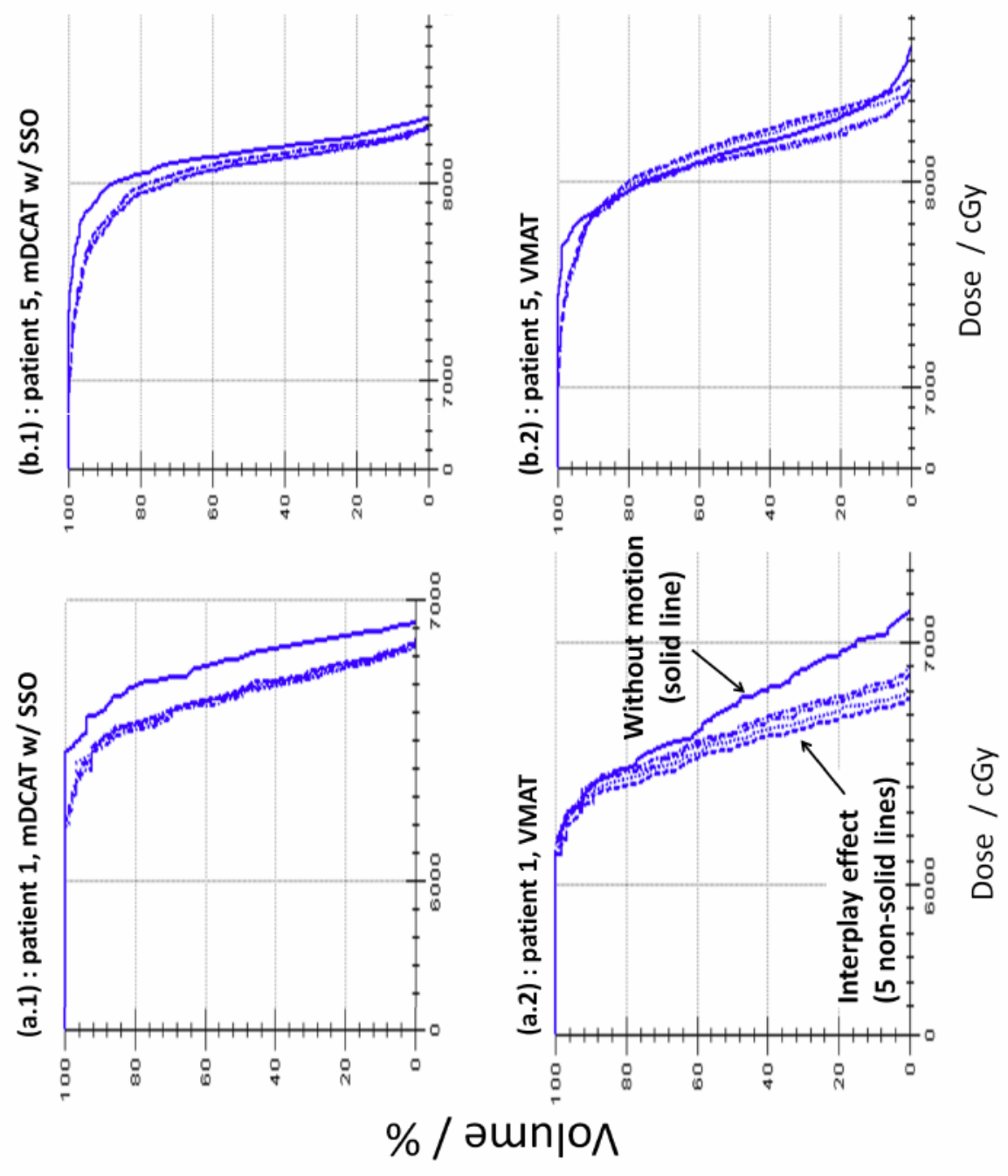

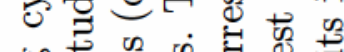

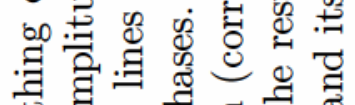

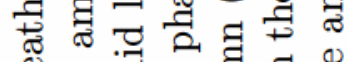
리유 ๑ 苛

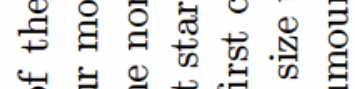

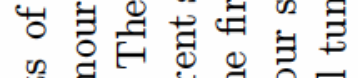

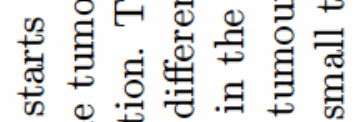

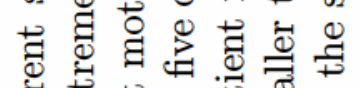

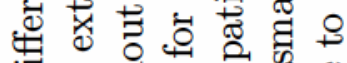
:

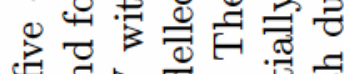

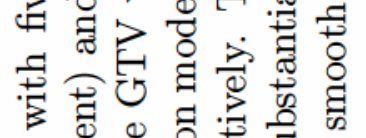

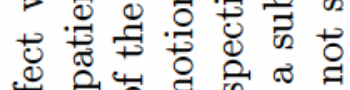

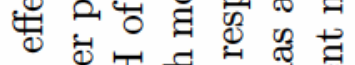

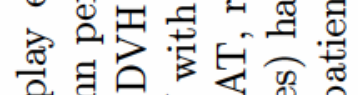

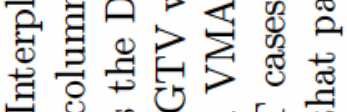
E o 0 近

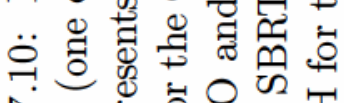

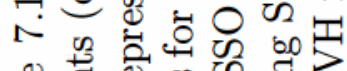

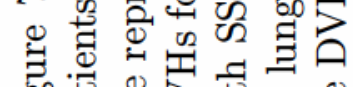

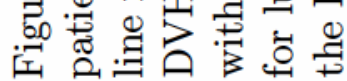



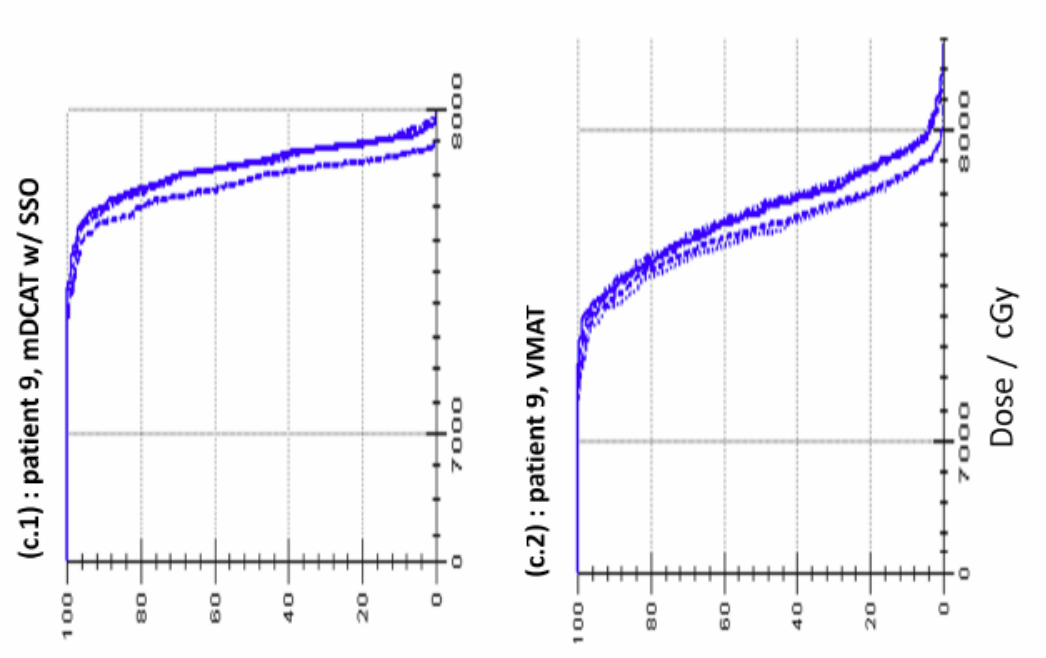

苛

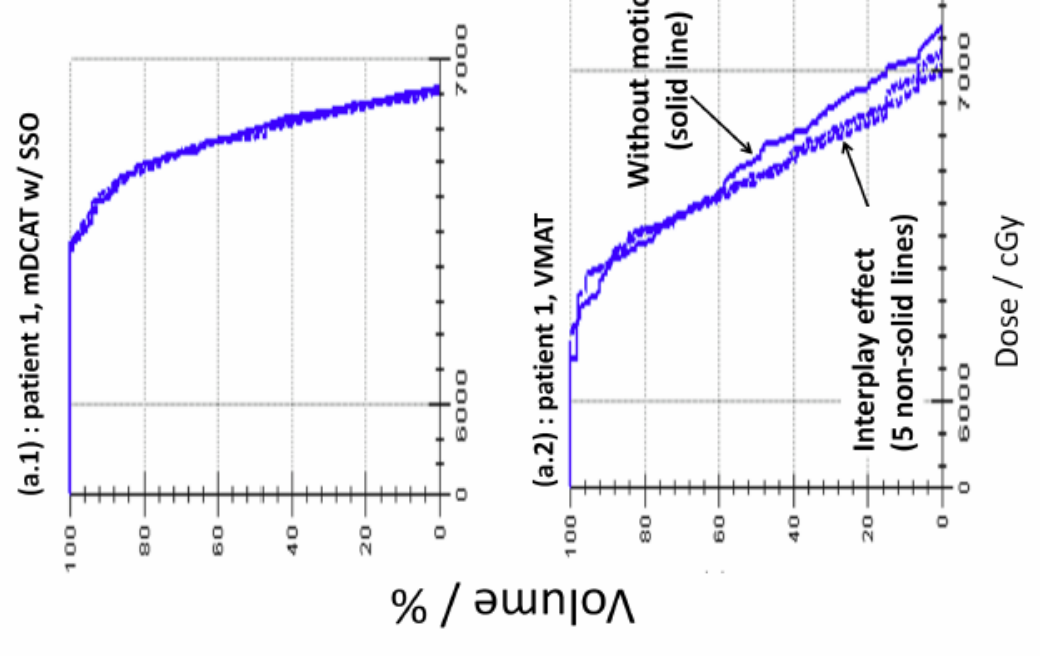

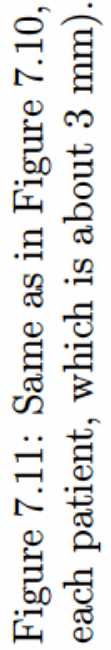




\subsection{Combining interplay effect with setup errors}

Patient setup errors are typically minimized by using immobilization devices during CT simulation and treatment, and by acquiring verification x-ray images (planar 2D images or CBCT images) immediately before treatment to ensure the reproducibility of the patient position between the CT simulation and the delivery. In this study, an example of a setup error was modelled by artificially introducing a plausible onedimensional setup error of $5 \mathrm{~mm}$ in the left-right direction in the mDCAT and VMAT plans of the twelve lung SBRT patients. The magnitude of $5 \mathrm{~mm}$ was chosen since this was the typical ITV-to-PTV margin (i.e., the setup margin) for the lung SBRT cases included in this study. The left-right direction was chosen such that it is different from the superior-inferior direction where most of the breathing motion occurs, in order to allow for more diverse sampling of patient uncertainties. The setup error was modelled on top of modelling the interplay effect due to breathing motion that was discussed in Section 7.4 by shifting the treatment plan isocentre for each sub-plan $5 \mathrm{~mm}$ left from its true position for the twelve lung SBRT patients (in addition to the superior-inferior shift that models the breathing motion for each sub-plan).

The results for combining a setup error with breathing motion for the case where the start of the breathing cycle coincides with the start of the treatment delivery for the twelve lung SBRT patients are shown in Table 7.2 (the two columns with setup error) and Figure 7.12 for the breathing period of 6 seconds. From Table 7.2, it can be seen that the average percent dose error in the GTV $D_{95 \%}$ was substantially higher in

the presence of a setup error versus without a setup error for both extreme and typical amplitudes of breathing motion, and regardless of the technique. From Figure 7.12, the spread of the dose errors among patients was higher for VMAT versus mDCAT, 
showing the same behavior as that without the setup error. Combining a setup error with breathing motion increases the changes in plan quality metrics compared to their counterparts without the error.

The interplay effect using the combination of a setup error and five different starts of the breathing cycle is shown in Figures 7.13 and 7.14 for the breathing period of 6 seconds. Combining a setup error with breathing motion causes larger deviations that can become clinically significant depending on the patient details. 

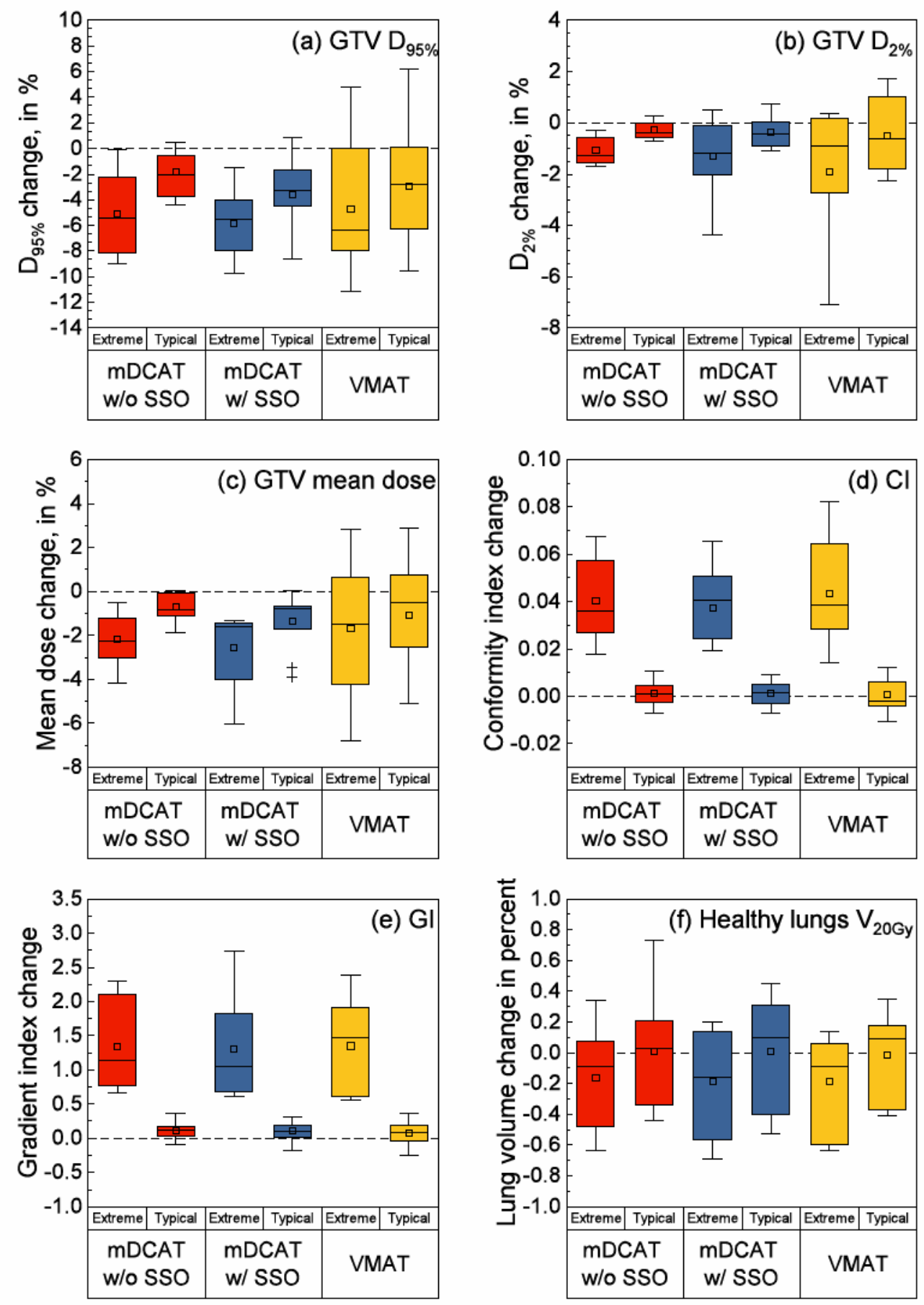

Figure 7.12: Same as in Figure 7.8, but combined with a $5 \mathrm{~mm}$ setup error in the left-right direction. 

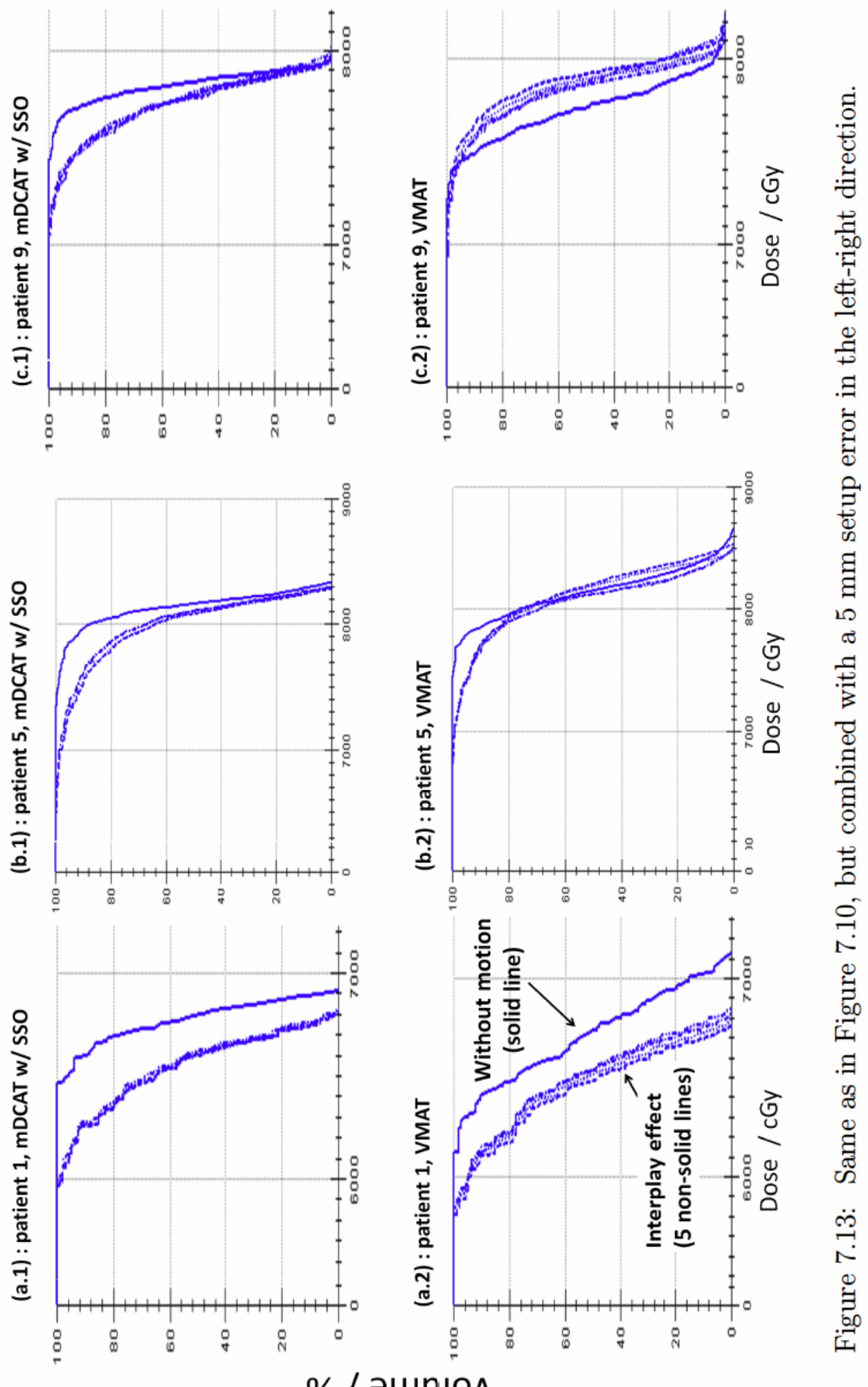

$\%$ / əun|O^ 

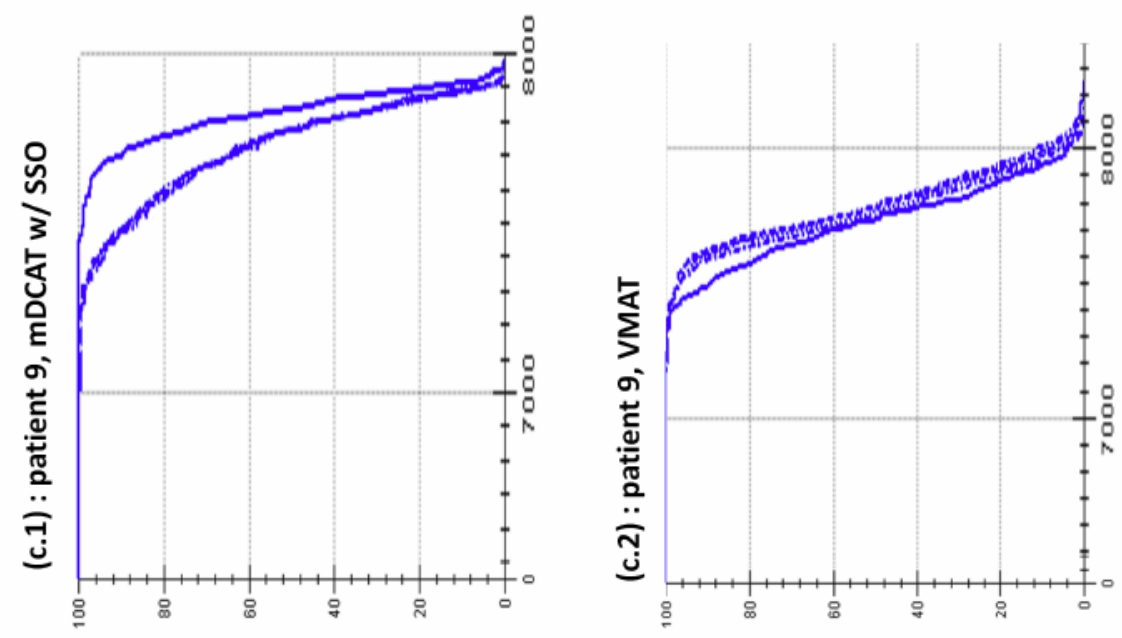

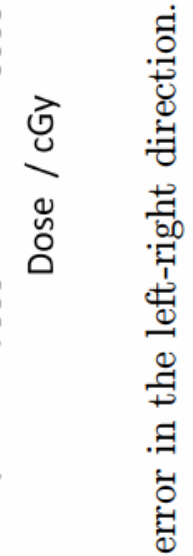
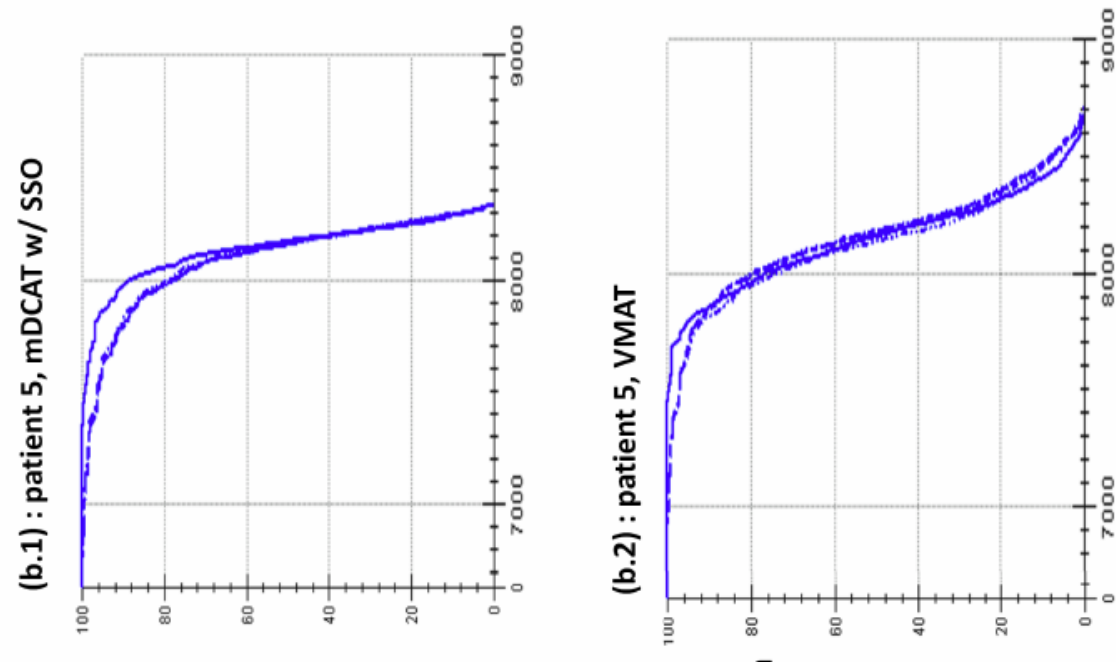

䓌
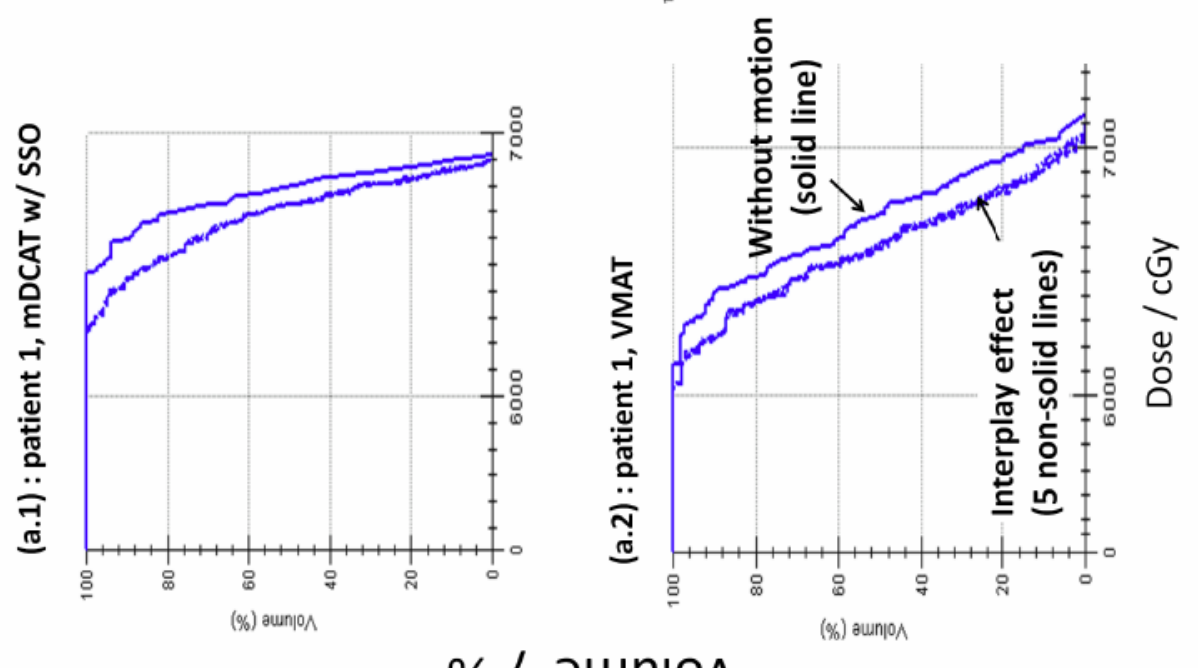

\% / әயпюо 


\subsection{Discussion and summary}

Both VMAT and mDCAT were found to be robust in terms of target coverage at the $1 \%$ level against typical tumour motion uncertainties. The internal margin added to the GTV to account for the physiological changes during treatment, such as breathing motions, is sufficient. The variability in the resulting changes in plan quality metrics for VMAT over a cohort of patients is more pronounced than that for mDCAT because of the complex segment shapes that VMAT delivery has, and the more dynamic motion of the LINAC components during VMAT delivery. The interplay effects vary among patients depending on factors such as tumour size and location, surrounding OARs, and the magnitude of the patient-specific GTV-to-ITV margin. mDCAT is more predictable against the interplay, but the systematic effect may be more than VMAT, which is caused by the nature of mDCAT plans mostly conforming to the tumour shape during delivery.

The spread of the interplay effect (demonstrated through the five different DVHs) is generally not very large, even for the slower breathing (i.e., the longer breathing period of 6 seconds) combined with extreme motion amplitude, and combined with highly modulated treatments (e.g., VMAT), which can be explained as follows. SBRT treatments are high dose per fraction, therefore the treatment delivery is very slow (compared to conventionally fractionated treatments that offer much lower dose per fraction). The slow delivery of SBRT treatments combined with a sufficient ITV margin makes the normal range of human breathing periods to be not slow enough to have a strong resonance with SBRT deliveries to the point of causes appreciable interplay effect. This also implies that the simpler approach of modelling breathing motion with an isocentre-moves-only (as done in Section 7.2) is sufficiently accurate to scope most 
of the dosimetric impact of tumour motion for lung SBRT patients (however, this needs to be assessed separately for conventional treatments). The slow delivery of SBRT treatments versus the breathing period is one of the reasons the robustness of mDCAT and VMAT is relatively comparable for such treatments, and is clinically acceptable for typical breathing cycles.

Assuming a perfect patient setup, dose distribution blurring due to patient breathing motion can be viewed positively in SBRT treatments because the center of an SBRT plans is typically at a higher dose than the $\mathrm{Rx}$, up to $125 \%$ of $\mathrm{Rx}$, and thus when the tumor moves within the ITV, it periodically gets in and out of the larger dose area, which makes for a more uniform dose due to the motion blurring, and it is more obvious for mDCAT than it is for VMAT because the fields are open around the target for most of the mDCAT delivery whereas the complex segment shapes in VMAT may not support the argument just presented. This argument is less valid when a setup error exists.

The results for the 3 second breathing period (not shown) were comparable to the results for the 6 second period presented throughout Sections 7.5 and 7.6, with the spread of the interplay effect being marginally smaller for the shorter breathing period of 3 seconds.

In summary, for lung SBRT patients with a suitable ITV margin, modelling tumour motion in the superior-inferior direction due to breathing showed that for typical motion amplitude, mDCAT and VMAT are equally robust in terms of target coverage at the $1 \%$ level, with tumour coverage largely unaffected by the motion. For extreme motion amplitude (i.e., $1 \mathrm{~cm}$ ), VMAT is more robust compared to mDCAT in terms of systematic dose deviations. However, VMAT has higher tendency for variability in the results among patients, and thus the interplay effect can be more obvious in VMAT for 
particular patients. Combining systematic setup errors with tumour motion can lead to deviations that are clinically relevant, depending on the patient anatomy and treatment plan details. 


\section{Chapter 8}

\section{Summary, conclusions, and future work}

Summary. A new implementation of DCAT (mDCAT) in the Monaco TPS allows for variable dose rate between arc segments as well as moderate modulation around the target periphery, which is different from its classic version that only allows for constant dose rate and the radiation beams are strictly shaped around the target without any modulation. Generating an mDCAT plan in the Monaco TPS requires the use of inverse planning optimization. In this study, mDCAT was evaluated in terms of plan optimization efficiency, plan quality, and plan robustness against machine and patient-related uncertainties, which are three of the main considerations when choosing a technique for radiation treatment. The performance of mDCAT was compared to two of the most commonly used RT techniques: (1) 3DCRT, a forward planning technique commonly used for relatively simpler treatments, and, (2) VMAT, a technique known for its high degree of modulation which allows for very conformal radiation dose distributions, but is more demanding in plan optimization and more prone to being impacted by machine and patient uncertainties.

Results showed that mDCAT is two to three times more efficient in terms of treatment plan optimization compared to VMAT. In terms of absolute times, the optimization time can be reduced to under three minutes for mDCAT using a clinically acceptable set of calculation parameters. The average treatment delivery times for $\mathrm{mD}$ CAT and VMAT are short, and so the absolute gains of treating with mDCAT versus 
VMAT are small. Efficient optimization and efficient treatment delivery become clinically relevant for rapid-access palliative situations where the process of scan-plan-treat is to be done in a typical treatment time slot of around 30 minutes. The number of MUs delivered with mDCAT (without and with SSO with similar number of MUs) is less compared to that for VMAT deliveries.

In terms of plan quality, results show that for large targets such as those with palliative spine treatments, mDCAT performs midway between the clinically acceptable 3DCRT and VMAT plans, with a slight improvement for mDCAT with SSO versus without SSO. For small targets such as those for stereotactic treatments, the plan quality achievable with VMAT and mDCAT are very comparable. 3DCRT is not used for stereotactic treatments in general, and hence it was not compared with mDCAT in terms of plan quality for small targets.

mDCAT was shown to be more robust against machine uncertainties compared to VMAT, regardless of the magnitude of the machine errors. For extreme machine errors such as an MLC positional error at the tolerance level of $2 \mathrm{~mm}$, the loss of target coverage is limited to about $2 \%$ for mDCAT, compared to $5 \%$ for VMAT. For machine errors within the range observed in clinical treatments, the changes in target coverage are within one percent for both mDCAT and VMAT. The addition of SSO slightly worsens the robustness of mDCAT compared to that without SSO due to the added modulation. However, mDCAT with SSO is still more robust compared to VMAT.

In terms of robustness against patient-related uncertainties such as breathing motion, for typical motion amplitude, mDCAT and VMAT were found to be equally robust at the $1 \%$ level for target coverage. For extreme motion amplitude (i.e., $1 \mathrm{~cm}$ ), VMAT was slightly more robust compared to mDCAT in terms of systematic dose deviations, however, VMAT has higher tendency for outliers and wider range of dosimetric changes 
among patients, and thus the interplay effect can be more obvious in VMAT for particular patients. Combining systematic setup errors with tumour motion can lead to deviations that are clinically relevant, depending on the patient anatomy and treatment plan details.

Conclusions. The different characteristics of mDCAT found in this study can be useful for a number of clinical situations. The faster plan optimization of mDCAT compared to VMAT with an added dose conformity (compared to 3DCRT) can be integrated with the recent models for rapid access palliation, which aim to execute the whole EBRT process within a typical treatment time slot (i.e., less than one hour) by using the imaging devices in the treatment unit (i.e., CBCT). Current rapid-access palliation models use 3DCRT as the treatment technique for the purpose of faster treatment planning. Reducing the optimization time to under 3 minutes with mDCAT (versus the slower VMAT optimization), combined with the large improvement in target dose conformality for mDCAT (versus 3DCRT) makes the use of mDCAT a big step towards higher-quality rapid-access palliation. With the plan quality achievable with mDCAT for very small targets (i.e., lung SBRT, brain with small target), mDCAT can be an alternative technique for stereotactic treatments in institutions that do not have dedicated machines (i.e., CyberKnife) and can be a more robust treatment technique option particularly for very small treatment fields. In general, mDCAT is a good addition to the available treatment techniques given that it only requires minimal overhead to implement clinically.

Future work. The following are extensions of the work presented in this study. They also serve as a summary of the limitations and approximations in this study. Although the work presented in this study is specific to the Monaco TPS by Elekta, many elements of 
this work that characterized the behaviour of mDCAT can be used as starting ground for future works that involve other implementations of DCAT by other vendors.

- Standardization of sets of objectives and constraints for disease sites where mDCAT provides an advantage as an intermediate solution between 3DCRT and VMAT. This is to facilitate minimal user intervention which will allow for close to real-time inverse planning optimization with mDCAT.

- More refined investigation of TP degrees of freedom (noncoplanar beams, arc limits, jaw positions, collimator angle, etc) to evaluate further improvement in mDCAT plan quality. Maximizing the degrees of freedom by allowing different combinations of the machine components can extend the application of mDCAT to disease sites other than those mentioned in this study.

- More detailed robustness analysis for different machine parameters beyond MLCs. The machine parameters to be explored can include couch, gantry, and jaws. Actual machine component offsets from the treatment delivery log files can be introduced to the treatment plans to calculate the actual deviations from the cumulative effect of these different machine component offsets.

- Investigating the impact of robustness against the interplay effect for healthy tissues in the immediate vicinity of the target, not only in terms of target coverage. Additional metrics can be used to evaluate the changes in plan quality, both in target coverage and in critical organ doses.

- More accurate modelling of patient breathing motion and its interplay with the plan delivery, including patient deformations and accurate dose accumulation using the $4 \mathrm{DCT}$ information. 
- Experimental dosimetric assessment of the beam model in the TPS for mDCAT versus the established current techniques (i.e., 3DCRT and VMAT). This assessment involves output factor measurements for different static fields (different sizes and shapes) and for arc deliveries, and using different detectors (e.g., 3D detector arrays, reference ion chambers, Gafchromic films). The results of this would not mean that one technique is more accurate over the other against experiments, but would rather informs refinements in the beam model to best suit all the techniques that use the beam model if mDCAT is to be added to the repertoire of available treatment techniques. 


\section{Appendix: Anonymized list of patients}

Throughout the appendix, the blue (inner) contour is the CTV for spine, ITV for lung, and GTV for brain. The red (outer) contour is the PTV.

\section{A. Patient list for the palliative spine cases}

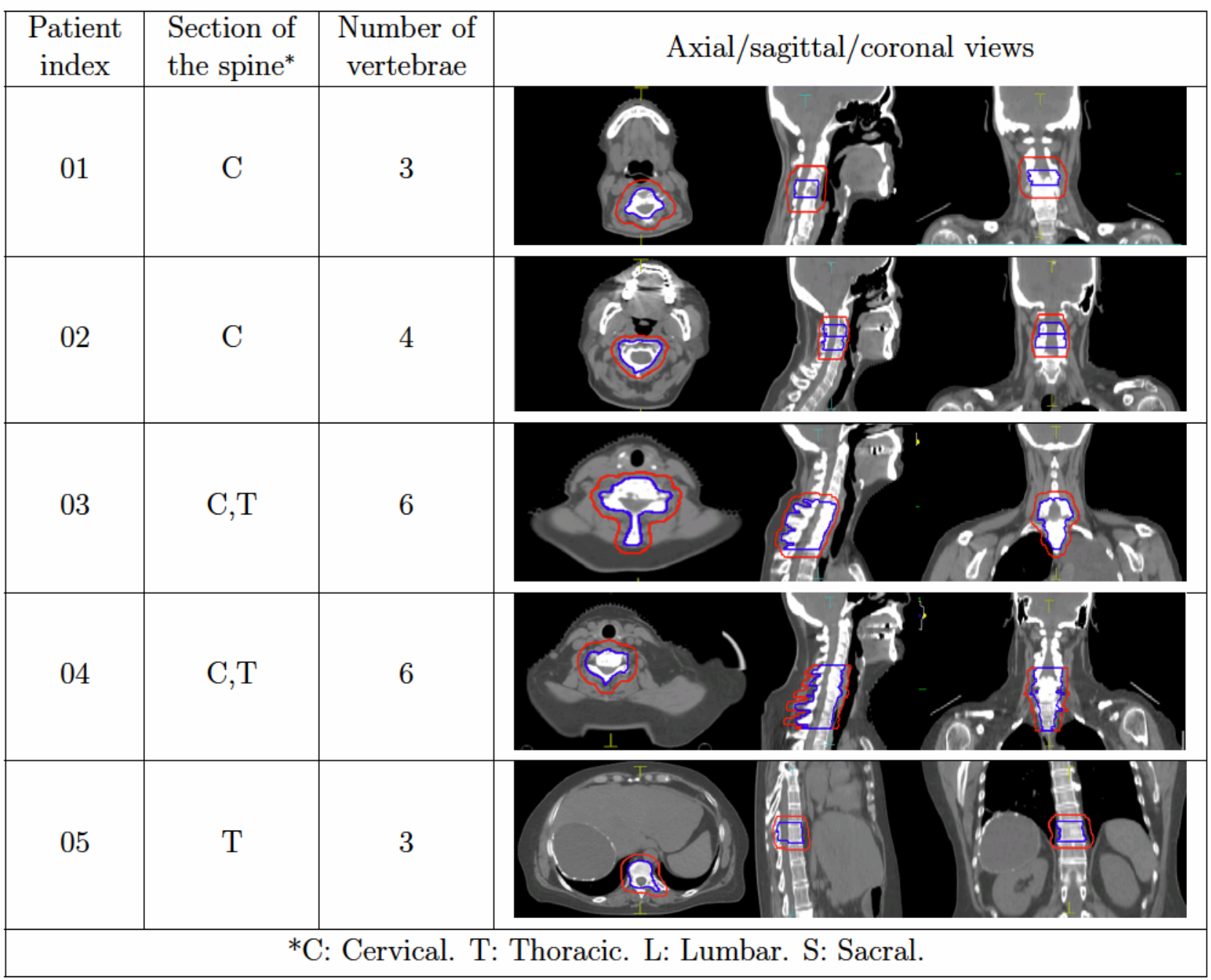




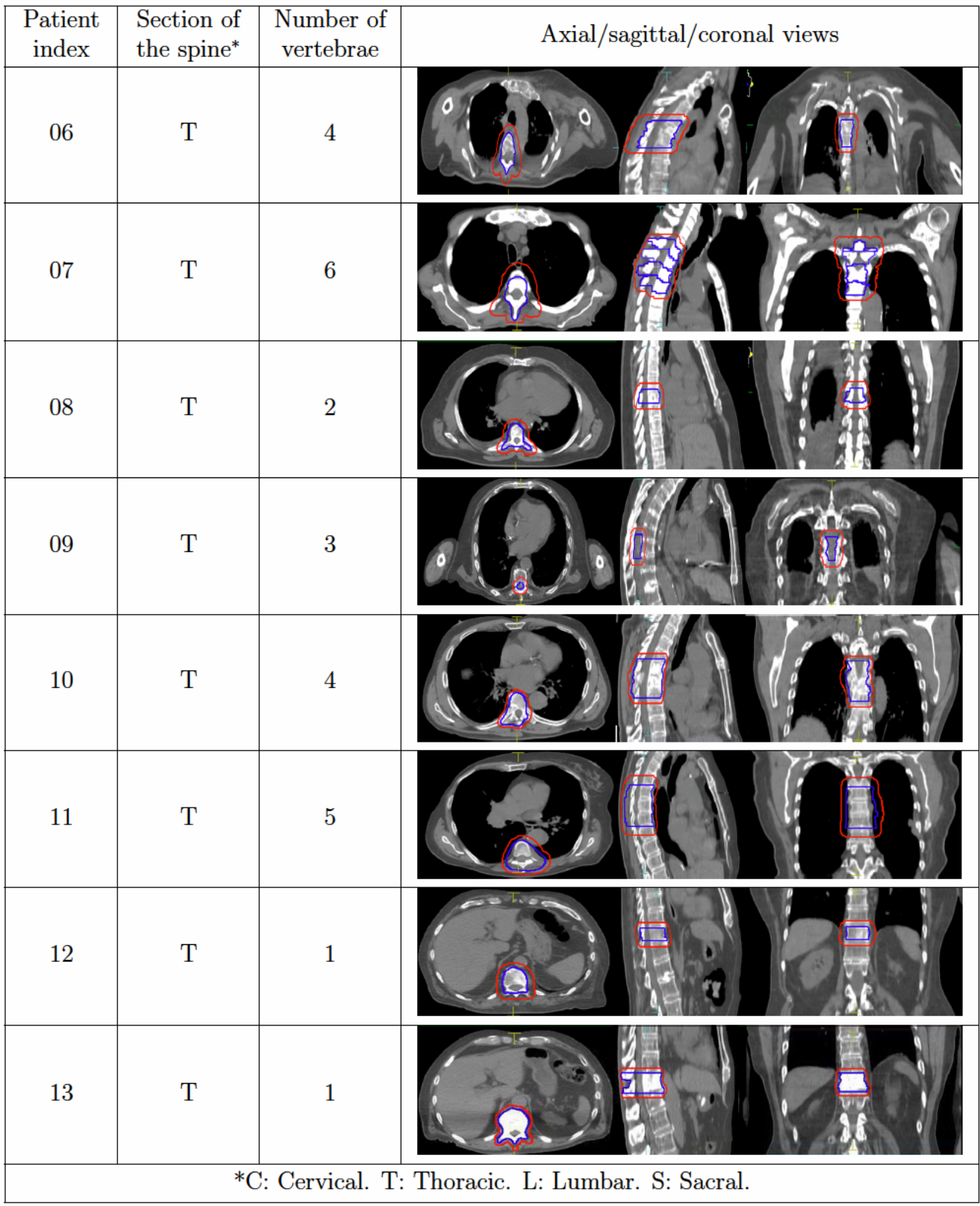




\begin{tabular}{|c|c|c|c|}
\hline $\begin{array}{l}\text { Patient } \\
\text { index }\end{array}$ & $\begin{array}{l}\text { Section of } \\
\text { the spine* }\end{array}$ & $\begin{array}{l}\text { Number of } \\
\text { vertebrae }\end{array}$ & Axial/sagittal/coronal views \\
\hline 14 & $\mathrm{~T}$ & 4 & \\
\hline 15 & $\mathrm{~T}$ & 8 & \\
\hline 16 & $\mathrm{~T}$ & 9 & \\
\hline 17 & $\mathrm{~T}, \mathrm{~L}$ & 4 & \\
\hline 18 & $\mathrm{~T}, \mathrm{~L}$ & 6 & \\
\hline 19 & $\mathrm{~L}$ & 2 & \\
\hline 20 & $\mathrm{~L}$ & 4 & \\
\hline & & Cervical. T & umbar. S: Sacral. \\
\hline
\end{tabular}




\begin{tabular}{|c|c|c|c|}
\hline $\begin{array}{c}\text { Patient } \\
\text { index }\end{array}$ & $\begin{array}{l}\text { Section of } \\
\text { the spine* }\end{array}$ & $\begin{array}{c}\text { Number of } \\
\text { vertebrae }\end{array}$ & Axial/sagittal/coronal views \\
\hline 21 & $\mathrm{~L}$ & 3 & \\
\hline 22 & $\mathrm{~L}, \mathrm{~S}$ & 2 & \\
\hline 23 & $\mathrm{~L}, \mathrm{~S}$ & 4 & \\
\hline 24 & $\mathrm{~L}, \mathrm{~S}$ & 8 & \\
\hline 25 & $\mathrm{~S}$ & 4 & \\
\hline & & Cervical. T & ar. S: Sacral. \\
\hline
\end{tabular}


B. Patient list for the lung SBRT cases

\begin{tabular}{|c|c|c|}
\hline $\begin{array}{c}\text { Patient } \\
\text { index }\end{array}$ & $\begin{array}{l}\text { PTV volume } \\
\text { in } \mathrm{cm}^{3}\end{array}$ & Axial/sagittal/coronal views \\
\hline 01 & 7.4 & \\
\hline 02 & 23.1 & \\
\hline 03 & 12.5 & \\
\hline 04 & 29.8 & \\
\hline 05 & 29.9 & \\
\hline 06 & 15.0 & \\
\hline
\end{tabular}




\begin{tabular}{|c|c|c|}
\hline $\begin{array}{c}\text { Patient } \\
\text { index }\end{array}$ & $\begin{array}{l}\text { PTV volume } \\
\text { in } \mathrm{cm}^{3}\end{array}$ & Axial/sagittal/coronal views \\
\hline 07 & 47.1 & \\
\hline 08 & 18.9 & \\
\hline 09 & 13.6 & \\
\hline 10 & 51.2 & \\
\hline 11 & 16.4 & \\
\hline 12 & 13.1 & \\
\hline
\end{tabular}


C. Patient list for the brain cases

\begin{tabular}{|c|c|c|}
\hline $\begin{array}{l}\text { Patient } \\
\text { index }\end{array}$ & $\begin{array}{c}\text { PTV volume } \\
\text { in } \mathrm{cm}^{3}\end{array}$ & Axial/sagittal/coronal views \\
\hline 01 & 2.1 & \\
\hline 02 & 3.1 & \\
\hline 03 & 3.1 & \\
\hline 04 & 5.1 & \\
\hline 05 & 5.5 & \\
\hline 06 & 6.6 & \\
\hline
\end{tabular}




\begin{tabular}{|c|c|c|}
\hline $\begin{array}{l}\text { Patient } \\
\text { index }\end{array}$ & $\begin{array}{l}\text { PTV volume } \\
\text { in } \mathrm{cm}^{3}\end{array}$ & Axial/sagittal/coronal views \\
\hline 07 & 8.7 & \\
\hline 08 & 11.0 & \\
\hline 09 & 15.3 & \\
\hline 10 & 17.5 & \\
\hline 11 & 19.4 & \\
\hline 12 & 20.4 & \\
\hline
\end{tabular}




\section{References}

[1] H. E. Johns and J. R. Cunningham, The physics of radiology, 4th ed., Charles C. Thomas, Springfield, Illinois, 1983.

[2] E. B. Podgorsak, editor, Review of Radiation Oncology Physics: A Handbook for Teachers and Students, Educational Report Series, IAEA, Vienna, Austria, 2004.

[3] ICRU, Prescribing, recording and reporting photon beam therapy, ICRU Report 50, ICRU, Washington D.C., 1993.

[4] F. M. Khan, The Physics of Radiation Therapy, Lipincott Williams and Wilkins, Baltimore, Maryland, 3rd edition, 2003.

[5] Y. Y. Wang and H. Zhe, Clinical application of multimodality imaging in radiotherapy treatment planning for rectal cancer, Cancer Imaging 13, 495-501 (2013).

[6] D. Thorwarth, Functional imaging for radiotherapy treatment planning: current status and future directions - a review, Brit. J. Radiol. 88, R1 - R9 (2015).

[7] ICRU, Prescribing, recording and reporting photon beam therapy (Supplement to ICRU Report 50), ICRU Report 62, ICRU, Washington D.C., 1999.

[8] P. R. Almond, P. J. Biggs, B. M. Coursey, W. F. Hanson, M. S. Huq, R. Nath, and D. W. O. Rogers, AAPM's TG-51 protocol for clinical reference dosimetry of high-energy photon and electron beams, Med. Phys. 26, 1847 - 1870 (1999).

[9] J. M. Galvin, G. Ezzell, A. Eisbrauch, C. Yu, B. Butler, Y. Xiao, I. Rosen, J. Rosenman, M. Sharpe, L. Xing, P. Xia, T. Lomax, D. A. Low, and J. Palta, Implementing IMRT in clinical practice: a joint document of the American Society for Therapeutic Radiology and Oncology and the American Association of Physicists in Medicine, Int. J. Radiat. Oncol. Biol. Phys. 58, 1616-1634 (2004).

[10] R. L. Stern, R. Heaton, M. W. Fraser, S. M. Goddu, T. H. Kirby, K. L. Lam, A. Molineu, and T. C. Zhu, Verification of monitor unit calculations for nonIMRT clinical radiotherapy: Report of AAPM Task Group 114, Med. Phys. 38, $504-530$ (2011).

[11] J. P. Gibbons, J. A. Antolak, D. S. Followill, M. S. Huq, E. E. Klein, K. L. Lam, J. R. Palta, D. M. Roback, M. Reid, and F. M. Khan, Monitor unit calculations for external photon and electron beams: Report of the AAPM Therapy Physics Committee Task Group No. 71, Med. Phys. 41, 031501-1 - 031501-34 (2014). 
[12] M. D. Kumar, N. Thirumavalavan, and M. Babaiah, QA of intensity-modulated beams using dynamic MLC log files, J. Med. Phys. 31, 36-41 (2006).

[13] M. Joiner and A. van der Kogel, Basic clinical radiobiology, 4th ed., Hodder Arnold, Great Britain, 2009.

[14] R. D. Timmerman, An overview of hypofractionation and introduction to this issue of Seminars in Radiation Oncology, Semin. Radiat. Oncol. 18, 215-222 (2008).

[15] B. K. Chang and R. D. Timmerman, Stereotactic body radiation therapy: a comprehensive review, Am. J. Clin. Oncol. 30, 637-644 (2007).

[16] N. Trakul, A. C. Koong, and D. T. Chang, Stereotactic body radiotherapy in the treatment of pancreatic cancer, Semin. Radiat. Oncol. 24, 140-147 (2014).

[17] P. R. Anderson and L. R. Coia, Fractionation and outcomes with palliative radiation therapy, Semin. Radiat. Oncol. 10, 191-199 (2000).

[18] J. F. Fowler, 21 years of biologically effective dose, Brit. J. Radiol. 83, 554-568 (2010).

[19] R. Mohan, Q. Wu, M. Manning, and R. Schmidt-Ullrich, Radiobiological considerations in the design of fractionation strategies for intensity-modulated radiation therapy of head and neck cancers, Int. J. Radiat. Oncol. Biol. Phys. 46, 2554-568 (2000).

[20] J. F. Fowler, W. A. Tomé, and J. D. Fenwick, A challenge to traditional radiation oncology, Int. J. Radiat. Oncol. Biol. Phys. 60, 1241-1256 (2004).

[21] C. B. Saw, R. Yakoob, C. A. Enke, T. P. Lau, and K. M. Ayyangar, Immobilization devices for intensity-modulated radiation therapy (IMRT), Med. Dos. 26, 71-77 (2001).

[22] K. M. Langen and D. T. L. Jones, Organ motion and its management, Int. J. Radiat. Oncol. Biol. Phys. 50, 265-278 (2001).

[23] P. J. Keall, G. S. Mageras, J. M. Balter, R. S. Emery, K. M. Forster, S. B. Jiang, J. M. Kapatoes, H. D. Kubo, D. A. Low, M. J. Murphy, B. R. Murray, C. R. Ramsey, M. B. V. Herk, S. S. Vedam, J. W. Wong, and E. Yorke, The Management of Respiratory Motion in Radiation Oncology: Report of AAPM Task Group 76, Report 91, AAPM, 2006.

[24] P. J. Keall, G. S. Mageras, J. M. Balter, R. S. Emery, K. M. Forster, S. B. Jiang, J. M. Kapatoes, D. A. Low, M. J. Murphy, B. R. Murray, C. R. Ramsey, M. B. V. Herk, S. S. Vedam, J. W. Wong, and E. Yorke, Respiratory motion in radiation oncology, Med. Phys. 33, $3874-3900$ (2006). 
[25] Q.-S. Chen, M. S. Weinhous, F. C. Deibel, and J. P. Ciezki, Fluoroscopic study of tumor motion due to breathing: facilitating precise radiation therapy for lung cancer patients, Med. Phys. 28, 1850-1856 (2001).

[26] Y. Seppenwoolde, H. Shirato, K. Kitamura, S. Shimizu, M. van Herk, J. V. Lebesque, and K. Miyasaka, Precise and real-time measurement of 3D tumor motion in lung due to breathing and heartbeat, measured during radiotherapy, Int. J. Radiat. Oncol. Biol. Phys. 53, 822-834 (2002).

[27] S. H. Benedict, K. M. Yenice, D. Followill, J. M. Galvin, W. Hinson, B. Kavanagh, P. Keall, M. Lovelock, M. C. Schell, B. Salter, D. J. Schlesinger, A. S. Shiu, T. Solberg, D. Y. song, V. Stieber, R. Timmerman, W. A. Tomé, D. Verellen, and L. Wang, AAPM's TG-101 protocol for stereotactic body radiation therapy, Med. Phys. 37, 4078 - 4101 (2010).

[28] G. T. Y. Chen, J. H. Kung, and K. P. Beaudette, Artifacts in computed tomography of moving objects, Semin. Radiat. Oncol. 14, 19-26 (2004).

[29] P. Keall, 4-Dimensional computed tomography imaging and treatment planning, Semin. Radiat. Oncol. 14, 81-90 (2004).

[30] R. Muirhead, S. G. McNee, C. Featherstone, K. Moore, and S. Muscat, Use of Maximum Intensity Projections (MIP) for target outlining in 4DCT radiotherapy planning, J. Thorac. Oncol. 8, 1433-1438 (2008).

[31] S. Webb, The physical basis of IMRT and inverse planning, Brit. J. Radiol. 76, $678-689$ (2003).

[32] T. Bortfeld, IMRT: a review and preview, Phys. Med. Biol. 51, R363 - R379 (2006).

[33] L. Velderman, I. Madani, F. Hulstaert, G. D. Meerleer, M. Mareel, and W. D. Neve, Evidence behind use of intensity-modulated radiotherapy: a systematic review of comparative clinical studies, Lancet Oncol. 9, 367-375 (2008).

[34] W. D. Neve, W. D. Gersem, and I. Madani, Rational use of intensity-modulated radiation therapy: the importance of clinical outcome, Semin. Radiat. Oncol. 22, 40-49 (2012).

[35] A. Takeda, E. Kunieda, N. Sanuki, T. Ohashi, Y. Oku, Y. Sudo, H. Iwashita, Y. Ooka, Y. Aoki, N. Shigematsu, and A. Kubo, Dose distribution analysis in stereotactic body radiotherapy using dynamic conformal multiple arc therapy, Int. J. Radiat. Oncol. Biol. Phys. 74, 363-369 (2009).

[36] B. M. Rauschenbach, L. Mackowiak, and H. K. Malhotra, A dosimetric comparison of three-dimensional conformal radiotherapy, volumetric-modulated arc therapy, and dynamic conformal arc therapy in the treatment of non-small cell lung cancer using stereotactic body radiotherapy, J of App Clin Med Phys 15, 147-161 (2014). 
[37] X. Ming, Y. Feng, H. Liu, Y. Zhang, L. Zhou, and J. Deng, Cardiac exposure in the dynamic conformal arc therapy, intensity-modulated radiotherapy and volumetric arc therapy of lung cancer, PLoS ONE 10, 147-161 (2015).

[38] R. G. J. Wiggenraad, A. L. Petoukhova, L. Versluis, and J. P. C. van Santvoort, Stereotactic radiotherapy of intracranial tumors: a comparison of intensitymodulated radiotherapy and dynamic conformal arc, Int. J. Radiat. Oncol. Biol. Phys. 24, 1018-1026 (2009).

[39] L. J. Hazard, B. Wang, T. B. Skidmore, S.-S. Chern, B. J. Salter, R. L. Jensen, and D. C. Shrieve, Conformity of linac-based stereotactic radiosurgery using dynamic conformal arcs and micro-multileaf collimators, Int. J. Radiat. Oncol. Biol. Phys. 73, 562-570 (2009).

[40] A. Ernst-Stecken, U. Lambrecht, O. Ganslandt, R. Mueller, and R. Fahlbusch, Radiosurgery of small skull-based lesions, Strahlentherapie und Onkologie 181, 336-344 (2005).

[41] M. Ding, F. Newman, B. D. Kavanagh, K. Stuhr, T. K. Johnson, and L. E. Gaspar, Comparative dosimetric study of three-dimensional conformal, dynamic conformal arc, and intensity-modulated radiotherapy for brain tumor treatment using Novalis system, Int. J. Radiat. Oncol. Biol. Phys. 66, S82-S86 (2006).

[42] T. D. Solberg, K. L. Boedeker, R. Fogg, M. T. Selch, and A. A. F. DeSalles, Dynamic arc radiosurgery field shaping: a comparison with static field conformal and noncoplanar circular arcs, Int. J. Radiat. Oncol. Biol. Phys. 49, 1481-1491 (2001).

[43] T. Lee, P. Chao, C. Wang, J. Lan, Y. Huang, H. Hsu, C. Sung, T. Su, S. Lian, and F. Fang, Dosimetric comparison of helical Tomotherapy and dynamic conformal arc therapy in stereotactic radiosurgery for vestibular schwannomas, Med. Dosim. 36, 62-70 (2011).

[44] J. Molinier, C. Kerr, S. Simeon, N. Ailleres, M. Charissoux, D. Azria, and P. Fenoglietto, Comparison of volumetric-modulated arc therapy and dynamic conformal arc treatment planning for cranial radiosurgery, J of App Clin Med Phys 17, 92-101 (2016).

[45] K. Otto, Volumetric modulated arc therapy: IMRT in a single gantry arc, Med. Phys. 35, $310-317$ (2008).

[46] M. Teoh, C. H. Clark, K. Wood, S. Whitaker, and A. Nisbet, Volumetric modulated arc therapy: a review of current literature and clinical use in practice, Brit. J. Radiol. 84, 967-996 (2011).

[47] D. A. Palma, W. F. A. R. Verbakel, K. Otto, and S. Senan, New developments in arc radiation therapy: a review, Cancer Treat. Rev. 36, 393-399 (2010). 
[48] T. R. Mackie, T. Holmes, S. Swerdloff, P. Reckwerdt, J. O. Deasy, J. Yang, B. Paliwal, and T. Kinsella, Tomotherapy: A new concept for the delivery of dynamic conformal radiotherapy, Med. Phys. 20, 1709-17194 (1993).

[49] R. Jeraj, T. R. Mackie, J. Balog, G. Olivera, D. Pearson, J. Kapatoes, K. Ruchala, and P. Reckwerdt, Radiation characteristics of helical tomotherapy, Med. Phys. 31, 396-404 (2004).

[50] M. A. Morales-Paliza, C. W. Coffey, and G. X. Ding, Evaluation of the dynamic conformal arc therapy in comparison to intensity-modulated radiation therapy in prostate, brain, head-and-neck and spine tumors, J of App Clin Med Phys 12, 5-19 (2011).

[51] G. C. Bentel, Radiation therapy planning, 2nd ed., McGraw-Hill, New York, 1992.

[52] O. A. Sauer, D. M. Shepard, and T. R. Mackie, Application of constrained optimization to radiotherapy planning, Med. Phys. 26, 2359-2366 (1999).

[53] J. Fiege, B. McCurdy, P. Potrebko, H. Champion, and A. Cull, Pareto: a novel evolutionary optimization approach to multiobjective IMRT planning, Med. Phys. 38, 5217-5229 (2011).

[54] T. Bortfeld, Optimized planning using physical objectives and constraints, Semin. Radiat. Oncol. 9, 20-34 (1999).

[55] S. V. Spirou and C. S. Chui, A gradient inverse planning algorithm with dosevolume constraints, Med. Phys. 25, 321-333 (1998).

[56] E. Thomas, O. Chapet, M. Kessler, T. S. Lawrence, and R. K. T. Haken, Benefit of using biological parameters (EUD and NTCP) in IMRT optimization for treatment of intrahepatic tumors, Int. J. Radiat. Oncol. Biol. Phys. 62, 571-578 (2005).

[57] J. Vaarkamp and M. Krasin, Reduction of target dose inhomogeneity in IMRT treatment planning using biologic objective functions, Int. J. Radiat. Oncol. Biol. Phys. 49, 1518-1519 (2001).

[58] P. Stavrev, D. Hristov, B. Warkentin, E. Sham, N. Stavreva, and B. G. Fallone, Inverse treatment planning by physically constrained minimization of a biological objective function, Med. Phys. 30, 2948-2958 (2003).

[59] Q. Wu, R. Mohan, A. Niemierko, and R. Schmidt-Ullrich, Optimization of intensity-modulated radiotherapy plans based on the equivalent uniform dose, Int. J. Radiat. Oncol. Biol. Phys. 52, 224-235 (2002).

[60] S. Das, T. Cullip, G. Tracton, S. Chang, L. Marks, M. Anscher, and J. Rosenman, Beam orientation selection for intensity-modulated radiation therapy based on target equivalent uniform dose maximization, Int. J. Radiat. Oncol. Biol. Phys. 55, 215-224 (2003). 
[61] S. Webb and A. E. Nahum, A model for calculating tumour control probability in radiotherapy including the effects of inhomogeneous distributions of dose and clonogenic cell density, Phys. Med. Biol. 38, 653-666 (1993).

[62] E. D. Yorke, G. J. Kutcher, A. Jackson, and C. C. Ling, Probability of radiationinduced complications in normal tissues with parallel architecture under conditions of uniform whole or partial organ irradiation, Radiother. Oncol. 26, 226-237 (1993).

[63] M. Fippel, Fast Monte Carlo dose calculation for photon beams based on the VMC electron algorithm, Med. Phys. 26, 1466-1475 (1999).

[64] E. S. M. Ali, D. J. L. Russa, and E. Vandervoort, Performance assessment of a 160-leaf beam collimation system, Med. Phys. 41, 10 (2014).

[65] B. Vanderstraeten, Accuracy of patient dose calculation for lung IMRT: a comparison of Monte Carlo, convolution/superposition, and pencil beam computations, Med. Phys. 33, 3149-3158 (2006).

[66] Monaco version 5.11 Training Guide, Elekta Business Area Software Systems, IMPAC Medical Systems, Inc., Maryland Heights, Missouri (2015).

[67] U. Jelen, M. Söhn, and M. Alber, A finite size pencil beam for IMRT dose optimization, Phys. Med. Biol. 50, 1747 - 1766 (2005).

[68] A. Ahnesjö, Collapsed cone convolution of radiant energy for photon dose calculation in heterogeneous media, Med. Phys. 16, 577 - 592 (1989).

[69] C. Lindholm, E. Cavallin-ståhl, J. Ceberg, J. Frödin, B. Littbrand, and T. R. Möller, Radiotherapy practices in Sweden compared to the scientific evidence, Acta Oncologica 42, 416-429 (2003).

[70] J. Huang, S. Zhou, P. Groome, S. Tyldesley, J. Zhang-Solomans, and W. J. Mackillop, Factors affecting the use of palliative radiotherapy in Ontario, J. Clin. Oncol. 19, 137-144 (2001).

[71] N. Thavarajah, K. Wong, L. Zhang, G. Bedard, E. Wong, M. Tsao, C. Danjoux, E. Barnes, A. Sahgal, K. Dennis, L. Holden, N. Lauzon, and E. Chow, Continued success in providing timely palliative radiation therapy at the Rapid Response Radiotherapy Program: a review of 2008-2012, Curr. Oncol. 20, e206-211 (2013).

[72] T. R. Holt and V. K. Y. Yau, Innovative program for palliative radiotherapy in Australia, J. Med. Imaging. Radiat. Oncol. 54, 76-81 (2010).

[73] D. Létourneau, R. Wong, D. Moseley, M. B. Sharpe, S. Ansell, M. Gospodarowicz, and D. A. Jaffray, Online planning and delivery technique for radiotherapy of spinal metastases using cone-beam CT: image quality and system performance, Int. J. Radiat. Oncol. Biol. Phys. 67, 1229-1237 (2007). 
[74] A. Ford, S. Bydder, and M. A. Ebert, The use of on-board imaging to plan and deliver palliative radiotherapy in a single cohesive patient appointment, J. Med. Imaging. Radiat. Oncol. 55, 633-638 (2011).

[75] R. K. S. Wong, D. Létourneau, A. Varma, J. P. Bissonnette, D. Fitzpatrick, D. Grabarz, C. Elder, M. Martin, A. Bezjak, T. Panzarella, M. Gospodarowicz, and D. A. Jaffray, A one-step cone-beam CT-enabled planning-to-treatment model for palliative radiotherapy - from development to implementation, Int. J. Radiat. Oncol. Biol. Phys. 84, 834-840 (2012).

[76] J. M. Balter, R. K. T. Haken, T. S. Lawrence, K. L. Lam, and J. M. Robertson, Uncertainties in CT-based radiation therapy treatment planning associated with patient breathing, Int. J. Radiat. Oncol. Biol. Phys. 36, 167-174 (1996).

[77] C. Park, L. Papiez, S. Zhang, M. Story, and R. D. Timmerman, Universal survival curve and single fraction dose: useful tools in understanding potency of ablative radiotherapy, Int. J. Radiat. Oncol. Biol. Phys. 70, 847-852 (2008).

[78] A. van't Riet, A. C. A. Mak, M. A. Moerland, L. H. Elders, and W. van der Zee, A conformation number to quantify the degree of conformality in brachytherapy and external beam irradiation: application to the prostate, Int. J. Radiat. Oncol. Biol. Phys. 37, 731-736 (1997).

[79] L. Feuvret, G. Noël, J.-J. Mazeron, and P. Bey, Conformity index: a review, Int. J. Radiat. Oncol. Biol. Phys. 64, 333-342 (2006).

[80] E. Shaw, C. Scott, L. Souhami, R. Dinapoli, R. Kline, J. Loeffler, and N. Farnan, Single dose radiosurgical treatment of recurrent previously irradiated primary brain tumors and brain metastases: final report of RTOG Protocol 90-05, Int. J. Radiat. Oncol. Biol. Phys. 47, 291-298 (2000).

[81] V. W. C. Wu, J. S. T. Sham, and D. L. W. Kwong, Inverse planning in threedimensional conformal and intensity-modulated radiotherapy of mid-thoracic oesophageal cancer, Brit. J. Radiol. 77, 568-572 (2004).

[82] ICRU, Report 83: Prescribing, recording, and reporting photon-beam intensitymodulated radiation therapy(IMRT), J of the ICRU 10, 1-106 (2010).

[83] I. Paddick and B. Lippitz, A simple dose gradient measurement tool to complement conformity index, J. Neurosurg. 105, 194-201 (2006).

[84] S. M. Bentzen, L. S. Constine, J. O. Deasy, A. Eisbruch, A. Jackson, L. B. Marks, R. K. T. Haken, and E. D. Yorke, Use of normal tissue complication probability models in the clinic, Int. J. Radiat. Oncol. Biol. Phys. 76, S10-S19 (2010).

[85] A. Sahgal, L. Ma, I. Gibbs, P. C. Gerszten, S. Ryu, S. Soltys, V. Weinberg, S. Wong, E. Chang, J. Fowler, and D. A. Larson, Spinal cord tolerances for stereotactic body radiotherapy, Int. J. Radiat. Oncol. Biol. Phys. 77, 548-553 (2010). 
[86] A. Sahgal, L. Ma, V. Weinberg, I. Gibbs, S. Chao, U.-K. Chang, M. Werner-Wasik, L. Angelov, E. L. Chang, M.-J. Sohn, S. G. Soltys, D. Létourneau, S. Ryu, P. C. Gerszten, J. Fowler, C. S. Wong, and D. A. Larson, Reirradiation human spinal cord tolerances for stereotactic body radiotherapy, Int. J. Radiat. Oncol. Biol. Phys. 82, 107-116 (2012).

[87] A. Sahgal, V. Weinberg, L. Ma, E. Chang, S. Chao, A. Muacevic, A. Gorgulho, S. Soltys, P. C. Gerszten, S. Ryu, L. Angelov, I. Gibbs, C. S. Wong, and D. A. Larson, Probabilities of radiation myelopathy specific to stereotactic body radiation therapy to guide safe practice, Int. J. Radiat. Oncol. Biol. Phys. 85, 341-347 (2013).

[88] J. P. Kirkpatrick, A. J. van der Kogel, and T. E. Schultheiss, Radiation dosevolume effects in the spinal cord, Int. J. Radiat. Oncol. Biol. Phys. 76, S42-S49 (2010).

[89] L. B. Marks, S. M. Bentzen, J. O. Deasy, F.-M. Kong, J. D. Bradley, I. S. Vogelius, I. el Naqa, J. L. hubbs, J. V. Lebesque, R. D. Timmerman, M. K. Martel, and A. Jackson, Radiation dose-volume effects in the lung, Int. J. Radiat. Oncol. Biol. Phys. 76, S70-S76 (2010).

[90] Y. R. Lawrence, X. A. Li, I. E. Naqa, C. A. Hahn, L. B. Marks, T. E. Merchant, and A. P. Dicker, Radiation dose-volume effects in the brain, Int. J. Radiat. Oncol. Biol. Phys. 76, S20-S27 (2010).

[91] C. Mayo, M. K. Martel, L. B. Marks, J. Flickinger, J. Nam, and J. Kirkpatrick, Radiation dose-volume effects of optic nerves and chiasm, Int. J. Radiat. Oncol. Biol. Phys. 76, S28-S35 (2010).

[92] C. Mayo, E. Yorke, and T. E. Merchant, Radiation associated brainstem injury, Int. J. Radiat. Oncol. Biol. Phys. 76, S36-S41 (2010).

[93] J. D. Ruben, S. Davis, C. Evans, P. Jones, F. Gagliardi, M. Haynes, and A. Hunter, The effect of intensity-modulated radiotherapy on radiation-induced second malignancies, Int. J. Radiat. Oncol. Biol. Phys. 70, 1530-1536 (2008).

[94] Canadian Partnership for Quality Radiotherapy, Technical quality control guidelines for medical linear accelerators and multileaf collimators, (2016).

[95] E. E. Klein, J. Hanley, J. Bayouth, F. Yin, W. Simon, S. Dresser, C. Serago, F. Aguirre, L. Ma, B. Arjomandy, C. Liu, C. Sandin, and T. Holmes, AAPM's TG-142 Protocol for quality assurance of medical accelerators, Med. Phys. 36, $4197-4212(2009)$.

[96] I. J. Das, C.-W. Cheng, R. J. Watts, A. Ahnesjö, J. Gibbons, X. A. Li, J. Lowenstein, R. K. Mitra, W. E. Simon, and T. C. Zhu, Accelerator beam data commissioning equipment and procedures: report of the TG-106 of the Therapy Physics Committee of the AAPM, Med. Phys. 35, 4187-4214 (2008). 
[97] G. Mu, E. Ludlum, and P. Xia, Impact of MLC leaf position errors on simple and complex IMRT plans for head and neck cancer, Phys. Med. Biol. 53, 77-88 (2008).

[98] K. Nithiyanantham, G. N. Mani, V. Subramani, L. Mueller, K. K. Palaniappan, and T. Kataria, Analysis of direct clinical consequences of MLC positional errors in volumetric-modulated arc therapy using 3D dosimetry system, J of App Clin Med Phys 16, 296-305 (2015).

[99] Python Software Foundation Python Language Reference, version 2.7, http://www.python.org.

[100] Y. Seppenwoolde, H. Shirato, K. Kitamura, S. Shimizu, M. van Herk, J. V. Lebesque, and K. Miyasaka, Precise and real-time measurement of 3D tumor motion in lung due to breathing and heartbeat, measure during radiotherapy, Int. J. Radiat. Oncol. Biol. Phys. 53, 822-834 (2002).

[101] H. H. Liu, P. Balter, T. Tutt, B. Choi, J. Zhang, C. Wang, M. Chi, D. Luo, T. Pan, S. Hunjan, G. Starkschall, I. Rosen, K. Prado, Z. Liao, J. Chang, R. Komaki, J. D. Cox, R. Mohan, and L. Dong, Assessing respiration-induced tumor motion and internal target volume using four-dimensional computed tomorgraphy for radiotherapy of lung cancer, Int. J. Radiat. Oncol. Biol. Phys. 68, 531-540 (2007).

[102] T. Bortfeld, S. B. Jiang, and E. Rietzel, Effects of motion on the total dose distribution, Semin. Radiat. Oncol. 14, 41-51 (2004).

[103] R. George, S. S. Vedam, T. D. Chung, V. Ramakrishnan, and P. J. Keall, The application of the sinusoidal model to lung cancer patient respiratory motion, Med. Phys. 32, 2850-2861 (2005).

[104] P. J. Keall, S. Joshi, S. S. Vedam, J. V. Siebers, V. R. Kini, and R. Mohan, Fourdimensional radiotherapy planning for DMLC-based respiratory motion tracking, Med. Phys. 32, 942-951 (2005).

[105] A. E. Lujan, E. W. Larsen, J. M. Batler, and R. K. T. Haken, A method for incorporating organ motion due to breathing into 3D dose calculations, Med. Phys. 26, 715-720 (1999).

[106] I. J. Chetty, M. Rosu, D. L. McShan, B. A. Fraass, J. M. Balter, and R. K. T. Haken, Accounting for center-of-mass target motion using convolution methods in Monte Carlo-based dose calcualtions of the lung, Med. Phys. 31, 925-932 (2004).

[107] J. Belec and B. G. Clark, Monte Carlo calculation of VMAT and helical tomotherapy dose distributions for lung stereotactic treatments with intra-fraction motion, Med. Phys. 58, 2807-2821 (2013).

[108] E. Heath, J. Unkelbach, and U. Oelfke, Incorporating uncertainties in respiratory motion into 4D treatment plan optimization, Med. Phys. 68, 3059-3071 (2009). 
[109] E. Heath and J.Seuntjens, A direct voxel tracking method for four-dimensional Monte Carlo dose calculations in deforming anatomy, Med. Phys. 33, 434-445 (2006).

[110] E. Heath, D. L. Collins, P. J. Keall, L. Dong, and J. Seuntjens, Quantification of accuracy of the automated nonlinear image matching and anatomical labeling (ANIMAL) nonlinear registration algortihm for 4D CT images of lung, Med. Phys. 34, 4409-4421 (2007).

[111] M. Serban, E. Heath, G. Stroian, D. L. Collins, and J. Seuntjens, A deformable phantom for $4 \mathrm{D}$ radiotherapy verification: design and image registration evaluation, Med. Phys. 35, 1094-1102 (2008).

[112] J. Seco, G. C. Sharp, Z. Wu, D. Gierga, F. Buettner, and H. Paganetti, Dosimetric impact of motion in free-breathing and gated lung radiotherapy: a 4D Monte Carlo study of intrafraction and interfraction effects, Med. Phys. 35, 356-366 (2008).

[113] J. Belec, Monte Carlo calculation of volumetric modulated arc therapy and helical tomotherapy dose distributions for stereotactic ablative radiotherapy lung treatments, Ph.D. thesis, Carleton University, Ottawa (2012).

[114] L. E. Court, J. Seco, X. Q. Lu, K. Ebe, C. Mayo, D. Ionascu, B. Winey, N. Giakoumakis, M. Aristophanous, R. Berbeco, J. Rottman, M. Bogdanov, D. Schofield, and T. Lingos, Use of a realistic breathing lung phantom to evaluate dose delivery errors, Med. Phys. 37, 5850-5857 (2010). 